
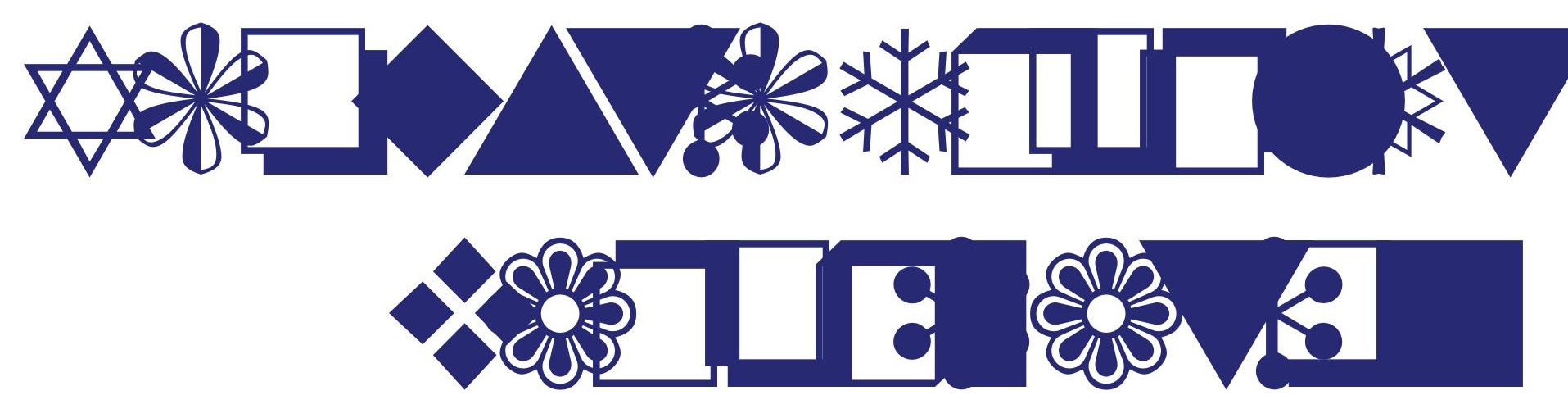

\title{
Oleksandr Shpak
}

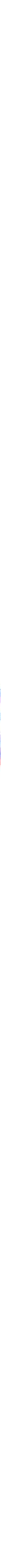


Acoustic droplet vaporization

Oleksandr Volodymyrovych Shpak 


\section{Samenstelling promotiecommissie:}

Prof. dr. ir. J.W.M. Hilgenkamp (voorzitter) Universiteit Twente, TNW

Prof. dr A.M. Versluis (promotor) Universiteit Twente, TNW

Prof. dr. ir. N. de Jong (promotor) Erasmus MC

Prof. dr. D. Lohse

Universiteit Twente, TNW

Prof. dr. ir. W. Steenbergen

Universiteit Twente, TNW

Prof. dr. ir. N. Bom

Erasmus MC

Prof. dr. C.T.W. Moonen

Dr. ir. M.D. Verweij

UMC Utrecht

TU Delft

The work in this thesis was carried out at the Physics of Fluids group of the Faculty of Science and Technology of the University of Twente.

Nederlandse titel:

Akoestische Verdamping van Druppels

Publisher:

Oleksandr Volodymyrovych Shpak, Physics of Fluids, University of Twente, P.O. Box 217, 7500 AE Enschede, The Netherlands

pof.tnw.utwente.nl

Cover illustration: Nonlinear propagation of the acoustic wave [Chapter 4 of this thesis].

Print: Gildeprint Drukkerijen B.V.

(C) Oleksandr Volodymyrovych Shpak, Enschede, The Netherlands 2014

No part of this work may be reproduced by print

photocopy or any other means without the permission

in writing from the publisher

ISBN 978-90-365-3723-0 


\title{
ACOUSTIC DROPLET VAPORIZATION
}

\author{
PROEFSCHRIFT
}

ter verkrijging van

de graad van doctor aan de Universiteit Twente,

op gezag van de rector magnificus,

prof. dr. H. Brinksma,

volgens besluit van het College voor Promoties

in het openbaar te verdedigen

op vrijdag 29 augustus 2014 om 16.45 uur

door

Oleksandr Volodymyrovych Shpak

geboren op 26 juni 1987

te Vinnytsja, Oekraine 
Dit proefschrift is goedgekeurd door de promotoren:

Prof. dr. A.M. Versluis

Prof. dr. ir. N. de Jong 


\section{Contents}

1 Introduction $\quad 1$

1.1 Ultrasound . . . . . . . . . . . . . . . . . 1

1.2 Bubbles ....................... 2

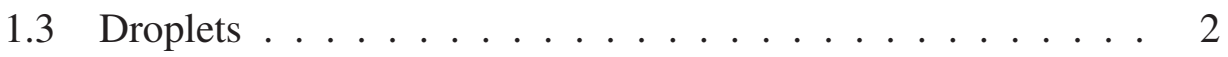

1.4 Guide through the thesis . . . . . . . . . . . 4

2 Droplets, bubble \& ultrasound interaction 9

2.1 Nonlinear propagation . . . . . . . . . . . . 10

2.1.1 Basic equations for the nonlinear ultrasound beam . . 10

2.1.2 Numerical solution for the nonlinear ultrasound beam . 11

2.1.3 Nonlinear pressure field at the focus of the beam . . . 12

2.2 Bubble dynamics . . . . . . . . . . . . . . 13

2.2.1 Dynamics of a gas bubble . . . . . . . . . 13

2.2.2 Linearization . . . . . . . . . . . . . . . 14

2.2.3 Pressure emitted by the bubble . . . . . . . . . 16

2.2.4 Secondary Bjerknes force . . . . . . . . . . . 17

2.3 Droplet dynamics . . . . . . . . . . . . . . . . . 18

2.3.1 Oscillatory translations . . . . . . . . . . 18

2.3.2 Focusing inside a spherical droplet . . . . . . . . 20

2.3.3 Radial vapor bubble expansion . . . . . . . . . . . 25

2.3.4 Activation below boiling point . . . . . . . . . 29

3 Nanodroplets 35

3.1 Introduction . . . . . . . . . . . 36

3.2 Theory . . . . . . . . . . . . . . . 38

3.2.1 Initial droplet nucleation and PFC vapor pressure . . . 38

3.2.2 Rate of bubble condensation . . . . . . . . . . 38

3.2.3 Surface tension at the vaporized droplet interface . . . 40

3.3 Experimental methods . . . . . . . . . . . . . 41 
3.3.1 Droplet preparation . . . . . . . . . . . . 41

3.3.2 Estimation of PFC droplet concentration . . . . . . . . 42

3.3.3 Droplet vaporization experiments . . . . . . . . . . 42

3.3 .4 Optical image analysis . . . . . . . . . . . . . 43

3.4 Results . . . . . . . . . . . . . . . . . . . 44

3.4.1 Droplet sizing and droplet concentration . . . . . . . . 44

3.4.2 Vaporization experiments . . . . . . . . . . . . 45

3.4.3 Post vaporization bubble size . . . . . . . . . . . . . 48

3.4 .4 Bubble survival . . . . . . . . . . . . . . . . . . . . . . . . . . 59

3.5 Discussion . . . . . . . . . . . . . . 51

3.5.1 Efficiency of droplet vaporization with applied ultrasound ...................... 51

3.5.2 Bubble behavior following vaporization . . . . . . 52

3.5.3 Bubble survival post vaporization ......... . 53

3.5.4 Limitation in resolution of initial droplet nucleation . . 56

3.6 Summary . . . . . . . . . . . . . . . . 58

4 Focusing and nucleation $\quad 65$

4.1 Introduction . . . . . . . . . . . . . . 66

4.2 Theory. . . . . . . . . . . . . . . . 68

4.2.1 Nonlinear propagation . . . . . . . . . . . 68

4.2.2 Diffraction within a spherical droplet . . . . . . . 69

4.3 Results and discussions . . . . . . . . . . . . . . . . . 71

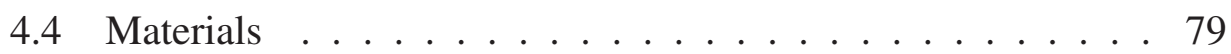

4.4 .1 Numerics . . . . . . . . . . . . . . . 79

4.4 .2 Experiments ................ 81

5 Vaporization dynamics $\quad 87$

5.1 Introduction . . . . . . . . . . . . . . . 88

5.2 Materials and methods . . . . . . . . . . . . . 90

5.2 .1 Droplets . . . . . . . . . . . . . . 90

5.2 .2 Setup ...................... 90

5.2 .3 Data analysis . . . . . . . . . . . . . . . . 92

5.3 Results . . . . . . . . . . . . . . . . . . . . 94

5.3.1 Oscillatory translations . . . . . . . . . . . . 94

5.3.2 Nucleation . . . . . . . . . . . . . . . 98

5.3.3 Vapor bubble growth . . . . . . . . . . . . 101

5.3.4 Ultrasound-driven vapor bubble growth . . . . . . 103 
5.4 Discussion . . . . . . . . . . . . . . . . 106

5.5 Conclusion . . . . . . . . . . . . 111

6 The role of gas 115

6.1 Introduction . . . . . . . . . . . . . . 115

6.2 Materials and experimental methods . . . . . . . . . 117

6.3 Model . . . . . . . . . . . . . . . . . . . 120

6.4 Results . . . . . . . . . . . . . . . . . . . 124

6.5 Summary and conclusions . . . . . . . . . . . . 132

7 Characterization of resulting bubbles 137

7.1 Introduction . . . . . . . . . . . . . . . . 138

7.2 Materials and methods . . . . . . . . . . . . . 139

7.2.1 Droplet preparations . . . . . . . . . . . . . . 139

7.2.2 Droplet vaporization . . . . . . . . . . . . . . 139

7.2.3 Vaporized droplet characterization experiment . . . . 141

7.2.4 Optical image analysis . . . . . . . . . . . . . . 141

7.3 Results . . . . . . . . . . . . . . . . . . . . 142

7.3.1 Amplitude of oscillation . . . . . . . . . . . . . 142

7.3.2 Nonlinearity of emitted pressures . . . . . . . . . 144

7.4 Discussion . . . . . . . . . . . . . . . . . . . 144

7.4.1 Bubble acoustic behaviour . . . . . . . . . . . 144

7.4.2 Suitability of vaporized PFC droplets for the role of UCAs . . . . . . . . . . . . . . . 147

7.5 Conclusion . . . . . . . . . . . . . . . . 148

8 Monodisperse droplets for ADV 153

8.1 Introduction . . . . . . . . . . . . . . . 153

8.2 Materials and methods . . . . . . . . . . . . . 154

8.2.1 Nano-microchannel chip fabrication . . . . . . . . 154

8.2.2 PFP droplet production . . . . . . . . . . . . . . . . . . . . . . . . . . . . . .

8.2.3 Droplet vaporization . . . . . . . . . . . . . . . . . . . . . . . . . . . . .

8.3 Results and discussion . . . . . . . . . . . . . . . . 159

8.3.1 Nucleation and scattering . . . . . . . . . . . . . 159

8.3.2 Symmetry of vaporization dynamics . . . . . . . . 161

8.3.3 Activation below boiling point . . . . . . . . . . . . . . . . . . . . . . . . .

8.4 Conclusion . . . . . . . . . . . . . . . . 169

9 Conclusions and Outlook 175 
Samenvatting 181

Scientific output 187

Acknowledgements 189

About the author

193 


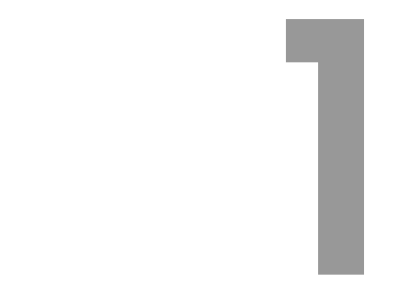

Introduction

\subsection{Ultrasound}

Medical ultrasound is widely used for imaging purposes [1]. It is an effective, mobile, inexpensive method, and has the ability to provide high resolution real time images of tissue [2]. Ultrasound imaging is performed by propagating waves through tissue and evaluating the echo which is received back. Due to the different scattering properties of the different tissues, the ultrasound receiver can evaluate the echo and construct an acoustical image.

The ultrasound wave is transmitted by an ultrasound traducer. It consists of piezoelectric crystals, which have the property of changing their volume when a voltage is applied. Applying an alternating current across piezoelectric crystals causes them to volumetrically oscillate at frequencies $(\sim \mathrm{MHz})$, causing mechanical stress on the surrounding medium, thereby converting electric energy into mechanical wave, which is then transmitted into the body. Analogously, upon receiving the echo the transducer turns the mechanical sound waves back into electrical energy, which can be measured and displayed. The transmit signal consists of a short ultrasound burst. After each burst, the electronics measures the return signal within a small window of time corresponding to the time it takes for the energy to pass through the tissue. 


\subsection{Bubbles}

Blood is a poor ultrasound scatterer and individual blood vessels are almost invisible to ultrasound. To increase the contrast of the blood pool, microbubbles can be injected into the blood stream. The microbubbles scatter ultrasound much more efficiently, allowing a very good contrast on the echo image. The contrast ability was discovered accidentally more than 40 years ago during an intravenous injection of a saline solution [3]. Saline, when injected intravenously, generates tiny microbubbles within the patient's blood vessels, thus creating an echo on the acoustical image. Since then, the second and the third generation of ultrasound contrast agents were developed. Nowadays, commercially available microbubbles are small spheres (typically $1-5 \mu \mathrm{m}$ in diameter) of gas encapsulated in a biocompatible shell. This size is similar to that of the red blood cells, which allows them to circulate inside the blood stream. The resonance frequency is directly related to the size of the bubbles (1-10 $\mu \mathrm{m}$ diameter) and coincides with the optimum imaging frequencies used in medical ultrasound imaging, 1-10 MHz.

Microbubbles are widely used also for therapy. They can enhance high intensity focused ultrasound (HIFU) therapy [4]. The bubbles increase heat uptake by the tissue and can reduce the time necessary for an ultrasound therapeutic procedure. They are sufficiently stable for time periods of approximately 15 minutes following injection [5]. Bubble oscillations and disruptions close to cells create reversible pores within the cell membrane that can enhance drug uptake [6]. Microbubbles may also be used as potential carriers to selective drug delivery [7] and for non-invasive molecular imaging [8, 9]. They can be covered with targeting ligands, such as antibodies, which bind specifically to target cells at the blood vessel wall.

\subsection{Droplets}

A novel approach is the use of liquid-based agents, rather than gas bubbles. Ultrasound can be used to phase-transition these liquid droplets into gas bubbles - a process known as acoustic droplet vaporization (ADV). Droplets are composed of a volatile perfluorocarbons (PFC), such as perfluoropentane (PFP, $29^{\circ} \mathrm{C}$ boiling point). A PFP emulsion does not spontaneously vaporize when injected in vivo at $37^{\circ} \mathrm{C}$. However, upon exposure to ultrasound above certain acoustic pressure amplitude, the PFP within the emulsion is 

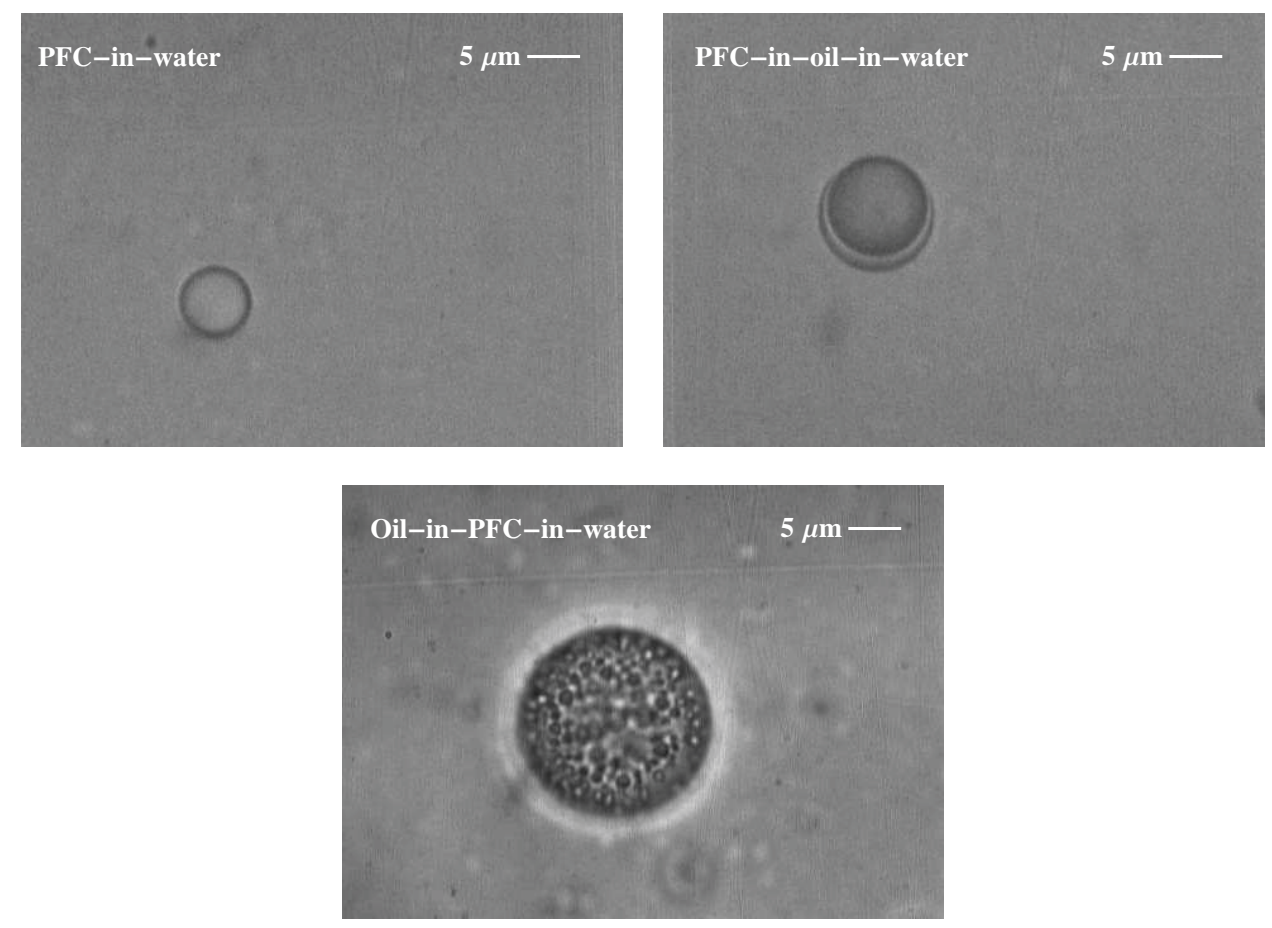

Figure 1.1: PFC-in-water, PFC-in-oil-in-water and oil-in-PFC-in-water emulsions under the microscope.

vaporized. This opens up possibilities in a wide variety of diagnostic and therapeutic applications, such as of embolotherapy [10], aberration correction [11] and drug delivery $[12,13]$. Single and double emulsions of PFC-inwater and oil-in-PFC-in-water can be prepared, for instance, to encapsulate oil soluble drugs (Fig. 1.1). PFC liquids are known in medicine, because of its biocompatibility and inertness [14]. PFC nanodroplet emulsions can be utilized for the selective extravazation in tumor regions [15]. Due to their biocompatibility and suggested ability to passively target regions of cancer growth, PFC droplets represent an attractive tool for cancer diagnosis. PFC droplets may also extravasate and get retained in the extravascular space due to the enhanced permeability and retention effect in tumors [16, 17]. Extravasated droplets may be acoustically converted into gas bubbles allowing for ultrasound tumor imaging. At the same time PFC droplets are rich in fluorine, which makes them potential candidates as a contrast agent for the MRI 
imaging. The availability of both intravascular contrast agents (microbubbles), and tumor-specific extravascular contrast agents (nanodroplets), would significantly increase diagnostic and therapeutic capabilities. Moreover, the droplets may be used to deliver chemotherapeutic agents to tumor regions, and locally release them upon exposure to triggered ultrasound [18].

\subsection{Guide through the thesis}

From this introduction it is evident that a full understanding of the physical mechanisms underlying ADV is essential to provide input for the clinical aspects of this novel approach. The questions of ADV are, what actually triggers the vaporization, why is the required pressure amplitude to initiate the vaporization inversely proportional to its frequency and droplet size, how fast is the vaporization process, what is the role of dissolved gas in phase conversion process, how stable are the resulting bubbles, what are the characteristics of vaporization of submicron droplets, how to produce and what are the benefits of employing monodisperse droplets.

In this thesis we have studied all the above mentioned aspects of ADV. Once the vaporization process is initiated, the growing vapor bubble/droplet system strongly interacts with the applied acoustic wave. Thus, it is important to know the physics of bubble and droplet interaction with ultrasound. In Chapter 2 we give the basics of the dynamics of gas bubbles forced by ultrasound, linearization of the Rayleigh-Plesset equation, the pressures reradiated by the bubble, and Bjerknes forces. We also introduce the interaction of droplets with ultrasound, such as oscillatory translations, focusing within the droplet sphere, and radial bubble expansion due to the phase change and gas diffusion.

In Chapter 3 we conduct an initial ultra-high-speed optical imaging study to examine the vaporization of submicron droplets and observe the newly created microbubbles in the first microseconds after vaporization. As a result of this study we show that additional factors, such as coalescence and bubble shell properties, are important and should be carefully considered for the production of microbubbles for use in medical imaging.

In Chapter 4 we explain the physics of the initiation of acoustic droplet vaporization. We present the mechanism which explains the hitherto counterintuitive dependence of the nucleation threshold on the ultrasound frequency. We show that ADV is initiated by a combination of two phenomena: highly 
nonlinear distortion of the acoustic wave before it hits the droplet, and focusing of the distorted wave by the droplet itself. At high excitation pressures, nonlinear distortion causes significant superharmonics with wavelengths of the order of the droplet size.

In Chapter 5 we underline and explain three distinct regimes of ADV: 1. prior to nucleation, a regime of droplet deformation and oscillatory translations within the surrounding fluid; 2 . a regime characterized by the rapid growth of a vapor bubble enhanced by ultrasound-driven rectified heat transfer; and 3. a final phase characterized by a relatively slow expansion, after ultrasound stops, that is fully dominated by heat transfer. We propose a method to measure the moment of inception of the nucleation event with respect to the phase of the ultrasound wave. We implement a simple physical model captures quantitatively all of the features of the subsequent vapor bubble growth.

In Chapter 6 we model the vapor-gas bubble dynamics, based on a RayleighPlesset-type equation, including thermal and gas diffusion inside the liquid. We underline the fundamental role of gas diffusion in order to prevent total recondensation of the bubble at collapse during the first peak-positive acoustic pressure half-cycle.

In Chapter 7 we examine the acoustic characteristics of microbubbles created from vaporized submicron perfluorocarbon droplets with a fluorosurfactant coating. We observe the acoustic response of individual microbubbles to low intensity diagnostic ultrasound on clinically relevant timescales of hundreds of milliseconds after vaporization. We show that the vaporized droplets oscillate nonlinearly, and exhibit a resonant bubble size shift and increased damping relative to uncoated gas bubbles due to the presence of coating material. The results of Chapter 7 suggest that vaporized submicron PFC droplets have the acoustic characteristics necessary for their potential use as ultrasound contrast agents in clinical practice.

In Chapter 8 we fabricate glass chips with a micro-nanochannel step geometry, which allow us to produce monodisperse submicron droplets as small as $260 \mathrm{~nm}$ in radius. We investigate the nucleation and growth of monodisperse droplets at nanoseconds time scale. We show that the vaporization of monodisperse droplets have a high degree of symmetry and that the activation threshold error bars are much smaller compared to polydisperse droplets. In addition, we investigate the activation of monodisperse droplets in ambient temperature below bolling point.

Finally, the conclusions and outlook are presented in Chapter 9. 


\section{References}

[1] T. L. Szabo, Diagnostic Ultrasound Imaging (Inside Out. Academic Press) (2004).

[2] K. K. Shung, Diagnostic Ultrasound (Imaging and Blood Flow Measurements, CRC) (2006).

[3] R. Gramiak and P. M. Shah, "Echocardiography of the aortic root", Investigative Radiology 3, 356-366 (1968).

[4] E. C. Unger, T. Porter, W. Culp, R. Labell, T. Matsunaga, and R. Zutshi, "Therapeutic applications of lipid-coated microbubbles", Advanced Drug Delivery Reviews 59, 1291-1314 (2004).

[5] A. L. Klibanov, "Microbubble contrast agents: targeted ultrasound imaging and ultrasound-assisted drug-delivery applications", Investigative Radiology 41, 354-362 (2006).

[6] R. Karshafian, P. D. Bevan, R. Williams, S. Samac, and P. N. Burns, "Sonoporation by ultrasound-activated microbubble contrast agents: effect of acoustic exposure parameters on cell membrane permeability and cell viability", Ultrasound Med. Biol. 35, 847-860 (2009).

[7] E. C. Unger, E. Hersh, M. Vannan, T. O. Matsunaga, and T. McCreery, "Local drug and gene delivery through microbubbles", Progress in Cardiovascular Diseases 41, 45-54 (2009).

[8] J. R. Lindner, "Microbubbles in medical imaging: current applications and future directions", Nat. Rev. Drug Discov. 35, 527-533 (2004).

[9] A. L. Klibanov, "Microbubble contrast agents: Targeted ultrasound imaging and ultrasound-assisted drug-delivery applications", Invest. Radiol. 41, 354-362 (2006).

[10] M. Zhang, M. L. Fabiilli, K. J. Haworth, J. B. Fowlkes, O. D. Kripfgans, W. W. Roberts, K. A. Ives, and P. L. Carson, "Sonoporation by ultrasound-activated microbubble contrast agents: effect of acoustic exposure parameters on cell membrane permeability and cell viability", Ultrasound Med. Biol. 36, 1691-1703 (2010). 
[11] C. M. Carneal, O. D. Kripfgans, J. Krucker, P. L. Carson, and J. B. Fowlkes, "A tissue-mimicking ultrasound test object using droplet vaporization to create point targets", Pharmaceutical Research 58, 20132025 (2011).

[12] M. L. Fabiilli, J. A. Lee, O. D. Kripfgans, P. L. Carson, and J. B. Fowlkes, "Delivery of water-soluble drugs using acoustically triggered per uorocarbon double emulsions", Ultrasound Med. Biol. 27, 27532765 (2010).

[13] M. L. Fabiilli, K. J. Haworth, I. E. Sebastian, O. D. Kripfgans, P. L. Carson, and J. B. Fowlkes, "Delivery of chlorambucil using an acousticallytriggered perfluoropentane emulsion”, Ultrasound Med. Biol. 36, 13641375 (2010).

[14] G. P. Biro, P. Blais, and A. L. Rosen, "Peruorocarbon blood substitutes", CRC Critical Reviews in Oncology/Hematology 6, 311-374 (1987).

[15] D. M. Long, F. K. Multer, A. G. Greenburg, G. W. Peskin, E. C. Lasser, W. G. Wickham, and C. M. Sharts, "Tumor imaging with x-rays using macrophage uptake of radiopaque fluorocarbon emulsions", Surgery 84, 104-112 (1978).

[16] N. Y. Rapoport, Z. Gao, and A. Kennedy, "Multifunctional nanoparticles for combining ultrasonic tumor imaging and targeted chemotherapy", Journal of the National Cancer Institute 99, 1095-1106 (2007).

[17] P. Zhang and T. Porter, "An in vitro study of a phase-shift nanoemulsion: a potential nucleation agent for bubble-enhanced hifu tumor ablation", Ultrasound Med. Biol. 36, 1856-1866 (2010).

[18] N. Y. Rapoport, A. M. Kennedy, J. E. Shea, C. L. Scaife, and K.-H. Nam, "Controlled and targeted tumor chemotherapy by ultrasound-activated nanoemulsions/microbubbles", Journal of Controlled Release 138, 268276 (2009). 


\section{2 Droplets, bubble \& ultrasound interaction}

The interaction of droplets and bubbles with ultrasound has been studied extensively in the last 25 years. Microbubbles are broadly used in diagnostic and therapeutic medical applications, for instance, as ultrasound contrast agents. They have a similar size as red blood cells, and thus are able to circulate within the blood vessels. Perfluorocarbon liquid droplets can be a potential new generation of microbubble agents, as they can be triggered with ultrasound converting them into gas bubbles. Prior to activation, they are at least 5 times smaller in diameter than the resulting bubbles. Together with the violent nature of the phase-transition the droplets can be used for local drug delivery, embolotherapy, HIFU enhancement and for tumor imaging. Here we explain the basics of the bubble dynamics, described with the RayleighPlesset equation, its resonance frequency, damping and quality factor. We show the elegant calculation of the above characteristics in case of small amplitude oscillations by linearizing the equations. The effect and importance of a bubble coating and effective surface tension are discussed. We give the main

\footnotetext{
${ }^{\ddagger}$ Submitted as a book chapter: Oleksandr Shpak, Martin Verweij, Nico de Jong, and Michel Versluis, "Droplets, Bubble \& Ultrasound interaction", "Therapeutic Ultrasound" book to Springer's Editions, Editor: Jean-Michel Escoffre.
} 
characteristics of the power spectrum of bubble oscillations. Preceding bubble dynamics the ultrasound propagation is introduced. We explain the speed of sound, the nonlinearity and attenuation terms. We discuss the bubble ultrasound scattering and how it depends on the wave-shape of the incident wave. Finally we introduce the droplets interaction with ultrasound. We explain the ultrasound focusing concept within a droplets sphere, droplet shaking due to media compressibility, and droplet phase-conversion dynamics.

\subsection{Nonlinear propagation}

The amplitude of the acoustic pressure that is required to nucleate droplets in ADV turns out to be very high [1]. To obtain a sufficiently high pressure, a focused ultrasound transducer is applied and the droplet is placed in the focal area of the emitted beam. Moreover, the frequency of the emitted ultrasound wave is several MHz. In a typical ADV experiment, the ultrasound wave travels a few centimeters [1-7] before impinging on the droplet. The high pressure, high frequency, applied focusing, and long propagation distance are all factors that strengthen the nonlinear behavior of the ultrasound wave $[8,9]$. As a result, the wave that impinges on the droplet will be a highly deformed version of the one that is emitted by the transducer. This has important consequences for the focusing inside the droplet, as will be demonstrated in Subsection 2.3.2.

\subsubsection{Basic equations for the nonlinear ultrasound beam}

Similar to most cases involving nonlinear medical ultrasound, the description of the beam that hits the droplet can be based on the Westervelt equation [10, 11]:

$$
\nabla^{2} p-\frac{1}{c_{0}} \frac{\partial^{2} p}{\partial t^{2}}+\frac{\delta}{c_{0}^{4}} \frac{\partial^{3} p}{\partial t^{3}}=-\frac{\beta}{\rho_{0} c_{0}^{4}} \frac{\partial^{2} p^{2}}{\partial t^{2}},
$$

where $\nabla^{2}=\partial^{2} / \partial x^{2}+\partial^{2} / \partial y^{2}+\partial^{2} / \partial z^{2}$ is the Laplace operator and $p=$ $p(x, y, z, t)$ denotes the acoustic pressure. The medium in which the ultrasound wave propagates is characterized by the ambient speed of sound $c_{0}$, the ambient density of mass $\rho_{0}$, the diffusivity of sound $\delta$, and the coefficient of nonlinearity $\beta$. Unfortunately, closed-form analytical solutions of this equation do not exist and its numerical solution generally requires considerable computational effort. However, in the present case of a narrow, fo- 
cused beam and a homogeneous medium, some simplifying assumptions can be made. First, it may be assumed that the predominant direction of propagation is along the transducer axis, which is taken in the $z$-direction. In this case we can replace the ordinary time coordinate $t$ by the retarded time coordinate $\tau=\left(t-t_{0}\right)-\left(z-z_{0}\right) / c_{0}$, which keeps the same value when traveling along with the wave. Here, $t_{0}$ is the time at which the transducer emits the pressure wave, and $z_{0}$ is the axial position of the transducer. The equivalent of Eq. 2.1 in the co-moving time frame is:

$$
\nabla^{2} \bar{p}-\frac{2}{c_{0}} \frac{\partial^{2} \bar{p}}{\partial z \partial \tau}+\frac{\delta}{c_{0}^{3}} \frac{\partial^{4} \bar{p}}{\partial \tau^{3}}=-\frac{\beta}{\rho_{0} c_{0}^{4}} \frac{\partial^{2} \bar{p}^{2}}{\partial \tau^{2}},
$$

with $\bar{p}=\bar{p}(x, y, x, \tau)$ denoting the acoustic pressure in the co-moving time frame. Second, it may be assumed that in the retarded time frame the axial derivative $\partial^{2} \bar{p} / \partial z^{2}$ is much smaller than the lateral derivatives $\partial^{2} \hat{p} / \partial x^{2}$ and $\partial^{2} \hat{p} / \partial y^{2}$. This motivates the use of the parabolic approximation $\nabla^{2} \bar{p} \approx \nabla_{\perp}^{2} \bar{p}$, where $\nabla_{\perp}^{2}=\partial^{2} / \partial x^{2}+\partial^{2} / \partial y^{2}$ is the Laplace operator in the lateral plane. This approximation is valid for waves propagating under at most 20 degrees of the transducer axis [12]. Applying the parabolic approximation to Eq. 2.2 and rearranging terms results in the Khokhlov-Zabolotskaya-Kuznetsov (KZK) equation $[13,14]$ :

$$
\frac{\partial^{2} \bar{p}}{\partial z \partial \tau}=\frac{c_{0}}{2} \nabla_{\perp}^{2} \bar{p}+\frac{\delta}{2 c_{0}^{3}} \frac{\partial^{3} \bar{p}}{\partial \tau^{3}}+\frac{\beta}{2 \rho_{0} c_{0}^{3}} \frac{\partial^{2} \bar{p}^{2}}{\partial \tau^{2}} .
$$

Dedicated coordinate transformations may be applied to improve the numerical solution in the far field $[15,16]$ or to adapt to specific forms of focused beams [17], but these will not be discussed here.

\subsubsection{Numerical solution for the nonlinear ultrasound beam}

We will follow a well-known numerical solution strategy $[18,19]$ that is based on the time-integrated version of Eq. 2.3:

$$
\frac{\partial \bar{p}}{\partial z}=\frac{c_{0}}{2} \int_{-\infty}^{\tau} \nabla_{\perp}^{2} \bar{p}\left(\tau^{\prime}\right) d \tau^{\prime}+\frac{\delta}{2 c_{0}^{3}} \frac{\partial^{2} \bar{p}}{\partial \tau^{2}}+\frac{\beta \bar{p}}{\rho_{0} c_{0}^{3}} \frac{\partial \bar{p}}{\partial \tau} .
$$

The first term at the right-hand side of this equation accounts for the diffraction of the beam, the second term for its attenuation, and the third term for its 
nonlinear distortion. Further, the solution strategy is based on the split-step approach. This means that the field $\bar{p}$ is stepped forward over a succession of parallel planes with mutual distance $\Delta z$, where the field $\bar{p}\left(x, y, z_{0}, \tau\right)$ in the transducer plane acts as the starting plane. The stepsize $\Delta z$ is taken sufficiently small, which allows that each of the above phenomena may be accounted for in separate substeps [20]. Therefore, the total step $z \rightarrow z+\Delta z$ involves the numerical solution of the separate equations:

$$
\begin{aligned}
\frac{\partial \bar{p}}{\partial z} & =\frac{c_{0}}{2} \int_{-\infty}^{\tau} \nabla_{\perp}^{2} \bar{p}\left(\tau^{\prime}\right) d \tau^{\prime}, \\
\frac{\partial \bar{p}}{\partial z} & =\frac{\delta}{2 c_{0}^{3}} \frac{\partial^{2} \bar{p}}{\partial \tau^{2}}, \\
\frac{\partial \bar{p}}{\partial z} & =\frac{\beta \bar{p}}{\rho_{0} c_{0}^{3}} \frac{\partial \bar{p}}{\partial \tau},
\end{aligned}
$$

over the same interval, where the result of solving one equation is used as the input for solving the next one. A numerical implementation of the above process is used to step the acoustic pressure from the transducer to the focus of the beam, i.e. the location of the droplet. For convenience is now assumed that the droplet is located at the origin of the coordinate system and $t_{0}=$ $z_{0} / c_{0}$, which makes that at droplet $\tau=t$. For ease of notation the bar and the coordinates of the droplet will be suppressed, and the pressure at the droplet position, as obtained from the numerical solution of the KZK-equation, will simply be indicated by $p_{K Z K}(t)$.

\subsubsection{Nonlinear pressure field at the focus of the beam}

The nonlinear pressure field at the focus of the beam can be expanded in a Fourier series:

$$
p_{K Z K}(t)=\sum_{n=0}^{\infty} a_{n} \cos \left(n \omega t+\phi_{n}\right)=\operatorname{Re}\left[\sum_{n=0}^{\infty} a_{n} e^{i\left(n \omega t+\phi_{n}\right)}\right],
$$

where $a_{n}$ and $\phi_{n}$ are the amplitudes and the phases of the $n$-th harmonic component of the ultrasound wave. For convenience, all the subsequent derivations will be given in the complex representation, so we will omit taking the real part and simply write:

$$
p_{K Z K}(t)=\sum_{n=0}^{\infty} a_{n} e^{i\left(n \omega t+\phi_{n}\right)} .
$$


Because nonlinear deformation of the waveform builds up over distance and the droplet is four orders of magnitude smaller in size than the distance to the transducer, the additional nonlinear distortion inside the droplet is neglected. This implies that wave propagation inside the droplet is considered linear, so the superposition theorem holds and the focusing of each harmonic component in the droplet may be analyzed on an individual basis, as will be done in Subsection 2.3.2.

\subsection{Bubble dynamics}

\subsubsection{Dynamics of a gas bubble}

Bubble radial oscillations are governed by the Rayleigh-Plesset equation:

$$
\ddot{R} R+\frac{3}{2} \dot{R}^{2}=\frac{\Delta P}{\rho},
$$

where $R, \dot{R}$, and $\ddot{R}$ are the radius, the velocity and the acceleration of the bubble wall, respectively, and $\rho$ is the density of the liquid. $\Delta P=P_{L}(R)-$ $P_{\infty}$ is the pressure difference between the liquid at the bubble wall $P_{L}(R)$ and the external pressure infinitely far from the bubble $p_{\infty}$. Eq. 2.10 was first described by Lord Rayleigh [21] for the case $\Delta \mathrm{P}=0$ and was later refined [22-25]. It is derived for a spherically symmetric bubble and follows from the Bernoulli's equation and the continuity equation [26]. Eq. 2.10 assumes spherical symmetry of the bubble, and the motion of the liquid around the bubbles is considered to be spherically symmetric. The liquid is incompressible.

The bubble is assumed to be much smaller than the acoustic wavelength, such that acoustic pressure is considered to be uniform. Thus, the pressure at infinity is the sum of the acoustic forcing $P(t)$ and the ambient pressure $P_{0}$ :

$$
\mathrm{p}_{\infty}=\mathrm{P}(\mathrm{t})+\mathrm{P}_{0}
$$

The interfacial pressure acting on the liquid at the bubble wall consists of the Laplace pressure $2 \sigma / R_{0}$, viscous pressure $4 \mu \dot{R} / R$ and the gas pressure $P_{g}$. Neglecting the vapor pressure of the liquid, the gas pressure inside the bubble as a function of the bubble radius $R$ can be described by the ideal gas relation $\mathrm{P}_{\mathrm{g}} \mathrm{V}^{\gamma}=$ const, where $\gamma$ is the polytropic constant and $\mathrm{V} \propto \mathrm{R}^{3}$ is the bubble volume. For this derivation we first neglect the gas diffusion. Thus, the total 
number of gas molecules inside the bubble is constant. In equilibrium the pressure inside the bubble $P_{e q}$ is equal to the sum of the ambient pressure $P_{0}$ and the Laplace pressure:

$$
P_{e q}=P_{0}+\frac{2 \sigma}{R_{0}}
$$

where $\sigma$ and $R_{0}$ are the surface tension and the equilibrium radius, respectively. In a combination with the ideal gas law, the dependence of the gas pressure as a function of the bubble radius can be written as $P_{g}=\left(P_{0}+\frac{2 \sigma}{R_{0}}\right)\left(\frac{R_{0}}{R}\right)^{3 \gamma}$. The right-hand side of Eq. 2.10 can be then written as:

$$
\Delta \mathrm{P}=\left(\mathrm{P}_{0}+\frac{2 \sigma}{\mathrm{R}_{0}}\right)\left(\frac{\mathrm{R}_{0}}{\mathrm{R}}\right)^{3 \gamma}-\mathrm{P}_{0}-\frac{2 \sigma}{\mathrm{R}}-4 \mu \frac{\dot{\mathrm{R}}}{\mathrm{R}}-\mathrm{P}(\mathrm{t}),
$$

which gives the final form of bubble dynamic equation:

$$
\rho\left(\ddot{R} R+\frac{3}{2} \dot{R}^{2}\right)=\left(P_{0}+\frac{2 \sigma}{R_{0}}\right)\left(\frac{R_{0}}{R}\right)^{3 \gamma}-P_{0}-\frac{2 \sigma}{R}-4 \mu \frac{\dot{R}}{R}-P(t) .
$$

The microbubbles in the ultrasound contrast agents can be encapsulated with a phospholipid, protein or polymer coating, preventing bubbles from dissolution. For more details please see refs. [27-29]. The viscoelastic coating also contributes to an increased stiffness and to additional viscous damping [30].

\subsubsection{Linearization}

The acoustic pressure typically has the form of a sinusoidal oscillation $\mathrm{P}(\mathrm{t})=$ $P_{A} \sin (\omega t)$, with $P_{A}$ being the driving pressure amplitude, and $\omega$ the driving pressure angular frequency. With relatively small oscillation amplitudes Eq. 2.10 can be linearized. To rewrite Eq. 2.10 in linear terms we express the bubble radius $R$ as:

$$
\mathrm{R}=\mathrm{R}_{0}(1+x),
$$

with $R_{0}$ the equilibrium radius, as before, and $x \ll 1$ is small dimensionless perturbation to the radius. Substituting Eq. 2.15 into Eq. 2.10 and retaining only first-order terms, $x, \dot{x}$, and $\ddot{x}$, gives: 


$$
\ddot{x}+2 \beta \dot{x}+\omega_{0}^{2} x=\frac{P_{A}}{\rho R_{0}^{2}} \sin (\omega t),
$$

where

$$
\omega_{0}=\sqrt{\frac{1}{\rho R_{0}^{2}}\left[3 \gamma\left(P_{0}+\frac{2 \sigma}{R_{0}}\right)-\frac{2 \sigma}{R_{0}}\right]}
$$

the eigenfrequency of bubble oscillations, and:

$$
\beta=\frac{2 \mu}{\rho R_{0}^{2}},
$$

the damping due to viscosity. The damping has the dimensions of the reversed time $\left[\mathrm{s}^{-1}\right]$ and represents how fast the amplitude of oscillations is decaying in time due to the energy loss.

The solution to the equation Eq. 2.16 is:

$$
x(t)=X_{t} e^{-\beta t} \cos \left(\omega_{1} t\right)+X_{s} \cos \left(\omega t+\phi_{1}\right),
$$

with the $\phi_{1}$ is the phase shift between the two terms:

$$
\phi_{1}=\arctan \left(\frac{\omega_{0}^{2}-\omega^{2}}{2 \beta \omega}\right)
$$

The first term of Eq. 2.19 is the transient solution. Its amplitude dampens out in time as $X_{t} e^{-\beta t}$, where $X_{t}$ is the amplitude of transient oscillations at time $t_{0}=0$. Not only the viscosity of water can contribute to the damping, but also the acoustic reradiation and the viscosity of the coating shell and thermal damping. For more details please see [31]. The frequency of the transient solution is equal to $\omega_{1}=\sqrt{\omega_{0}^{2}-\beta^{2}}$. The amplitude of the transient solution $X_{t}$ depends strongly on the initial conditions.

The second term of Eq. 2.19 is the steady-state solution. The amplitude of the steady-state response depends on the driving frequency as:

$$
X_{s}=\frac{P_{A}}{\rho R_{0}^{2}} \frac{1}{\sqrt{\left(\omega_{0}^{2}-\omega^{2}\right)^{2}+4 \omega^{2} \beta^{2}}}
$$

The resonance frequency $\omega_{\text {res }}$ of the system, by definition, corresponds to the maximal amplitude of the steady-state solution. $X_{s}$ is at maximum, when 
the denominator in the Eq 2.21 is at minimum. Thus, the resonance frequency relates to the eigenfrequency $\omega_{0}$ as:

$$
\omega_{\text {res }}=\sqrt{\omega_{0}^{2}-2 \beta^{2}}
$$

The smaller the damping $\beta$ the closer the resonance frequency to the eigenfrequency of the bubble oscillations. Additionally, for large bubbles, when the Laplace pressure is small compared to the ambient pressure Eq. 2.17 simplifies to:

$$
\omega_{M}=2 \pi f_{M}=\sqrt{3 \gamma \frac{P_{0}}{\rho R_{0}^{2}}},
$$

with $f_{M}$ the Minnaert eigenfrequency, resonance frequency of the bubble [32]. Relation Eq. 2.23 tells us, that the resonance frequency can be estimated directly from the bubble radius $R_{0}$. For a bubble in water at standard pressure $P_{0}=100 \mathrm{kPa}, \rho=1000 \mathrm{~kg} / \mathrm{m}^{3}$ ), the equation becomes $f_{M} R_{0} \approx$ $3.26 \mu \mathrm{m} \cdot \mathrm{MHz}$. The smaller the bubble radius, the higher the resonance frequency becomes.

It is insightful to make the analogy to the classical mass-spring system. The dynamics of the classical mass spring system is governed by the equation:

$$
\ddot{x^{\prime}}+2 \frac{\beta^{\prime}}{\mathrm{m}} \dot{x}+\omega_{0}^{\prime 2} x=\frac{\mathrm{F}_{0}}{\mathrm{~m}} \sin (\omega t),
$$

where $\omega_{0}^{\prime}=\sqrt{\mathrm{k} / \mathrm{m}}$ is the eigenfrequency, $\beta^{\prime}$ is the damping constant, $k$ is the spring stiffness, $F_{0}$ is the driving force, and $m$ is the mass. Eq. 2.24 has the same form as Eq. 2.16. Thus a gas inside the bubble, represented by the polytropic constant $\gamma$, acts as the restoring force, the liquid around the bubble acts as a mass $\left(4 \pi R_{0}^{3} \rho\right)$, and ultrasound is acting as a driving force $\left(12 \pi \gamma \mathrm{R}_{0} \mathrm{P}_{0}\right)$.

\subsubsection{Pressure emitted by the bubble}

Far from the bubble wall, at a distance $r$, the velocity of the liquid $v_{r}$ can be calculated from the continuity equation [33]:

$$
4 \pi r^{2} v_{r}=4 \pi R^{2} \dot{R}
$$




$$
v_{r}=\frac{R^{2}}{r^{2}} \dot{R}
$$

The liquid is incompressible and the bubble wall and the liquid motion around the bubble are spherically symmetric. $\dot{R}$ is the velocity of the bubble wall in the radial direction, as before.

The pressure field, generated by the radial bubble wall oscillations, can be calculated from the Euler equation [33]:

$$
\rho \frac{\partial v}{\partial t}+\frac{\partial p}{\partial r}=0
$$

where $p$ is the pressure emitted by the bubble. In Eq. 2.27 we omit the nonlinear convective term. Substituting the expression of the velocity field (Eq. 2.26) into Eq. 2.27 gives the pressure gradient:

$$
\frac{\partial p}{\partial r}=-\frac{\rho}{r^{2}} \frac{d}{d t}\left(R^{2} \dot{R}\right),
$$

and the pressure emitted by the bubble:

$$
p=\frac{\rho}{r} \frac{d}{d t}\left(R^{2} \dot{R}\right)=\rho\left(\frac{R^{2} \ddot{R}+2 R \dot{R}^{2}}{r}\right)
$$

\subsubsection{Secondary Bjerknes force}

Let us now consider two interacting gas bubbles, separated by a distance $l$. The distance between the bubbles 1 and 2 is much larger that their radii $R_{1}(t)$ and $R_{2}(t)$. Thus, we can consider the motion of the liquid around the bubbles to be spherically symmetric. Bubble 2 with volume $V_{2}=\frac{4}{3} \pi R_{2}^{3}$ experiences a force $F_{12}$ as a result of the pressure emitted by bubble $1, P_{1}$ [26]:

$$
\mathrm{F}_{12}=-\mathrm{V}_{2} \nabla \mathrm{p}_{1}
$$

The force is directed along the line, which connects the centers of the two interacting bubbles. Substitution of Eq. 2.28 (expression for the pressure gradient generated by the first bubble) into Eq. 2.30 yields the force of the first bubble on the second one at a distance $l$ :

$$
F_{12}=-\left.V_{2} \frac{\partial p_{1}}{\partial r}\right|_{r=l}=V_{2} \frac{\rho}{l^{2}} \frac{d}{d t}\left(R_{1}^{2} \dot{R}_{1}\right)=\frac{\rho}{4 \pi l^{2}} V_{2} \frac{d^{2} V_{1}}{d t^{2}}
$$


where $V_{1}=\frac{4}{3} \pi R_{1}^{3}$ the volume of bubble 1 .

The net radiation force acting on a neighboring bubble is called the secondary Bjerknes force $F_{B}$ after Bjerknes [34]. The time averaged equation $\left\langle F_{12}\right\rangle$ is obtained by integrating Eq. 2.31 over the period of volume oscillations by partial integration:

$$
\mathrm{F}_{\mathrm{B}}=\left\langle\mathrm{F}_{12}\right\rangle=-\frac{\rho}{4 \pi \mathrm{d}^{2}}\left\langle\dot{\mathrm{V}}_{1} \dot{\mathrm{V}}_{2}\right\rangle
$$

A positive value of $\left\langle\dot{V}_{1} \dot{V}_{2}\right\rangle$ corresponds to attraction of the bubbles, and a negative value to repulsion. This means that the bubbles that oscillate with the same phase will attract each other. Note also the symmetry of Eq. 2.32. To calculate the force of the second bubble on the first one $\left\langle F_{21}\right\rangle$, one just need to exchange indexes $1 \leftrightarrow 2$. $\left\langle\mathrm{F}_{21}\right\rangle$ has the same magnitude but an opposite direction as $\left\langle\mathrm{F}_{12}\right\rangle$.

\subsection{Droplet dynamics}

\subsubsection{Oscillatory translations}

The typical pressure amplitudes which are used to activate perfluorocarbon droplets are two orders of magnitude higher that those used to drive ultrasound contrast agents. Water itself always experiences periodic compression as a result of ultrasound forcing [26]. Let us express such oscillations as:

$$
\epsilon=\epsilon_{0} \sin (\omega t-k x),
$$

where $\epsilon$ is the fluid particle displacement. The acoustic impedance, by definition, is the ratio of the driving pressure to the fluid particle velocity [35]:

$$
\mathrm{Z}=\mathrm{P}_{\mathrm{A}} / \dot{\mathrm{\epsilon}}_{0},
$$

where $\dot{\epsilon}_{0}$ is the particle displacement velocity amplitude, and $\mathrm{P}_{\mathrm{A}}$ is the acoustic pressure amplitude. The $\dot{\epsilon}_{0}$ particle velocity amplitude relates to the particle displacement amplitude $\epsilon_{0}$ as $\dot{\epsilon}_{0}=\omega \epsilon_{0}$, which follows from Eq. 2.33 by taking its time derivative. The $\mathrm{dP}$ pressure change with respect to the equilibrium value is related to a $d V$ volume change by the bulk modulus $B$, defined by [26]:

$$
d P=-B \frac{d V}{V}
$$




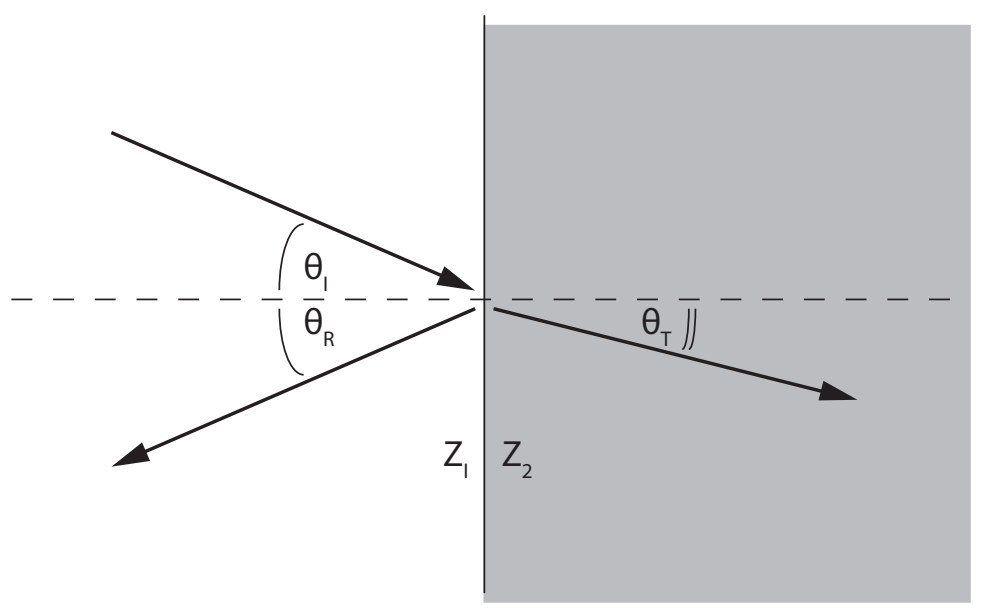

Figure 2.1: Schematics of reflection and transmission of a wave of displacement.

Eq. 2.35 can be used to calculate the acoustic pressure $P$ at any given spatial point $x_{0}$ as $P\left(x_{0}\right)=-\left.B \frac{\partial \epsilon}{\partial x}\right|_{x=x_{0}}$. Applying this relation to Eq. 2.33 gives $P_{A}=B k \epsilon_{0}$, or $P_{A}=B \frac{k}{\omega} \dot{\epsilon}_{0}$. The acoustic impedance Eq. 2.34 can then be written as:

$$
\mathrm{Z}=\mathrm{B} \frac{\mathrm{k}}{\omega}
$$

or by using the equation for the wave speed $c=\omega / k=\sqrt{B / \rho}$ :

$$
\mathrm{Z}=\rho \mathrm{c},
$$

With the relations given above, one can now estimate the oscillatory translational amplitude. For the case of $f=3.5 \mathrm{MHz}, \mathrm{P}_{\mathrm{A}}=8 \mathrm{MPa}$, and $\mathrm{c}_{w}$ $=1522 \mathrm{~m} / \mathrm{s}$ the speed of sound in water at $37^{\circ} \mathrm{C}$, the amplitude is $\epsilon_{0}=$ $\mathrm{P}_{\mathrm{A}} / 2 \pi \rho \mathrm{fc}_{w}=210 \mathrm{~nm}$.

The acoustic impedance $Z=\rho c$ has the analogy with a refractive index $n$ in optics. The ultrasound wave at the interface of two substances with different acoustic impedances $Z_{1}$ and $Z_{2}$ will experience a reflection and a refraction, similarly as light would experience at the interface with two different refractive indexes $n_{1}$ and $n_{2}$.

Let us now denote $\theta_{\mathrm{I}}, \theta_{\mathrm{R}}$, and $\theta_{\mathrm{T}}$ the incident, the translated and the reflected angles, respectively (see Fig. 2.1). One can derive the relation between 


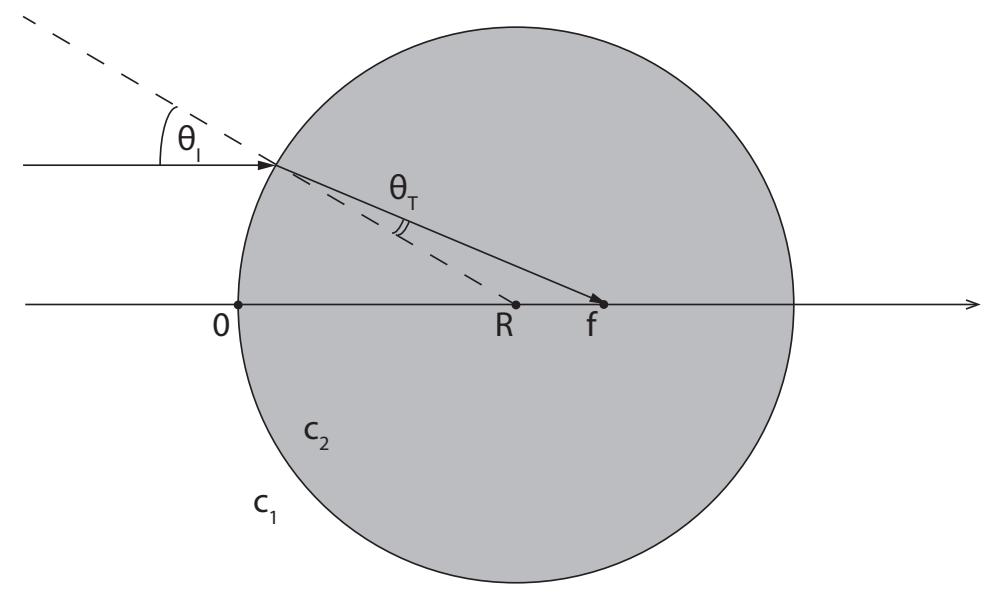

Figure 2.2: Schematics of the focusing of an acoustic wave on the droplet sphere when the acoustic wavelength is much larger than the droplet radius.

these angles by considering continuity of the normal displacement at the interface. This gives Snell's reflection law $\sin \theta_{\mathrm{I}}=\sin \theta_{\mathrm{R}}$ and $c_{2} \sin \theta_{\mathrm{I}}=\mathrm{c}_{1} \sin \theta_{\mathrm{T}}$, where $c_{1}$ and $c_{2}$ are the speed of sound of the first and the second medium respectively [26].

\subsubsection{Focusing inside a spherical droplet}

When an interface between two acoustic media has a finite curvature $R$, as in the case of a spherical droplet, acoustic focusing is observed. This is a similar effect as the focusing of light by an optical lens. First, the case of large droplets is considered, i.e. when the acoustic wavelength $\lambda$ is much smaller than the droplet radius $R$. Next, the case where $\lambda$ is of the order of $R$, or even larger, is considered.

\section{Case 1: droplets much larger in size than the wavelength}

When $\lambda \ll R$, the refraction formulas provided by the theory of geometrical scattering apply. When a parallel beam of light travels in a medium with refractive index $n_{1}$ and encounters a spherical interface between this medium and a second medium with refractive index $n_{2}$, either the transmitted or the 
reflected wave focuses in a point at a distance:

$$
f=R \frac{n_{2}}{n_{2}-n_{1}} .
$$

This distance is measured from the intersection point of the interface and the beam axis, which crosses the center of the curvature (Fig. 2.2). In analogy with the optical focus, an acoustic focus can be calculated for the case $\lambda \ll R$ by simply replacing $n_{1} / n_{2}$ with $c_{2} / c_{1}$ in the equation above. This gives:

$$
f=R \frac{c_{1}}{c_{1}-c_{2}} \text {. }
$$

For instance, when a large spherical perfluoropentane droplet with $c_{2}=406 \mathrm{~m} / \mathrm{s}$ is immersed in water with $\mathrm{c}_{1}=1522 \mathrm{~m} / \mathrm{s}$ (at $37^{\circ} \mathrm{C}$ ), the acoustic focus is at $f=1.36 R$. This means that the acoustic wave focuses on a distal side, $0.36 R$ away from the geometrical droplet center.

\section{Case 2: droplets similar or smaller in size than the wavelength}

The situation complicates when the radius of the droplet is of the same order of magnitude as the wavelength, or even smaller, i.e. when $\lambda \sim R$ or $\lambda \gg$ R. Experimental data obtained with small droplets $[4,36]$ shows that the ultrasound beam focuses on the proximal side of the droplet, which is not in agreement with the prediction above. Obviously, in this case geometrical considerations can no longer be applied, and a full wave theory must be applied. Figure 2.3 shows the configuration of the acoustic diffraction problem that will be solved here. Throughout the derivations, the parameters of the surrounding medium are labeled with a subscript 1 , and the parameters of the medium inside the droplet are labeled with a subscript 2.

At the location of the droplet, the incident ultrasound wave is considered to be planar. In view of Eq. 2.9, it is written as:

$$
p_{i}(x, y, z, t)=\sum_{n=0}^{\infty} a_{n} e^{i\left(n \omega t-n k_{1} z+\phi_{n}\right)},
$$

where $k_{1}=\omega / c_{1}$ is the wave number outside the droplet. To keep the derivations simple, the diffraction problem will first be solved for one spectral component:

$$
p_{i}(x, y, z, t)=a e^{i\left(\omega t-k_{1} z+\phi\right)} .
$$




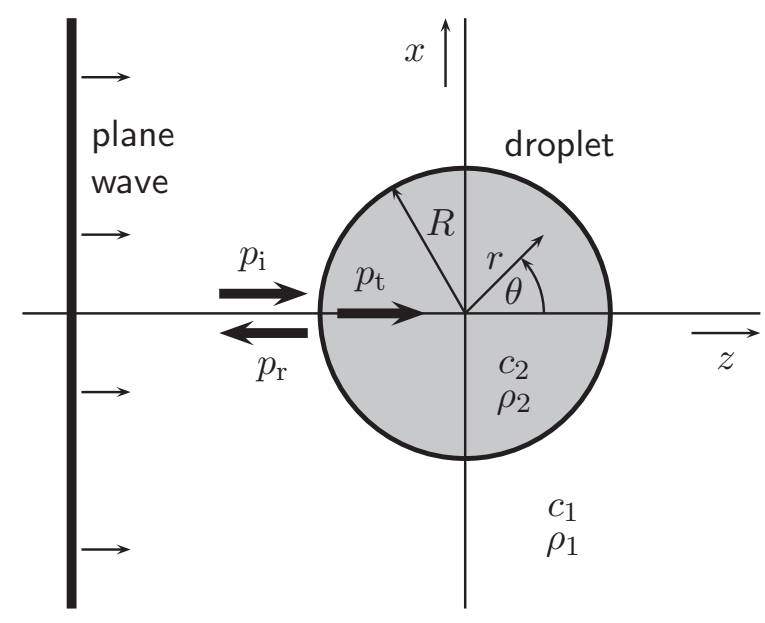

Figure 2.3: Configuration of the droplet and the incident, transmitted, and reflected ultrasound waves.

In view of the spherical symmetry of the configuration, it is convenient to apply a coordinate transformation from cartesian coordinates $(x, y, z)$ to spherical coordinates $(r, \theta, \varphi)$, where $\theta$ is the azimuthal angle measured with respect to the positive $z$-axis, and $\varphi$ is the elevation angle in the $x y$-plane. Because the waves will have rotational symmetry with respect to the $z$-axis, there will be no dependence on $\varphi$ and this coordinate will be omitted. In spherical coordinates, the incident pressure wave can be written as a summation of spherical harmonics:

$$
p_{i}(r, \theta, t)=a e^{i(\omega t+\phi)} \sum_{m=0}^{\infty} \gamma_{m} j_{m}\left(k_{1} r\right) P_{m}(\cos \theta),
$$

Here, $\gamma_{m}=(2 m+1)(-i)^{m}, j_{m}$ is the spherical Bessel function of the first kind and order $m$, and $P_{m}$ is the $m$-th order Legendre polynomial. The spherical Bessel function $j_{m}$ is related to the ordinary Bessel function $J_{m}$ according to $j_{\mathfrak{m}}(x)=\sqrt{(\pi / 2 x)} J_{\mathfrak{m}+1 / 2}(x)$.

When the incident wave encounters the droplet, it gives rise to a transmitted wave inside to droplet:

$$
p_{t}(r, \theta, t)=a e^{i(\omega t+\phi)} \alpha_{m} j_{m}\left(k_{2} r\right) P_{m}(\cos \theta),
$$

and a reflected wave outside the droplet:

$$
p_{r}(r, \theta, t)=a e^{i(\omega t+\phi)} \beta_{m} h_{m}^{(2)}\left(k_{1} r\right) P_{m}(\cos \theta) .
$$


In these equations, $k_{2}=\omega / c_{2}$ is the wave number inside the droplet, and $h_{m}^{(2)}$ is the spherical Hankel function of the second kind and order $m$. The spherical Hankel function $h_{m}^{(2)}$ is related to the ordinary Hankel function $\mathrm{H}_{\mathrm{m}}^{(2)}$ following $h_{m}^{(2)}(x)=\sqrt{(\pi / 2 x)} H_{m+1 / 2}^{(2)}(x)$. At the spherical interface between the outside and the inside of the droplet, the pressure and the radial particle velocity should be continuous. The latter requirement can be translated into a condition on the radial derivative of the pressure. In mathematical form, the boundary conditions at the interface are:

$$
\begin{aligned}
\lim _{r \downarrow R}\left[p_{i}(r, \theta, t)+p_{r}(r, \theta, t)\right] & =\lim _{r \uparrow R} p_{t}(r, \theta, t), \\
\lim _{r \downarrow R} \frac{1}{\rho_{1}} \frac{\partial}{\partial r}\left[p_{i}(r, \theta, t)+p_{r}(r, \theta, t)\right] & =\lim _{r \uparrow R} \frac{1}{\rho_{2}} \frac{\partial}{\partial r} p_{t}(r, \theta, t),
\end{aligned}
$$

which should hold for all $\theta$ and t. Substitution of Eqs. 2.42-2.44 into these boundary conditions results in a system of two equations for $\alpha_{m}$ and $\beta_{m}$. Solution of this system yields:

$$
\begin{aligned}
& \alpha_{m}=\gamma_{m} \frac{Z_{2} j_{m}\left(k_{1} R\right) h_{m}^{(2) \prime}\left(k_{1} R\right)-Z_{2} h_{m}^{(2)}\left(k_{1} R\right) j_{m}^{\prime}\left(k_{1} R\right)}{Z_{2} j_{m}\left(k_{2} R\right) h_{m}^{(2) \prime}\left(k_{1} R\right)-Z_{1} h_{m}^{(2)}\left(k_{1} R\right) j_{m}^{\prime}\left(k_{2} R\right)}, \\
& \beta_{m}=\gamma_{m} \frac{Z_{1} j_{m}\left(k_{1} R\right) j_{m}^{\prime}\left(k_{2} R\right)-Z_{2} j_{m}\left(k_{2} R\right) j_{m}^{\prime}\left(k_{1} R\right)}{Z_{2} j_{m}\left(k_{2} R\right) h_{m}^{(2) \prime}\left(k_{1} R\right)-Z_{1} h_{m}^{(2)}\left(k_{1} R\right) j_{m}^{\prime}\left(k_{2} R\right)},
\end{aligned}
$$

where $Z_{1}=\rho_{1} c_{1}$ and $Z_{2}=\rho_{2} c_{2}$ are the acoustic impedances of the media outside and inside the droplet, respectively. The prime indicates the derivative of a function. The constant $\alpha_{m}$ can be considered as the transmission coefficient of the droplet interface for spherical harmonics of order $m$, and the constant $\beta_{\mathrm{m}}$ can be considered as the corresponding reflection coefficient. At this stage, the problem of finding the wave inside the droplet due to a single sinusoidal component of the incident wave, is solved.

To find the wave that is formed inside the droplet by the nonlinear incident wave, all the transmitted waves caused by the individual components of the incident wave must be added. The result is:

$p_{\text {inside }}(r, \theta, t)=p_{t}(r, \theta, t)=\sum_{n=0}^{\infty} \sum_{m=0}^{\infty} a_{n} e^{i\left(n \omega t+\phi_{n}\right)} \alpha_{n, m} j_{m}\left(n k_{1} r\right) P_{m}(\cos \theta)$,

where $\alpha_{n, m}$ follows from Eq. 2.47 by replacing $k_{1}$ by $n k_{1}$ and $k_{2}$ by $n k_{2}$. This equation can be used to calculate the pressure in any position $(r, \theta, \phi)$ 
at any time $t$. Numerical implementation requires that both summations involve a finite number of terms. This forms no significant limitation, because in practice only a limited number of $\mathrm{N}$ harmonics will give a significant contribution to the ultrasound field inside the droplet, and only a limited number of $\mathrm{M}$ spherical harmonics is required to accurately represent this field. However, another numerical issue arises when the radius of the droplet is much smaller than the wavelength. In this case the numerical results for the spherical Bessel and Hankel functions may contain large errors. This problem may be eliminated by first approximating these functions by their series expansion around zero. With the full pressure field determined both in space and time we can now find the local maximum of pressure - the focus.

The pressure amplification factor in the focusing spot as well as its location depend on the input parameter values, i.e. the pressure amplitude, the frequency, and the transducer geometry and size, which prescribe the focusing strength and the propagation distance to the focal point. For instance, in case of a $R=10 \mu \mathrm{m}$ perfluoropentane droplet immersed in water and insonified with an incoming ultrasound wave with a peak negative pressure $P_{i}^{-}=-4.5 \mathrm{MPa}$ and frequency $\mathrm{f}=3.5 \mathrm{MHz}\left(\lambda=430 \mu \mathrm{m}\right.$ in water at $\left.37^{\circ} \mathrm{C}\right)$ coming from a transducer with $3.81 \mathrm{~cm}$ focal distance, a focused peak negative pressure of $\mathrm{P}_{\text {inside }}^{-}=-26 \mathrm{MPa}$ is achieved within the droplet. Thus, a near six-fold increase in the peak negative pressure amplitude is observed in a concentrated region on the proximal side around $z=-0.4 \mathrm{R}$.

From Eq. 2.47 it follows that the pressure inside the droplet, due to a single incident wave component, depends on the dimensionless product $\omega R$. When for two droplets with different radii $R_{1}$ and $R_{2}$ the relation $\omega_{1} R_{1}=\omega_{2} R_{2}$ holds, an incident wave with frequency $f_{1}$ encountering a droplet with radius $R_{1}$ is focused at the same relative position within the droplet as an incident wave with frequency $f_{2}$ that hits a droplet with radius $R_{2}$. This implies that when larger droplets turn out to vaporize more easily than smaller droplets at the same frequency, it also follows that for the same radius droplets are easier to evaporate at high frequencies than at low frequencies, and vise versa.

However, nonlinear propagation makes this picture more complex. First, the higher the acoustic pressure amplitude, the more nonlinear the wave becomes, as the amplitudes of the higher harmonics build up roughly as $\left(\mathrm{P}_{\text {surface }}\right)^{\mathrm{n}}$, where $P_{\text {surface }}$ is the pressure amplitude at the transducer surface and $n$ is the number of the particular harmonic. Second, the nonlinear propagation depends on the frequency. And, of course, the nonlinear beam is focused differently from the linear one, with different pressure amplification factors and 
focusing positions for each harmonic. Finally, the shape of the nonlinearly distorted wave is strongly dependent on the parameters of the propagating media. For human tissue the Goldberg ratio is lower than for water [37]. This indicates that nonlinear distortion is easier to achieve in water, compared to tissue. Therefore the experiments performed in vivo are expected to have different nucleation patterns, with a higher nucleation threshold compared to the in vitro experiments.

Knowledge of the physics of acoustic focusing in small droplets is important for the optimization of acoustic droplet vaporization for therapeutic applications. This is particularly the case for attaining activation at low acoustic pressures, thereby minimizing the negative bio-effects associated with the use of high-intensity ultrasound. Moreover, it helps in the design of droplets: by mixing liquids with different physical properties, the acoustic impedance may be tuned trough a change of the density of mass and/or the speed of sound. Using dedicated waveforms, the amplitudes and phases of the nonlinear wave at the focus of the beam can be optimized to obtain maximal constructive interference within the droplets and obtain maximal focusing strength at any particular acoustic input pressure. Moreover the knowledge of the consecutive droplet vaporization dynamics is important, because it affects the surrounding tissue and may cause damage.

Not only the acoustic impedance mismatch between the droplet and the surrounding media determines the interior pressure, but also the exterior of the droplet. Here we have only considered single droplets. But clouds of droplets may cause complicated pressure scattering patterns and may lead to different focusing spots as compared to the single droplet case. One can also think of periodic arrangements of monodisperse droplets, to observe similar diffraction relations as we have in light passing through crystals (see Chapter 8).

\subsubsection{Radial vapor bubble expansion}

There are three main physical mechanisms that govern the vapor bubble growth process: phase-change, heat transfer and inertia. And there are two phenomena which can limit the vapor bubble growth. First, the vapor bubble pushes the surrounding liquid as it grows. The force by which is pushed out the liquid apart is determined by the pressure which acts on the bubble wall. The surrounding liquid has inertia, and the vapor bubble growth rate will be limited by this inertia. Second, the phase-change from liquid to vapor is an en- 
dothermic process which requires heat to be absorbed. The required heat for vaporization is transfered from the liquid around the bubble by cooling the surrounding. The rate of this process is limited by the heat transfer.

Let us now first have a closer look on the inertial growth limitation. Here we assume that the heat transfer is high enough to supply the required energy for the endothermic phase-transition. In this case the Rayleigh-Plesset equation can be written as:

$$
\ddot{R} R+\frac{3}{2} \dot{R}^{2}=\frac{P_{V}-P_{\infty}}{\rho},
$$

where $P_{V}$ is the vapor pressure and $P_{\infty}$ is the pressure far away from the bubble wall. We disregard the surface tension, the sound reradiation and the viscosity. The boiling temperature of the liquid is $T_{b}$ and the ambient temperature is $T_{\infty}$. The liquid is superheated $\left(T_{\infty}>T_{b}\right)$ so that $P_{V}>P_{\infty}$. The vapor pressure $P_{V}$ is a function of the temperature and assumed to be constant during the vapor bubble growth. Initially the velocity of the bubble wall $\dot{R}$ is small, and the first term on the left hand side of Eq. 2.50 is dominant. After approximately a few nanoseconds at $\mathrm{P}_{V}=1.4 \mathrm{P}_{\infty}$ the bubble wall velocity reaches its terminal value and the second term on the right hand side of Eq. 2.50 becomes dominant. Terminal velocity is reached at the condition $\ddot{R} \rightarrow 0$. Substituting this into Eq. 2.50 and integrating with the initial condition $R(t=0)=0$ gives the radius-time dependency of the inertially limited vapor bubble growth:

$$
R(t)=\left(\frac{2}{3} \frac{\left(P_{V}-P_{\infty}\right)}{\rho}\right)^{1 / 2} t
$$

Eq. 2.51 is linear with time and is faster for higher vapor pressures $\mathrm{P}_{\mathrm{V}}$, thus at higher ambient temperatures $T_{\infty}$.

Let us now have a look at the second case, where we focus on the heat transfer and the inertial limitations are neglected. Contrary to the solution of the inertial problem, the heat transfer is complicated by the temperature distribution outside the vapor bubble (Fig. 2.4). The temperature distribution changes with time due to thermal diffusion. In addition, it is also affected by the expansion of the bubble, described by the continuity equation. The effective thermal boundary layer around the vapor bubble is determined by [33]:

$$
\delta_{\mathrm{eff}}=\sqrt{\mathrm{Dt}}
$$




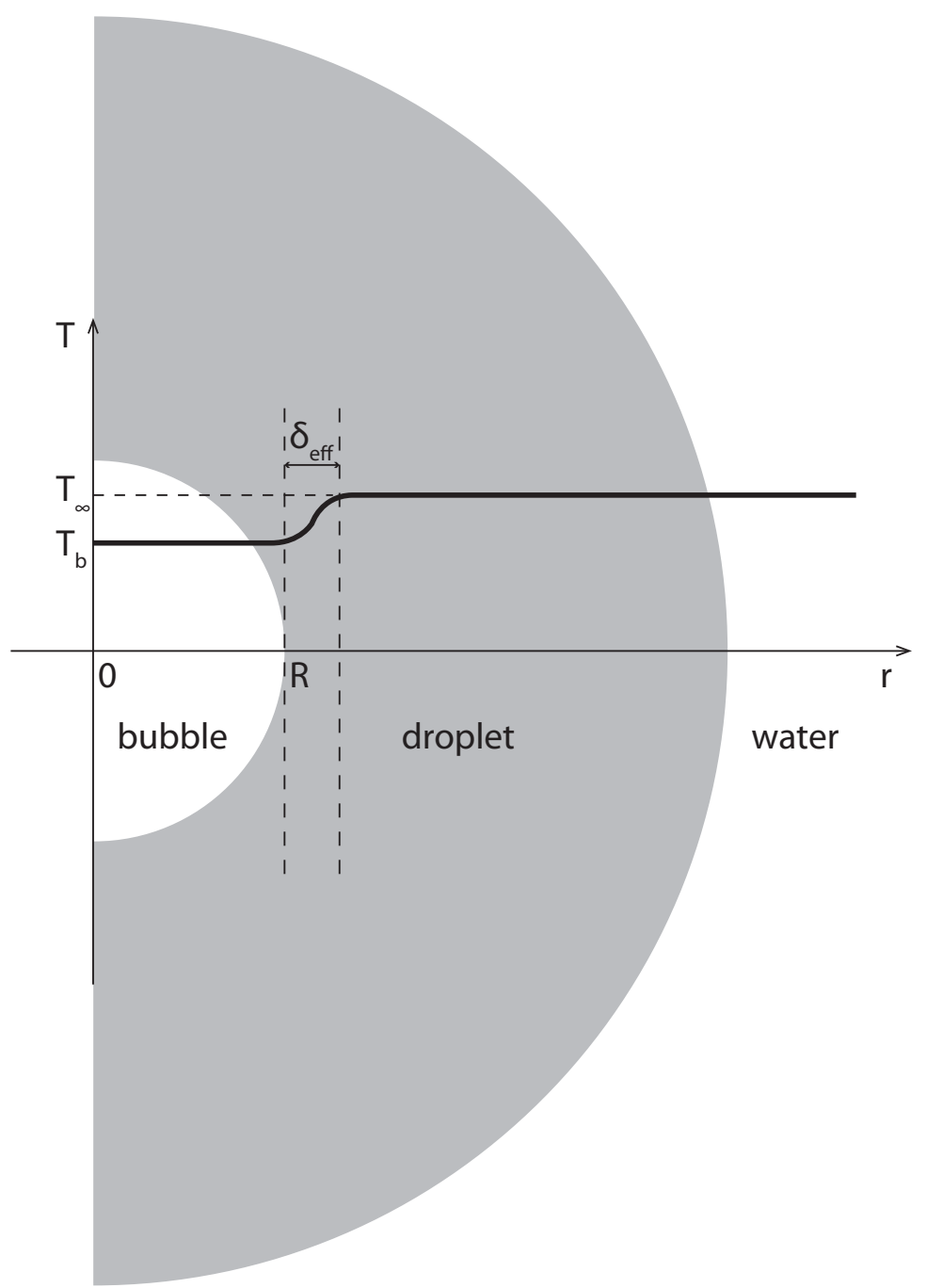

Figure 2.4: Schematics of the temperature distribution during the vaporization of superheated perflourocarbon droplet immersed in water. $\mathrm{T}_{\mathrm{b}}$ is boiling temperature of perfluorocarbon, $\mathrm{T}_{\infty}$ is ambient temperature, and $\delta_{\mathrm{eff}}$ is effective thermal boundary layer around the vapor bubble of the radius $\mathrm{R}$. 
where $\mathrm{D}$ is the thermal diffusivity of the liquid. This estimation follows from the thermal diffusion equation and shows that the thermal boundary layer diffuses with time as $\sqrt{t}$. On the vapor side of the thermal boundary layer the temperature is $T_{b}$, and on the liquid side of the thermal boundary layer the temperature is $T_{\infty}$. The effective temperature gradient over the thermal boundary layer is $\Delta \mathrm{T} / \delta_{\text {eff }}$, where $\Delta \mathrm{T}=\mathrm{T}_{\infty}-\mathrm{T}_{\mathrm{b}}$ is the temperature difference. The heat flow $W_{1}$ inside the vapor bubble from the surrounding liquid caused by the temperature mismatch can be estimated as follows:

$$
W_{1}=4 \pi R^{2} k \frac{\Delta T}{\sqrt{D t}},
$$

where $k$ is the heat transfer coefficient and $4 \pi R^{2}$ is the interfacial area.

The latent heat energy per unit time $W_{2}$ required to supply the vapor bubble growth is:

$$
W_{2}=4 \pi R^{2} L \rho_{v} \frac{d R}{d t}
$$

with $L$ the latent heat, and $4 \pi R^{2} \rho_{v} d R / d t$ is the derivative of the mass with $\rho_{\mathrm{v}}$ the density of the vapor. Equalizing Eqs. 5.5 and 5.6 and integrating with the initial condition $R(t=0)=0$ gives the radial dynamics of the heat transfer limited vapor bubble growth:

$$
R(t)=2 \frac{k \Delta T}{L \rho_{v} \sqrt{D}} \sqrt{t},
$$

it is dependent on time $t$ following a square root behavior and eventually will become slower than the inertia limited vapor bubble growth expressed by the linear dependence by Eq. 2.51. Thus, initially the vapor bubble growth is limited by the inertia, then the vapor bubble growth becomes limited by the heat transfer. One can estimate the radius and the time when the transition of the two regimes occurs by calculating the intersection of the two curves expressed by the Eqs. 2.51 and 2.55. For the typical parameters of the acoustic perfluorocarbon droplet vaporization the vapor bubble growth is heat transfer limited for a typical timescale longer than 1 microsecond.

When the bubble growth is accompanied by bubble oscillations due to the ultrasound forcing, one can observe a phenomenon called rectified heat transfer. Rectified heat transfer is the net effect of the decrease of the heat transfer during the ultrasound half cycle when the vapor bubble surface contracts, and which is lower than the increase of the heat transfer during the 
second half cycle when the surface expands. Here two effects come into play. First, the increment of the bubble wall area during the expansion cycle, and second, the increment of the temperature gradient. The increment of the temperature gradient can be understood in the following way. Let us consider that the radius of the vapor bubble changes from $R_{0}$ to $R$. The change of the thin thermal boundary layer from $\delta_{0}$ to $\delta$ is then calculated from continuity: $4 \pi R^{2} \delta=4 \pi R_{0}^{2} \delta_{0}$. This gives [33]:

$$
\delta=\delta_{0} \frac{R_{0}^{2}}{R^{2}}
$$

And from the reciprocal relation for the temperature gradient:

$$
\frac{\Delta \mathrm{T}}{\delta}=\frac{\Delta \mathrm{T}}{\delta_{0}} \frac{\mathrm{R}^{2}}{\mathrm{R}_{0}^{2}},
$$

it can be seen that the temperature gradient increases with the radius squared $R^{2}$, i.e. with the bubble wall area $4 \pi R^{2}$. Thus, the bubble wall area and the temperature gradient will both decrease with $R^{2}$ when the radius decreases. However the net effect is typically positive, meaning that bubble wall oscillations due to the interaction with ultrasound will pump additional heat into the bubble, thereby promoting the phase-conversion process. The larger the bubble oscillation amplitude, the stronger the pumping of additional heat.

\subsubsection{Activation below boiling point}

After the initiation of droplet vaporization by the focused ultrasound pulse, gas diffuses into the nucleus/vapor bubble during the vapor bubble growth, as perfluorocarbon droplets dissolve air by an order of magnitude more than water. As was shown before, vapor bubble growth strongly depends on the temperature. From both Eq. 2.51 and Eq. 2.55 it follows that the vapor bubble growth is slower when the ambient temperature is lower, whereas the dependence of air diffusion on the temperature is much less pronounced. This means that at low ambient temperatures $\left(T_{\infty} \leqslant T_{b}\right)$ the air diffusion dynamics becomes comparable to the evaporation processes.

Here, for simplicity, we only show the bubble growth dynamics due to gas diffusion, disregarding the evaporation processes and oscillations of the bubble due to ultrasound forcing. The partial pressure of gas $P_{g}$ which is in equilibrium with the saturated gas concentration $c_{s}$ dissolved in the liquid is given by Henry's law: 


$$
\mathrm{P}_{\mathrm{g}}=\mathrm{Hc}_{\mathrm{s}}
$$

We assume that the liquid is at a uniform supersaturated concentration $c_{i}$. The mass flow of gas into the bubble per unit time is:

$$
\frac{\mathrm{dm}}{\mathrm{dt}}=\left.4 \pi \mathrm{R}^{2} \mathrm{~K} \frac{\partial \mathrm{c}}{\partial \mathrm{r}}\right|_{\mathrm{r}=\mathrm{R}},
$$

where $\kappa$ is the coefficient of diffusivity of the gas in the liquid. If $\rho_{g}$ is the density of the gas in the bubble, the mass flow can be written as follows:

$$
\frac{d m}{d t}=4 \pi R^{2} \rho \frac{d R}{d t}
$$

One can use the reasonable physical approximation to calculate the gradient of the concentration for a bubble interface which changes in time by diffusion [38]:

$$
\left.\frac{\partial c}{\partial r}\right|_{r=R}=\left(c_{i}-c_{s}\right)\left(\frac{1}{R}+\frac{1}{\sqrt{\pi k t}}\right)
$$

Substitution of Eq. 2.60 and Eq. 2.62 into Eq. 2.59 gives the radial time dynamics equation for the gas diffusion:

$$
\frac{d R}{d t}=\frac{\kappa\left(c_{i}-c_{s}\right)}{\rho}\left(\frac{1}{R}+\frac{1}{\sqrt{\pi \kappa t}}\right) .
$$

The gas bubble shrinks when $c_{i}<c_{s}$ and grows when $c_{i}>c_{s}$. Similar to the rectified heat transfer problem, gas diffusion into the bubble can be promoted due to interaction with ultrasound. This phenomenon is called rectified diffusion and similar relations can be derived as was shown in the previous subsection.

\section{References}

[1] O. D. Kripfgans, J. B. Fowlkes, D. L. Miller, O. P. Eldevik, and P. L. Carson, "Acoustic droplet vaporization for therapeutic and diagnostic applications", Ultrasound Med. Biol. 26, 1177-1189 (2000). 
[2] N. Reznik, O. Shpak, E. C. Gelderblom, R. Williams, N. de Jong, M. Versluis, and P. N. Burns, "The efficiency and stability of bubble formation by acoustic vaporization of submicron perfluorocarbon droplets", Ultrasonics 53, 1368-1376 (2013).

[3] O. Shpak, L. Stricker, M. Versluis, and D. Lohse, "The role of gas in ultrasonically driven vapor bubble growth", Phys. Med. Biol. 58, 25232535 (2013).

[4] O. Shpak, T. Kokhuis, Y. Luan, D. Lohse, N. de Jong, B. Fowlkes, M. Fabiilli, and M. Versluis, "Ultrafast dynamics of the acoustic vaporization of phase-change microdroplets", J. Acoust. Soc. Am. 134, 1610-1621 (2013).

[5] T. Giesecke and K. Hynynen, "Ultrasound-mediated cavitation thresholds of liquid perfluorocarbon droplets in vitro", Ultrasound Med. Biol. 29, 1359-1365 (2003).

[6] K. C. Schad and K. Hynynen, "In vitro characterization of perfluorocarbon droplets for focused ultrasound therapy", Phys. Med. Biol. 55, 4933-4947 (2010).

[7] R. Williams, C. Wright, E. Cherin, N. Reznik, M. Lee, I. Gorelikov, F. S. Foster, N. Matsuura, and P. N. Burns, "Characterization of submicron phase-change perfluorocarbon droplets for extravascular ultrasound imaging of cancer", Phys. Med. Biol. 39, 475-489 (2013).

[8] D. T. Blackstock, "On plane, spherical and cylindrical sound waves of finite amplitude in lossless fluids", J. Acoust. Soc. Am. 36, 217-219 (1964).

[9] D. R. Bacon, "Finite amplitude distortion of the pulsed fields used in diagnostic ultrasound", Ultrasound Med. Biol. 10, 189-195 (1984).

[10] P. Westervelt, "Parametric acoustic array", J. Acoust. Soc. Am. 52, 535537 (1963).

[11] M. Hamilton and C. Morfey, Model equations. In: Hamilton, M.F. and Blackstock, D.T. (eds.) Nonlinear acoustics. pp 41-63 (Melville, NY: Acoustical Society of America) (2008). 
[12] D. Lee and A. Pierce, "Parabolic equation development in recent decade", J. Comput. Acoust. 3, 95-173 (1995).

[13] E. A. Zabolotskaya and R. V. Khokhlov, "Quasi-plane waves in the nonlinear acoustics of confined beams", Sov. Phys. Acoust. 15, 35-40 (1969).

[14] V. P. Kuznetsov, "Equation of nonlinear acoustics", Sov. Phys. Acoust. 16, 467-470 (1971).

[15] M. Hamilton, J. N. Tjotta, and S. Tjotta, "Nonlinear effects in the farfield of a directive sound source", J. Acoust. Soc. Am. 78, 202-216 (1985).

[16] T. S. Hart and M. F. Hamilton, "Nonlinear effects in focused sound beams", J. Acoust. Soc. Am. 84, 1488-1496 (1988).

[17] T. Kamakura, T. Ishiwata, and K. Matsuda, "Model equation for strongly focused finite-amplitude sound beams", J. Acoust. Soc. Am. 107, 30353046 (2000).

[18] Y.-S. Lee and M. F. Hamilton, "Time-domain modeling of pulsed finiteamplitude sound beams", J. Acoust. Soc. Am. 97, 906-917 (1995).

[19] R. Cleveland, M. Hamilton, and D. T. Blackstock, "Time-domain modeling of finite-amplitude sound in relaxing fluids", J. Acoust. Soc. Am. 99, 3312-3318 (1996).

[20] T. Varslot and G. Taraldsen, "Computer simulation of forward wave propagation in soft tissue", IEEE Trans. Ultrason. Ferroelec. Freq. Contr. 52, 1473-1482 (2005).

[21] L. Rayleigh, "On the pressure development in a liquid during the collapse of a spherical cavity", Philos. Mag. 32 (S8), 94 - 98 (1917).

[22] M. S. Plesset, "The dynamics of cavitation bubbles", J. Appl. Phys. 16, 277282 (1949).

[23] B. E. Noltingk and E. A. Neppiras, "Cavitation produced by ultrasonics”, Proceedings of the Physical Society. Section B 63, 674-685 (1950).

[24] E. A. Neppiras and B. E. Noltingk, "Cavitation produced by ultrasonics: Theoretical conditions for the onset of cavitation", Proceedings of the Physical Society. Section B 64, 1032-1038 (1951). 
[25] H. Poritsky, "The collapse or growth of a spherical bubble or cavity in a viscous fluid", Proceedings of the first US National Congress on Applied Mechanics 813-821 (1952).

[26] T. G. Leighton, The acoustic bubble (Academic, London, 1994).

[27] P. Marmottant, S. M. van der Meer, M. Emmer, M. Versluis, N. de Jong, S. Hilgenfeldt, and D. Lohse, "A model for large amplitude oscillations of coated bubbles accounting for buckling and rupture", J. Acoust. Soc. Am. 118, 3499-3505 (2005).

[28] N. de Jong, M. Emmer, C. T. Chin, A. Bouakaz, F. Mastik, D. Lohse, and M. Versluis, "“Compression-Only” behavior of phospholipid-coated contrast bubbles", Ultrasound Med. Biol. 33 (2007).

[29] C. C. Church, "The effects of an elastic solid surface layer on the radial pulsations of gas bubbles", J. Acoust. Soc. Am. 97, 1510-1521 (1995).

[30] M. Overvelde, V. Garbin, J. Sijl, B. Dollet, N. de Jong, D. Lohse, and M. Versluis, "Nonlinear shell behavior of phospholipid-coated microbubbles", Ultrasound Med. Biol. 36, 2080-2092 (2010).

[31] M. Overvelde, "Ultrasound Contrast Agents: Dynamics of coated bubbles", Ph.D. thesis (2010).

[32] M. Minnaert, "On musical air-bubbles and sounds of running water", Philosophical Magazine 16, 235-248 (1933).

[33] A. Prosperetti, Advanced Mathematics for Applications (Cambridge University Press) (2011).

[34] V. F. K. Bjerknes, Fields of Force (Columbia University Press) (1906).

[35] T. G. Leighton, The Acoustic Bubble (Academic Press) (1994).

[36] O. Shpak, M. Verweij, R. Vos, N. de Jong, D. Lohse, and M. Versluis, "Acoustic droplet vaporization is initiated by superharmonic focusing", Proc. Natl. Acad. Sci. 111, 1697-1702 (2014).

[37] T. L. Szabo, F. Clougherty, and C. Grossman, "Effects on nonlinearity on the estimation of in situ values of acoustic output parameters", Ultrasound Med. Biol. 18, 33-42 (1999). 
[38] P. S. Epstein and M. S. Plesset, "On the stability of gas bubbles in liquidgas solutions", The Journal of Chem. Phys. 18, 1505-1509 (1950). 


\section{3 \\ Nanodroplets $\ddagger^{\ddagger}$}

Submicron droplets of liquid perfluorocarbon converted into microbubbles with applied ultrasound have been studied, for a number of years, as potential next generation extravascular ultrasound contrast agents. In this work, we conduct an initial ultra-high-speed optical imaging study to examine the vaporization of submicron droplets and observe the newly created microbubbles in the first microseconds after vaporization. It was estimated that single pulses of ultrasound at $10 \mathrm{MHz}$ with pressures within the diagnostic range are able to vaporize on the order of at least $10 \%$ of the exposed droplets. However, only part of the newly created microbubbles survives immediately following vaporization the bubbles may recondense back into the liquid droplet state within microseconds of nucleation. The probability of bubble survival within the first microseconds of vaporization was shown to depend on ultrasound excitation pressure as well as on bubble coalescence during vaporization, a behavior influenced by the presence of coating material on the newly created bubbles. The results of this study show for the first time that although initial

${ }^{\ddagger}$ Published as: Nikita Reznika, Oleksandr Shpak, Erik C. Gelderblom, Ross Williams, Nico de Jong, Michel Versluis, and Peter N. Burns, "The efficiency and stability of bubble formation by acoustic vaporization of submicron perfluorocarbon droplets", Ultrasonics $\mathbf{5 3}$ (7). 1368-1376 (2013).

*O. Shpak contributed to the analysis, experiments and discussions. 
vaporization of droplets is necessary to create echogenic bubbles, additional factors, such as coalescence and bubble shell properties, are important and should be carefully considered for the production of microbubbles for use in medical imaging.

\subsection{Introduction}

Liquid perfluorocarbon (PFC) droplets have been investigated for a number of years as a new generation of ultrasound (US) contrast agents [1]. Droplets filled with a relatively low-boiling point perfluorocarbon, such as perfluoropentane (PFP), stay in their liquid form at $37^{\circ} \mathrm{C}$ until exposed to ultrasound at sufficiently high rarefactional pressures [2], at which point the droplets vaporize into bubbles of gas. This unique property of selective acoustic activation makes the droplets a potentially useful tool for both diagnostic imaging and therapy [3]. Therapeutically, droplets have been studied as means for potentiating embolotherapy [2-4], aberration correction [5], HIFU sensitization [6-9] and drug delivery [10-13]. For diagnostic purposes, submicron perfluorocarbon droplets can be vaporized to produce micron-sized gas bubbles $[14,15]$. The bubbles, imaged within milliseconds of vaporization, were shown to be stable at the least on single-seconds timescales and to respond non-linearly to ultrasound, rendering them suitable for use as ultrasound contrast agents for both B-mode and contrast-specific imaging techniques such as pulse-inversion [14].

Droplets of diameters within the range of hundreds of nanometers have the ability to extravasate in regions of tumour growth, while staying intravascular in healthy tissues [12], due to the so-called enhanced permeability and retention effect [16]. This ability of the PFC droplets to passively target cancers may be used in order to selectively increase tumour contrast on an ultrasound image. Following this approach, direct detection of tumours with contrast enhanced US may be possible, unlike the current microbubble-based approaches used in clinical practice, which rely on irregular blood perfusion patterns of the hypervascularized tumours [17, 18].

Although recent studies have shown the potential for vaporized submicron PFC droplets as a contrast agent for diagnostic imaging [12, 14, 15], most work on nanodroplets has focused on the examination of the final bubble product at timescales ranging from milliseconds to seconds and minutes following ultrasound excitation. A number of high speed imaging studies [19- 
21] have been performed to examine the vaporization of micron sized droplets on the microsecond timescales. However, observations of vaporization and subsequent behavior of submicron (rather than the bigger micron-sized) PFC droplets on the microsecond and nanosecond timescales have not been performed the size dynamics, as well as presence of bubble fragmentation and coalescence have not been examined in the submicron droplets case.

The efficiency of conversion and stability of vaporized droplets is of utmost importance for successful application of the proposed technique for ultrasound imaging in a clinical setting the amount of droplets necessary to produce sufficient amount of detectable bubbles should be minimized. The efficiency of such conversion is dependent on the initial droplet nucleation rate as well as the stability of the newly created bubbles against recondensation back into the liquid form following vaporization. While it was shown the bubbles are stable on the single milliseconds to single seconds timescale [14, 15], little is known about their initial nucleation rate and their stability against recondensation, in the first few microseconds after phase conversion. Knowledge of bubble behavior in the first microseconds after vaporization is necessary to optimize bubble production techniques for their successful application on diagnostic imaging timescales (milliseconds to seconds).

In this work, we conduct an initial ultra-high speed imaging study of submicron droplet vaporization, to examine the newly created microbubbles in the first $10 \mu \mathrm{s}$ after vaporization. We consider droplets of liquid PFP of a few-hundred nanometers in diameter, stabilized by a charged fluorosurfactant and vaporized with single US pulses at pressures within the diagnostic range. We calculate a lower limit order of magnitude estimate of the ratio of droplets that undergo vaporization and examine the initial vaporization-induced size change of the bubbles as well as bubble coalescence, fragmentation and the recondensation of the bubbles back into the liquid state. We measure the dependence of probability of bubble recondensation on ultrasound excitation intensity and bubble coalescence. Finally, we discuss the possible mechanisms that influence bubble stability and bubble recondensation in the first microseconds following vaporization. 


\subsection{Theory}

\subsubsection{Initial droplet nucleation and PFC vapor pressure}

There are a number of general conditions that need to be satisfied in order for droplet vaporization to be energetically favourable. The process of bubble formation from homogeneous nucleation in an acoustic field is described by Church [22]. A nucleation bubble seed above a specific critical radius for vaporization, $r_{c v}$ is required for droplet vaporization. The value of $r_{c v}$ is given by:

$$
r_{c v}=-\frac{2 \sigma_{D}}{p_{h}-p_{v}+p_{a}}
$$

where $\sigma_{D}=9.5 \mathrm{mN} / \mathrm{m}$ is the surface tension of PFP (as provided by Fluoromed, Round Rock, TX, USA), ph is the hydrostatic pressure within the droplet, $p_{v}$ is the vapor pressure of the superheated PFC and $p_{a}$ is the instantaneous applied acoustic pressure. A seed of size above $r_{c v}$ may be incepted due to internal energy fluctuations within the liquid, in a stochastic manner. From the equation, it is apparent that vaporization is possible only when $p_{a}<p_{v}-p_{h}$.

$$
p_{v}=10^{A-B /(T+C)}
$$

where $\mathrm{T}$ is the temperature in Kelvin, and $\mathrm{A}, \mathrm{B}$ and $\mathrm{C}$ are model parameters. The appropriate parameters for PFP were obtained from the National Institute of Standards and Technology (USA), fitted from the data obtained by Barber and Cady [23]. The approximated $p_{v}$ of perfluoropentane for the temperature range of $25^{\circ} \mathrm{C}$ to $45^{\circ} \mathrm{C}$ is shown in Fig. 3.1. As expected, at its natural boiling temperature of $29^{\circ} \mathrm{C}, \mathrm{p}_{v}=100 \mathrm{kPa}$, while at $37^{\circ} \mathrm{C}, \mathrm{p}_{v}=135$ $\mathrm{kPa}$.

\subsubsection{Rate of bubble condensation}

Using the Homogeneous Nucleation Theory, it is also possible to estimate the probability of bubble condensation into the liquid droplet form following the initial vaporization. Similar to the process of vaporization, bubble condensation requires a nucleation seed of above a specific critical size for condensation rcc that will grow to convert the vapor bubble into a liquid droplet. The 


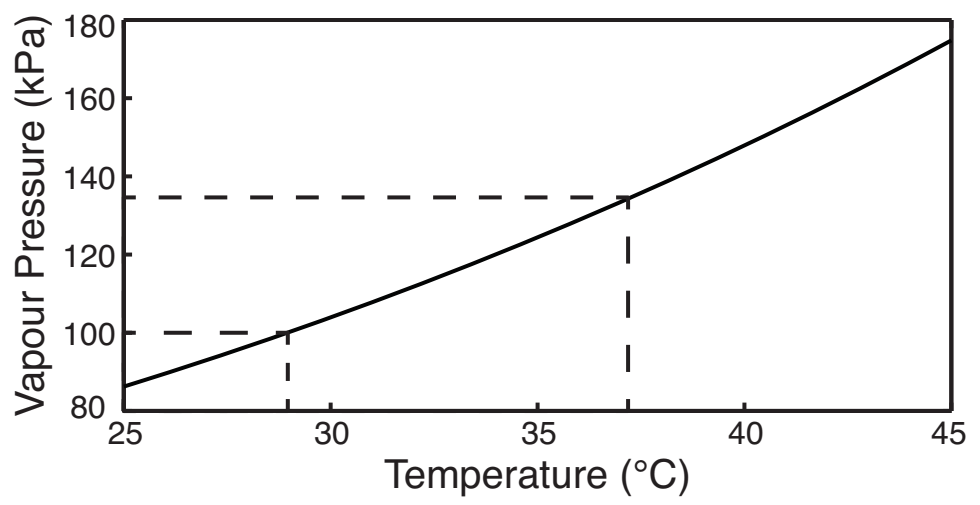

Figure 3.1: Vapor pressure in perfluoropentane as a function of temperature, as approximated by Antoine equation (Eq. 3.1). Highlighted are the vapor pressures at $29^{\circ} \mathrm{C}$, the natural boiling point of perfluoropentane, and $37^{\circ} \mathrm{C}$, the temperature at which the experiments were performed.

number of condensation events for a single bubble in a defined time period can be expressed as [24]:

$$
C=\frac{4 \pi p_{v} r_{c c}^{2}}{(2 \pi m k T)^{1 / 2}} \cdot \frac{P_{\text {bubble }}}{k T} \cdot \exp \left(\frac{16 \pi \sigma_{D}^{2} m^{2}}{k^{3} T^{3} \rho^{2} \ln ^{2}\left(P_{\text {bubble }} / p_{v}\right)}\right) \cdot V_{\text {bubble }} \cdot \Delta t
$$

where $C$ is the number of nucleation seeds above the critical cluster size $r_{c c}=\frac{2 \sigma_{D} m}{\rho k T h n\left(P_{b u b b l e} / p_{v}\right)}$ per observation, $P_{\text {bubble }}$ is the pressure inside the bubble, $k$ is the Boltzmann constant, $m$ and $\rho$ are the molecular mass and the density of PFC respectively, $V_{\text {bubble }}$ is the volume of the bubble, $\Delta t$ is the total time of the observation. By setting $C=1$ and solving numerically for $\mathrm{P}_{\text {bubble, }}$ it is possible to determine the condensation pressure threshold $P_{\text {cond. }}$ It should be noted that there is no significant difference in $P_{\text {cond }}$ for $C=1$ and for $C=10$. Fig. 3.2 shows the value of $P_{\text {cond }}$ for a PFP bubble at $37^{\circ} \mathrm{C}$ as a function of bubble radius $\mathrm{R}$ for an observation time period of $10 \mu \mathrm{s}$ and $1 \mathrm{~s}$. For comparison, $P_{\text {bubble }}$ of an uncoated bubble due to the atmospheric pressure and the Laplace pressure contributions is shown on the same graph. It is apparent that there is a threshold bubble radius $R_{\text {stable }}$ of approximately $800 \mathrm{~nm}$ above which the bubbles are stable against recondensation as $\mathrm{P}_{\text {bubble }}<\mathrm{P}_{\text {cond }}$. At the same time, uncoated bubbles below the size of $R_{\text {stable }}$ are prone to recondensation, as the internal pressure is sufficient to 


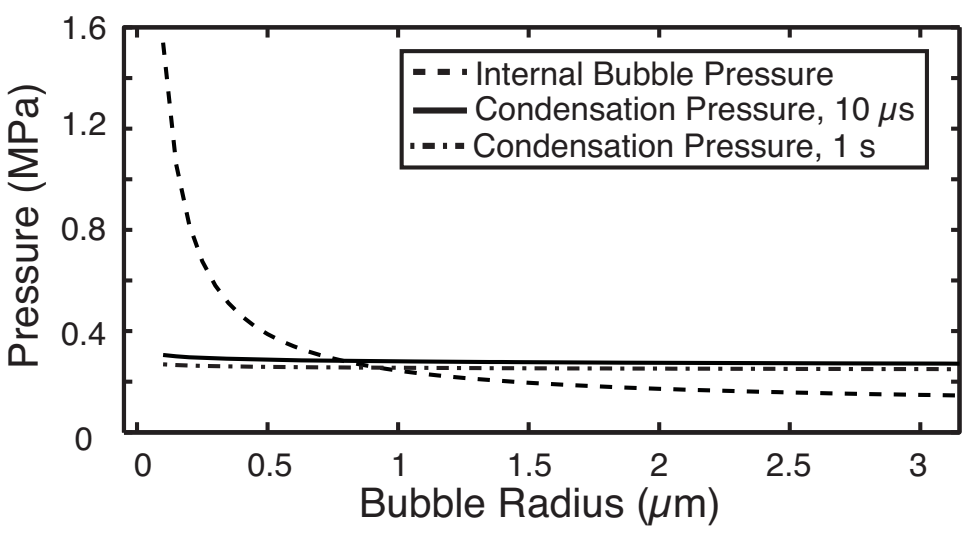

Figure 3.2: Necessary pressure to induce a condensation event $\mathrm{P}_{\mathrm{cond}}$ within a bubble within a time period of $10 \mu$ s (solid line) and $1 \mathrm{~s}$ (dashdotted line) as a function of bubble radius. Overlaid is the total internal pressure $P_{\text {bubble }}$ within an uncoated bubble surrounded by water due to atmospheric and Laplace pressure contributions (dashed line).

convert the bubble into the liquid droplet state. Increasing the waiting time does not significantly affect the threshold pressure for recondensation the change in $\mathrm{P}_{\text {cond }}$ for $10 \mu \mathrm{s}$ and $1 \mathrm{~s}$ time periods is only on the order of $50 \mathrm{kPa}$, which does not significantly affect the value of $R_{\text {stable }}$.

\subsubsection{Surface tension at the vaporized droplet interface}

Prior to vaporization, droplet emulsions are stabilized by a coating material, such as fluorosurfactant, as used in our study. It was previously shown qualitatively that, following vaporization and bubble formation, the newly created bubbles retain their initial coating material [20, 25]. In order to estimate the ability of this surfactant material to stabilize the bubble and prevent bubble recondensation back into the liquid form, we examine the coating material induced change of the effective surface tension in between the newly vaporized droplet and the surrounding liquid.

It was previously suggested [26] that for fluorinated surfactants the effective surface tension is dependent on the concentration of surfactant molecules on the bubble surface. For charged surfactants the surface tension may be expressed as follows [27]: 


$$
\sigma=\sigma_{0}-Q^{2} / 16 \pi^{2} \in \mathrm{R}^{3}
$$

where $\sigma_{0}=72 \mathrm{mN} / \mathrm{m}$ is the surface tension of water, $\mathrm{Q}$ is the total charge on the surface of the bubble, $\epsilon$ is the dielectric constant of the surrounding medium and $\mathrm{R}$ is the bubble radius. Each molecule of the charged surfactant carries a charge $\mathrm{q}$, and the total charge of the surfactant coating $\mathrm{Q}$ can be expressed as:

$$
\mathrm{Q}=\mathrm{qS}=\mathrm{q} \Gamma \cdot 4 \pi \mathrm{R}^{2}
$$

where $S$ is the total number of molecules of surfactant on the surface of the bubble, and $\Gamma$ is the concentration of surfactant molecules on the bubble surface as a function of the bubble radius R. From Eqs. (4) and (5) it follows, then, that:

$$
\sigma=\sigma_{0}-\frac{q^{2} \Gamma^{2} R}{\epsilon}=\sigma_{0}-\sigma_{s}(R)
$$

where $\sigma_{s}$ is the surfactant-induced surface tension component. Thus, an increase in concentration of surfactant molecules at the interface between the bubble gas and the surrounding liquid would lead to lower surface tension, which, in turn, would lead to lower internal pressure within the bubble and increased bubble stability against bubble dissolution and recondensation into liquid.

\subsection{Experimental methods}

\subsubsection{Droplet preparation}

PFC droplet emulsions were prepared using tip-sonication approach [28]. Droplet suspensions were prepared by combining water, $5 \% \mathrm{v} / \mathrm{v}$ perfluoropentane (PFP, Fluoromed, Round Rock, TX, USA) and $0.8 \%$ v/v negatively charged fluorinated surfactant Zonyl FSP (Sigma Aldrich, Oakville, ON, Canada). The samples comprised volumes of $10 \mathrm{~mL}$ of the solution, which were first coarse emulsified with a vortex mixer (VM-300, Gemmy Industrial, Cherry Hill, NJ, USA) for $30 \mathrm{~s}$. Following the coarse emulsification, the samples were further emulsified with a tip-sonicator for $60 \mathrm{~s}$ and $80 \%$ duty cycle (Sonifier 250, Branson, Branson, MO, USA) at nominal power output of $100 \mathrm{~W}$. 
Sizing of the droplets was performed using a Zetasizer nano-sizing system (Malvern, Worcestershire, UK).

\subsubsection{Estimation of PFC droplet concentration}

The concentration of PFC droplets under investigation can be estimated using the droplet size distribution and the total volume of PFC used for the emulsion. The total volume of the droplets in the sample can be expressed as:

$$
V_{P F C}=N \int_{0}^{\infty} \frac{4}{3} \pi R_{d}^{3} k\left(R_{d}\right) d R_{d}
$$

where $V_{P F C}$ is the total volume of PFC used in the emulsion, $N$ is the total number of droplets in the solution and $k\left(R_{d}\right)$ is the fraction of the droplets in the solution of radius $R_{d}$. Rearranging the terms in Eq. 3.7 gives:

$$
N=\frac{3 V_{P F C}}{4 \pi \int_{0}^{\infty} R_{d}{ }^{3} k\left(R_{d}\right) d R_{d}}
$$

so that the total amount of droplets can be calculated from the overall PFC volume and droplet size distribution.

\subsubsection{Droplet vaporization experiments}

The schematic of the optical experimental setup used for droplet vaporization experiments is shown in Fig. 3.3. PFC droplets were vaporized by an in-house custom-made PZT5H transducer $(10 \mathrm{MHz}, 1.4 \mathrm{~cm}$ diameter, f/\# 1.0), driven by a Tabor Electronics arbitrary waveform generator (8026, Tabor, Tel Hanan, Israel) amplified by an E\&I 350L power amplifier (E\&I, Rochester, NY, USA). The transducer was focused onto an Opticell containing the droplet sample, diluted 1:2000 in distilled water. The Opticell was placed under a $100 \times$ magnification water-immersion objective $($ N.A. $=1.00)$ of an Olympus BX-FM microscope, coupled to the Brandaris 128 ultra-high speed imaging system [29], providing a diffraction limited spatial resolution of approximately $250 \mathrm{~nm}$ (with a pixel density of $100 \mathrm{~nm} /$ pixel). The field of view in the resulting optical images was $50 \mu \mathrm{m} \times 35 \mu \mathrm{m}$. The focus of the microscope objective was co-aligned with the transducer focus. The setup was placed in a tank of deionized water, kept at a temperature of $37 \pm 2^{\circ} \mathrm{C}$. Droplet samples were vaporized with single ultrasound pulses of $10 \mathrm{MHz}$, from 5 to 10 cycles in duration and at peak negative pressures $\left(\mathrm{P}_{\text {neg }}\right)$ ranging 


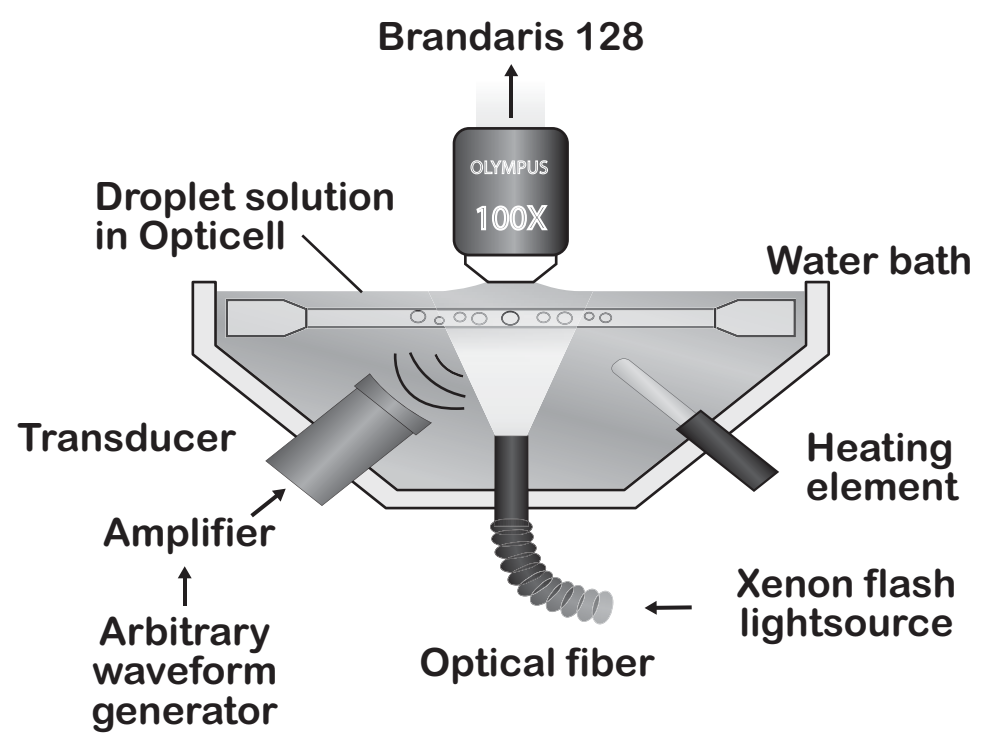

Figure 3.3: Schematic diagram of the experimental setup. Droplet excitation was performed with a single element transducer, while optical detection was done with a $100 \times$ objective of an upright microscope coupled to the Brandaris 128 camera.

from 2.3 to $3.5 \mathrm{MPa}$ (corresponding to Mechanical Index values ranging from 0.73 to 1.11 ). The camera recorded sets of 128 images at either 10 or 15 million frames per second (Mfps), triggered to begin recording at the arrival of the ultrasound pulse, providing temporal resolution from 66 to $100 \mathrm{~ns}$.

\subsubsection{Optical image analysis}

Optical images were analyzed with MATLAB (MathWorks, Natick, MA, USA). Inception or disappearance of the bubble was accompanied by a sharp change in optical image contrast (droplets were either invisible or transparent, while bubbles appeared black). Bubble contours in the optical image frames were traced using a minimum cost algorithm [30]. The bubble radius was calculated as the mean distance from the bubble center of mass to the edge of the bubble in the radial coordinate system. The analysis provided measurements of the bubble radius for spherical bubbles and an average radius estimate for bubbles with a non-spherical shape. The final bubble size was measured either at $10 \mu \mathrm{s}$ after the arrival of the ultrasound pulse, or at the last frame before optical contrast loss in the case of unstable bubbles (the time of 
$t=0$ corresponded to the arrival of the ultrasound pulse). The times for bubble inception and disappearance were also recorded separately. We defined bubbles as stable if they did not disappear within the first $10 \mu$ s following vaporization.

Upon vaporization, a fraction of the bubbles did not appear to be completely in the focus of the microscope, such that, while still visible, their size could not be resolved with confidence. Bubble size measurements were performed for bubbles that remained in the focus of the microscope objective throughout the image sequence. Only the times of bubble presence, rather than their particular sizes, were recorded for bubbles that were not in focus (approximately $25 \%$ of the total number of bubbles). In total, 117 bubbles (in 48 separate recordings) spanning the range of vaporization conditions were examined. In case of bubble coalescence, the final coalesced bubble was considered for the total bubble count and size measurement, rather than the individual bubble precursors coalescing together.

\subsection{Results}

\subsubsection{Droplet sizing and droplet concentration}

A typical droplet size distribution is shown in Fig. 3.4. The droplets were polydisperse in size, ranging from $100 \mathrm{~nm}$ to $1 \mu \mathrm{m}$ in diameter with mean diameter of approximately $400 \mathrm{~nm}$. However, due to the small size of the droplets, it was not possible to measure the size of the individual droplets before vaporization. Furthermore, the light scattering nano-sizing system is unable to measure droplet concentration. As a result, an order of magnitude droplet concentration was estimated using Eq. 3.8 and the measured droplet size distributions (such as that shown in Fig. 3.4). Droplet concentration was estimated to be at 1012 droplets $/ \mathrm{mL}$ for undiluted sample or $5 \cdot 10^{8}$ droplets/mL for the 1:2000 diluted solution. In our experiments, the axial optical field of view was on the order of $10 \mu \mathrm{m}$ (to account for both in focus and out of focus droplets the depth of field of the objective used was on the order of $1 \mu \mathrm{m}$ ), with the field of view at $50 \mu \mathrm{m}$ x $35 \mu \mathrm{m}$, the optically imaged volume was at most $2 \cdot 10^{8} \mathrm{~mL}$. Hence, it is estimated that on average there were at most 10 droplets within the field of view in each image. It should be noted that this is likely to be an overestimation, since the real axial field of view of the objective is smaller, and not all of PFC was incorporated into the droplet solution. However, this approach provides an upper limit order of 


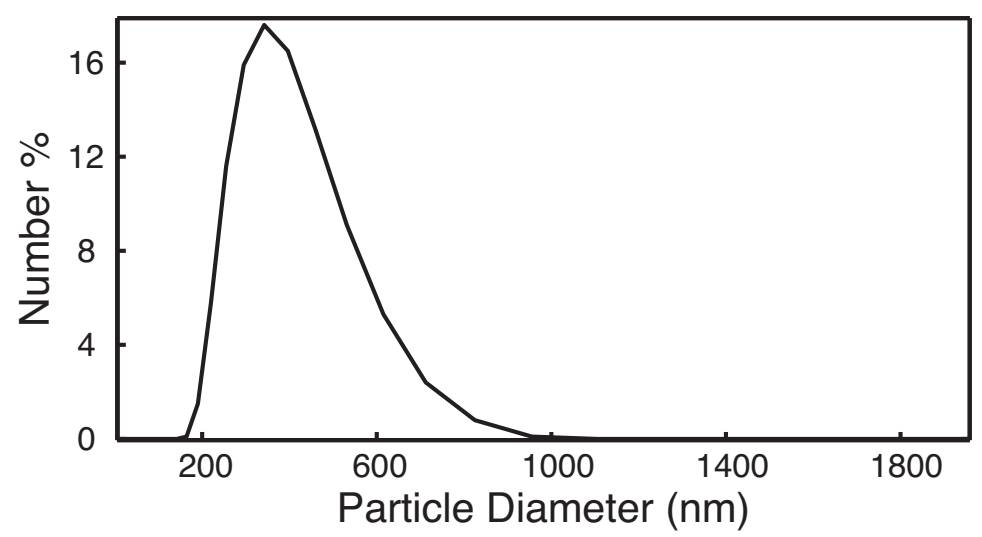

Figure 3.4: Typical droplet size distribution, as measured with Dynamic Light Scattering (DLS). The droplet mean diameter was measured to be at approximately 400 $n m$.

magnitude estimate on the amount of droplets in the optical field of view.

\subsubsection{Vaporization experiments}

Submicron droplets of liquid PFC were successfully vaporized with single pulses of ultrasound, 5 to 10 cycles in duration, at $P_{\text {neg }}$ ranging from 2.3 to 3.5 $\mathrm{MPa}$ (corresponding to an MI range of 0.73 to 1.11). Exposure of the diluted droplet samples (1:2000 dilution) resulted in only individual droplet vaporizations (1-3 vaporization events) in the optical field of view per excitation pulse. An example of a series of high-speed images of droplet vaporization with a 10-cycle pulse (ultrasound burst duration of $1 \mu \mathrm{s}$ ) at $P_{\text {neg }}$ of 2.9 $\mathrm{MPa}$ is shown in Fig. 3.5a, taken at $10 \mathrm{Mfps}$. The corresponding radius-time (R-t) curve obtained from the bubble trace is shown in Fig. 3.5b. The figures show that, following vaporization, the newly created bubble undergoes rapid expansion, followed by rapid shrinkage. After this transient oscillation, the bubble shrinks to a radius of less than $1 \mu \mathrm{m}$, and disappears below the optical resolution limit at approximately $4.5 \mu \mathrm{s}$.

From the investigated bubbles, it was observed that following initial droplet nucleation, the newly created bubble behavior may follow a number of patterns. A diagram of the observed patterns is shown in Fig. 3.6. Following droplet vaporization, after the US vaporization pulse has passed (stage a), the newly created bubbles undergo a growth phase followed by rapid shrinkage 

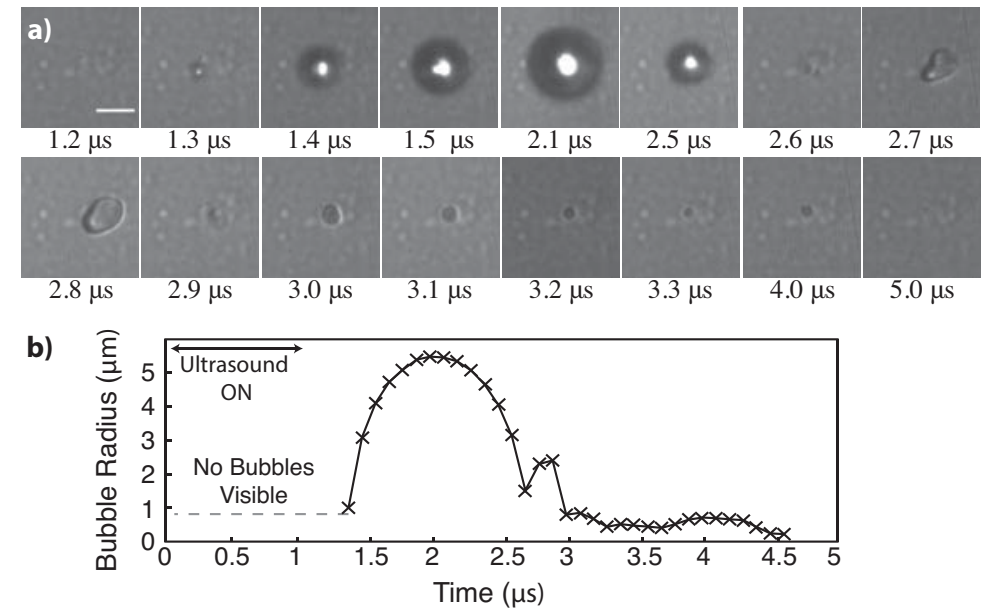

Figure 3.5: Example of a vaporization of a single PFC droplet by a 10-cycle ultrasound pulse at $10 \mathrm{MHz}$ (total pulse length of $1 \mu \mathrm{s}$ ) and $\mathrm{P}_{\text {neg }}$ of $2.9 \mathrm{MPa}$. Images were taken at 10 million frames per second. Time $t=0$ corresponds to the moment of arrival of the excitation ultrasound pulse (a) recorded image sequence. The images depict the initial growth and subsequent collapse and disappearance of the vaporized droplet. Scale bar - $5 \mu \mathrm{m}$ (b) plot of the bubble radius as a function of time. 


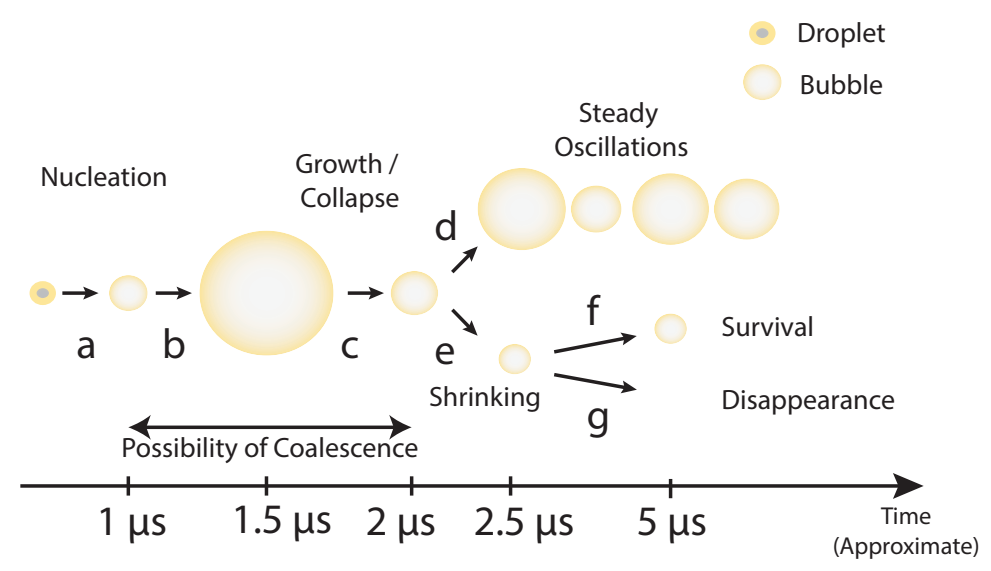

Figure 3.6: Bubble evolution stages after initial vaporization. (a) initial nucleation of the droplet, (b) rapid growth of the newly created bubble to a maximum size, (c) rapid collapse of the bubble after growth, (d) stable oscillation of the bubble for a few cycles with gradual decay, (e) sudden shrinking of the bubble to approximately $1 \mu \mathrm{m}$ in diameter, $(f)$ survival of the small bubble, $(g)$ loss of optical contrast and bubble disappearance. Coalescence of part of the bubbles in close proximity to each other was observed in stages $b$ and $c$. The shown time-scale of the events is approximate, varying from bubble to bubble.

or collapse (stages b and c). At this point, the bubbles can either undergo stable oscillations for a number of cycles eventually coming to rest (stage d), or undergo sudden shrinkage to approximately $1 \mu \mathrm{m}$ in diameter (stage e). The shrunken bubbles could then either remain in their shrunken form (stage f) or disappear from the image completely (stage g). We term stage c as the collapse stage due to the rapid shrinkage of the bubble, and in order to differentiate it from the subsequent shrinking observed in the case of stage e. However, it should be noted that even at the highest frame rates of the camera, it was not possible to tell if the bubble collapse at stage c exhibited the characteristics of a collapsing gas cavity, such as in the case of transient cavitation.

During the initial growth and collapse stage (Fig. 3.6, stages b and c) the bubbles may undergo fragmentation (Fig. 3.7a) and coalescence (Fig. 3.7b). Coalescence was observed in $49 \%$ of the analyzed bubbles, while fragmentation was observed in $17 \%$ of the cases. As might be expected, coalescence of bubbles occurred only when two or more bubbles came into a direct contact either during initial bubble growth or the subsequent collapse (stages b or 

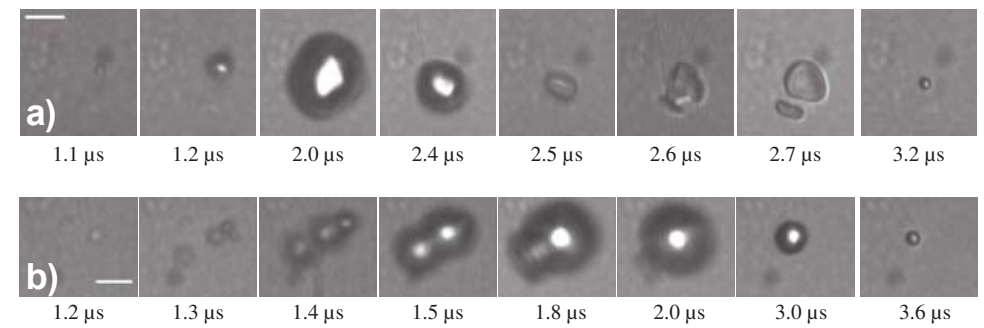

c)

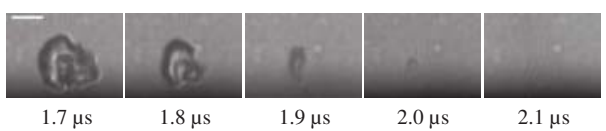

Figure 3.7: Different bubble behaviors during and after vaporization. (a) Droplet vaporization with subsequent fragmentation during the bubble collapse stage (stage c). (b) Example of multiple droplet coalescence. The images depict 4 droplets vaporizing in near proximity to each other. Upon physical contact, the vaporized droplets coalesce, followed by the collapse of the single combined bubble. (c) Rapid disappearance of a bubble following the collapse stage (stage c). Scale bar - $5 \mu \mathrm{m}$.

c). Bubble fragmentation was observed to occur only during the initial collapse phase (stage c). Neither one of these phenomena were observed at any stages subsequent to the collapse. In addition, some cavities were observed to rapidly disappear following the initial collapse, as shown in Fig. 3.7c. However, these cavities did not appear to resemble well-formed bubbles, and due to the rapidity of the events and the irregularities of the bubble shape, these bubbles were not considered in our analysis (the 117 analyzed bubbles did not include the rapidly disappearing ones).

\subsubsection{Post vaporization bubble size}

The bubble size distribution after the initial post-vaporization oscillation (stages $\mathrm{d}$ or e) is shown in Fig. 3.8. For the unstable bubbles, the mean diameter the standard deviation of the bubbles prior to their disappearance was measured to be $1.1 \pm 0.3 \mu \mathrm{m}$. The mean diameter of the bubbles that remained visible after the initial post-nucleation oscillation was measured to be $1.8 \pm 1.5 \mu \mathrm{m}$ at the end of the imaging sequence, at approximately $10 \mu \mathrm{s}$. The two mean sizes are significantly different $(p<0.01)$. However, from the observed size distribution in Fig. 3.8, it is apparent that except for a small number of remaining bubbles at radii above $1 \mu \mathrm{m}$, bubble sizes for both populations are similar. 


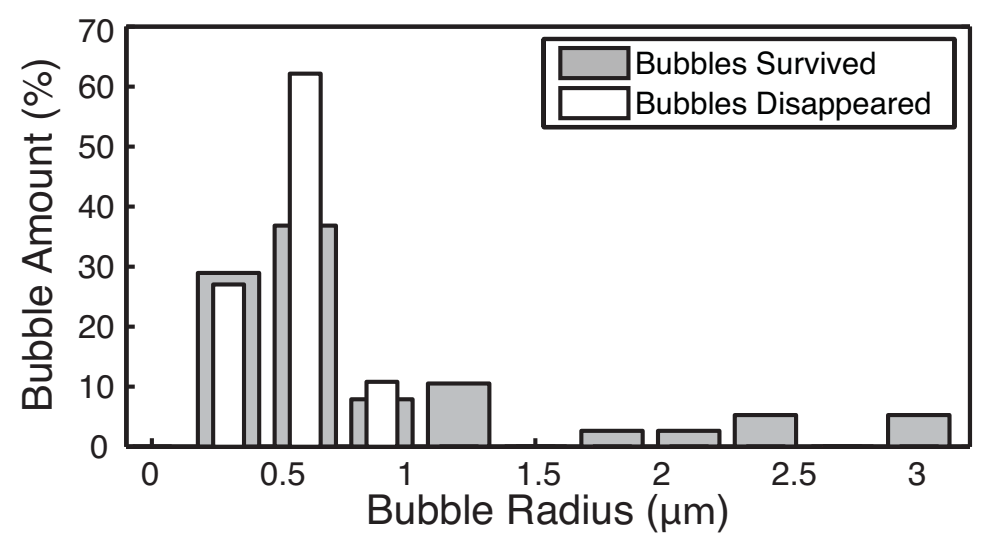

Figure 3.8: Final bubble size distribution for both surviving and disappearing bubbles, after the initial post-vaporization oscillation. None of the bubbles above radius of $1 \mu \mathrm{m}$ were observed to disappear.

\subsubsection{Bubble survival}

Of the 117 analyzed bubbles, 53 remained visible in bubble form (stage d or f in Fig. 3.5), while 64 disappeared (stage g), as observed at the end of the imaging sequence, at approximately $10 \mu$ s after the beginning of the US excitation pulse. There was a strong dependence of probability of bubble survival on coalescence following vaporization and the excitation pressure used to vaporize the droplets. Fig. 3.9 shows a comparison of the ratios of bubble survival for coalesced and non-coalesced bubbles at $P_{\text {neg }}$ of 2.3, 2.6 and 3.2 $\mathrm{MPa}$. The figure shows an increase in the probability of bubble survival when the bubbles undergo coalescence and an inverse dependence of bubble survival on excitation pressure. There was no observed dependence of bubble coalescence rate on ultrasound excitation pressure. Bubble survival rates as a function of size, separately for coalesced and non-coalesced bubbles, are shown in Fig. 3.10. From the figure, it is apparent that for similarly sized bubbles (in separate $250 \mathrm{~nm}$ radius intervals) the rate of survival is also significantly higher for the coalesced bubbles than the non-coalesced ones.

There was no apparent dependence of bubble fragmentation on the rate of bubble survival. Out of the 18 observed fragmented bubbles, 9 eventually disappeared and 9 survived (ratio of 1.0). For the 86 non-fragmented bubbles, 44 disappeared, while 42 survived (ratio of 0.95). For the remaining 13 bubbles, it was unclear whether fragmentation occurred during the collapse or not. 


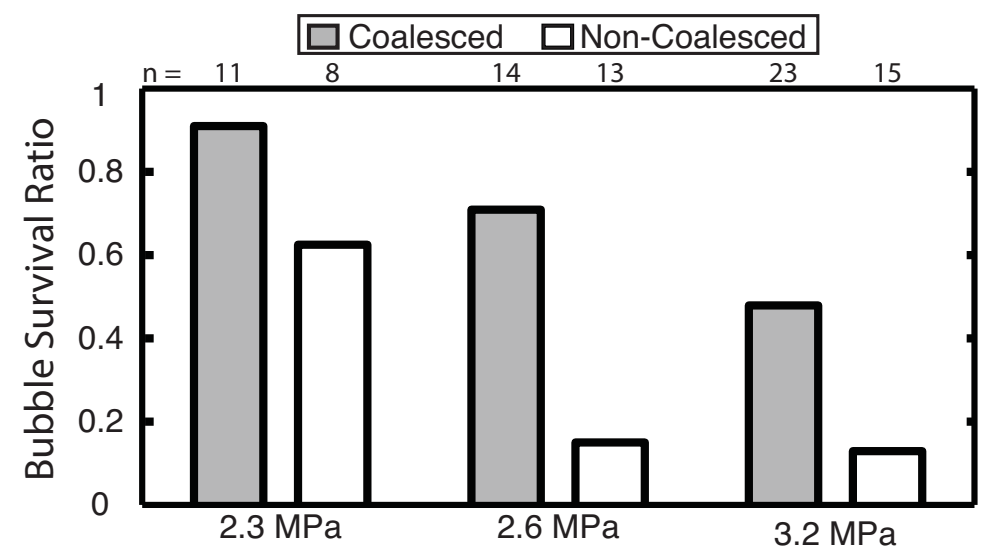

Figure 3.9: Percentage of survived bubbles as a function of ultrasound excitation pressure for coalesced and non-coalesced bubbles. Bubble coalescence and lower excitation pressures lead to higher chance of bubble survival post-vaporization. $\mathrm{n}$ total number of bubbles analyzed for each population.

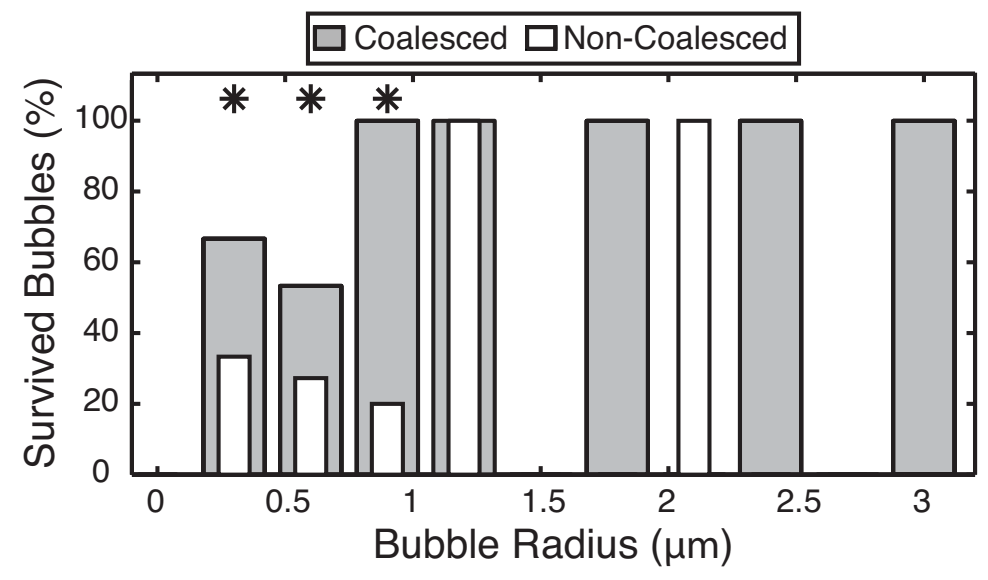

Figure 3.10: Percent of bubbles survived as a function of size, for either coalesced or non-coalesced cases. While 100\% survival was observed for both coalesced and non-coalesced bubbles with radius above $1 \mu \mathrm{m}$, the smaller bubbles that underwent coalescence during vaporization exhibited significantly higher probability of survival than the non-coalesced ones across the entire size range. Stars represent sizes for which the bubble survival rates are significantly different $(\mathrm{p}<0.05)$ as verified by $\chi^{2}$ test. 


\subsection{Discussion}

Unlike previously reported high-speed optical work [19-21], where gradual vaporization of PFP droplets with sizes ranging from 5 to $30 \mu \mathrm{m}$ in diameter was examined on microsecond timescales, in this study we focus on the behavior and stability of the submicron droplets after vaporization, when the ultrasound pulse has passed, as well as on the efficiency of droplet conversion into microbubbles. Vaporization of submicron droplets examined here revealed a number of effects not observed for their micron sized counterparts, as will be discussed in this section.

\subsubsection{Efficiency of droplet vaporization with applied ultra- sound}

Droplet vaporization efficiency is of paramount importance for the potential application of these droplets in medical applications. The possibility of low vaporization efficiency, where thousands of droplets are necessary to produce a few bubbles, may render the application of droplets inefficient, as this would introduce the problem of delivery of such high droplet concentrations to extravasate in tumour regions. It has been previously shown in mice [28] that submicron PFP droplets are retained by tumours and may vaporize to produce detectable bubbles. However, to the best of our knowledge, the direct study of extravasation efficiency of droplets has not yet been performed. Nevertheless, ideally, single droplets should be detectable following US excitation.

In our work, in order to observe individual (13) droplet vaporizations in each image with only occasional coalescence, prepared droplet samples had to be diluted 1:2000 in distilled water. Although it was not possible to directly observe the droplets prior to vaporization due to their small size, we have estimated that for such a dilution, as an order of magnitude upper limit, on average there were at most 10 droplets per image. Hence, a large part (on the order of at least $10 \%$ ) of droplets were vaporized with each ultrasound pulse. Although there are some uncertainties in the estimate of the amount of droplets, such that the exact droplet conversion efficiency is hard to predict, the estimates provide an adequate order of magnitude approximation. The efficiency of initial droplet nucleation due to diagnostic US pulses appears to be quite high, as approximately ten droplets (as opposed to hundreds or thousands) may be sufficient for the initial production of a detectable amount of bubbles, which is of comparable efficiency to the single microbubbles de- 
tectable in an imaging procedure [31]. The final bubble production efficiency, however, is also dependent on bubble survival following vaporization, as will be discussed further in this section.

\subsubsection{Bubble behavior following vaporization}

After initial nucleation, the bubbles were observed to grow to a maximum size (up to $10 \mu \mathrm{m}$ in diameter) and then collapse, decreasing their radius by approximately 5-fold relative to the maximum size attained. This growth and collapse occurs after the ultrasound excitation pulse has passed, and thus is dependent only on the inertia of the surrounding liquid and internal pressure within the bubble, attained during vaporization. A number of cavities (such as the one shown in Fig. 3.7c) were observed to rapidly disappear, without forming a bubble-resembling sphere. While such a transient behavior may be the reason for the previously observed wideband acoustic emissions [32], in this initial study we focus on the stability and recondensation of the well formed bubbles (after stage $\mathrm{c}$ in Fig. 3.6). As a result, we did not consider these transient cavitation events in the analysis for this work.

Following the oscillations, bubbles stabilize in size. With our high speed imaging setup, it was possible to observe the droplets only within the $10 \mu \mathrm{s}$ timeframe. Although we defined stable bubbles as the ones that did not disappear within this timeframe, $10 \mu$ s should not be confused with stability relevant to medical imaging application (of seconds to minutes). In this case the measurement of stability was used to differentiate between recondensing and non-recondensing bubbles. Previous studies (e.g. [14]) have demonstrated bubble stability on millisecond timescales. While, for completeness, it still remains to be shown directly that the bubbles examined here are also stable over hundreds of microseconds, the results of this study may suggest that the stable bubbles observed here in fact survive for time periods much longer than single microseconds without dissolution or recondensation. Bubble dissolution due to gas diffusion on sub-millisecond timescales is not expected to occur, as the bubbles were shown to be stable on milliseconds and seconds time intervals $[14,15]$. Furthermore, even in the case of uncoated PFP bubbles, dissolution would occur at least on a millisecond timescale [33]. Bubble stability against recondensation is not significantly affected by the observation time, at least within the microseconds to seconds time ranges, as shown in Fig. 3.2. This suggests that if a bubble remains stable against recondensation within the first $10 \mu \mathrm{s}$, recondensation will be also unlikely within the first 
$1 \mathrm{~s}$. In addition, the final diameter of the bubbles at approximately $10 \mu \mathrm{s}$ after vaporization was $1.8 \pm 1.5 \mu \mathrm{m}$ and is similar to the value observed by Reznik et al. [14] of $1.4 \pm 1.1 \mu \mathrm{m}$, for $t=1 \mathrm{~ms}$ after vaporization for similarly sized initial droplets. Vaporized droplets observed by Reznik et al. [14] were shown to be stable for at least $1 \mathrm{~s}$ following vaporization, enabling detection by contrast specific ultrasound techniques. This similarity of bubble sizes, droplet preparation protocol and droplet excitation conditions in the current and the previous study, may also suggest that the droplets that survive in the initial microseconds after vaporization are stable enough to persist for at least $1 \mathrm{~s}$ after vaporization and have the potential to serve as useful ultrasound contrast agents.

\subsubsection{Bubble survival post vaporization}

It was observed that following their initial oscillations there is a significant probability of the bubbles losing all optical contrast, effectively vanishing from the image. Loss of contrast due to motion out of focal plane of the microscope objective is unlikely, as no defocusing blur of the bubbles was observed prior to their disappearance. Bubble dissolution as a result of gas diffusion from the bubble is also unlikely on the microsecond timescale. Even in the case of uncoated bubbles, diffusion of PFC for a $1 \mu \mathrm{m}$ bubble would be, at the least, on the order of $1 \mathrm{~ms}$ [34], while the given bubble disappeared over a course of a few frames (hundreds of nanoseconds).

Bubble disappearance over such a short time period is most plausibly attributed to recondensation of the bubble to its initial liquid state. Recondensation of previously vaporized droplets has been proposed previously [8] in an effort to explain some of the observed acoustic phenomena of the vaporized droplets. As shown in Fig. 3.2, the pressure necessary to recondense a PFP microbubble into the liquid droplet form, of approximately $280 \mathrm{kPa}$, is substantially higher than the actual vapor pressure of PFP at $37^{\circ} \mathrm{C}(135 \mathrm{kPa}$, as shown in Fig. 3.1). At the same time, small bubbles experience an increase in Laplace pressure. As an example, the additional Laplace pressure for an uncoated bubble of $500 \mathrm{~nm}$ in radius in water is approximately $300 \mathrm{kPa}$. Thus, the total pressure inside the bubble, assuming hydrostatic pressure of $100 \mathrm{kPa}$, is approximately $400 \mathrm{kPa}$ (as shown in Fig. 3.2), which is sufficiently high to recondense the bubble back into the liquid droplet form. Condensation of small bubbles due to an increase in Laplace pressure has also been suggested previously as the ultimate fate of a dissolving bubble [34]. 
Bigger bubbles experience lower Laplace pressure that reduces the possibility of a condensation event, or eliminates recondensation possibility completely for big enough bubbles. It was experimentally observed (Fig. 3.8) that bubbles above the radius of approximately $\mathrm{R}=1 \mu \mathrm{m}$ did not recondense. This size is in concordance with the threshold bubble size obtained theoretically using the Homogeneous Nucleation Theory of $R_{\text {stable }}=800 \mathrm{~nm}$ for uncoated bubbles. As uncoated bubbles experience the highest Laplace pressure effects, this suggests that PFP bubbles bigger than $1 \mu \mathrm{m}$ in radius, whether encapsulated by a shell material or not, are stable against recondensation for at least $1 \mathrm{~s}$. Due to the large bubble sizes, previous high-speed optical imaging studies of vaporization of micron-sized droplets [19-21] have not observed recondensation behavior.

At the same time, it was shown that not all the bubbles with $\mathrm{R}_{i}$ Rstable recondense, suggesting that bubble size is not the only factor affecting the probability of bubble survival. It was recently shown, both indirectly [35] and directly [25], that droplets retain their initial shell material following vaporization, to produce coated bubbles. Presence of a shell reduces the effective surface tension on the bubble, in turn, reducing the experienced Laplace pressure. As shown by Eq. 3.6, the presence of surfactant molecules on the interface between the bubble and the surrounding liquid can either reduce or completely eliminate the experienced surface tension. It should be noted that variability in the concentration of surfactant shell material on individual vaporized droplets is expected, as such a phenomenon was observed even for the commercially available microbubbles [36]. This variability in the amount of the shell material on the individual bubbles can explain the difference in their fate for bubbles of similar size bubbles with higher concentration of surfactant coating are more likely to avoid condensation than those with less surfactant.

As shown in Fig. 3.9, there is a reduced chance of bubble disappearance after vaporization, if bubbles coalesce either during vaporization or in the oscillation stage, which follows immediately after. Bubble coalescence during vaporization has also been observed previously for micron-sized droplets [21]. Coalescence would increase the total volume and size of the bubble, reducing the effect of Laplace pressure. However, for similar bubble sizes, it was shown that the bubbles that underwent coalescence had a higher chance of survival (Fig. 3.10), indicative of other factors, in addition to size increase, such as increase in surfactant coating concentration. Under ideal conditions, as a result of bubble coalescence, the total amount of surfactant material of the two 
bubbles would combine, as well as the bubbles' volumes. Consequently, coalescence of two bubbles may work to increase the surfactant concentration on the final combined bubble. To illustrate this, consider a simplified situation of two identical bubbles, of radius $R_{0}$ and surfactant concentration $\Gamma_{0}$. The total amount of surfactant on each bubble is then View the MathML source $\mathrm{S}_{0}=4 \pi \mathrm{R}_{0}^{2} \Gamma_{0}$. The new combined bubble would have a radius of View the MathML source $R_{1}=\sqrt[3]{2} R_{0}$. The new surfactant concentration on the bubble is then:

$$
\Gamma_{1}=\frac{2 S_{0}}{4 \pi R_{1}^{2}}=\frac{8 \pi R_{0}^{2} \Gamma_{0}}{4 \pi\left(\sqrt[3]{2} R_{0}\right)^{2}}=\sqrt[3]{2} \Gamma_{0} .
$$

From Eqs. (6) and (9), it follows then that the surfactant ability to compensate surface tension is $\sigma_{s 1}=2 \sigma_{s 0}$, hence the stabilizing ability of the surfactant, against potential recondensation of the bubble, is increased 2-fold. It should be noted, however, that for a well-encapsulated bubble, coalescence with a poorly stabilized bubble may reduce the final encapsulation efficiency for example, following the same considerations as for Eq. 3.9, coalescence of a bubble with surfactant concentration $\Gamma_{0}$ and a bubble of the same size with surfactant concentration of less than $0.26 \Gamma_{0}$ would lead to a final surfactant concentration of $\Gamma_{1}<\Gamma_{0}$. Furthermore, this analysis does not account for the possible loss of surfactant molecules to the bulk of the liquid surrounding the bubble [37] - as a result of coalescence, a portion of the surfactant molecules may dissolve in the surrounding water in an effort to preserve the surface energy [38]. Further studies are necessary to quantify the concentration of surfactant at the droplet surface before and after vaporization and the change in concentration as a result of bubble coalescence.

It should be further noted that the effects of bubble coalescence on bubble stability also depend on the nature of the encapsulating material. A variety of shell materials in addition to negatively charged fluorosurfactants are currently used for droplet preparation, ranging from nonionic fluorosurfactants [28] to phospholipids [7, 26, 39], albumin [25, 40] and polymers [41]. As a result, bubble stability properties and the effects of bubble coalescence on bubble stability may be different for these different droplet formulations, and should be investigated separately.

The presented observations show that maximizing the probability of droplet coalescence either during or immediately following vaporization is advantageous to create stable microbubbles suitable for ultrasound imaging. Since the chance of coalescence is dependent on bubble proximity to each other, it is 
advantageous to increase the initial droplet concentration prior to ultrasoundinduced vaporization to reduce the inter-droplet separation. It was previously observed [14] that there is a decrease in excitation pressure threshold for the inception of stable bubbles with increasing initial droplet concentration. While the proposed explanation for this phenomenon was that an increase in PFC volume results in higher probability of PFC nucleation, it is apparent that stabilization by coalescence is another mechanism for increased efficiency of production of stable bubbles.

Increase in the excitation pressure above the vaporization threshold negatively affects the chances of bubble survival in the first microseconds after vaporization (Fig. 3.9). Previous studies [2,14] have shown an increase in echogenicity from droplet populations with increasing excitation pressure, above the vaporization threshold, which may seem to contradict the current findings. However, the total amount of stable bubbles created is dependent on two factors rate of initial nucleation and vaporization, and subsequent bubble survival. An increase in $P_{\text {neg }}$ leads to a higher rate of nucleation [22] as less energy input is necessary for cavity formation. Hence, an increase in excitation pressure has a positive effect on initial droplet nucleation rate and a negative effect on bubble stability. From the previously published results (e.g. $[2,14])$, it appears that the net effect still results in a higher number of stable bubbles produced.

The results of this study show that stable bubble production efficiency is dependent on the initial nucleation of the droplet and the subsequent bubble survival in the first microseconds following vaporization, which, in turn, is affected by bubble coalescence and bubble shell properties. Although the efficiency of droplet nucleation is relatively high (on the order of at least $10 \%$ ), with unfavourable conditions, up to $90 \%$ of the newly formed bubbles may recondense back into the liquid, resulting in the efficiency of stable bubble generation dropping to around, or even below, $1 \%$.

\subsubsection{Limitation in resolution of initial droplet nucleation}

At $37^{\circ} \mathrm{C}$, the PFP vapor pressure is approximately $135 \mathrm{kPa}$. The critical radius of the nucleation cavity within the superheated PFP at ultrasound $P_{\text {neg }}$ of 2.3 MPa is calculated (Eq. 3.1) to be at $r_{c v}=8 \mathrm{~nm}$. The initial nucleation cavity is much smaller than the droplet size, which is on the order of $100 \mathrm{~nm}$, and may form within the droplet. Such nucleation behavior was observed earlier in the case of the bigger, micron-sized droplets [19,21]. The initial nucleation 
cavity may vaporize the droplet completely before the event is observed with our optical system, due to its impressive but still limited temporal and spatial resolution.

It is apparent (e.g. from Fig. 3.5b) that there is a delay between the arrival and passing of the ultrasound pulse and inception of a detectable bubble. Although in this work we focus on examination of the bubble product after vaporization, and the study of initial nucleation of the droplet under ultrasound is beyond the scope of this paper, we may speculate on the possible reasons for such an observed delay. Due to the imaging frame rate of 10 Mfps, which is low relative to the $10 \mathrm{MHz}$ ultrasound excitation frequency, each image in the sequence captures a single cycle of ultrasound. Therefore, there is a possibility that transient behavior, occurring only during the rarefactional part of the ultrasound, wave may be missed on our images. A bubble, nucleated while the ultrasound pulse is on, is easily disrupted when exposed to the high compressional pressures due to transient cavitation or recondensation: a gas bubble close to the resonant size associated with the excitation frequency (approximately $1 \mu \mathrm{m}$ in diameter) at an MI of around 1.0, as was used in our experiments, may undergo transient cavitation and collapse; the high compressional pressures may cause the bubble to quickly condense back into the liquid droplet form. Recently, it has been shown [39] that exposure of microbubbles to high static compressional pressures may be utilized as a method of production of submicron PFC droplets. In such a scenario, a bubble may be both nucleated and recondensed (or cavitated) within a single cycle of ultrasound, with the process evading observation by our imaging setup. Future studies, utilizing lower excitation frequencies and higher frame rates will investigate the process of initial nucleation and droplet behavior during US excitation in more detail.

It should be noted that the excitation frequencies used here are at the higher part of the clinical diagnostic imaging frequency range. In a clinical setting, due to frequency dependent attenuation, it may be difficult to achieve pressures as high as 2 to $3 \mathrm{MPa}$, necessary for vaporization, deep within the body at a frequency of $10 \mathrm{MHz}$. However, the use of such frequencies should still be adequate to relatively superficial targets, such as the breast. 


\subsection{Summary}

Submicron PFC droplet vaporization was observed for the first time on the nanosecond scale. It was estimated that the efficiency of conversion of droplets into bubbles when exposed to an acoustic pulse with $P_{\text {neg }}$ within the diagnostic range is of the order of magnitude of at least $10 \%$. The behavior of vaporized submicron droplets immediately following vaporization is different to their micron-sized counterparts. Following vaporization and transient oscillations, bubbles may remain in the micron-sized form, shrink to approximately $1 \mu \mathrm{m}$ in diameter or condense back into their liquid form. Furthermore, droplets may undergo coalescence or fragmentation during the initial oscillations following droplet vaporization. Depending on the vaporization conditions, it is estimated that, up to $90 \%$ of the initial vaporized droplets may recondense back into the liquid form within several microseconds following vaporization.

Presence of coating material on the bubble surface affects bubble stability and probability of bubble survival for adequately long time periods to create acoustically active particles for diagnostic ultrasound contrast imaging. Bubble coalescence, effectively increasing the shell material concentration on the bubble surface, increases the chances of bubble survival. An increase in initial droplet concentration, leading to reduced inter-droplet separation distances, hence, is beneficial for the production of stable microbubbles.

The results presented in this study suggest a number of conditions affecting the probability of generation of stable microbubbles from PFC droplets, which have not been reported to date. The study shows that although initial nucleation of droplets is necessary for the production of echogenic bubbles, additional factors, such as bubble coalescence and bubble shell properties, significantly affect the probability of inception of bubbles that are stable. Hence, the efficiency of initial droplet vaporization, and the efficiency of stable bubble production may be very different, depending on the excitation conditions. These conditions should be considered for production of the convertible PFC droplets that can serve as ultrasound contrast agents.

\section{References}

[1] T. Matsunaga, P. Sheeran, S. Luois, J. Streeter, L. Mullin, B. Banerjee, and P. Dayton, "Phase-change nanoparticles using highly volatile 
per-fluorocarbons: Toward a platform for extravascular ultrasound imaging", Theranostics 2, 1185-1198 (2012).

[2] O. Kripfgans, J. Fowlkes, D. Miller, O. Eldevik, and P. Carson, "Acoustic droplet vaporization for therapeutic and diagnostic applications", Ultrasound Med. Biol. 114, 1177-1189 (2000).

[3] P. Sheeran and P. Dayton, "Phase-change contrast agents for imaging and therapy”, Curr. Pharm. Des. 18, 2152-2165 (2013).

[4] M. Zhang, M. Fabiilli, K. Haworth, J. Fowlkes, O. Kripfgans, W. Roberts, K. Ives, and P. Carson, "Initial investigation of acoustic droplet vaporization for occlusion in canine kidney", Ultrasound Med. Biol. 36, 1691-1703 (2010).

[5] C. Carneal, O. Kripfgans, J. Krucker, P. Carson, and J. Fowlkes, “A tissue-mimicking ultrasound test object using droplet vaporization to create point targets", IEEE Trans. Ultrason. Ferr. 58, 2013-2025 (2011).

[6] P. Zhang and T. Porter, "An in vitro study of a phase-shift nanoemulsion: a potential nucleation agent for bubble-enhanced hifu tumor ablation", Ultrasound Med. Biol. 36, 1856-1866 (2010).

[7] K. Schad and K. Hynynen, "In vitro characterization of perfluorocarbon droplets for focused ultrasound therapy", Phys. Med. Biol. 55, 49334947 (1999).

[8] K.-I. Kawabata, N. Sugita, H. Yoshikawa, T. Azuma, and S. Umemura, "Nanoparticles with multiple perfluorocarbons for controllable ultrasonically induced phase shifting”, Jpn. J. Appl. Phys. 44, 4548-4552 (2005).

[9] M. Zhang, M. L. Fabiilli, K. J. Haworth, F. Padilla, S. D. Swanson, O. D. Kripfgans, P. Carson, and J. Fowlkes, "Acoustic droplet vaporization for enhancement of thermal ablation by high intensity focused ultrasound", Acad. Radiol. 18, 1123-1132 (2011).

[10] M. Fabiilli, K. Haworth, I. Sebastian, O. Kripfgans, P. Carson, and J. Fowlkes, "Delivery of chlorambucil using an acoustically-triggered perfluoropentane emulsion.", Ultrasound Med. Biol. 36, 1364-1375 (2010). 
[11] M. Fabiilli, J. Lee, O. Kripfgans, P. Carson, and J. Fowlkes, "Delivery of water-soluble drugs using acoustically triggered perfluorocarbon double emulsions", Pharm. Res. 27, 2753-2765 (2010).

[12] N. Rapoport, Z. Gao, and A. Kennedy, "Multifunctional nanoparticles for combining ultrasonic tumor imaging and targeted chemotherapy", J. Natl. Cancer Inst. 99, 492-510 (2007).

[13] O. Couture, A. Urban, A. Bretagne, L. Martinez, M. Tanter, and P. Tabeling, "In vivo targeted delivery of large payloads with an ultrasound clinical scanner", Med. Phys. 39, 5229-5237 (2012).

[14] N. Reznik, R. Williams, and P. Burns, "Investigation of vaporized submicron perfluorocarbon droplets as an ultrasound contrast agent", Ultrasound Med. Biol. 37, 1271-1279 (2011).

[15] P. Sheeran, V. Wong, S. Luois, R. McFarland, W. Ross, S. Feingold, T. Matsunaga, and P. Dayton, "Decafluorobutane as a phase-change contrast agent for low-energy extravascular ultrasonic imaging", Ultrasound Med. Biol. 37, 1518-1530 (2011).

[16] H. Maeda, J. Wu, T. Sawa, Y. Matsumura, and K. Hori, "Tumor vascular permeability and the epr effect in macromolecular therapeutics: A review", J. Control Release 165, 271-284 (2000).

[17] P. Burns and S. Wilson, "Focal liver masses: Enhancement patterns on contrast-enhanced imagesconcordance of us scans with ct scans and $\mathrm{mr}$ images", Radiology 242, 162-174 (2007).

[18] J. Hudson, R. Williams, B. Lloyd, M. Atri, T. Kim, G. Bjarnason, and P. Burns, "Improved flow measurement using microbubble contrast agents and disruption-replenishment: clinical application to tumour monitoring", Ultrasound Med. Biol. 37, 1210-1221 (2011).

[19] O. Kripfgans, M. Fabiilli, P. Carson, and J. Fowlkes, "On the acoustic vaporization of micrometer-sized droplets", J. Acoust. Soc. Am. 116, 272-281 (2004).

[20] Z. Wong, O. Kripfgans, A. Qamar, J. Fowlkes, and J. L. Bull, "Bubble evolution in acoustic droplet vaporization at physiological temperature via ultra-high speed imaging", Soft Matter 7, 4009-4016 (2011). 
[21] K. J. Haworth, O. Kripfgans, and J. L. Bull, "Initial growth and coalescence of acoustically vaporized perfluorocarbon microdroplets", IEEE Trans. Ultrason. Ferroelec. Freq. Contr. (2008).

[22] C. Church, "Spontaneous homogeneous nucleation, inertial cavitation and the safety of diagnostic ultrasound", Ultrasound Med. Biol. 28, 1349-1364 (2003).

[23] E. Barber and G. Cady, "Vapor pressures of perfluoropentanes", J. Phys. Chem. 60, 504-505 (1956).

[24] L. Allen and J. Kassner, "The nucleation of water vapor in the absence of particulate matter and ions", J. Colloid Interf. Sci. 30, 81-93 (1969).

[25] N. Reznik, M. Seo, R. Williams, E. Bolewska-Pedyczak, M. Lee, N. Matsuura, J. Gariepy, F. Foster, and P. Burns, "Optical studies of vaporization and stability of fluorescently labelled perfluorocarbon droplets", Phys. Med. Biol. 57, 7205-7217 (2012).

[26] E. Stride, "The influence of surface adsorption on microbubble dynamics”, Philos. Trans. Roy. Soc. A 366, 2103 (2008).

[27] A. Atchley, "The blake threshold of a cavitation nucleus having a radius-dependent surface tension", J. Acoust. Soc. Am. 114, 1678-1690 (1989).

[28] R. Williams, C. Wright, E. Cherin, N. Reznik, M. Lee, I. Gorelikov, F. Foster, N. Matsuura, and P. Burns, "Characterization of submicron phase-change perfluorocarbon droplets for extravascular ultrasound imaging of cancer", Ultrasound Med. Biol. 39, 475-489 (2013).

[29] C. Chin, C. Lance, J. Borsboom, F. Mastik, M. Frijlink, N. de Jong, M. Versluis, and D. Lohse, "Brandaris 128: A digital 25 million frames per second camera with 128 highly sensitive frames", Rev. Sci. Instrum. 74, 5026-5034 (2003).

[30] S. V. der Meer, B. Dollet, M. Voormolen, C. Chin, A. Bouakaz, N. de Jong, M. Versluis, and D. Lohse, "Microbubble spectroscopy of ultrasound contrast agents", J. Acoust. Soc. Am. 39, 648-656 (2007). 
[31] A. Klibanov, P. Rasche, M. Hughes, J. Wojdyla, K. Galen, J. Wible, and G. Brandenburger, "Detection of individual microbubbles of ultrasound contrast agents: imaging of free-floating and targeted bubbles", Invest. Radiol. 39, 187-195 (2004).

[32] T. Giesecke and K. Hynynen, "Ultrasound-mediated cavitation thresholds of liquid perfluorocarbon droplets in vitro", Ultrasound Med. Biol. 29, 1359-1365 (2003).

[33] K. Sarkar, A. Katiyar, and P. Jain, "Growth and dissolution of an encapsulated contrast microbubble: effects of encapsulation permeability", Ultrasound Med. Biol. 35, 1385-1396 (2009).

[34] A. Kabalnov, D. Klein, T. Pelura, E. Schutt, and J. Weers, "Detection of individual microbubbles of ultrasound contrast agents: imaging of free-floating and targeted bubbles", Ultrasound Med. Biol. 24, 739-749 (1998).

[35] C.-H. Wang, S.-T. Kang, Y.-H. Lee, Y.-L. Luo, Y.-F. Huang, and C.-K. Yeh, "Aptamer-conjugated and drug-loaded acoustic droplets for ultrasound theranosis", Biomaterials 33, 1939-1947 (2012).

[36] M. Overvelde, V. Garbin, J. Sijl, B. Dollet, N. de Jong, D. Lohse, and M. Versluis, "Nonlinear shell behavior of phospholipid-coated microbubbles", Ultrasound Med. Biol. 36, 2080-2092 (2010).

[37] D. Yount, "On the evolution, generation, and regeneration of gas cavitation nuclei", J. Acoust. Soc. Am. 71, 1473-1481 (2011).

[38] D. Evans and H. Wennerstrm, "The colloidal domain: Where physics, chemistry and biology meet", Wiley-VCH, New York (1999).

[39] P. S. Sheeran, S. Luois, P. A. Dayton, and T. O. Matsunaga, "Formulation and acoustic studies of a new phase-shift agent for diagnostic and therapeutic ultrasound", Langmuir 27, 10412-10420 (2011).

[40] M. Fabiilli, K. Haworth, N. Fakhri, O. Kripfgans, P. Carson, and J. Fowlkes, "The role of inertial cavitation in acoustic droplet vaporization.”, IEEE Trans. Ultrason. Ferroelec. Freq. Contr. 56, 1006-1017 (2009). 
[41] N. Rapoport, Z. Gao, and A. Kennedy, "Cavitation properties of block copolymer stabilized phase-shift nanoemulsions used as drug carriers", 36, 419-429 (2010). 


\section{4 \\ Focusing and nucleation}

Acoustically sensitive emulsion droplets composed of a liquid perfluorocarbon have the potential to be a highly efficient system for local drug delivery, embolotherapy or for tumor imaging. The physical mechanisms underlying the acoustic activation of these phase-change emulsions into a bubbly dispersion, termed acoustic droplet vaporisation, have not been well understood. The droplets have a very high activation threshold, its frequency dependence does not comply with homogeneous nucleation theory and localized nucleation spots have been observed. Here we show that acoustic droplet vaporization is initiated by a combination of two phenomena: highly nonlinear distortion of the acoustic wave before it hits the droplet, and focusing of the distorted wave by the droplet itself. At high excitation pressures, nonlinear distortion causes significant superharmonics with wavelengths of the order of the droplet size. These superharmonics strongly contribute to the focusing effect, therefore the proposed mechanism also explains the observed pressure thresholding effect. Our new interpretation is validated with experimental data captured with an ultra high-speed camera on the positions of the nucle-

\footnotetext{
${ }^{\ddagger}$ Published as: Oleksandr Shpak, Martin Verweij, Rik Vos, Nico de Jong, Detlef Lohse, and Michel Versluis, "Acoustic droplet vaporization is initiated by superharmonic focusing", Proceedings of the National Academy of Sciences of the United States of America 111 (5). 1697-1702 (2014).
} 
ation spots, where we find excellent agreement with the theoretical prediction. Moreover, the presented mechanism explains the hitherto counterintuitive dependence of the nucleation threshold on the ultrasound frequency. The new physical insight allows for the optimization of acoustic droplet vaporization for therapeutic applications, in particular with respect to the acoustic pressures required for activation, thereby minimizing the negative bioeffects associated with the use of high-intensity ultrasound.

\subsection{Introduction}

Over the last 15 years, nanomedicine has emerged as a promising field to address current problems of chemotherapy [1-4]. Several drug-carrying constructs have been suggested to decrease the severe side effects of systemic injection on healthy tissue. The common strategy for such a local drug delivery application is the encapsulation of the drugs in polymeric micelles, hollow particles, liposomes or emulsion droplets. The encapsulation allows for targeted and triggered release of the content and the administration of bioactive compounds which have low aqueous solubility [5].

One approach is the use of injectable phase-change emulsion microdroplets composed of a low boiling point perfluorocarbon (PFC), such as perfluoropentane (PFP, $29^{\circ} \mathrm{C}$ boiling point). PFC emulsions have been studied in a wide variety of diagnostic and therapeutic applications such as drug delivery, tumor imaging, and embolotherapy [6]. Ultrasound can be used to induce a phase-transition of such droplets to gas bubbles, a process known as acoustic droplet vaporization (ADV) [7]. Since ultrasound can be applied locally and non-invasively, ADV has been investigated as a means of localized drug delivery, especially for therapeutic agents with a narrow therapeutic index, such as chemotherapeutic drugs [5, 8-10]. The droplets are stabilized by a surfactant shell to prevent their coalescence. The PFP emulsion does not spontaneously vaporize when injected in vivo at $37^{\circ} \mathrm{C}[11,12]$ until the droplets are exposed to ultrasound at sufficiently high pressure amplitude [7]. Recent studies have also demonstrated that PFC nanodroplets (size $\sim 200 \mathrm{~nm}$ ) may extravasate through leaky tumor vasculature, thus passively accumulating in the interstitial space, with the potential to enhance the therapeutic outcome $[13,14]$.

Even though the ADV approach for localized drug delivery shows great promise $[13,15]$, the physical mechanisms underlying the nature of the ADV process have not been well explained. The pressure amplitudes required to 
induce the phase transition of the acoustically sensitive droplets appear to be substantial, with mechanical indices [16] reported to be as high as four [7], well above the FDA-approved limit for diagnostic ultrasound. A pronounced thresholding behaviour has been observed for the activation, meaning that there is negligible probability for detection up to some peak negative pressure amplitude, after which the probability increases linearly with the applied acoustic pressure [7, 17]. Several groups have reported a dependence of the pressure threshold on the driving frequency [7, 17-19]. The required threshold has been found to decrease with increasing frequency, whereas the cavitation threshold in liquids is expected to increase with increasing frequency [16]. There is also an unexplained decrease of the threshold pressure with increasing size of the droplets [20, 21]. Finally, ultra high-speed imaging has allowed for the construction of spatial and temporal nucleation maps [22]. This showed that the nucleation spots inside the droplets were highly localized for some bubbles, whereas other bubbles had nucleation spots at random positions throughout the droplet. The authors suggested that the location of such spots may be a function of the droplet size [22]. The authors also pointed out that temporally the initiation of the nucleation is shifted towards the end of the rarefactional half cycle of the ultrasound pulse.

Here we elucidate the physical mechanism which is responsible for all of the above phenomena. We show that acoustic droplet vaporization is initiated by the focusing of a nonlinear acoustic wave on a specific spot inside the droplet. The focusing results from the spherical shape of the droplet and the acoustic impedance mismatch between the PFC droplet and its exterior. The phenomenon is facilitated by the nonlinear propagation of ultrasound, which builds up superharmonics that are necessary to induce the focusing effect by having a wavelength of the order of the droplet size. Below we will present the theory for this problem. We will explain the approach for analyzing the distortion of the focused ultrasound wave and generation of superharmonics due to nonlinear propagation, and subsequently derive the expression for the diffracted ultrasound inside the droplet. We will then combine theory and numerical computations to quantify the effect of superharmonic focusing within the droplet, which allows for the exploration of the full parameter space of acoustic pressure, frequency, transducer geometry and droplet size. Finally, our theoretical treatment is supported with precise ultra high-speed imaging experiments near the vaporization threshold of single microdroplets, which show the phenomena to be droplet size dependent. 


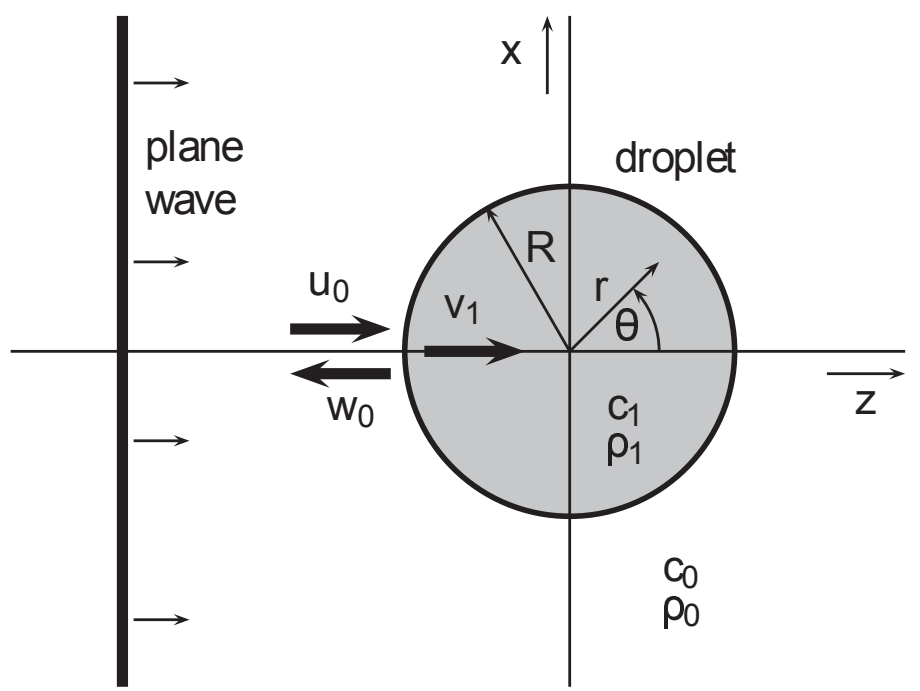

Figure 4.1: Schematic of the diffraction of an acoustic plane wave within a spherical droplet. Incoming plane wave $\mathfrak{u}_{0}$ propagating from left to right, scattered wave $w_{0}$ outside the droplet and refracted wave $v_{1}$ inside the droplet.

\subsection{Theory}

\subsubsection{Nonlinear propagation}

The acoustic pressure amplitudes required to nucleate the droplet have been observed to be very high. In a typical ADV experiment, prior to impinging on the droplet, the ultrasound wave from a focused transducer travels a distance on the order of a few centimeters [7, 17-19, 22-24]. Under these conditions the wave that arrives at the focus of the transducer can be highly distorted due to nonlinear propagation $[25,26]$. The use of a focused transducer permits that the nonlinearly distorted wave can be calculated using the KhokhlovZabolotskaya-Kuznetsov (KZK) equation [27, 28]:

$$
\frac{\partial^{2} p}{\partial z \partial \tau}=\frac{c_{0}}{2} \nabla^{2} p+\frac{\delta}{2 c_{0}^{3}} \frac{\partial^{3} p}{\partial \tau^{3}}+\frac{\beta}{2 \rho_{0} c_{0}^{3}} \frac{\partial^{2} p^{2}}{\partial \tau^{2}},
$$

with $p$ the pressure, $\beta$ the coefficient of nonlinearity of the medium, and $\delta$ its sound diffusivity. The variable $\tau$ denotes that the retarded time $\tau=t-(z-$ $\left.z_{0}\right) / c_{0}$, with $z_{0}$ being the distance between the transducer and the droplet. The derivation of the KZK equation involves a parabolic approximation which is valid for forward propagating waves in a cone with an half-opening angle of 
up to 16 degrees around the transducer axis [29-31]. This requirement is often met for the waves emitted from a regular piston transducer.

All the subsequent derivations are given in the complex representation. The pressure field at the acoustic focus of the transducer obtained from the solution of the KZK-equation can be expanded in a Fourier series:

$$
p_{K Z K}(t)=\sum_{n=0}^{\infty} a_{n} e^{i\left(n \omega t+\phi_{n}\right)},
$$

where $a_{n}$ and $\phi_{n}$ are the amplitudes and the phases of the $n$-th harmonic component of the ultrasound wave. Because nonlinear waveform deformation builds up over distance and the droplet is four orders smaller in size than the distance to the transducer, we disregard the additional nonlinear distortion inside the droplet. This implies that wave propagation inside the droplet is considered linear, so the superposition theorem holds and each harmonic component may be analyzed individually, as we will do in the following.

\subsubsection{Diffraction within a spherical droplet}

Let us consider an incident plane acoustic pressure wave composed of a single Fourier component $p=a e^{i(\omega t-k z+\phi)}$ with pressure amplitude a propagating in the $z$-direction in a medium with speed of sound $c_{0}$, density of mass $\rho_{0}$ and wavenumber (or spatial frequency) $k_{0}=\omega / c_{0}$. It interacts with a spherical droplet with radius $R$, speed of sound $c_{1}$, density of mass $\rho_{1}$ and wavenumber $k_{1}=\omega / c_{1}$. The angular frequency of the ultrasound wave is $\omega=2 \pi f$, with $f$ the frequency of the ultrasound wave.

Let us now find the pressure wave inside the droplet. The time dependence of the refracted pressure waves (the one inside the droplet) and the scattered pressure wave (the one outside the droplet) is determined by the $e^{i \omega t+\phi}$ multiplier. As a consequence, the acoustic wave equation $\nabla^{2} p-\frac{1}{c_{s}^{2}} \frac{\partial^{2} p}{\partial t^{2}}=0$ reduces to the Helmholtz equation [32]

$$
\nabla^{2} v_{s}+k_{s}^{2} v_{s}=0
$$

where $k_{s}=\omega / c_{s}$ is again the wavenumber and $v_{s}$ is the spatial pressure wave (without the $e^{i \omega t+\phi}$ time dependency. $s=0,1$ represents the notation of the different media; 0 for the exterior and 1 for the interior. The wave outside the droplet can then be expressed as $v_{0}=u_{0}+w_{0}$, where $u_{0}$ represents the 
incident plane wave (a known function), and $w_{0}$ the scattered wave, and the wave inside the droplet is indicated by $v_{1}$, see also Fig. 4.1.

At the droplet interface the pressure field must satisfy two boundary conditions:

$$
\begin{gathered}
v_{1}=u_{0}+w_{0}, \\
\frac{1}{\rho_{1}} \frac{\partial v_{1}}{\partial n}=\frac{1}{\rho_{0}} \frac{\partial u_{0}}{\partial n}+\frac{1}{\rho_{0}} \frac{\partial w_{0}}{\partial n},
\end{gathered}
$$

where $\partial / \partial n$ indicates the normal derivative to the droplet interface. The first boundary condition follows from the condition of continuous pressure at the droplet surface, as no force can be exerted on an infinitesimally small boundary. And the second has the physical meaning of continuity of a fluid particle displacement normal to the surface. The incident plane wave can be expanded in spherical coordinates as follows [32]:

$$
u_{0}=e^{-i k_{0} r \cos \theta}=\sum_{m=0}^{\infty}(-i)^{m}(2 m+1) j_{m}\left(k_{0} r\right) P_{m}(\cos \theta),
$$

where $j_{m}\left(k_{0} r\right)=\sqrt{\frac{\pi}{2 k_{0} r}} J_{m+\frac{1}{2}}\left(k_{0} r\right)$, with $J_{m+\frac{1}{2}}\left(k_{0} r\right)$ a Bessel function of the first kind of order $\left(m+\frac{1}{2}\right)$, and $P_{m}(\cos \theta)$ the Legendre polynomial of order $\mathrm{m}$. The symbols $r$ and $\theta$ denote spherical coordinates with the $\theta=0$ direction being aligned along the propagation direction of the incident ultrasound wave $u_{0}$. Based on symmetry considerations both refracted $v_{1}$ and scattered $w_{0}$ waves are axisymmetric functions and must both satisfy the Helmholtz wave equation Eq. (4.3). Let us find such solutions of the unknown functions $v_{1}$ and $w_{0}$ in the form of Eq. (4.6):

$$
\begin{aligned}
v_{1} & =\sum_{m=0}^{\infty} \alpha_{m} j_{m}\left(k_{1} r\right) P_{m}(\cos \theta), \\
w_{0} & =\sum_{m=0}^{\infty} \beta_{m} h_{m}^{(2)}\left(k_{0} r\right) P_{m}(\cos \theta),
\end{aligned}
$$

where $\alpha_{m}, \beta_{m}$ are unknown coefficients, $j_{m}\left(k_{1} r\right)$ as before, and $h_{m}^{(2)}\left(k_{0} r\right)=$ $\sqrt{\frac{\pi}{2 k_{0} r}} H_{m+\frac{1}{2}}^{(2)}\left(k_{0} r\right)$ with $H_{m+\frac{1}{2}}^{(2)}\left(k_{0} r\right)$ the Hankel function of the second kind of order $\left(m+\frac{1}{2}\right)$. Hankel functions of the second kind are chosen as linearly independent basis for the expansion of the scattered wave $w_{0}$, because they 
automatically satisfy the Sommerfeld radiation conditions at infinity. One can find the $\alpha_{m}$ and $\beta_{m}$ coefficients by substituting Eqs. (4.7) and (4.8) at the droplet interface $(r=R)$ into the two boundary conditions, Eqs. (4.4) and (4.5), and using the known function $\mathfrak{u}_{0}$ from Eq. (4.6). Equalizing the prefactors of $P_{m}(\cos \theta)$ (they must be equal, because all $P_{m}(\cos \theta)$ are linearly independent functions of $\cos \theta)$ gives:

$$
\alpha_{m}=\gamma_{m} \frac{j_{m}\left(k_{0} R\right) h_{m}^{\prime(2)}\left(k_{0} R\right)-h_{m}^{(2)}\left(k_{0} R\right) j_{m}^{\prime}\left(k_{0} R\right)}{j_{m}\left(k_{1} R\right) h_{m}^{\prime(2)}\left(k_{0} R\right)-\frac{k_{1}}{k_{0}} \frac{\rho_{o}}{\rho_{1}} h_{m}^{(2)}\left(k_{0} R\right) j_{m}^{\prime}\left(k_{1} R\right)},
$$

where $\gamma_{m}=(-i)^{m}(2 m+1)$, and $j_{m}^{\prime}$ and $h_{m}^{\prime(2)}$ denote the derivatives of the respective functions. The coefficients $\alpha_{m}$ are now known and expressed in terms of the dimensionless parameters $k_{0} R$ and $k_{1} R$. Thus, by reintroducing the exponential time factor that was dropped earlier, the pressure wave inside the droplet at any given spatial point $(r, \theta)$ and any instant of time $t$ can be written as:

$$
p_{\text {inside }}(r, \theta, t)=a e^{i(\omega t+\phi)} \sum_{m=0}^{\infty} \alpha_{m} j_{m}\left(k_{1} r\right) P_{m}(\cos \theta),
$$

where $\phi$ is related to the phase of the incident field and $a$ is the amplitude of the incident pressure wave.

Equation (4.10) was obtained for an incident sinusoidal wave. It can now be extended to the case of a nonlinear pressure waveform. For the incident wave, we superimpose the Fourier components as in Eq. (4.2). With $a_{n}$ and $\phi_{n}$ known, the corresponding Fourier component inside the droplet is found when substituted into Eq. (4.10). Superposition of these internal Fourier components is then used to calculate how the nonlinear pressure wave is diffracted within the small spherical droplet:

$$
p_{\text {inside }}(r, \theta, t)=\sum_{n=0}^{\infty} \sum_{m=0}^{\infty} a_{n} e^{i\left(n \omega t+\phi_{n}\right)} \alpha_{m n} j_{m}\left(k_{1} r\right) P_{m}(\cos \theta),
$$

where $\alpha_{m n}$ is computed by means of Eq. (4.9) with $k_{0}$ and $k_{1}$ being replaced by $n k_{0}$ and $n k_{1}$, respectively.

\subsection{Results and discussions}

The distorted wave is calculated from the real part of Eq. (4.2) and plotted in Fig. 4.2 in spatial coordinates in the propagation direction $z$, along the axis of 


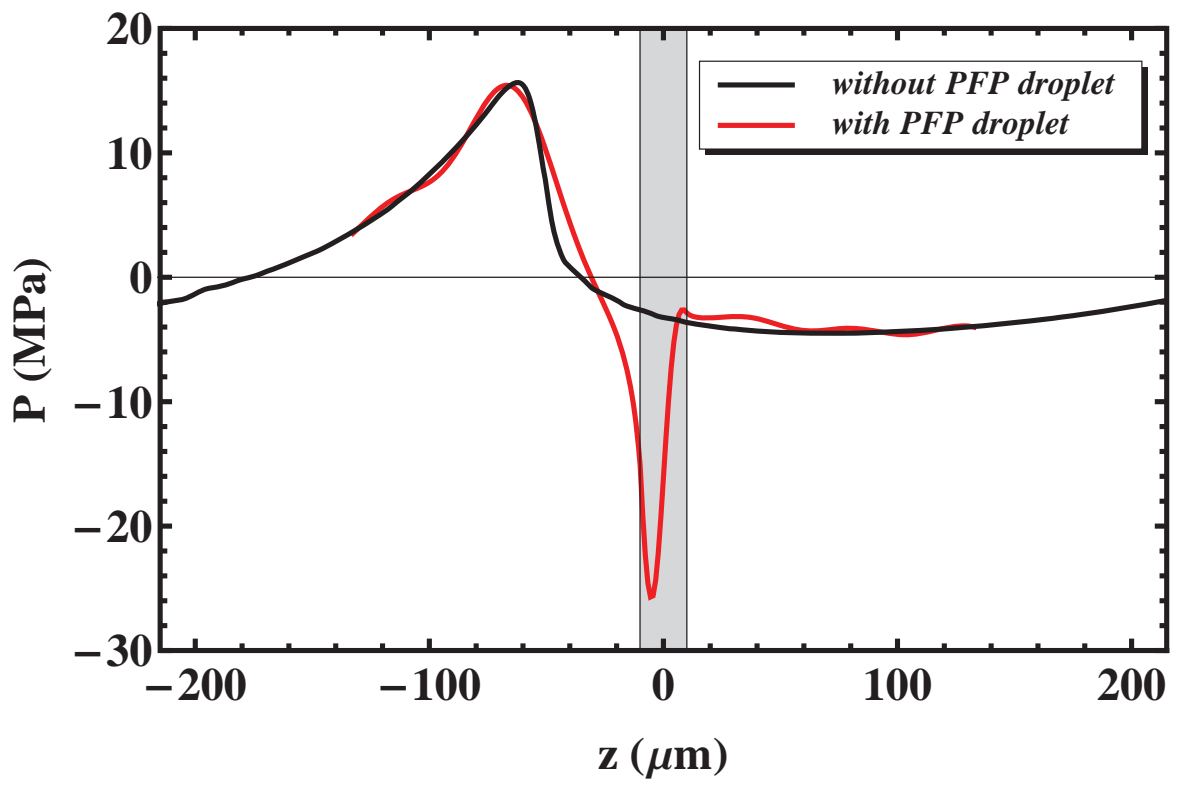

Figure 4.2: Snapshot of the superharmonic focusing effect within a spherical droplet. The black line represents the acoustic pressure waveform on the axis of symmetry $(\theta=0)$ as a function of the $z$-coordinate in the absence of a droplet. The red solid line is the focused pressure in the presence of the droplet. The snapshot is taken right at the moment of minimum focused pressure. The horizontal axis displays one full wavelength in the medium outside the droplet. The gray shaded region depicts the position of the droplet, $\mathrm{R}=10 \mu \mathrm{m}$. The focusing spot lies around $z=-0.4 \mathrm{R}$ and the pressure is amplified 5.8 times compared to the incident acoustic pressure $\mathrm{P}_{\text {inc }}^{-}=-4.5 \mathrm{MPa}$.

symmetry, where the wave propagates from left to right. The black line represents one wavelength of the wave, $\lambda_{0}=438 \mu \mathrm{m}$, in the absence of a droplet. The red line represents the same wave in the presence of a $R=10 \mu \mathrm{m}$ droplet, calculated using the real part of Eq. (4.11). The position of the droplet is indicated by the gray shaded region. In contrast to the non-diffracted (distorted) wave, the diffracted wave changes its shape depending on how far it has propagated relative to the droplet position. The red curve shows the pressure right at that particular time instant when the maximal negative pressure is reached over the full period $2 \pi / \omega$. It can be observed that a focused pressure of $\mathrm{P}_{\mathrm{drop}}^{-}=-26 \mathrm{MPa}$ is achieved within the droplet for an incoming wave with a peak negative pressure $\mathrm{P}_{\text {inc }}^{-}=-4.5 \mathrm{MPa}$. A near six-fold increase in peak 
negative pressure amplitude is observed in a concentrated region on the proximal side around $z=-0.4 \mathrm{R}$. We will call this position the focusing spot. For any given set of input parameter values the solution of Eq. (4.11) has an absolute minimum in $(r, \theta, t)$ space. Nucleation within the droplet is assumed to be most probable at such a minimum, where (and when) the focused pressure amplitude is maximal.

The pressure amplification factor in the focusing spot as well as its location depend on the input parameter values, i.e. frequency, pressure amplitude, transducer geometry and size, which prescribes the propagation distance to the focal point. Due to the nonlinear propagation of the ultrasound wave its shape, expressed in the coefficients $a_{n}$ and $\phi_{n}$, depends on the pressure amplitude. The higher the pressure, the more nonlinear the wave becomes, as the amplitudes of the superharmonics build up roughly as $\left(P_{\text {sur }}\right)^{n}$, where $P_{\text {sur }}$ is the pressure amplitude at the transducer surface and $n$ is the number of that particular harmonic. Figure 4.3a shows the dependence of the pressure amplification factor on the incident peak negative pressure $P_{\text {inc }}^{-}$at the focusing spot for droplet sizes $R=4,7$, and $10 \mu \mathrm{m}$. For instance, in the case of a $\mathrm{R}=10 \mu \mathrm{m}$ droplet, the pressure signal is not amplified until a pressure near $\mathrm{P}_{\text {inc }}^{-}=-2.5 \mathrm{MPa}$.

The focusing effect strongly depends on the frequency of the driving pressure field. Here two effects come into play. Firstly, the nonlinear propagation depends on frequency and, secondly, there is a strong coupling between the frequency and the droplet size due to diffraction. Both frequency and size appear in a combined way in the dimensionless parameters $k_{1} R$ and $k_{0} R$, see Eqs. (4.9) and (4.11). In Fig. 4.3b we plot the pressure amplification factor at the focusing spot as a function of frequency for the droplet sizes $R=4$, 7 , and $10 \mu \mathrm{m}$ driven at a peak negative pressure $P_{\text {inc }}^{-}=-4.5 \mathrm{MPa}$. It shows that the pressure is amplified with increasing frequency and this tendency is in agreement with the work of Kripfgans et al. [7].

Figure 4.4a shows the superharmonic focusing effect as a function of the droplet size. It shows resemblance to Fig. $4.3 \mathrm{~b}$ through the coupling through kR. By comparing with Fig. $4.3 \mathrm{~b}$ one can now discriminate between the effect of diffraction and nonlinear propagation. Both Figs. $4.3 \mathrm{~b}$ and $4.4 \mathrm{a}$ display the same superharmonic focusing effect, however only the first is governed by the nonlinear distortion as a function of frequency. The amount of focusing depends both on the degree of constructive interference of the various harmonics within the droplet and whether or not these harmonics are near the internal acoustic resonance of the droplet, which occurs once $k R$ is close 
to 1 . Because of this intricate interplay, the focusing effect will show rapid changes and significant peaks as a function of frequency and size, as shown in Figs. $4.3 \mathrm{~b}$ and $4.4 \mathrm{a}$.

The position of the absolute minimum pressure in Eq. (4.11) was calculated numerically by an iterative method of successive displacement along the variables $(r, \theta, t)$. The absolute minimum was found to be always on the axis of symmetry $(\theta=0)$. Figure $4.4 \mathrm{~b}$ shows how the measured position of the focusing spot depends on the droplet size for a fundamental frequency of $3.5 \mathrm{MHz}$ (red curve). For example, for droplets with radii between 1 and $5 \mu \mathrm{m}$, nucleation is expected to occur homogeneously distributed through the droplet [24], while for bubble radii between 8 and $12 \mu \mathrm{m}$ the focusing spot is located near $z=-0.4 \mathrm{R}$ and positioned at the proximal side, i.e. on the side where the nonlinear ultrasound wave hits the droplet. Note that Fig. 4.4b shows the result of the constructive interference of all superharmonics. Taking only the fundamental frequency, or any single harmonic, as in Eq. (4.10) will lead to a completely different diffraction pattern, and as a result into different focusing position and strength or to no focusing effect at all.

A total of 142 nucleation events were imaged experimentally using ultra high-speed imaging at up to 20 million frames per second [33, 34]. A typical example of such a droplet vaporization event is shown in Fig. 4.5a. Nucleation is initiated between frames 3 and 4 at the position of minimum pressure, where the focused pressure increases the chance of nucleation. Once the nucleus is formed, rapid bubble growth follows with a typical expansion velocity on the order of $\dot{R}=10 \mathrm{~m} / \mathrm{s}$ depending on the ambient temperature [22]. The position of each focusing spot was measured from the geometrical center of the growing nucleus with a $\sqrt{2}$ correction to account for the $45^{\circ}$ inclination of the optical imaging plane with the direction of US propagation (see Methods). Spatial nucleation maps were then constructed as displayed in Fig. 4.5b.

The position of the focusing spot as a function of the droplet size is summarized in Fig. 4.4b. They are in good agreement with the theoretical prediction, being more scattered for smaller droplets and highly localized for the larger ones. This is to be expected as the pressure amplification factor (from Fig. 4.4a) is only slightly above unity for the smaller droplets, leading to a stochastic random nucleation behaviour, while for the larger droplets a much higher amplification factor is achieved leading to sharp focusing of the acoustic wave and instant nucleation in a well-defined spot. Figure 4.4b was calculated for those positions with absolute minimum of pressure in the $(r, \theta, t)$ parameter space. However, one can calculate that for the larger bubbles 

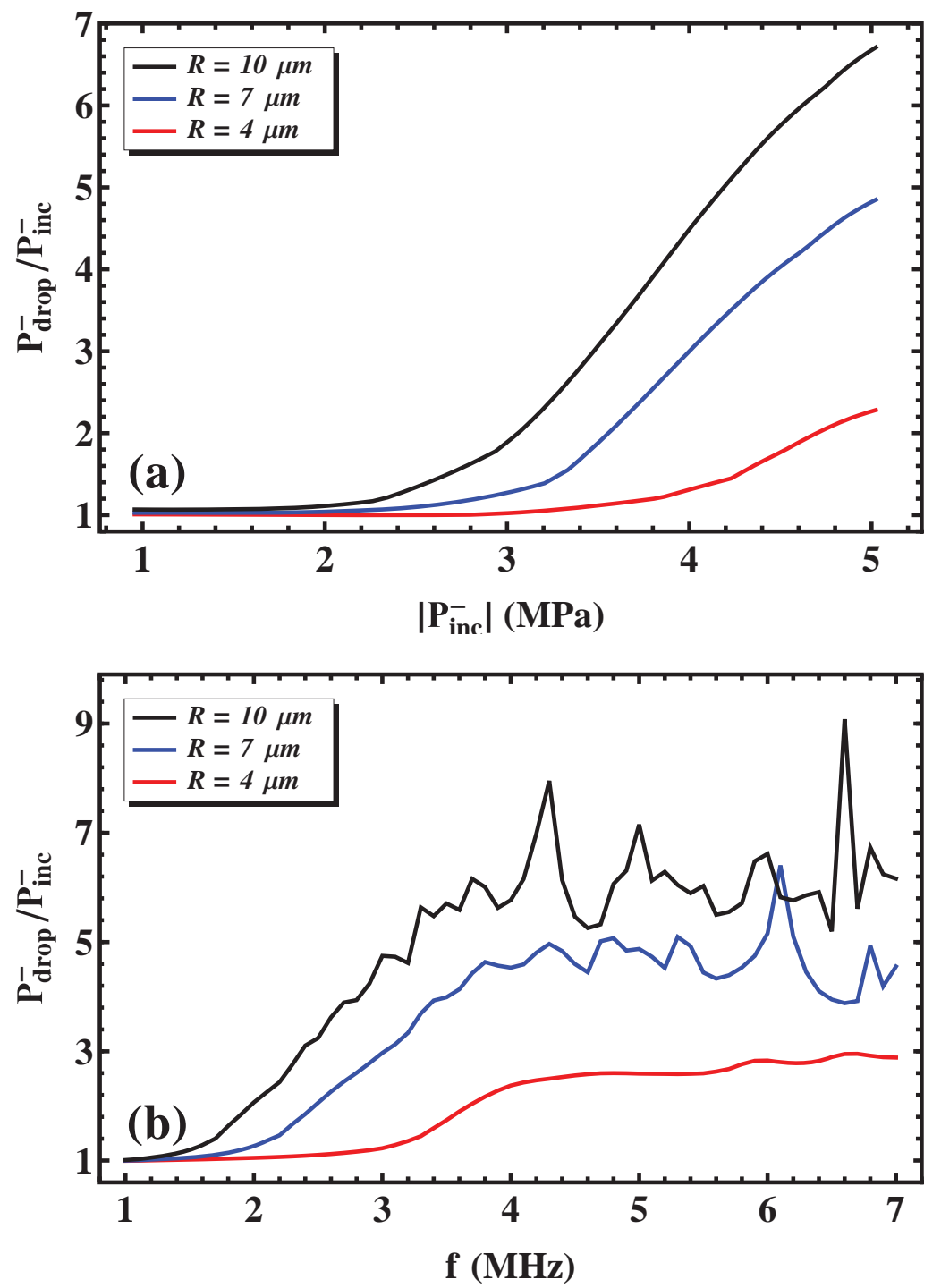

Figure 4.3: Dependence of the pressure amplification factor at the focusing spot for three microdroplet radii (a) as a function of the incident acoustic peak negative pressure $\mathrm{P}_{\text {inc }}^{-}$at a driving frequency of $3.5 \mathrm{MHz}$ and (b) as a function of the driving frequency f for a peak negative pressure of $-4.5 \mathrm{MPa}$. 
in the parameter set considered here, Eq. (4.11) has a second local minimum on the axis of symmetry around $z=+0.4$ R, i.e. on the distal side, occurring at a different time than the first one. Due to the stochastic nature of ADV, and provided that enough acoustic power is applied, i.e. above the vaporization threshold, acoustic vaporization can also be initiated at this second local minimum [22].

The nucleation maps of Fig. 4.5b show that for a driving frequency of 3.5 $\mathrm{MHz}$ (red), the smaller droplets are indeed nucleated in random position, with a tendency to focus for the sizes $6 \mu \mathrm{m}$ to $10 \mu \mathrm{m}$, whereas for the larger droplet sizes $(10-14 \mu \mathrm{m})$ the nucleation spots are highly localized. The data shows that for a fundamental driving frequency of $5.0 \mathrm{MHz}$ nucleation occurs in a focal spot already for smaller droplet sizes $(6-10 \mu \mathrm{m})$. The histogram shows the positions $\mathrm{z} / \mathrm{R}$ for $3.5 \mathrm{MHz}$ for the $10-14 \mu \mathrm{m}$ droplet sizes and 5.0 MHz for the 6-10 $\mu \mathrm{m}$ droplet sizes. For the $3.5 \mathrm{MHz}$ frequency experiment the mean position is -0.37 (standard deviation of $0.10 ; \mathrm{N}=28$ events), while the corresponding theoretical prediction is -0.45 . For the $5.0 \mathrm{MHz}$ experiment the mean position is -0.54 (standard deviation of $0.10 ; \mathrm{N}=58$ events) with a corresponding theoretical prediction of -0.59 . Thus, the focusing spot moves more proximal to the transducer for a higher frequency and its position is in quantitative agreement with the theory.

While the presented model explains the tendency of the nucleation threshold with the driving pressure, frequency of the ultrasound wave, and size of the droplets, a detailed calculation of the probability of nucleation must also incorporate parameters such as the purity of the PFP liquid, the dissolved gas concentration, liquid temperature, vapor pressure associated with it, number of US cycles, droplet concentration, droplet size distribution, etc. By using Eq. (4.11) one could match the peak negative pressure at the focusing spot (e.g. $-26 \mathrm{MPa}$ at $\mathrm{R}=10 \mu \mathrm{m}$ in Figure $4.3 \mathrm{a}$ ) with the incident peak negative pressure threshold measured in experiments as a function of these parameters. The numerical results presented here are calculated and compared to experiments performed in water. The shape of the nonlinearly distorted wave is strongly dependent on the parameters of the propagating media. For human tissue the Goldberg ratio is lower than for water [35]. This indicates that nonlinear distortion is easier to achieve in water, compared to tissue. Therefore the experiments performed in vivo are expected to have different nucleation patterns, notably with a higher nucleation threshold compared to the in vitro experiments.

The present theory shows that the larger the droplet size, the stronger the 

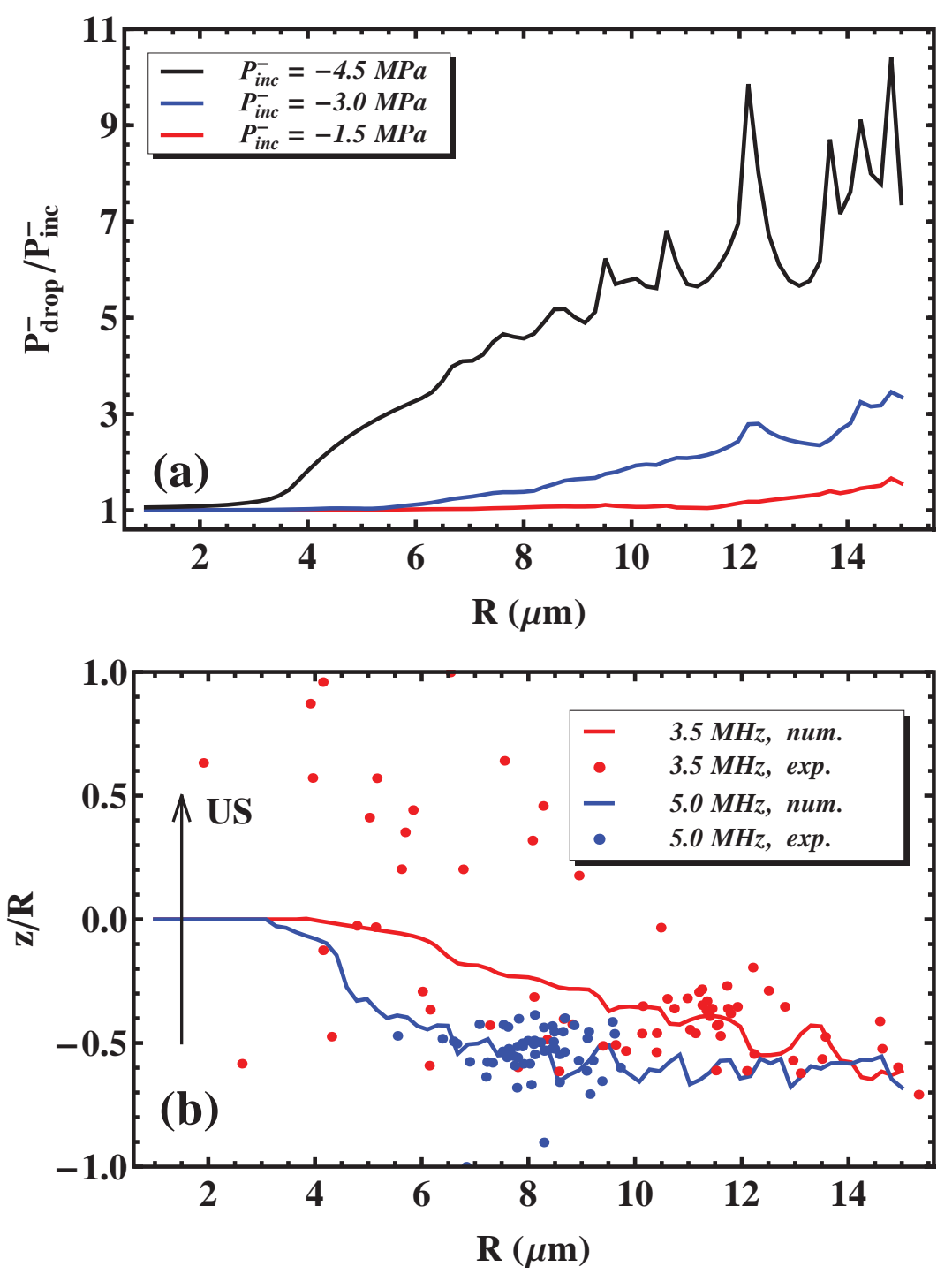

Figure 4.4: (a). The dependence of the pressure amplification factor at the focusing spot as a function of the droplet radius $\mathrm{R}$ for three incident acoustic peak negative pressures $\mathrm{P}_{\text {inc }}^{-}$(b). The position of the nucleation site as a function of the droplet size. The position is taken along the axis of US propagation on the axis of symmetry of the system. The solid lines represent the calculated positions of maximal peak negative pressure for a frequency of $3.5 \mathrm{MHz}$ (red) and $5.0 \mathrm{MHz}$ (blue), respectively. The dots represent measured nucleation spots for a range of bubble sizes for the two frequencies. 

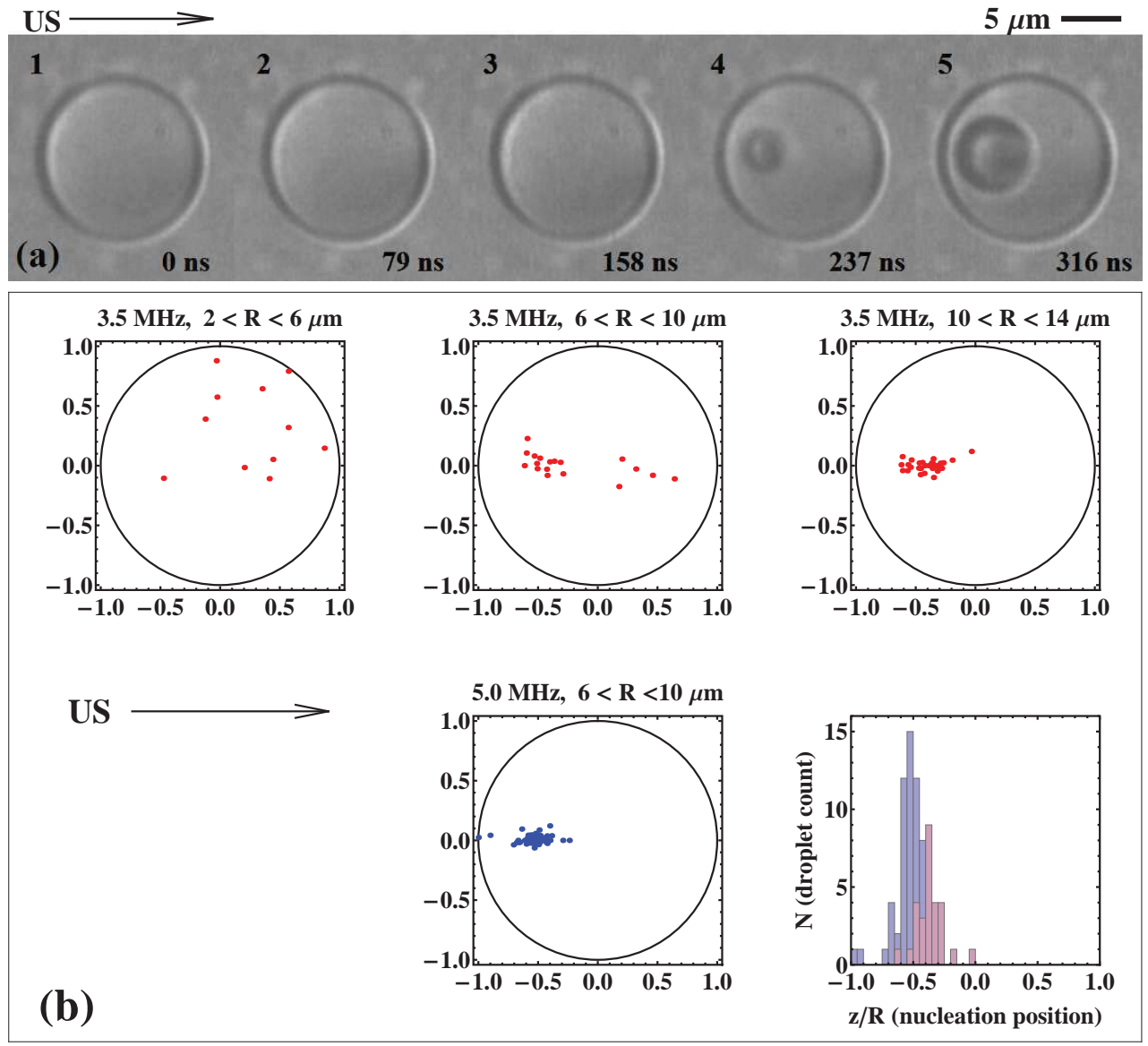

Figure 4.5: (a). A set of consecutive images showing acoustic droplet vaporization of a $7.4 \mu \mathrm{m}$ radius PFP droplet taken at a frame rate of $12.6 \mathrm{Mfps}$. The droplet is triggered by an 8-cycle $5 \mathrm{MHz}$ frequency US pulse. The nucleation is initiated between frames 3 and 4. Frames 4 and 5 show the subsequent vapor bubble growth [24]. (b). Nucleation maps for the two frequencies and for a range of droplet sizes. The histogram shows the focused positions $\mathrm{z} / \mathrm{R}$ for a frequency of $3.5 \mathrm{MHz}$ for the 10-14 $\mathrm{\mu m}$ droplet sizes and for a frequency of 5.0 MHz for the 6-10 $\mu \mathrm{m}$ droplet sizes. 
focusing. The smaller nanodroplets will require a higher frequency for activation. For the higher frequencies the harmonic content will be higher through nonlinear propagation and a higher number of superharmonics will be formed. Attenuation, however, will be stronger for higher frequencies. Thus, it is reasonable to assume that the combined effect will still be there, but probably less pronounced. A precise answer, however, cannot be provided given the computational effort of calculating nonlinear propagation effects for these high frequencies and given the lack of experimental tools for validation of pressures and amplitudes for these high frequencies and for optical imaging which would then be required at nanometer precision.

The new physical insight allows for the optimization of acoustic droplet vaporisation for therapeutic applications, in particular with respect to the acoustic pressures required for activation, thereby minimizing the negative bioeffects associated with the use of high-intensity ultrasound. This includes the design of droplets by mixing different liquids of different physical properties which allows to vary the acoustic impedance by a change of the density and speed of sound. Using dual or multiple frequency transducers, the amplitudes and phases of the transmit waves can be optimized to have maximal constructive interference within the droplets to maximize the focusing strength at any given acoustic input pressure.

\subsection{Materials}

\subsubsection{Numerics}

Several numerical codes $[31,36]$ based on the KZK equation, Eq. (4.1), are available to calculate the nonlinear pressure in the absence of a droplet. We use a numerical axisymmetric implementation written in C programming language developed in-house [37]. It has been validated with calibrated hydrophone measurements and was found to be able to represent the experimental pressure waveform with a precision of $10 \%$ [37]. The result of the simulation is the pressure field at the acoustic focus as a function of the position, time and frequency for any given transducer geometry, i.e diameter and focal distance. The following parameter values were used in the simulations: exterior medium water with speed of sound $\mathrm{c}_{0}=1520 \mathrm{~m} / \mathrm{s}$ (at $37^{\circ} \mathrm{C}$ ), density $\rho_{0}=1000 \mathrm{~kg} / \mathrm{m}^{3}$, a nonlinear coefficient $\beta=3.5$ and a sound diffusivity $\delta=4.3 \times 10^{6} \mathrm{~s}^{-1}$, interior medium PFP with speed of sound $c_{1}=406 \mathrm{~m} / \mathrm{s}$ (at $37^{\circ} \mathrm{C}$ ) and density $\rho_{1}=1660 \mathrm{~kg} / \mathrm{m}^{3}$. In the numerical simulations the 


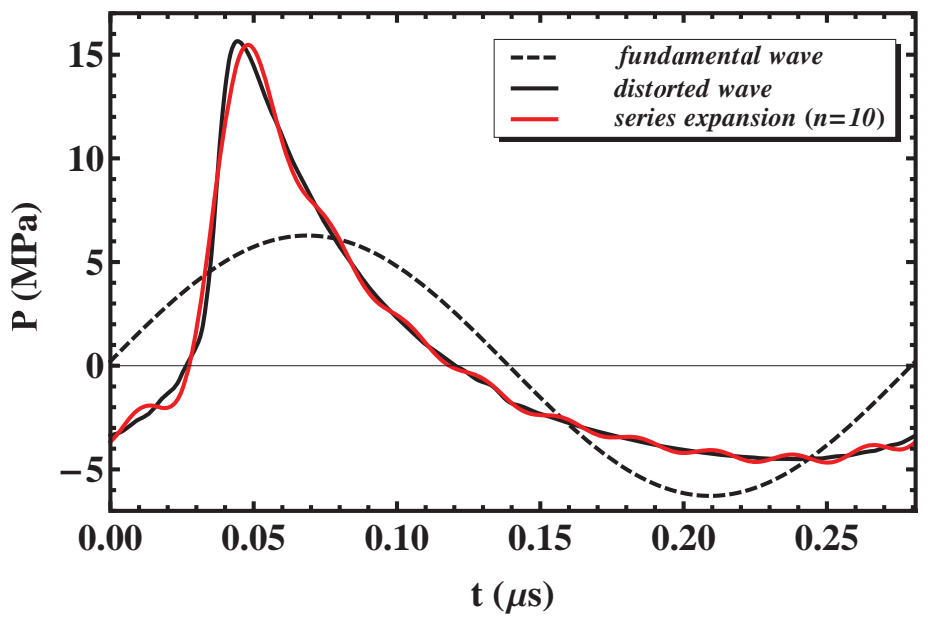

Figure 4.6: Wave distortion through nonlinear propagation. The black solid line is the pressure waveform at the focus of the transducer simulated with our KZK code [37] using Eq. (4.1). The red solid line represents ten superharmonic terms used to represent the nonlinear ultrasound pressure wave given by Eq. (4.2). The black dashed line is the first harmonic term at the fundamental frequency of the wave.

transducer dimensions were set to that used in experiment; the diameter was $19.05 \mathrm{~mm}$ (3/4 inch) and the focal distance was $38.1 \mathrm{~mm}$ (1.5 inch). The numerical code was initially run to calculate the pressure field at the focus of the transducer for a center frequency $f=3.5 \mathrm{MHz}$ and $f=5.0 \mathrm{MHz}$ for pressures ranging from 1 to $5 \mathrm{MPa}$ peak negative pressure. The code was also run at frequencies ranging from 1 to $7 \mathrm{MHz}$ with the incoming peak negative pressure at the focus kept constant at a value $P_{\text {inc }}^{-}=-4.5 \mathrm{MPa}$. Figure 4.6 shows how the series expansion approach represents the original signal depending on how many expansion terms are taken into account. We used the first ten harmonics to represent the nonlinear ultrasound pressure wave, Eq. (4.2). Equation (4.11) allows to calculate the pressure inside the droplet at any given spatial point $(r, \theta)$ at any instant of time $t$. To calculate the pressure inside the droplet, Eq. (4.11), for the parameters considered here $(1<\mathrm{R}<15 \mu \mathrm{m}$, $1<f<7 \mathrm{MHz}$ ) the first 45 terms of the series in Eq. (4.10) were used. 


\subsubsection{Experiments}

A PFP droplet emulsion was prepared using the methods adopted from Refs.[22, $23,38]$. A highly diluted suspension of droplets was injected into an OptiCell (Thermo Fisher Scientific, Waltham, MA, USA) in order to observe single microdroplets. The OptiCell was submerged in a 1-liter temperature-controlled water tank kept at a temperature of $37^{\circ} \mathrm{C}$. Microdroplets were phase-transitioned with a focused 19-mm (3/4-inch) diameter single-element transducers (Olympus Panametrics, Antwerpen, Belgium, 3.5 MHz and 5.0 MHz center frequencies, focal length $38 \mathrm{~mm}$ (1-inch)). The acoustic driving pulse was delivered from an arbitrary waveform generator (Tabor 8026; Tabor Electronics, Tel Hanan, Israel) amplified by a RF-amplifier (ENI 350L, Electronic Navigation Industries, Inc., Rochester, NY, USA). Droplet samples were vaporized with a single ultrasound pulse, consisting of a burst between 6 and $10 \mathrm{cy}$ cles and a driving pressure with a peak negative pressure $\mathrm{P}_{\text {inc }}^{-}=-4.5 \mathrm{MPa}$ at $45^{\circ}$ angle to the horizontal OptiCell plane $[22,24]$. The pressure value was calibrated in the same setup at the position of the droplets using a $0.2 \mathrm{~mm}$ PVDF probe hydrophone (DC27/000658, Precision Acoustics Ltd., Dorchester, UK). The region of interest in the Opticell was imaged with an Olympus microscope (Olympus; Zoeterwoude, The Netherlands) equipped with a $40 \times$ water-immersed microscope objective (LUMPlanFl/IR, NA $=0.8$ ) providing a resolution of $0.269 \mu \mathrm{m}$ per pixel. The sample was illuminated from below with a $65 \mathrm{~mJ}$ Xenon flash pulse (30 $\mu$ s duration) through an optical light guide (SCHOTT AG, Mainz, Germany). The timing and positions of the focusing spots within the insonified droplets were captured with the ultra high-speed framing camera Brandaris 128 [33, 34]. The camera was set to record 128 frames in a single run at a frame rate of up to 20 million frames per second (fps), thus providing an interframe time as low as $50 \mathrm{~ns}$. This allowed sampling of each cycle of the US wave with a ratio of 5-6. The uncertainty of the determination of the nucleation site from the optical images is one pixel size, which corresponds to a physical size of approximately $250 \mathrm{~nm}$. Our method assumes that nucleation occurs in the plane at a $45^{\circ}$ angle with the imaging plane and parallel with the US propagation direction. However, nucleation may occur off this plane within the depth of field of the microscope, which may well be a source of error. A more precise determination of the position of the nucleation site will require orthogonal or 3D ultra-high speed imaging, which is not available at present. 


\section{References}

[1] R. Duncan, "The dawning era of polymer therapeutics", Nat. Rev. Drug Discov. 2, 347-360 (2003).

[2] M. Ferrari, "Cancer nanotechnology: opportunities and challenges", Nat. Rev. Drug Discov. 5, 161-171 (2005).

[3] V. P. Torchilin, "Cancer nanotechnology: opportunities and challenges", Nat. Rev. Drug Discov. 4, 145-160 (2005).

[4] M. E. Davis, Z. Chen, and D. Shin, "Nanoparticle therapeutics: an emerging treatment modality for cancer", Nat. Rev. Drug Discov. 7, 771-782 (2008).

[5] N. Rapoport, A. Kennedy, J. Shea, C. Scaife, and K.-H. Nam, "Controlled and targeted tumor chemotherapy by ultrasound-activated nanoemulsions/microbubbles.", J. Contr. Rel. 138, 268-276 (2009).

[6] P. Sheeran and P. Dayton, "Phase-change contrast agents for imaging and therapy.”, Curr. Pharm. Design 18, 2152-2165 (2012).

[7] O. D. Kripfgans, J. B. Fowlkes, D. L. Miller, O. P. Eldevik, and P. L. Carson, "Acoustic droplet vaporization for therapeutic and diagnostic applications", Ultrasound Med. Biol. 26, 1177-1189 (2000).

[8] C. Wang, S. Kang, Y. Lee, Y. Luo, Y. Huang, and C. Yeh, "Aptamerconjugated and drug-loaded acoustic droplets for ultrasound theranosis.", Biomaterials 33, 1939-1947 (2012).

[9] M. Fabiilli, K. Haworth, I. Sebastian, O. Kripfgans, P. Carson, and J. Fowlkes, "Delivery of chlorambucil using an acoustically-triggered perfluoropentane emulsion.”, Ultrasound Med. Biol. 36, 1364-1375 (2010).

[10] J. Fang, C. Hung, S. Hua, and T. Hwang, "Acoustically active pefluorocarbon nanoemulsions as drug delivery carriers for camptothecin: drug release and cytoxicity against cancer cells", Ultrasonics 49, 39-46 (2009). 
[11] O. D. Kripfgans, C. Orifici, P. Carson, K. A. Ives, O. P. Eldevik, and J. B. Fowlkes, "Acoustic droplet vaporization for temporal and spatial control of tissue occlusion: a kidney study", IEEE Trans. Ultrason. Ferroelec. Freq. Contr. 52, 1101-1110 (2005).

[12] M. Zhang, M. L. Fabiilli, K. J. Haworth, F. Padilla, S. D. Swanson, O. D. Kripfgans, P. Carson, and J. Fowlkes, "Acoustic droplet vaporization for enhancement of thermal ablation by high intensity focused ultrasound", Acad. Radiol. 18, 1123-1132 (2011).

[13] N. Y. Rapoport, Z. Gao, and A. Kennedy, "Multifunctional nanoparticles for combining ultrasonic tumor imaging and targeted chemotherapy", J. Natl. Cancer Inst. 99, 1095-1106 (2007).

[14] P. Mohan and N. Rapoport, "Doxorubicin as a molecular nanotheranostic agent: Effect of doxorubicin encapsulation in micelles or nanoemulsions on the ultrasound-mediated intracellular delivery and nuclear trafficking.”, Mol. Pharmaceut. 7, 1959-1973 (2010).

[15] O. Couture, M. Faivre, N. Pannacci, A. Babataheri, V. Servois, P. Tabeling, and M. Tanter, "Ultrasound internal tattooing", Med. Phys. 38, 1116-1123 (2011).

[16] R. E. Apfel and C. K. Holland, "Gauging the likelihood of cavitation from short-pulse, low-duty cycle diagnostic ultrasound", Phys. Med. Biol. 17, 179-185 (1991).

[17] R. Williams, C. Wright, E. Cherin, N. Reznik, M. Lee, I. Gorelikov, F. S. Foster, N. Matsuura, and P. N. Burns, "Characterization of submicron phase-change perfluorocarbon droplets for extravascular ultrasound imaging of cancer", Phys. Med. Biol. 39, 475-489 (2013).

[18] T. Giesecke and K. Hynynen, "Ultrasound-mediated cavitation thresholds of liquid perfluorocarbon droplets in vitro", Ultrasound Med. Biol. 29, 1359-1365 (2003).

[19] K. C. Schad and K. Hynynen, "In vitro characterization of perfluorocarbon droplets for focused ultrasound therapy", Phys. Med. Biol. 55, 4933-4947 (2010). 
[20] T. D. Martz, P. S. Sheeran, D. Bardin, A. P. Lee, and P. A. Dayton, "Precision manufacture of phase-change perfluorocarbon droplets using microfluidics", Ultrasound Med. Biol. 37, 1952-1957 (2011).

[21] P. S. Sheeran, S. H. Luois, L. B. Mullin, T. O. Matsunaga, and P. A. Dayton, "Design of ultrasonically-activatable nanoparticles using low boiling point perfluorocarbons", Biomaterials 33, 3262-3269 (2012).

[22] O. Shpak, T. Kokhuis, Y. Luan, D. Lohse, N. de Jong, B. Fowlkes, M. Fabiilli, and M. Versluis, "Ultrafast dynamics of the acoustic vaporization of phase-change microdroplets", J. Acoust. Soc. Am. 134, 1610-1621 (2013).

[23] N. Reznik, O. Shpak, E. C. Gelderblom, R. Williams, N. de Jong, M. Versluis, and P. N. Burns, "The efficiency and stability of bubble formation by acoustic vaporization of submicron perfluorocarbon droplets", Ultrasonics 53, 1368-1376 (2013).

[24] O. Shpak, L. Stricker, M. Versluis, and D. Lohse, "The role of gas in ultrasonically driven vapor bubble growth", Phys. Med. Biol. 58, 25232535 (2013).

[25] D. T. Blackstock, "On plane, spherical and cylindrical sound waves of finite amplitude in lossless fluids", J. Acoust. Soc. Am. 36, 217-219 (1964).

[26] D. R. Bacon, "Finite amplitude distortion of the pulsed fields used in diagnostic ultrasound", Ultrasound Med. Biol. 10, 189-195 (1984).

[27] E. A. Zabolotskaya and R. V. Khokhlov, "Quasi-plane waves in the nonlinear acoustics of confined beams", Sov. Phys. Acoust. 15, 35-40 (1969).

[28] V. P. Kuznetsov, "Equation of nonlinear acoustics", Sov. Phys. Acoust. 16, 467-470 (1970).

[29] J. N. Tjotta, S. Tjotta, and E. H. Vefring, "Effects of focusing on the nonlinear interaction between two collinear finite amplitude sound beams", J. Acoust. Soc. Am. 89, 1017-1027 (1991).

[30] D. Lee and A. Pierce, "Parabolic equation development in recent decade", J. Comput. Acoust. 3, 95-173 (1995). 
[31] T. S. Hart and M. F. Hamilton, "Nonlinear effects in focused sound beams", J. Acoust. Soc. Am. 84, 1488-1496 (1988).

[32] A. Prosperetti, Advanced Mathematics for Applications (Cambridge University Press) (2011).

[33] C. T. Chin, C. Lancée, J. Borsboom, F. Mastik, M. E. Frijlink, N. de Jong, M. Versluis, and D. Lohse, "Brandaris 128: A digital 25 million frames per second camera with 128 highly sensitive frames", Rev. Sci. Instr. 74, 5026-5034 (2003).

[34] E. C. Gelderblom, H. J. Vos, F. Mastik, T. Faez, T. Kokhuis, A. F. W. van der Steen, N. de Jong, D. Lohse, and M. Versluis, "Brandaris 128 ultra-high-speed imaging facility: 10 years of operation, updates and enhanced features", Rev. Sci. Instr. 83, 103706 (2012).

[35] T. L. Szabo, F. Clougherty, and C. Grossman, "Effects on nonlinearity on the estimation of in situ values of acoustic output parameters", Ultrasound Med. Biol. 18, 33-42 (1999).

[36] Y.-S. Lee and M. F. Hamilton, "Time-domain modeling of pulsed finiteamplitude sound beams”, J. Acoust. Soc. Am. 97, 906-917 (1995).

[37] H. J. Vos, D. E. Goertz, and N. de Jong, "Self-demodulation of highfrequency ultrasound”, J. Acoust. Soc. Am. 127, 1208-1217 (2010).

[38] M. Fabiilli, K. Haworth, N. Fakhri, O. Kripfgans, P. Carson, and J. Fowlkes, "The role of inertial cavitation in acoustic droplet vaporization.”, IEEE Trans. Ultrason. Ferroelec. Freq. Contr. 56, 1006-1017 (2009). 


\section{5 Vaporization dynamics $\$$}

Acoustically sensitive emulsions are a promising tool for medical applications such as localized drug delivery. The physical mechanisms underlying the ultrasound-triggered nucleation and subsequent vaporization of these phase-change emulsions are largely unexplored. Here, the acoustic vaporization of individual micron-sized perfluoropentane (PFP) droplets is studied at a nanoseconds timescale. Highly diluted emulsions of PFP-in-water and oilin-PFP-in-water droplets, ranging from 3.5-11 $\mu \mathrm{m}$ in radius, were prepared and the nucleation and growth of the vapor bubbles was imaged at frame rates of up to 20 Mfps. The droplet vaporization dynamics was observed to have three distinct regimes: 1. prior to nucleation, a regime of droplet deformation and oscillatory translations within the surrounding fluid along the propagation direction of the applied ultrasound; 2. a regime characterized by the rapid growth of a vapor bubble enhanced by ultrasound-driven rectified heat transfer; and 3. a final phase characterized by a relatively slow expansion, after ultrasound stops, that is fully dominated by heat transfer. A method to measure the moment of inception of the nucleation event with respect to the

\footnotetext{
${ }^{\ddagger}$ Published as: Oleksandr Shpak, Tom Kokhuis, Ying Luan, Detlef Lohse, Nico de Jong, Brian Fowlkes, Mario Fabiilli, and Michel Versluis, "Ultrafast dynamics of the acoustic vaporization of phase-change microdroplets", Journal of the Acoustical Society of America 134 (2). 1610-1621 (2013).
} 
phase of the ultrasound wave is proposed. A simple physical model captures quantitatively all of the features of the subsequent vapor bubble growth.

\subsection{Introduction}

Ultrasound can be used to phase-transition liquid droplets, either submicronor micron-sized, into gas bubbles a process known as acoustic droplet vaporization (ADV) [1]. These acoustically-sensitive phase-change emulsions are composed of a volatile perfluorocarbon (PFC), such as perfluoropentane (PFP, $29^{\circ} \mathrm{C}$ boiling point), and are stabilized by a surfactant shell. Due to the boiling point elevation of the PFC when formulated as droplets [2], a PFP emulsion does not spontaneously vaporize when injected in vivo at $37^{\circ} \mathrm{C}[3,4]$. However, upon exposure to ultrasound above a certain acoustic pressure amplitude, the PFC within the emulsion is vaporized [5].

As highlighted by a recent review [6], PFC emulsions have been used in a wide variety of diagnostic and therapeutic applications. Since ultrasound can be applied non-invasively and focally, ADV has been investigated as a means of localizing drug delivery, especially for therapeutic agents with a narrow therapeutic index, such as chemotherapy drugs [7-9]. Drug release from these acoustically sensitive PFC emulsions is ideally linked to ADV, thus reducing the systemic side effects associated with conventional drug administration routes. Since PFCs are very hydrophobic and lipophobic [10], therapeutic agents are usually not dissolved directly in the PFC phase but rather in a secondary phase that is contained within the PFC emulsion [11]. Recent studies have also demonstrated that nanoscale PFC droplets may extravasate through leaky tumor vasculature, thus passively accumulating in the interstitial space and enhancing the therapeutic outcome $[12,13]$.

The complex dynamics of ADV has been previously studied using high speed microscopy for micron-sized [14-16] and submicron-sized [17] singlephase, PFC emulsions. Wong et al. [15] already denoted that ADV bubble dynamics can be described by multiple stages following empirical laws. The following three stages were proposed: 1 . a microsecond-long nucleation stage with a linear growth rate; 2 . a stage, lasting several microseconds, where a linear growth rate is observed, but smaller than the previous stage, and 3. a stage up to $200 \mu \mathrm{s}$ in duration with a nonlinear growth rate where the vapor bubble reaches $90-95 \%$ of its final size, before asymptotically reaching its final size. Owing partly to the extremely fast time scales of the phase con- 
version process, Wong et al. [15] could not resolve the complex interaction of ultrasound and bubble growth during the first two stages, which could help elucidate the physical picture. Some work on the modeling of acoustic droplet vaporization was performed by Qamar et al. [18]. They assumed that the perfluorocarbon mass evaporation rate was constant with the maximum possible value predicted by the kinetic theory limit to the mass flux that can be attained in the phase change process. Additionally, Qamar et al.used the ideal gas law for the vapor bubble. The numerical code was validated with the experimental results of Wong et al. on a time scale from 0 to $600 \mu \mathrm{s}$, and it predicted that the expansion of the bubble radius is almost linear for the first $100 \mu \mathrm{s}$.

In this paper, we use ultra-high speed optical imaging to study, for the first time, droplet vaporization dynamics at nanoseconds timescales, as well as the vaporization of a PFC double emulsion. A full frame imaging was performed here, as opposed to Kripfgans et al. [14], where the authors used a streak camera with a resolution of 17.4 nanoseconds. Moreover not only are the data sets taken at up to $20 \mathrm{Mfps}$, they also consist of 128 frames, as opposed to most other ultra high-speed imaging systems that can only do 8 , or at most 16 frames. We also develop a simple theoretical model to capture all of the growth dynamics prior to and following the nucleation event at the time scales under study here. We show that on these time scales the evaporation process is limited, not by the kinetic theory of the mass flux, but by the heat transfer from the containing liquid into the vapor bubble required to supply the latent heat for a phase conversion. We validate the model by recordings at different ambient temperatures, where we find a drastic change in growth rates due to the different temperature gradients. We observe a square root dependence of the growth rate, not linear, which is characteristic for a heat transfer limited vapor bubble expansion. We also take into account the interaction of the vapor bubble with the applied ultrasound and explain that the phase conversion rate is much more rapid due to rectified heat transfer. This result is consistent with the phenomena previously reported by Haworth et al. [19], who observed that with a two cycle pulse on a microseconds time scale only a small portion of droplet phase conversion occurred, whereas with a thirteen-cycle pulse a much larger part of the droplet was phase-converted. And finally, we recover the driving pressure information from the optical recordings and determine the exact phase of ultrasound when nucleation is initiated. 


\subsection{Materials and methods}

\subsubsection{Droplets}

Single perfluoropentane (PFP) emulsions (i.e. PFP-in-water) were prepared with bovine serum albumin using a previously published method [20] . Double PFP emulsions (i.e. oil-in-PFP-in-water) were prepared by modifying an earlier protocol [21]. The primary emulsion (oil-in-PFP) was prepared by dissolving Krytox 157 FSH (DuPont, Wilmington, DE, USA) in PFP (Strem Chemicals, Inc., Newburyport, MA, USA) at a concentration of $5.0 \%(\mathrm{w} / \mathrm{w})$. The PFP phase was then combined in a 2:1 volumetric ratio with soybean oil containing $10 \mathrm{mg} / \mathrm{mL}$ Sudan Black (Sigma Aldrich, St. Louis, MO, USA). The phases were emulsified, while in an ice bath, using the microtip accessory of a sonicator (model 450, $20 \mathrm{kHz}$, Branson, Danbury, CT, USA) operating at $125 \mathrm{~W} / \mathrm{cm}^{2}$ for 30 seconds in continuous mode. The resulting primary emulsion was added drop-wise at a 1:2 volumetric ratio to a $10 \mathrm{mg} / \mathrm{mL}$ solution of Poloxamer 188 (Sigma-Aldrich, St. Louis, MO, USA), dissolved in normal saline, which was in an ice bath and being stirred at $1100 \mathrm{rpm}$ for 10 minutes.

\subsubsection{Setup}

To study the optical response of acoustic droplet vaporization, a $10 \mathrm{~mL}$ suspension of droplets was injected into an OptiCell (Thermo Fisher Scientific, Waltham, MA, USA). The sample suspension was highly diluted (1:4000) in order to observe individual droplets. The OptiCell was submerged in a $1 \mathrm{~L}$ temperature-controlled water tank. A schematic of the experimental setup used for the droplet vaporization experiments is shown in Figure 5.1. PFP droplets were vaporized with a focused 1.5-inch diameter single-element transducer (A381S; Olympus Panametrics, Antwerpen, Belgium, 3.5 MHz center frequency, NA 1.0). The acoustic driving pulse was generated by an arbitrary waveform generator (AWG) (Tabor 8026; Tabor Electronics, Tel Hanan, Israel) amplified by an RF-amplifier (ENI 350L, Electronic Navigation Industries, Inc., Rochester, NY, USA). Droplet samples were vaporized with a single ultrasound pulse, consisting of a burst of 4 to 10 cycles in length and at a peak negative pressure (PNP) of 4.5 MPa. The Opticell was placed under a microscope equipped with a $40 \times$ water-immersion objective (LUMFPL; Olympus, Zoeterwoude, The Netherlands), coupled to the Brandaris 128 ultra-high speed imaging facility $[22,23]$ providing a spatial 


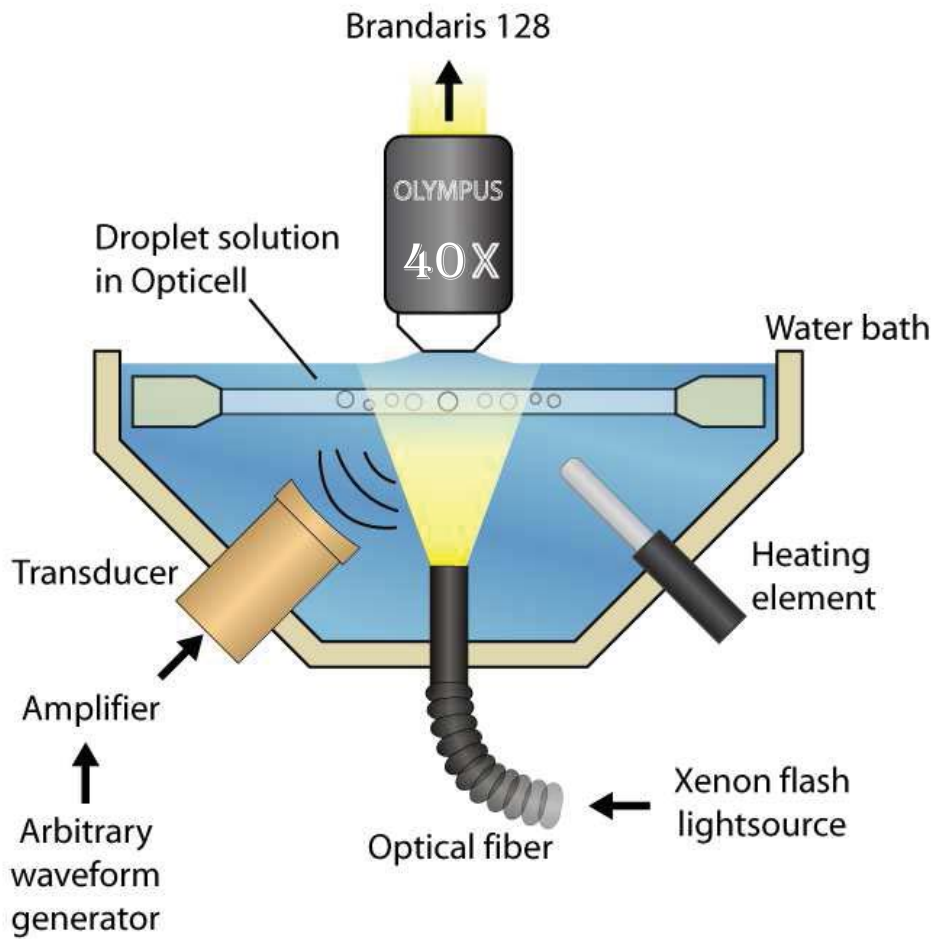

Figure 5.1: (color online) Schematics of the experimental setup. An OptiCell polystyrene container with the microdroplet suspension was kept in the water tank at $35^{\circ} \mathrm{C}-50^{\circ} \mathrm{C}$ ambient temperature. A focused transducer was sending the USpulses from a side inclined at 45 degrees to the plane of the Opticell. The samples were imaged from the top with an upright microscope coupled to the Brandaris 128 high-speed camera and irradiated from below with a xenon flash light.

resolution of $0.269 \mu \mathrm{m}$ per pixel. The camera recorded sets of 128 images at a frame rate of up to 20 million frames per second (fps) with an exposure time down to 50 nanoseconds. The camera recording started a few microseconds before ultrasound arrival.

The transducer was focused on the Opticell containing the droplet sample in the following way: the OptiCell was removed, the tip of a hydrophone (DC27/000658, Precision Acoustics LTD, Dorchester, United Kingdom) was positioned in the optical focus of the microscope objective with a XYZ - micropositioning stage, while the acoustic focus of the ultrasound transducer was aligned with a planar stage. To keep the optical and acoustic focus per- 
fectly aligned, the OptiCell chamber was connected to a 3D translation stage to allow for the positioning of the sample independently from the transducer and the microscope. A $65 \mathrm{~mJ}$ xenon flash pulse (30 $\mu$ s duration) was used for bright field illumination. The acoustic field is free from reflections from the optical components at the time of activation, as it is directed from a transducer placed at the bottom under a $45^{\circ}$ angle to the optical axis, see Fig. 5.1. Minor reflections from the microscope objective of a magnitude of approximately $5 \%$ of the incoming pressure field can be observed at $15 \mu$ s after activation, but these are outside the measurement window of the experiment.

\subsubsection{Data analysis}

The acquired optical images were analyzed using custom image processing software [24]. Contours of the droplets and bubbles were detected using an algorithm based on a minimum cost function. The bubble radius was calculated as the mean distance from the bubble center of mass to the edge of the bubble in a radial coordinate system averaged over the full $2 \pi$ angle. A total of 37 droplet vaporizations were recorded: 15 vaporizations of oil-in-PFP-inwater droplets, and 22 vaporizations for PFP-in-water droplets. Only those droplets that remained in the focus of the microscope objective throughout the image sequence were included in our analysis. Six available data sets out of the 22 PFP-in-water droplets nucleation events had well-resolved vaporization dynamics and high enough optical contrast to allow for the determination of the exact moment of nucleation inception.

The raw data of the droplet positions (x-coordinates) as a function of time (see Fig. 5.3A) are obtained directly from the Brandaris 128 image frames. They contain both a systematic and a random error. The systematic error arises from the finite alignment precision of the realignment software of the individual $128 \mathrm{CCD}$ sensors in the Brandaris 128 camera [22]. To account for the systematic error, we subtract for each frame number the first recording taken at zero pressure, resulting in Fig. 5.3B. The random error, or random walk, is due to Brownian motion. The higher the ambient temperature, the more intense the random component becomes. Since Brownian motion has a random net direction, it leads to droplets wandering off in different directions, see again Fig. 5.3B. Here we propose to use $x$-coordinate differences $\hat{x}_{i}=x_{i}-x_{i-1}$ to eliminate the random walk component. The success of this method is demonstrated in Fig. 5.3C. It shows the dependence of the oscillatory translation amplitude on the applied pressure, see for more details sec- 

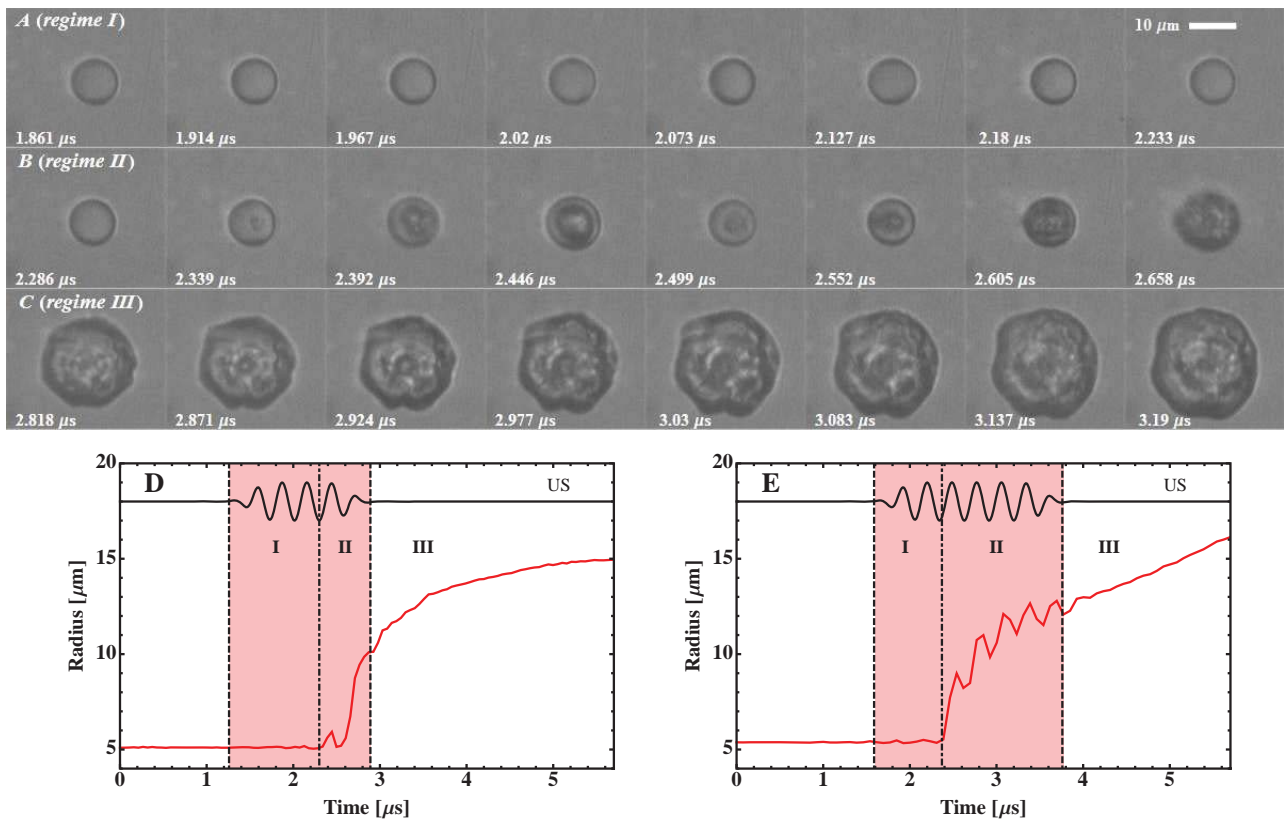

Figure 5.2: (color online) A-C. A set of consecutive images showing acoustic droplet vaporization taken at $18.5 \mathrm{Mfps}$ at an ambient temperature of $41^{\circ} \mathrm{C}$. The recordings can be categorized in three distinct regimes: I- oscillatory translations of a droplet prior to nucleation, II - vapor bubble expansion during which ultrasound is ON, and III - continued growth of the vapor bubble with ultrasound turned OFF. D-E. Two typical examples of the radius-time curves of the outer radius of the droplet/bubble complex obtained from the vaporization dynamics at $41^{\circ} \mathrm{C}$ and $50^{\circ} \mathrm{C}$ ambient temperature, respectively. The regimes I, II and III are as per the above. The radius-time curve of $D$ corresponds to the recordings shown in $A, B$ and $C$. The ultrasound pulse (US) is ON for the duration highlighted between the dashed lines. The dot-dashed line indicates the inception of nucleation. 
tion 5.3.1. The data presented here was taken at a frame rate of $13.8 \mathrm{Mfps}$ and at an ambient temperature of $44^{\circ} \mathrm{C}$.

\subsection{Results}

A typical recording of a droplet vaporization event is shown in Figure 5.2A$\mathrm{C}$. Three distinct regimes of droplet vaporization were observed in our experiments - denoted I, II, and III. Figures 5.2D and 5.2E show two examples of the measured radial dynamics of the droplet/vapor bubble system, together with the applied ultrasound pulse.

Regime I (Figure 5.2A) begins with the start of ultrasound and ends with the inception of nucleation. It is characterized by shape deformations and oscillatory translations of the droplet with a frequency of $3.5 \mathrm{MHz}$ along the propagation direction of the applied ultrasound. Regime II (Fig. 5.2B) is characterized by the growth of a vapor bubble during which ultrasound is ON. The growth of the vapor bubble in this regime is composed of two components at different time scales. The first component is a monotonic expansion with a typical velocity around $5-10 \mathrm{~m} / \mathrm{s}$ at a typical size near $10 \mu \mathrm{m}$. The second component is a sinusoidal perturbation due to successive vaporization and condensation cycles driven by the applied ultrasound. The amplitude of these oscillations $\Delta \mathrm{R}$ (determined from the average between different datasets) is around $1.2 \mu \mathrm{m}$ and the velocities are on the order of $25 \mathrm{~m} / \mathrm{s}$. Finally, regime III (Fig. 5.2C) is a continued growth of the vapor bubble, with ultrasound turned OFF, and is characterized to have only a monotonic expansion component.

\subsubsection{Oscillatory translations}

Prior to nucleation (regime I), droplets experience an oscillatory translation with a typical amplitude $\epsilon$ on the order of one hundred nanometers, with the same frequency and duration as the ultrasound transmit frequency (see Fig. 5.4A). Shape deformations are also clearly visible. From these images we extract the center of mass, or position, of the droplet $x_{i}$, where $i$ is an image number. During the oscillatory translations the droplets experience a random walk component. This component can be eliminated by looking at the discrete velocities (see Fig. 5.4B) where we plot $\hat{x}_{i}=x_{i}-x_{i-1}$ as a function of time for various pressure amplitudes. See the full description of the analysis in the 

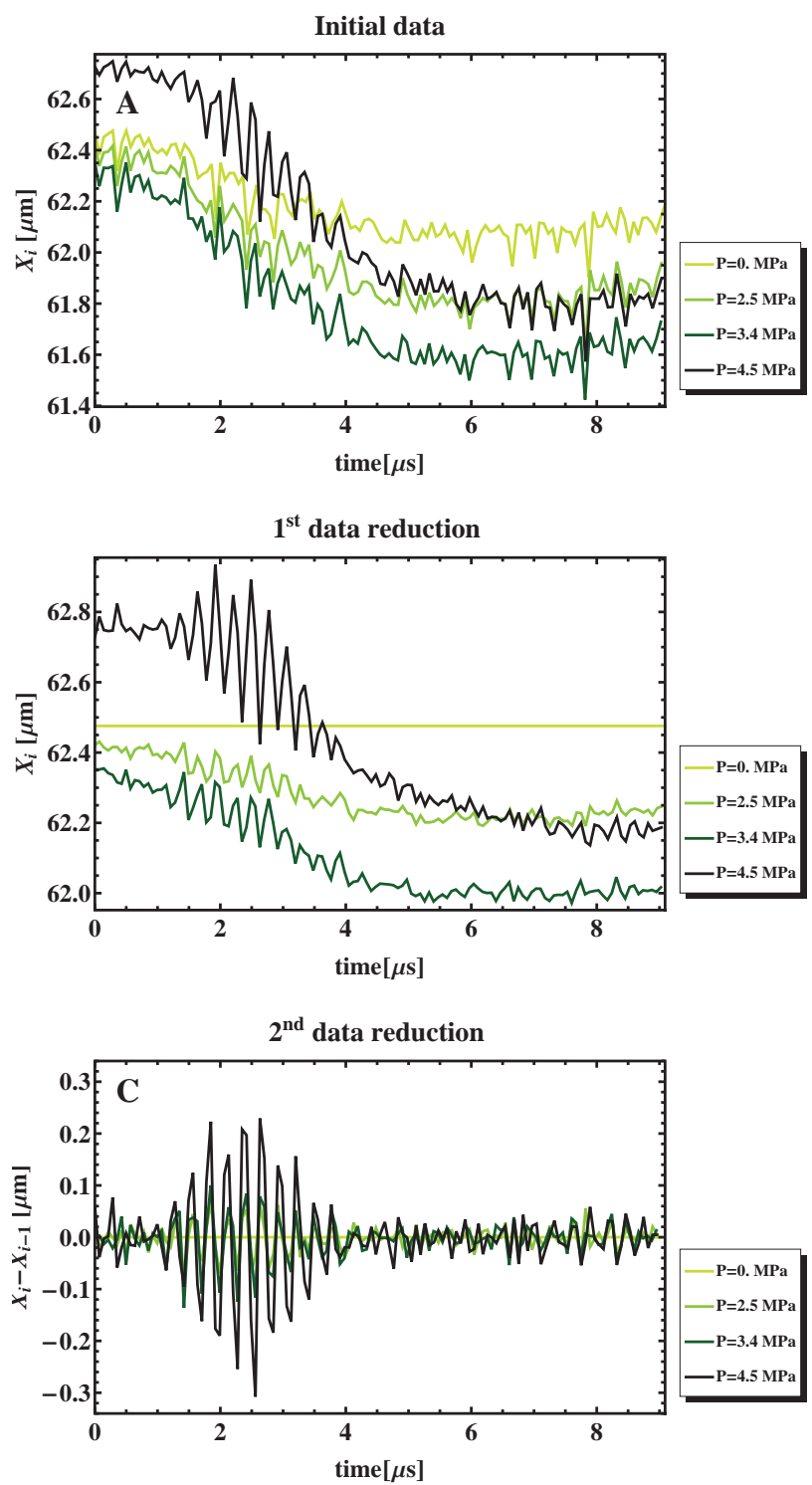

Figure 5.3: (color online) Data analysis for the oscillatory translations. A. Raw data obtained directly from the Brandaris 128 recordings. B. Systematic error removed by subtracting the reference recording at $\mathrm{P}_{\mathrm{A}}=0 \mathrm{MPa}$. C. Random error removed by taking $\mathrm{x}$-coordinate differences. 
Appendix. Then the actual amplitude of translational oscillations $\epsilon$ will be related to the introduced amplitude in discrete terms by $\hat{x}_{\max }=\epsilon(2 \pi / T) \Delta T$, where $T$ is the period of oscillations and $\Delta T=t_{i}-t_{i-1}$ is the interframe time interval. Prior to the measurement of $\hat{x}_{\max }$ a cubic spline interpolation was performed to account for a possible underestimation due to the limited sampling rate. In the case of the recording frame rate used here of $13.8 \mathrm{Mfps}$, $\hat{x}_{\max }=1.6 \epsilon$. It should be noted that the translation amplitudes $€$ are one order of magnitude lower than the ones previously observed by Kripfgans et al. [14] using streak mode imaging. This can be explained by the fact that in streak mode imaging, it is not possible to uniquely identify the direction of droplet oscillations and shape deformations may have contributed to their measured amplitude.

As seen in Figure 5.4C, higher pressures lead to higher translation amplitudes. The equation of motion of a droplet contained in another liquid, in this case water, in an acoustic field can be described by a force balance equation [25]:

$$
\frac{4}{3} \pi R^{3} \rho_{\mathrm{w}} \ddot{x}=\left(\frac{3}{2} \frac{\rho_{\mathrm{pfp}}-\rho_{\mathrm{w}}}{\rho_{\mathrm{pfp}}+\frac{1}{2} \rho_{\mathrm{w}}}-1\right) \frac{4}{3} \pi \mathrm{R}^{3} \frac{\partial \mathrm{P}}{\partial \mathrm{x}}-\mathrm{F}_{\mathrm{rad}},
$$

where the first term on the right hand side represents the added mass force and where $F_{\text {rad }}$ is the radiation force experienced by the droplet by the scattered wave due to the mismatch of acoustic impedances between the droplet and the surrounding liquid. The densities $\rho_{\mathrm{w}}=1000 \mathrm{~kg} / \mathrm{m}^{3}$ and $\rho_{\mathrm{pfp}}=1660$ $\mathrm{kg} / \mathrm{m}^{3}$ are those of water and PFP, respectively. $R$ is the droplet radius and $x$ is the spatial coordinate. For the applied pressure of $P_{A}=4.5 \mathrm{MPa}$ the amplitude of oscillatory translations $\epsilon$ measured from the recorded images is $156 \pm 30 \mathrm{~nm}$. Let us highlight that the water itself, at such high acoustic pressures, will experience periodic compressions and rarefactions with an amplitude of $\epsilon=\mathrm{P}_{\mathrm{A}} / 2 \pi \rho f c_{w}=130 \mathrm{~nm}$, where $\mathrm{f}=3.5 \mathrm{MHz}$ is the frequency and $c_{w}=1532 \mathrm{~m} / \mathrm{s}$ is the speed of sound in water. Fig. $5.4 \mathrm{C}$ depicts the liquid compressibility effect as a function of pressure and good agreement is found with the observed oscillatory motion of the droplets. Thus the compressibility of the water contributes to almost all of the oscillatory translations. No dependence on the ambient temperature was observed for the oscillatory translations in the range of $35^{\circ} \mathrm{C}$ to $50^{\circ} \mathrm{C}$ (data not shown). Following the explanation proposed here, the amplitude of oscillatory translations of other perfluorocarbon droplets is expected to be similar to the ones composed of perfluoropentane. 

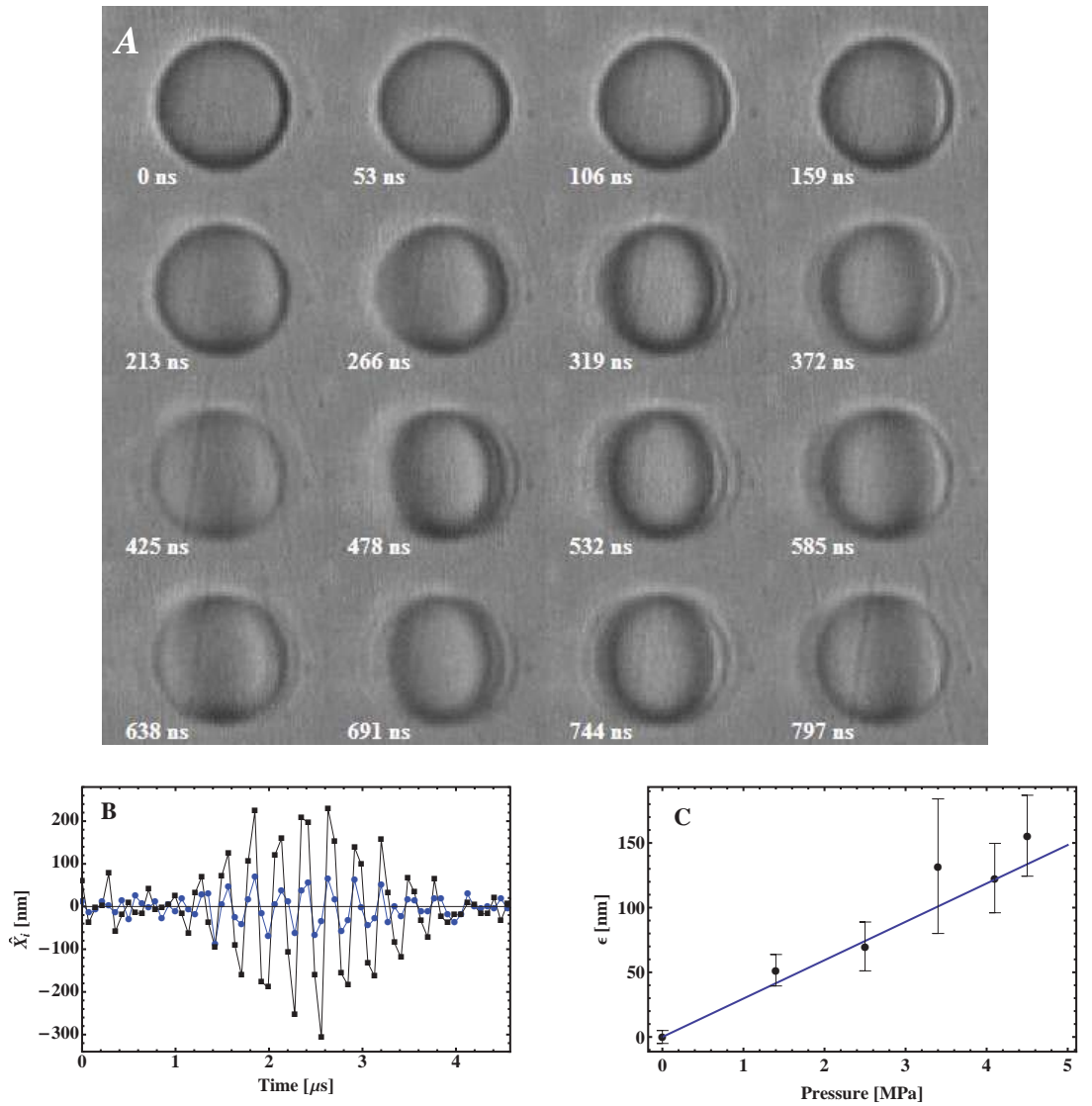

Figure 5.4: (color online) A. A set of consecutive images showing pronounced oscillations of a droplet of a radius of $11 \mu \mathrm{m}$ prior to nucleation (regime I) at an ambient temperature of $44^{\circ} \mathrm{C}$, taken at a frame rate of $18.5 \mathrm{Mfps}$. B. Oscillatory translations $\hat{x}_{i}=x_{i}-x_{i-1}$ of a microdroplet at an applied pressure amplitude of $2.5 \mathrm{MPa}$ (dots) and 4.5 MPa (squares) with the droplet not being vaporized. Ambient temperature of $44^{\circ} \mathrm{C}$. Data taken at a frame rate of $13.8 \mathrm{Mfps}$. It illustrates that the translations follow the driving ultrasound wave, which in this case constituted of a Gaussian-tapered 10 cycle pulse at a frequency of $3.5 \mathrm{MHz}$. C. Oscillatory translation amplitude $\epsilon$ as a function of the driving pressure. The line represents the theoretical oscillation amplitude of the surrounding water due to liquid compressibility. Error bars were estimated as the double standard deviation calculated over three data sets for each pressure amplitude. 

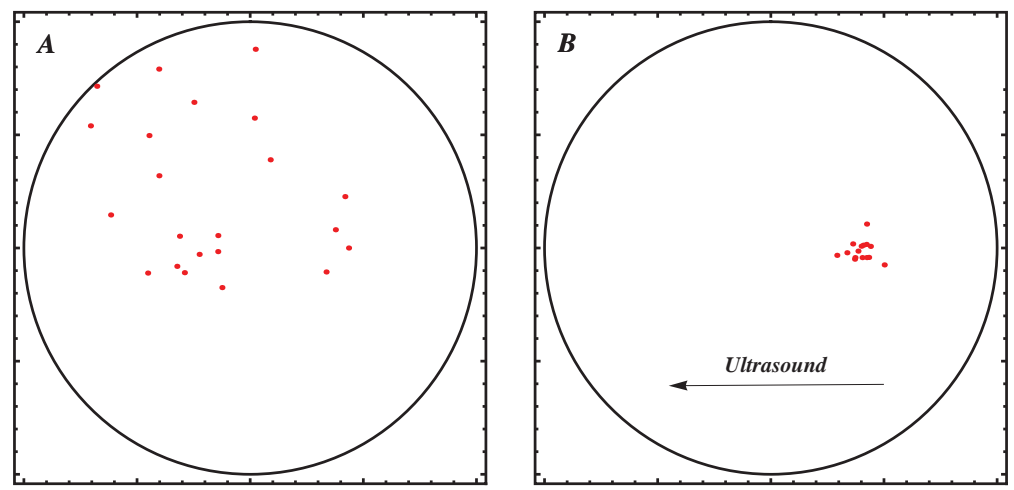

Figure 5.5: (color online) Nucleation maps constructed from 22 vaporization events for the PFC-in-water droplets $(A)$ and 16 vaporization events for oil-in-PFC-in-water droplets $(B)$. The direction of the sound wave is from right to left at $45^{\circ}$ to the plane of the image and directed towards the reader.

The aspect ratio of the maximum shape deformation of the droplet at $\mathrm{P}_{\mathrm{A}}$ $=4.5 \mathrm{MPa}$ was $16 \%$ which is in agreement with the experimental results obtained by Kripfgans et al. [14] where the authors explained the droplet shape deformation by a droplet levitation theory developed by Lee et al.[26]. Shape deformations were found to be more pronounced for larger droplets; this however will be the subject of a future study.

\subsubsection{Nucleation}

Nucleation is a highly stochastic process. It was observed that the droplets could nucleate on a second pressure cycle as easily as during a third or fourth, resulting in the fact that either regime I or II could consist of only one single ultrasound cycle (compare for example Figs. 5.2D and 5.2E). Within the time frame of one day of experiments, during which the alignment of the transducer was not changed, eight successful vaporizations were traced, one of which nucleated during the $1^{\text {st }}$ cycle, three during the $2^{\text {nd }}$, one during the $3^{\text {rd }}$ and three during the $4^{\text {th }}$ cycle. The reason that droplet vaporization was observed less frequently in the first rarefactional half-cycle may be a result of transient behavior of the transducer, leading to lower pressures in the first few cycles.

Figure 5.5 shows nucleation maps for the two compositions of droplets. For the PFC-in-water droplets nucleation occurred exclusively in the bulk of 

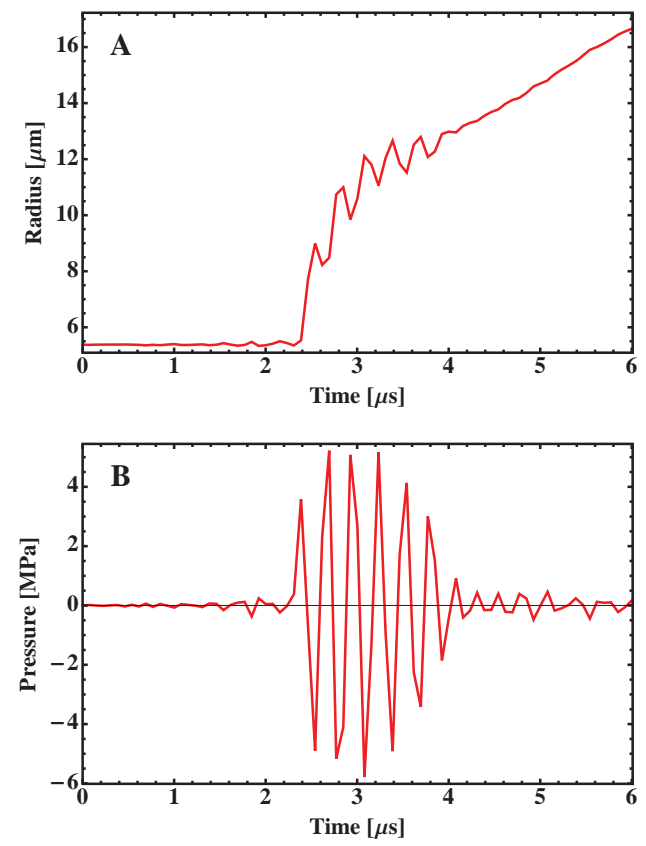

Figure 5.6: (color online) A. The radius-time curve of a droplet vaporization event at an ambient temperature of $50^{\circ} \mathrm{C} . \mathrm{B}$. The corresponding restored discrete pressure information obtained from the Rayleigh-Plesset equation. The pressure pulse consisted of 6 cycles of ultrasound with a pressure of $4.5 \mathrm{MPa}$, which is well recovered from the experimental radius-time curve.

the droplet scattered around the center and not necessarily near the interface of the two liquids. For the oil-in-PFC-in-water droplets the nucleation sites were localized close to one single point facing the incident ultrasound wave. In our experiments, the nucleation sites in the single phase droplets were not centered along the translational motion of the droplet, as was reported by Kripfgans et al. [14]. The mean size of the 22 PFP-in-water droplets from which the nucleation map was constructed was $5.6 \mu \mathrm{m}$ with a standard deviation of $2.1 \mu \mathrm{m}$, while the mean size of the 16 oil-PFP-in-water was $13.5 \mu \mathrm{m}$ with a standard deviation of $2.4 \mu \mathrm{m}$. Thus, the size difference may also be responsible for the different nucleation maps observed for the two different formulations.

While the series of obtained droplet vaporization recordings clearly show the number of cycles sent (first based on the number of oscillatory transla- 
tion cycles made and later based on the number of vaporization/condensation cycles of the vapor bubble), we lack information on the exact phase of ultrasound in each single frame due to the phase difference of ultrasound and the resulting oscillations and translations. The timing cannot simply be recovered from the trigger times of the system and knowing the speed of sound. For example, in order to account for the propagation delay time and to give an accuracy of a $25 \mathrm{~ns}$ phase shift out of a $28 \mu$ s travel time, one needs to know the speed of sound with better than 0.001 precision. With an error in the temperature reading of $1 \mathrm{~K}$, and with the potential presence of temperature gradients there is no chance of achieving such accuracy. Moreover, the needle hydrophone has a size that is two orders of magnitude larger than the typical size of the droplet, which makes the positioning of the ultrasound wave with respect to the exact position of the droplet impossible. Therefore, our goal here would be to restore the pressure information in-situ from the optical images of the vapor bubble oscillations dynamics.

Let us first assume a spherical symmetry for the entire vaporization process. The bubble dynamics is described by the Rayleigh-Plesset equation [27]:

$$
\frac{\Delta \mathrm{P}}{\rho_{w}}=\mathrm{R} \ddot{\mathrm{R}}+\frac{3}{2} \dot{\mathrm{R}}^{2},
$$

where $\Delta \mathrm{P}$ is the pressure difference between the inside and the outside of the vapor bubble while $R, \dot{R}$, and $\ddot{R}$ are the radius, radial velocity and radial acceleration of the vapor bubble, respectively. The vapor overpressure and the pressure contributions from viscous damping and surface tension are neglected here since these terms are much smaller than the driving pressure of 4.5 MPa. The radius obtained experimentally $R_{i}$ is a time series data consisting of 128 points; its number is limited by the number of optical channels in the Brandaris 128 camera. Time series data $\dot{R}_{i}$ and $\ddot{R}_{i}$ can be obtained by taking the time derivatives of $R_{i}$. Analogously, one can construct a discrete set of overpressure data $\Delta \mathrm{P}_{i}$ using Eq. 5.2. $\Delta \mathrm{P}_{i}$ consists of 126 points with a sampling rate of approximately 3-5 per cycle.

Figure 5.6 shows the result of the pressure restoration method with a $5.3 \mu \mathrm{m}$ radius droplet at an ambient temperature of $44^{\circ} \mathrm{C}$. The droplet was excited with a 6-cycle acoustic wave at a peak negative pressure amplitude of 4.5 MPa. Fig. 5.6A shows the radius of the droplet as a function of time and Fig. 5.6B its restored pressure. It can be seen that the number of cycles and the pressure amplitude are nicely recovered.

The pressure restoration method recovers only the cycles of ultrasound 


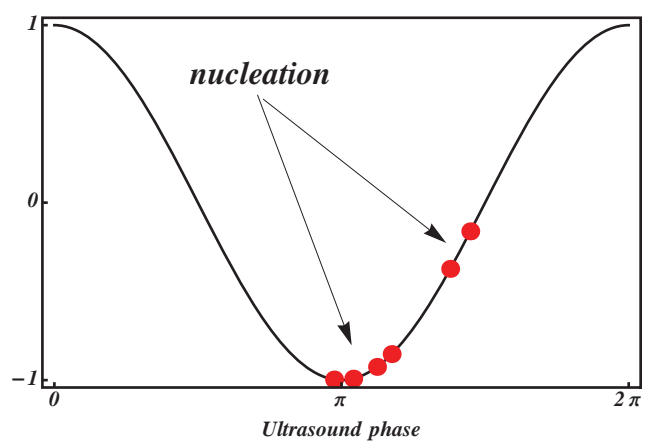

Figure 5.7: (color online) The phase of the nucleation moments based on 6 experiments using the pressure restoration information. The onset of nucleation occurs at the peak negative cycle of ultrasound.

after the onset of nucleation (in regime II). The phase of ultrasound during droplet shaking (regime I) and the phase of ultrasound during inception of the nucleation itself can be obtained by cross-correlating the input pressure pulse with the restored pulse. Interpolation of the discrete pressure pulse $\Delta \mathrm{P}_{\mathrm{i}}$ allowed us to determine the exact phase of ultrasound of each single optical image, viz. the images of nucleation. Figure 5.7 is the corresponding temporal nucleation map based on 6 processed vaporizations by the above method. Nucleation occurs during the peak negative cycle, shifted slightly towards the end of it.

\subsubsection{Vapor bubble growth}

There are three physical mechanisms that govern the vapor bubble growth process: phase transition, heat transfer and inertia. Let us first consider vapor bubble growth in regime III when ultrasound is turned OFF. The ambient temperature within the water tank was controlled and varied in the range of $35^{\circ} \mathrm{C}$ to $50^{\circ} \mathrm{C}$. The temperature of the PFP vapor bubble can be determined from the pressure vs. temperature P-T relation along the phase boundary in the phase diagram and which is given by the Clausius-Clapeyron relation. At atmospheric conditions, when the vapor pressure is $100 \mathrm{kPa}$, the vapor temperature will be at the boiling point, which is $29^{\circ} \mathrm{C}$. However, the vapor pressure may be higher and it will enable the vapor bubble to expand by pushing away the surrounding liquid (governed by the inertia equation). In turn, a 
temperature mismatch will cause heat to be transferred from the surrounding liquid to the vapor bubble (governed by the heat transfer equation) and as such supply the latent heat required for evaporative cooling (governed by the phase transition equation). Moreover, the heat transfer is complicated by the temperature distribution outside the vapor bubble, which is changing with time due to heat diffusion, and which is also affected by the geometrical expansion of the bubble, described by the continuity equation. All these processes are interconnected and are even more complicated in regime II when the system is also driven by the acoustic pressure wave.

Let us first assume that the temperature distribution inside the bubble is homogeneous and that we assume spherical symmetry of the system. By applying the method of pressure restoration, Eq. 5.2, we find that the overpressure between the inside and outside of the vapor bubble in regime III is small (of order $5 \mathrm{kPa}$ ), meaning that the inertial processes are happening fast enough to allow the vapor bubble growth and are not limiting this process. This allows us to make the hypothesis that the vapor temperature inside is constant (at a temperature of $29^{\circ} \mathrm{C}$ ) and that the vapor bubble growth is limited by heat transfer. The thermal diffusion length inside the PFP liquid

$$
\delta_{\text {diff }}=\sqrt{\frac{D}{\omega}}
$$

where $\mathrm{D}=3.21 \times 10^{-8} \mathrm{~m}^{2} / \mathrm{s}$ is the thermal diffusivity of PFP [28], and $\omega=2 \pi f$ for the frequency $f=3.5 \mathrm{MHz}$. Here, $\delta_{\text {diff }}$ is $38 \mathrm{~nm}$, which is two to three orders of magnitude smaller than the typical vapor bubble radius, with values of 5 to $30 \mu \mathrm{m}$ in the regimes II and III. This justifies the use of a thin thermal boundary layer assumption. A more precise calculation of the heat diffusion problem outside a moving sphere can be found in Plesset et al. [29].

We put the effective thermal boundary layer equal to

$$
\delta_{\text {eff }}=\sqrt{\mathrm{Dt}}
$$

then the heat transfer $W_{1}$ inside the vapor bubble from the surrounding liquid is

$$
W_{1}=4 \pi R^{2} k \frac{\Delta T}{\sqrt{D t}}
$$

where $k$ is the heat transfer coefficient, $4 \pi R^{2}$ is the interfacial area, $\Delta T$ is the temperature difference between the containing liquid and the interior of 
the bubble, and $\Delta T / \sqrt{\mathrm{Dt}}$ is the temperature gradient over the thermal boundary layer.

The latent heat energy per unit time $W_{2}$ required to supply the vapor bubble growth is:

$$
W_{2}=4 \pi R^{2} L \rho_{v} \frac{d R}{d t},
$$

with $L$ the latent heat and $\rho_{\mathrm{v}}$ the density of the PFP vapor. Equalizing Eqs. 5.5 and 5.6 and integrating with the initial condition $R\left(t=t_{0}\right)=R_{0}$ gives the radial dynamics of the vapor bubble growth:

$$
R(t)=2 \frac{k \Delta T}{L \rho_{v}} \sqrt{\frac{t-t_{0}}{D}}+R_{0}
$$

A more elaborate calculation, which takes into account the influence of increasing interfacial area on the temperature gradient, gives the relation

$$
R(t)=2 \alpha \frac{k \Delta T}{L \rho_{v}} \sqrt{\frac{t}{D}}
$$

for $\mathrm{t}_{0}=0$ and $\mathrm{R}_{0}=0$, and where $\alpha=\sqrt{3 / \pi} \approx 0.98$ calculated by Plesset and Zwick [30], or $\alpha=\sqrt{\pi / 2} \approx 0.89$ by Forster and Zuber [31]. Figure 5.8 shows that Eq. 5.7 can be fitted to the experimental data after adjusting $t_{0}$. Overall, the square-root-behavior of the radius-time curves in regimes II and III is a proof of the validity of the thermal boundary layer assumption. Regime II, however, has an even better agreement after applying the rectified heat transfer correction, see the following section 5.3.4.

\subsubsection{Ultrasound-driven vapor bubble growth}

The problem complicates when ultrasound is ON. From Fig. 5.2E for example it can be observed that following nucleation, the vapor bubble experiences a series of vaporization and recondensation cycles at the frequency of applied ultrasound. The rapid growth of the bubble in regime II as compared to regime III suggests another interesting phenomenon called rectified heat transfer and was first mentioned by Trammell[32]. Rectified heat transfer is the net effect of a decrease of the heat transfer during the ultrasound half cycle when the vapor bubble surface contracts, which is lower than the increase of the heat transfer during the half cycle when the surface expands. Several authors 


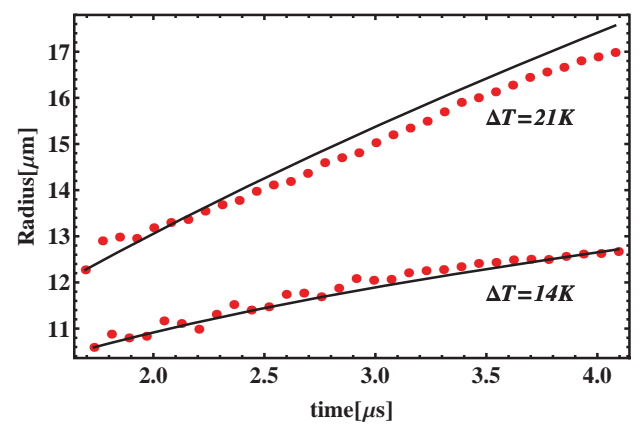

Figure 5.8: (color online) Experimental growth curves (dots) of two droplets in the heat transfer limited regime III with ultrasound OFF at two settings of superheat: $14 \mathrm{~K}$ and $21 \mathrm{~K}$. The corresponding theoretical curves (solid lines) are following Eq. 5.6. The parameters used here are $\mathrm{k}=0.056 \mathrm{~W} / \mathrm{m}^{2} \mathrm{~K}, \mathrm{D}=3.21 \times 10^{-8} \mathrm{~m}^{2} / \mathrm{s}$, and $\mathrm{L}=116.8 \mathrm{~kJ} / \mathrm{kg}$ and the good agreement was found by only fitting the value of $\mathrm{t}_{0}$.

worked on this problem: a linear solution to the equations governing the radial motion of a vapor bubble in a harmonic acoustic field was given by Finch and Neppiras [33], Wang [34] assembled a theory of rectified heat transfer, while Hao and Prosperetti [35] developed a numerical code.

Here we apply the calculation for the case of an ultrasound-driven PFP vapor bubble. Let us consider that the radius of the vapor bubble changes from $R_{0}$ to $R$. The change of the thin thermal boundary layer from $\delta_{0}$ to $\delta$ is then calculated from continuity: $4 \pi R^{2} \delta=4 \pi R_{0}^{2} \delta_{0}$.

$$
\delta=\delta_{0} \frac{R_{0}^{2}}{R^{2}}
$$

With the temperature difference $\Delta T$ as before, the temperature gradient $\Delta T / \delta$ will then be changed by a factor $R^{2} / R_{0}^{2}$, hence the overall heat transfer W changes by a factor $R^{4} / R_{0}^{4}$ :

$$
W=4 \pi R^{2} k \frac{\Delta T}{\delta}=4 \pi R^{4} k \frac{\Delta T}{\delta_{0} R_{0}^{2}}=W_{0} \frac{R^{4}}{R_{0}^{4}} .
$$

Let us now assume that the bubble with initial radius $R_{0}$ experiences sinusoidal oscillations with a small amplitude $\Delta R \ll R_{0}$ and which are fast enough that the temperature gradient and consequently $W_{0}$ does not change during a single cycle: 


$$
R=R_{0}+\Delta R \sin \omega t
$$

The net heat transfer averaged over one period of oscillations $T=2 \pi / \omega$ is then calculated from

$$
\langle W\rangle=\frac{1}{T} \int_{0}^{T} W d t=\frac{\omega}{2 \pi} \frac{W_{0}}{R_{0}^{4}} \int_{0}^{T} R^{4} d t,
$$

leading to

$$
\langle W\rangle=W_{0}\left(1+3\left(\frac{\Delta R}{R_{0}}\right)^{2}+\frac{3}{8}\left(\frac{\Delta R}{R_{0}}\right)^{4}+\cdots\right),
$$

$\langle W\rangle$ can be understood as the increased energy flow due to rectified heat transfer and with a contribution of the order of $\left(\Delta R / R_{0}\right)^{2}$.

Equation 5.13 can now be applied to correct the heat transfer $W_{1}$ inside the vapor bubble from the surrounding liquid during the time period when ultrasound is ON. Neglecting the higher order terms and keeping only the terms of order $\left(\Delta R / R_{0}\right)^{2}$, the energy balance equation $W_{1}=W_{2}$ becomes:

$$
L \rho_{v} \frac{d R}{d t}=k \frac{\Delta T}{\sqrt{D t}}\left(1+3\left(\frac{\Delta R}{R_{0}}\right)^{2}\right) .
$$

Integrated at the initial conditions $R\left(t_{0}=0\right)=0$ gives the implicit relation expressing the dependence:

$$
R-\Delta R \sqrt{3} \arctan \left(\frac{R}{\Delta R \sqrt{3}}\right)=2 \frac{k \Delta T}{L \rho_{v}} \sqrt{\frac{t}{D}} .
$$

Figure 5.9A shows the theoretical growth curves for a superheat temperature of $21 \mathrm{~K}$. The dash-dotted line represents the pure heat transfer limited process and the solid line represents the heat transfer process combined with the rectified heat transfer correction due to the fast ultrasound-driven radial oscillations. The experimental growth curve is also plotted and we find very good agreement with the rectified heat transfer model. In Fig. 5.9B we plot the growth curves (time-averaged over one cycle of ultrasound) at various degrees of the superheat $\Delta T$. For ease of interpretation we scale the graphs by the amount of superheat $\Delta \mathrm{T}$. For the heat transfer limited growth without rectified heat transfer (dot dashed line) the growth curves collapse onto each 

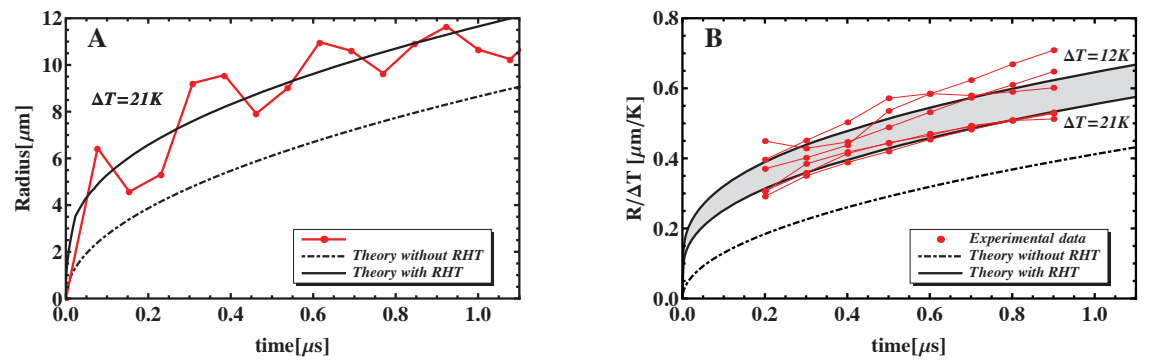

Figure 5.9: (color online) A. Radial expansion of the vapor bubble at a superheat of $21 \mathrm{~K}$ in regime II, with ultrasound $\mathrm{ON}$. Dots represent the experimental data. The dashed line is a fit to the heat transfer limited growth without applying the rectified heat transfer correction, while the solid line is with the rectified heat transfer correction applied. B. Radial growth normalised to the amount of superheat $\Delta \mathrm{T}$ between $12 \mathrm{~K}$ and $21 \mathrm{~K}$ as a function of time. Experimental data (dots) for $\Delta \mathrm{T}=12 \mathrm{~K}, 13 \mathrm{~K}$, $14 \mathrm{~K}, 15 \mathrm{~K}, 18 \mathrm{~K}$ and $21 \mathrm{~K}$ are plotted and show good agreement with the rectified heat transfer model.

other and the growth rate depends solely on $\sqrt{t}$, see Eq. 5.7. With the rectified transfer correction applied, small differences for the growth curve are found as these do not scale directly with $\Delta T$, see the implicit relation with $R$ in Eq. 5.15. Here the theoretical growth curves are plotted (grey shaded area) in the range between $\Delta T=12 \mathrm{~K}$ (highest growth curve) and $\Delta T=21 \mathrm{~K}$ (lowest growth curve). Note that the growth curve at $\Delta \mathrm{T}=21 \mathrm{~K}$ is in fact lower than at $\Delta \mathrm{T}=12 \mathrm{~K}$ as a result of the scaling with $\Delta \mathrm{T}$, whereas in absolute terms it is of course higher than at $\Delta T=12 \mathrm{~K}$. We also plot the experimental (one-cycle averaged) growth curves for $\Delta \mathrm{T}=12 \mathrm{~K}, 13 \mathrm{~K}, 14 \mathrm{~K}, 15 \mathrm{~K}, 18 \mathrm{~K}$ and $21 \mathrm{~K}$ and these show the overall very good correspondence with the rectified heat transfer correction model.

\subsection{Discussion}

This study presents, for the first time, measurements of the ultrafast vaporization dynamics of phase-change microdroplets while being able to capture prenucleation, nucleation, during US and after US, all for a single vaporization event. Overall we find very good agreement with a simple physical model describing the local heat transfer and ultrasound-driven rectified heat transfer. 
The proposed method of pressure restoration, Eq. 5.2, gives us the exact moment of ADV nucleation with respect to the phase of the ultrasound wave. The tendency of the nucleation events being shifted towards the end of the peak negative half-cycle may be explained by the fact that homogeneous nucleation is a stochastic process and that a longer time during which the liquid is stretched with a negative pressure will lead to a higher probability of nucleation. Another effect that may contribute to the scatter across the negative peak cycle is that the nucleus needs some time to grow before it can be optically detected. In its initial stage, during the first $10 \mathrm{~ns}$, a typical velocity of a vapor nucleus expansion can be calculated from the governing RayleighPlesset equation by considering that the pressure does not change much on these time scales. A numerical solution of Eq. 5.2 at a constant pressure of 4.5 $\mathrm{MPa}$ indicates that after approximately $2 \mathrm{~ns} \dot{R}$ will reach its constant terminal velocity. One can derive an analytical formula for $\dot{R}_{\text {terminal }}$ from Eq. 5.2 by substituting $\ddot{R}=0$ :

$$
\dot{\mathrm{R}}_{\text {terminal }}=\sqrt{\frac{2}{3} \frac{\Delta \mathrm{P}}{\rho_{\mathrm{pfp}}}}
$$

This estimate gives an expansion rate of the nucleus of $40 \mathrm{~m} / \mathrm{s}$ and is in agreement with what is observed in experiments (Fig. 5.2B, regime II ) where the initial nucleus growth was also about $40 \mathrm{~m} / \mathrm{s}$. In contrast, the outer radius (in what we call the droplet radius) is 5 to $10 \mathrm{~m} / \mathrm{s}$ as mentioned before depending on the droplet size. If the lower limit of detection of the vapor nucleus is taken to be 1 pixel, which is on the order of $300 \mathrm{~nm}$, then the time required to grow to this size is about $7 \mathrm{~ns}$. With the Brandaris 128 camera operating at a typical frame rate of $20 \mathrm{Mfps}$, the corresponding exposure and interframe time is $50 \mathrm{~ns}$. Thus the error the moment of nucleation relative to the phase of the ultrasound wave is due to the combined effect of the limited sampling rate of the camera and its relatively long exposure time.

During the first microsecond a monotonic expansion component was observed with typical velocities near $5-10 \mathrm{~m} / \mathrm{s}$ and with characteristic vapor bubble radii of up to $10 \mu \mathrm{m}$. These numbers agree well with experimental data reported previously for similar experiments by Wong et al. [15] (Fig. 3 in their paper) and Haworth et al.[19] (Fig. 4 in their paper). In general terms, one can calculate the velocity at any given moment of time for the time scales considered using Eq. 5.14. The numbers observed do not agree with the theoretical prediction by Qamar et al. [18]. They predict that the growth of the 
bubble is linear in time with an expansion rate near $0.3 \mathrm{~m} / \mathrm{s}$ (Fig. 2 in their paper). Our experimental data shows a square-root dependence instead with much larger expansion rates. Let us note, however, that our simplified model is applicable only for the first few microseconds post-nucelation. Later on the temperature field around the vapor bubble diffuses further away, becomes more complex and cannot be described by the thin thermal boundary layer assumption expressed in Eq. 5.3.

The spatial information of nucleation events is presented in so-called 2D nucleation maps, see Fig. 5.5. It shows that for PFP-in-water microdroplets the nucleation points are randomly scattered (albeit with most of them concentrated in the upper left segment away from ultrasound), with few events near the interface of the two liquids, suggesting a physical description based on homogeneous nucleation theory. The system with oil-in-PFP-in-water clearly has a very different distribution of nucleation event, concentrated around a single point at coordinates $\left(0.4 \mathrm{R}_{0}, 0\right)$. This observation however requires a more thorough investigation with ultra high-speed imaging in a combined topview and side-view with an orthogonal microscope [36, 37].

A few comments on the applicability of these results for the smaller nanodroplets are in order. The proposed models of heat transfer and rectified heat transfer are only valid for micron-sized droplets. When the size of the droplet approaches the thermal diffusion length of around $40 \mathrm{~nm}$, Eq. 5.3, the thin thermal boundary layer assumption, Eq. 5.4, can no longer be applied. The best modeling results will be achieved starting from radii 100 times larger than the thermal diffusion length. The limitations of this model for nanodroplets can be overcome by developing a numerical code taking into account the temperature distribution around the vapor nucleus and to couple these to the other physical effects, i.e. phase transition, inertia, and heat transfer. Nevertheless, the proposed theoretical models give us valuable knowledge about the use of ADV for drug delivery. Moreover, it can be applied to different ambient temperatures as well as to different PFCs, such as perfluorobutane $\left(\mathrm{T}_{\mathrm{b}}=\right.$ $\left.-1.7^{\circ} \mathrm{C}\right)$ and perfluorohexane $\left(\mathrm{T}_{b}=+56^{\circ} \mathrm{C}\right)$. For instance, following Eq. 5.7 perfluorobutane will vaporize more violently than perfluoropentane at the same ambient temperature, at $\mathrm{T}_{\infty}=37^{\circ} \mathrm{C}$ in fact five times as fast.

Let us now consider the prediction of the speed of the vapor bubble expansion. If a more gentle expansion rate of the droplets is desired, a manipulation of the heat diffusivity is required to limit the heat transfer processes. And secondly, the application of ultrasound while the vaporization is still in progress will lead to a more rapid vapor bubble growth due to rectified heat transfer 
phenomena and its associated cycles of vaporization and condensation may cause mechanical stress to neighboring cells and tissues, which may be favorable for the local uptake of drugs. The inclusion of rectified heat transfer phenomena, see Eq. 5.15, adds an intrinsic component $\Delta R \sqrt{3} \arctan (R / \Delta R \sqrt{3})$ on top of the heat transfer expression introduced in Eq. 5.7. The small perturbation $\Delta R$ is a function of the ultrasound driving pressure $P_{A}$ and, through its resonant behavior, of its frequency $f$. Thus the rectified heat transfer can be controlled by tuning these parameters. In this study we kept $P_{A}$ and $f$ fixed and $\Delta R$ was measured directly from the $R-t$ curves. Theoretically, $\Delta R$ should also be a function of the vapor bubble radius, however for simplicity we considered it to be constant. From experiment we also conclude that the change in $\Delta \mathrm{R}$ was not significant. It is also clear that a large number of cycles may promote the effects of rectified heat transfer. The role of the number of cycles with respect to the combined effect of nucleation and vaporization needs further investigation. We have shown that with our choice of driving frequency, pressure amplitudes, and ambient temperatures, it is sufficient to initiate the vaporization with only 1 to 4 cycles of ultrasound. It should also be mentioned that the approach of the condensation of the vapor bubbles by exerting them with sufficiently high ambient pressure was used to generate droplets [38]. In our current experiments and at the present ambient temperatures, we never observed a complete re-condensation of the vapor bubbles; once it initiates, a vapor nucleus will always end up as a vapor bubble and never as a droplet.

Finally, it was observed that the expansion rates of droplets containing nanoemulsions, for instance oil-in-PFP-in-water, is smaller than those of single phase droplets, see Fig. 5.10 for a typical multiphase droplet vaporization event. One can apply similar considerations of the heat transfer to develop a theory for expansion rates of droplets containing nanoemulsions inside. However this will require multiple free parameters to incorporate the size distribution and the concentration of the emulsion, as these quantities are not known a priori and may vary from droplet to droplet. It remains a task for future work to extend this theoretical framework to nanoemulsion droplets, which are of great importance for ultrasound-triggered drug delivery applications. 


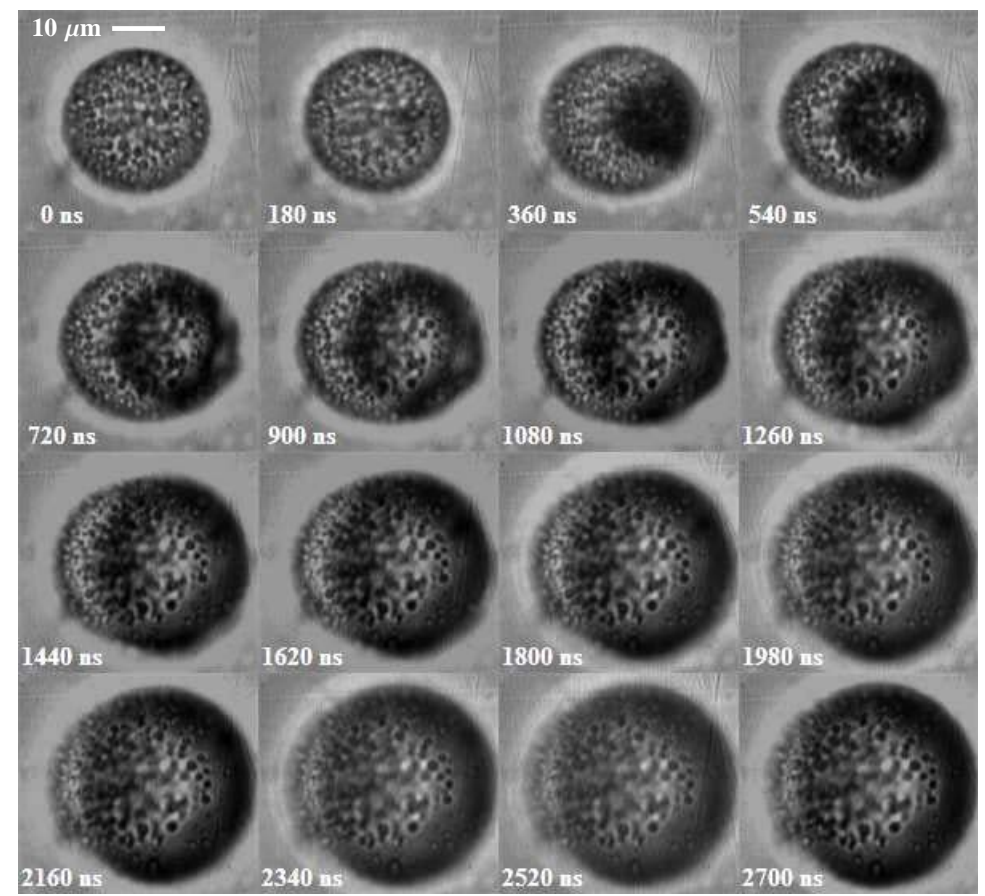

Figure 5.10: A set of images showing acoustic droplet vaporization of a 14.5- $\mu \mathrm{m}$ radius oil-in-PFP-in-water microdroplet taken at $5.5 \mathrm{Mfps}$ at an ambient temperature of $38^{\circ} \mathrm{C}$. The expansion rate is smaller than that of the single phase PFP-in-water microdroplet of Fig. 5.2. 


\subsection{Conclusion}

Acoustic microdroplet vaporization was imaged for the first time at a nanoseconds time scale with a sampling rate of 3-5 images per cycle, being able to capture prenucleation, nucleation, US and post US, all for a single vaporization event. With the proposed method of pressure restoration it was shown that the inception of nucleation occurs during the peak negative half cycle of ultrasound and slightly shifted towards the end of it. Following nucleation, the physics of the vapor bubble expansion is shown to be merely heat transfer limited. The vapor bubble expansion was observed to have two components, one monotonic with a typical velocity of expansion of $5-10 \mathrm{~m} / \mathrm{s}$ and a second oscillating component with the typical amplitude of $1.2 \mu \mathrm{m}$ associated with the applied ultrasound. The vapor bubble growth is increased by acoustic activation and through a simple physical model it was shown to be result of rectified heat transfer when the ultrasound is ON.

\section{References}

[1] O. D. Kripfgans, J. B. Fowlkes, D. L. Miller, O. P. Eldevik, and P. L. Carson, "Acoustic droplet vaporization for therapeutic and diagnostic applications”, Ultrasound Med. Biol. 26, 1177-1189 (2000).

[2] D. R. Evans, D. F. Parsons, and V. S. J. Craig, "Physical properties of phase-change emulsions", Langmuir 22, 9538-9545 (2006).

[3] O. D. Kripfgans, C. Orifici, P. Carson, K. A. Ives, O. P. Eldevik, and J. B. Fowlkes, "Acoustic droplet vaporization for temporal and spatial control of tissue occlusion: a kidney study", IEEE Trans. Ultrason. Ferroelec. Freq. Contr. 52, 1101-1110 (2005).

[4] M. Zhang, M. L. Fabiilli, K. J. Haworth, F. Padilla, S. D. Swanson, O. D. Kripfgans, P. Carson, and J. Fowlkes, "Acoustic droplet vaporization for enhancement of thermal ablation by high intensity focused ultrasound", Acad. Radiol. 18, 1123-1132 (2011).

[5] P. S. Sheeran, V. P. Wong, S. Luois, R. J. McFarland, W. D. Ross, S. Feingold, T. Matsunaga, and P. Dayton, "Decafluorobutane as a phase-change contrast agent for low-energy extravascular ultrasonic imaging.", Ultrasound Med. Biol. 37, 1518-1530 (2011). 
[6] P. Sheeran and P. Dayton, "Phase-change contrast agents for imaging and therapy.", Curr. Pharm. Design 18, 2152-2165 (2012).

[7] N. Rapoport, A. Kennedy, J. Shea, C. Scaife, and K.-H. Nam, "Controlled and targeted tumor chemotherapy by ultrasound-activated nanoemulsions/microbubbles.”, J. Contr. Rel. 138, 268-276 (2009).

[8] C. Wang, S. Kang, Y. Lee, Y. Luo, Y. Huang, and C. Yeh, "Aptamerconjugated and drug-loaded acoustic droplets for ultrasound theranosis.", Biomaterials 33, 1939-1947 (2012).

[9] M. Fabiilli, K. Haworth, I. Sebastian, O. Kripfgans, P. Carson, and J. Fowlkes, "Delivery of chlorambucil using an acoustically-triggered perfluoropentane emulsion.”, Ultrasound Med. Biol. 36, 1364-1375 (2010).

[10] J. Riess, "Oxygen carriers (blood substitutes) - raison d'etre, chemistry, and some physiology", Chemical Reviews 101, 2797-2919 (2001).

[11] O. Couture, M. Faivre, N. Pannacci, A. Babataheri, V. Servois, P. Tabeling, and M. Tanter, "Ultrasound internal tattooing", Med. Phys. 38, 1116-1123 (2011).

[12] N. Y. Rapoport, Z. Gao, and A. Kennedy, "Multifunctional nanoparticles for combining ultrasonic tumor imaging and targeted chemotherapy", J. Natl. Cancer Inst. 99, 1095-1106 (2007).

[13] P. Mohan and N. Rapoport, "Doxorubicin as a molecular nanotheranostic agent: Effect of doxorubicin encapsulation in micelles or nanoemulsions on the ultrasound-mediated intracellular delivery and nuclear trafficking.", Mol. Pharmaceut. 7, 1959-1973 (2010).

[14] O. Kripfgans, M. Fabiilli, P. Carson, and J. Fowlkes, "On the acoustic vaporization of micrometer-sized droplets", J. Acoust. Soc. Am. 116, 272-281 (2004).

[15] Z. Wong, O. Kripfgans, A. Qamar, J. Fowlkes, and J. L. Bull, "Bubble evolution in acoustic droplet vaporization at physiological temperature via ultra-high speed imaging", Soft Matter 7, 4009-4016 (2011). 
[16] S. Kang and C. Yeh, "Intracellular acoustic droplet vaporization in a single peritoneal macrophage for drug delivery applications", Langmuir 27, 13183-13188 (2011).

[17] N. Reznik, R. Williams, , and P. N. Burns, "Investigation of vaporized submicron perfluorocarbon droplets as an ultrasound contrast agent", Ultrasound Med. Biol. 37, 1271-1279 (2011).

[18] A. Qamar, Z. Z. Wong, J. B. Fowlkes, and J. L. Bull, "Dynamics of acoustic droplet vaporization in gas embolotherapy", Appl. Phys. Lett. 96, 143702 (2010).

[19] K. J. Haworth, O. Kripfgans, and J. L. Bull, "Initial growth and coalescence of acoustically vaporized perfluorocarbon microdroplets", IEEE International Ultrasonics Symposium (2008).

[20] M. Fabiilli, K. Haworth, N. Fakhri, O. Kripfgans, P. Carson, and J. Fowlkes, "The role of inertial cavitation in acoustic droplet vaporization.”, IEEE Trans. Ultrason. Ferroelec. Freq. Contr. 56, 1006-1017 (2009).

[21] M. Fabiilli, J. Lee, O. Kripfgans, P. Carson, and J. Fowlkes, "Delivery of water-soluble drugs using acoustically triggered perfluorocarbon double emulsions", Pharm. Res. 27, 2753-2765 (2010).

[22] C. T. Chin, C. Lancée, J. Borsboom, F. Mastik, M. E. Frijlink, N. de Jong, M. Versluis, and D. Lohse, "Brandaris 128: A digital 25 million frames per second camera with 128 highly sensitive frames", Rev. Sci. Instr. 74, 5026-5034 (2003).

[23] E. Gelderblom, H. J. Vos, F. Mastik, T. Faez, T. Kokhuis, A. F. W. van der Steen, N. de Jong, D. Lohse, and M. Versluis, "Brandaris 128 ultra-high-speed imaging facility: 10 years of operation, updates and enhanced features”, Rev. Sci. Instr. 83, 103706 (2012).

[24] S. M. van der Meer, B. Dollet, C. T. Chin, A. Bouakaz, M. Voormolen, N. de Jong, M. Versluis, and D. Lohse, "Microbubble spectroscopy of ultrasound contrast agents", J. Acoust. Soc. Am. 121, 648-656 (2007).

[25] A. Pierce, Acoustics - An Introduction to its Physical Principles and Applications, 434-435 (McGraw-Hill Book Co., New York) (1981). 
[26] C. Lee, A. Anilkumar, and T.G.Wang, "Static shape and instability of an acoustic levitated drop", Phys. Fluids A 3, 2497-2516 (1991).

[27] T. Leighton, The Acoustic Bubble, chapter 4 The Forced Bubble, 302303 (Academic Press, London, UK) (1994).

[28] "Collection of critically evaluated thermodynamic property data", http://wtt-pro.nist.gov (date last viewed 10/29/12).

[29] M. Plesset and S. Zwick, "A nonsteady heat diffusion problem with spherical symmetry”, J. Appl. Phys. 23, 95-98 (1952).

[30] M. Plesset and S. Zwick, "The growth of vapor bubbles in superheated liquid”, J. Appl. Phys. 25, 493-500 (1954).

[31] H. Forster and N. Zuber, "Growth of vapor bubbles in superheated liquid", J. Appl. Phys. 25, 474-478 (1954).

[32] G. Trammell, "Sound waves in water containing vapor bubbles", J. Appl. Phys. 33, 1662-1670 (1962).

[33] R. Finch and E. Neppiras, "Vapor bubble dynamics", J. Acoust. Soc. Am. 53, 1402-1410 (1973).

[34] T. Wang, "Rectified heat transfer", J. Acoust. Soc. Am. 56, 1131-1143 (1974).

[35] Y. Hao and A. Prosperetti, "The dynamic of vapor bubbles in acoustic pressure fields", Phys. Fluids 11, 2008-2019 (1999).

[36] H. J. Vos, B. Dollet, J. G. Bosch, M. Versluis, and N. de Jong, "Nonspherical vibrations of microbubbles in contact with a wall-a pilot study at low mechanical index", Ultrasound Med. Biol. 34, 685-688 (2008).

[37] H. J. Vos, B. Dollet, M. Versluis, and N. de Jong, "Nonspherical shape oscillations of coated microbubbles in contact with a wall", Ultrasound Med. Biol. 37, 935-948 (2011).

[38] P. S. Sheeran, S. Luois, P. A. Dayton, and T. O. Matsunaga, "Formulation and acoustic studies of a new phase-shift agent for diagnostic and therapeutic ultrasound", Langmuir 27, 10412-10420 (2011). 


\section{6 The role of $\mathrm{gas}^{\ddagger *}$}

In the present chapter we study both experimentally and theoretically the dynamics of an ultrasound driven vapor bubble of perfluoropentane (PFP) inside a droplet of the same liquid, immersed in a water medium superheated respect to the PFP boiling point. We determine the temporal evolution of the bubble radius with ultra-high speed imaging at 20 million frames per second. In addition we model the vapor-gas bubble dynamics, based on a RayleighPlesset-type equation, including thermal and gas diffusion inside the liquid. We compare the numerical results with the experimental data and find good agreement. We underline the fundamental role of gas diffusion in order to prevent total recondensation of the bubble at collapse.

\subsection{Introduction}

Current chemotherapeutical cancer treatment has severe side effects caused by the adverse outcome of the drugs on healthy tissue. Therefore it is de-

\footnotetext{
$\ddagger$ Published as: Oleksandr Shpak, Laura Stricker, Michel Versluis, and Detlef Lohse, "The role of gas in ultrasonically driven vapor bubble growth", Physics in Medicine and Biology 58 (8). 2523-2535 (2013).

${ }^{*}$ O. Shpak contributed to the analysis, experiments, writing and discussions. The numerical simulations are done by L. Stricker
} 
sirable to create drug delivery agents, which will release the drug locallly at the tumor site. Recent studies have shown that liquid emulsion phase-change droplets composed of perfluorocarbons (PFC) such as perfluoropentane (PFP) have the potential to be a highly efficient system for local drug delivery $[1,2]$. PFP liquid has a boiling temperature of $29^{\circ} \mathrm{C}$. However, when stabilized the submicron- and micron-sized PFP droplets do not spontaneously vaporize when injected in vivo at $37^{\circ} \mathrm{C}$ temperature $[3,4]$. A phase transition can be triggered by application of an acoustic pusle of sufficiently high pressure $[3,5]$. An explosive evaporation takes place and the drug is released from the liquid core $[6,7]$. The advantage of the so-called acoustic droplet vaporization (ADV) technique are its non-invasive and inexpensive character. Moreover, the resulting bubbles become acoustically active ultrasound contrast agents, which are detectable for at least one microsecond after nucleation [8]. Although the success of this approach for localized drug delivery has been demonstrated [1], the physical mechanisms underlying the ADV process have not been well described until now [9].

In this Chapter we use ultra-high speed optical imaging to study experimentally the ultrasound driven vapor bubble growth in the superheated liquid at nanoseconds timescale. We capture the vaporization dynamics of a single emulsion system: PFP droplets of a radius of $5 \mu \mathrm{m}$ immersed in water. This system is simpler to model and to track experimentally with respect to multiple phase emulsions used for localized drug delivery $[6,10]$. Some theoretical work has already been done on the modelling of acoustic droplet vaporization at microseconds time scale, in the frame of gas embolotherapy, a promising cancer treatment involving tumor occlusion with gas microbubbles selectively formed from the liquid droplets [11]. Here we focus on the nanoseconds time scale in order to capture the complex interaction of the ultrasound with the vapor bubble. We develop a PDE model based on a Rayleigh-Plesset-type equation, including thermal and gas diffusion inside the PFP liquid. We compare the experimental data with the numerical results, finding a good agreement. Such a model can therefore provide a valuable instrument for practical purposes, where one wants to control the size of resulting bubbles as a function of time, for example when they are used as ultrasound contrast agents [8]. We underline the fundamental role of gas diffusion, in order to prevent total recondensation of the bubble at collapse, provided that the periodic ultrasound driving is present. Recondensation of a vapor bubble was thought as a possibility to induce reiterated phase transition of the same droplet. For applications such as sonoporation and tumor imaging this possibility may be 
of a crucial importance in order to avoid repeated injections of ADV agents.

Mathematical models for spherically symmetric vapor-gas bubbles have already been formulated in Refs. [12-18], and their small oscillations have been analytically investigated in detail in Refs. [13, 18]. In the present paper we will show that our approach has two great advantages with respect to the earlier models. First, it is simpler, as it does not include diffusion of the gas inside the vapor, though still able to capture the relevant experimental features. And secondly, it does not present fitting parameters such as the accommodation coefficient for the description of the evaporative process [12, 13].

\subsection{Materials and experimental methods}

The PFP droplet emulsion was prepared with bovine serum albumin using the same method adopted in Refs.[19, 20]. The resulting highly diluted suspension of droplets was injected into an OptiCell (Thermo Fisher Scientific, Waltham, MA, USA) in order to observe the droplets individually. The OptiCell was submerged in a 1 liter temperature-controlled water tank. A schematic of the experimental setup is shown in Figure 6.1. Microdroplets were phasetransitioned with a focused 1.5-inch diameter single-element transducer (A381S; Olympus Panametrics, Antwerpen, Belgium, 3.5 MHz center frequency, NA 1.0). The acoustic driving pulse was generated by an arbitrary waveform generator (Tabor 8026; Tabor Electronics, Tel Hanan, Israel) amplified by a RF-amplifier (ENI 350L, Electronic Navigation Industries, Inc., Rochester, NY, USA). Droplet samples were vaporized with a single ultrasound pulse, consisting of a burst of 6 cycles and a driving pressure amplitude of $P_{a}$ of 4.5 $\mathrm{MPa}$. The pressure value was calibrated in the same setup at the position of the droplets using a $0.2 \mathrm{~mm}$ PVDF probe hydrophone (DC27/000658, Precision Acoustics Ltd., Dorchester, UK). An Olympus microscope (Olympus; Zoeterwoude, The Netherlands) with a 40x water immersed objective (NA = 0.7) was focused on an OptiCell providing a spatial resolution of $0.269 \mu \mathrm{m}$ per pixel. The radial response of the insonified droplets was recorded with an ultra-fast recording camera, the Brandaris 128 (Chin et al. 2003). The camera was set to record 6 movies of 128 frames in a single run at a frame rate up to 20 million frames per second (fps), thus providing an interframe time as low as 50 nanoseconds. The first recording was always done without ultrasound to estimate the noise level of the system. The region of interest was 


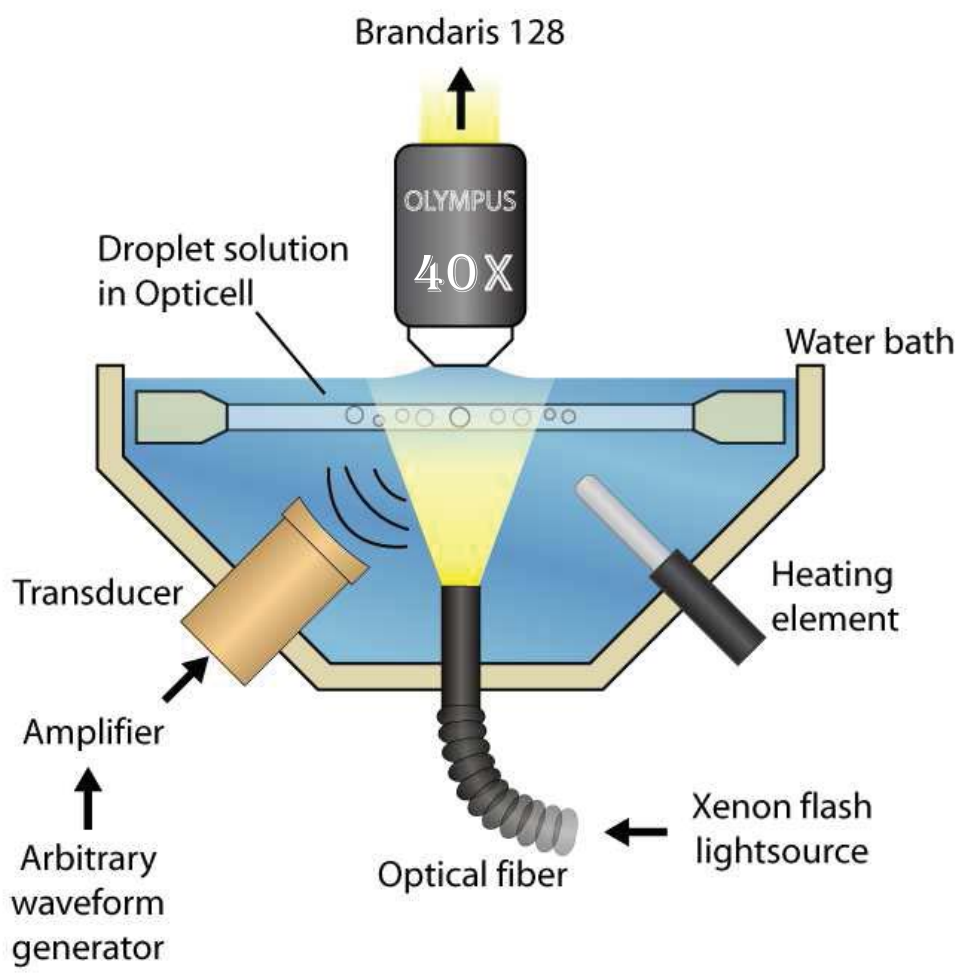

Figure 6.1: Schematic view of the experimental setup. An OptiCell polystyrene container with the microdroplet suspension was positioned inside a water tank kept to a constant temperature $\mathrm{T}_{\infty}$, superheated with respect to the PFP boiling point. A focused transducer sent the US-pulses from a side with an inclination of $45^{\circ}$ with respect to the plane of the Opticell. The samples were imaged from the top with an upright microscope coupled to the Brandaris 128 high-speed camera and irradiated from below with a Xenon flash light. 
illuminated with a $65 \mathrm{~mJ}$ Xenon flash pulse (30 $\mu$ s duration) through the light guide (SCHOTT AG, Mainz, Germany) from below. From the movies, the radii $R(t)$ of the resulting droplets as a function of time were measured using custom-designed image analysis software based on a minimum cost function. The droplet radius was calculated as the mean distance from the centroid to the edge of the droplet in a radial coordinate system averaged over the full $2 \pi$ angle. The vapor bubble dynamics model used here (see Sect. 3) assumes spherical symmetry of the problem. However, due to the homogeneous bubble nucleation nature of ADV, the vapor nucleus appears not necessarily in the center of the droplet. In order to be able to compare the numerical results with experimental data, we chose a case in which the nucleation occured well centered within the droplet.

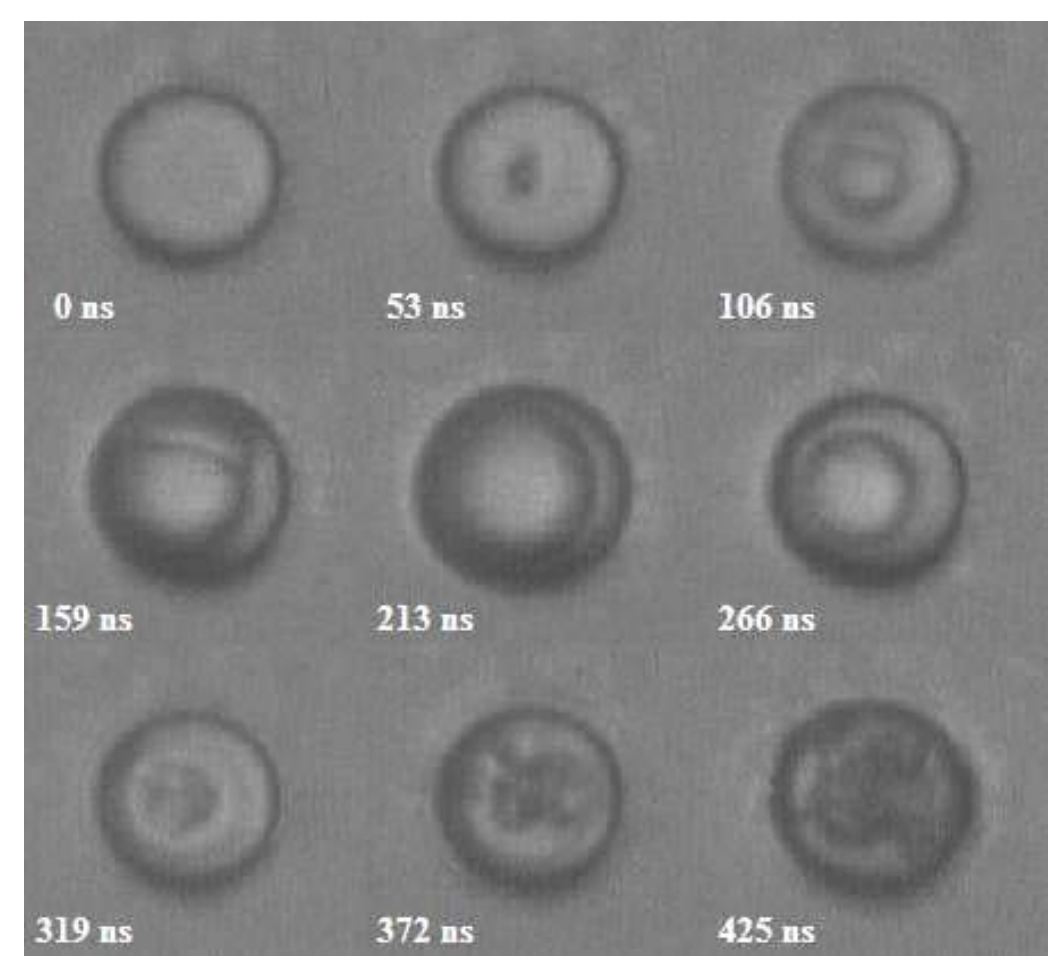

Figure 6.2: A set of consecutive images showing acoustic droplet vaporization at $15^{\circ} \mathrm{C}$ superheat, taken at a frame rate of $18.81 \mathrm{Mfps}$. Driving parameters: frequency $f=3.5 \mathrm{MHz}$, pressure $\mathrm{Pa}=4.5 \mathrm{MPa}$. The initial radius of the droplet is $\mathrm{R}_{0}=5.4 \mu \mathrm{m}$. The vapor bubble inside the droplet undergoes vaporization/(re)condensation cycles with the frequency of the applied ultrasound. 


\subsection{Model}

In the model we consider a spherical bubble of PFP inside an infinite medium of liquid PFP, neglecting the surrounding water. We consider both the vapor and the gas inside the bubble and we model their dynamics independently, i.e. we neglect the diffusion of gas inside the vapor in the bubble. Both the gas and the vapor inside the bubble are treated as perfect gases in saturated conditions. For a complete description of the vapor dynamics and the evolution of the thermal field, we refer the reader to Refs.[21, 22] and Ref. [23] respectively. We assume a spatially uniform pressure inside the bubble, low Mach number regimes, spherical symmetry and shape stability. The gas considered in the simulations is air with standard composition.

The radial dynamics of the bubble is described by a Rayleigh-Plesset-type equation with the formulation used in Ref. [23]:

$$
\left(1-\frac{\dot{R}}{c_{L}}\right) R \ddot{R}+\frac{3}{2}\left(1-\frac{\dot{R}}{3 c_{L}}\right) \dot{R}^{2}=\frac{1}{\rho_{L}}\left(1+\frac{\dot{R}}{c_{L}}+\frac{R}{c_{L}} \frac{d}{d t}\right)\left[p_{B}-p_{A}\right],
$$

in which $R$ is the bubble radius, $\rho_{\mathrm{L}}$ is the density of the liquid, $c_{\mathrm{L}}$ is the speed of sound and the dot is used to indicate time derivatives. The ambient pressure in the liquid, $p_{A}$, is calculated as

$$
p_{A}=p_{\infty}-P_{a} \cos \omega t
$$

with $P_{a}$ the acoustic driving pressure, $f=\omega / 2 \pi$ the driving frequency and $p_{\infty}$ the static pressure. Such a phase of the driving pressure has been taken because the bubble nucleation is most likely to occur at the negative pressure peak, when the stretching of the liquid is maximal [20]. The pressure at the bubble surface on the liquid side, $p_{\mathrm{B}}$, is derived from the normal stress balance at the bubble wall [23]

$$
p=p_{B}+4 \mu_{L} \frac{\dot{R}}{R}+\frac{2 \sigma}{R},
$$

where $\mu_{\mathrm{L}}$ is the dynamic viscosity of the liquid and $\sigma$ is the surface tension. The total pressure inside the bubble, $p$, can be expressed as the sum of the partial pressures of gas $p_{G}$ and vapor $p_{V}$ :

$$
p=p_{V}+p_{G}
$$


The Clausius-Clapeyron relation holds [22]:

$$
\frac{d p_{V}}{d T_{S}}=\frac{L \rho_{V}}{T_{S}},
$$

where $L$ is the latent heat and $\rho_{V}$ the density of the vapor. An explicit expression for $p_{V}$ can be found by integrating Eq. (6.5) with the further use of the perfect gas law of the vapor [22]

$$
p_{V}=P_{0} \exp \left(-\frac{L}{R_{V} T_{S}}\right) \text {. }
$$

Here $P_{0}=p_{V, 0} \exp \left(L / R_{V} T_{0}\right)$ is a constant and $R_{V}$ is the universal gas constant divided by the molecular mass of the vapor. The pressure of the gas $p_{G}$ is related to the concentration of the gas in the liquid at the bubble wall, $\left.\mathrm{c}\right|_{\mathrm{R}}$, by Henry's law:

$$
\mathrm{p}_{\mathrm{G}}=\left.\mathrm{k}_{\mathrm{H}} \mathrm{c}\right|_{\mathrm{R}}
$$

where $k_{H}$ is the Henry's constant, derived from air solubility data inside PFP.

We assume a uniform temperature field inside the bubble. This is a common assumption for vapor bubbles and its validity has been verified in Ref. [21]. In order to describe the evolution of the temperature field in the liquid $\mathrm{T}(\mathrm{r}, \mathrm{t})$ we use the standard convection-diffusion equation [23]:

$$
\rho_{\mathrm{L}} \mathcal{C}_{\mathrm{p}, \mathrm{L}}\left(\frac{\partial \mathrm{T}}{\partial \mathrm{t}}+\frac{\mathrm{R}^{2} \dot{\mathrm{R}}}{\mathrm{r}^{2}} \frac{\partial \mathrm{T}}{\partial \mathrm{r}}\right)=\mathrm{k}_{\mathrm{L}} \nabla^{2} \mathrm{~T}
$$

where $r$ is the radial coordinate measured from the bubble center, while $c_{p, L}$ and $k_{L}$ are the specific heat and thermal conductivity of the liquid. Far from the bubble, the liquid temperature is assumed to remain undisturbed at the initial value $T_{\infty}$ and the following boundary condition is adopted:

$$
\frac{\partial \mathrm{T}}{\partial r}(\infty, \mathrm{t})=0 \text {. }
$$

At the bubble wall, the temperature is continuous. Therefore:

$$
T(R(t), t)=T_{S}(t),
$$

i.e. $T_{S}$ - the temperature at the surface - equals the temperature inside the bubble. The energy balance at the bubble wall can be written as [21]:

$$
L \frac{d}{d t}\left(\rho_{V} V\right)+\rho_{V} V c_{s} \frac{d T_{S}}{d t}=\left.4 \pi R^{2} k_{L} \frac{\partial T}{\partial r}\right|_{R},
$$


with $c_{S}=c_{P, V}-L / T_{S}$ the specific heat of the saturated vapor, and $V$ the volume of the bubble. The first term in Eq. (6.11) is the heat employed to evaporate the liquid, the second is the change of vapor enthalpy in time due to temperature changes and the third one is the heat transferred by conduction from the liquid to the bubble. The change of gas enthalpy in time due to temperature changes has been neglected as we assumed $\rho_{\mathrm{G}}<<\rho_{\mathrm{V}}$. This assumption turned out to be justified because we found $\rho_{\mathrm{G}} / \rho_{\mathrm{V}} \sim 10^{-2}$, as will be shown later. We also neglected the heat flux by diffusion inside the bubble because of the assumption of uniform temperature. By making use of vapor mass conservation, the Clausius Clapeyron equation and the perfect gas law for the vapor, Eq. (6.11) can be rewritten as

$$
\left(c_{P}+\frac{L^{2}}{R_{V} T_{S}^{2}}-\frac{2 L}{T_{S}}\right) \frac{d T_{S}}{d t}=\frac{3}{\rho_{V} R}\left(\left.k_{L} \frac{\partial T}{\partial r}\right|_{R}-\rho_{V} L \dot{R}\right)
$$

from which an explicit expression for $\frac{\mathrm{dT} S}{\mathrm{dt}}$ can be extracted. For a detailed derivation of Eq. (6.12) we refer the reader to Ref. [22].

Similarly to the temperature, the concentration of the gas in the liquid $c(r, t)$ is described by the standard convection-diffusion equation:

$$
\frac{\partial c}{\partial t}+\frac{R^{2} \dot{R}}{r^{2}} \frac{\partial c}{\partial r}=D_{G} \nabla^{2} c .
$$

Here $D_{G}$ is the mass diffusivity of the gas inside the liquid, calculated by means of the Stokes-Einstein formula [24]: $D_{G}=k_{B} T /\left(6 \pi \mu_{L} r_{G}\right)$, where $k_{B}$ is the Boltzmann's constant and $r_{G}$ is the gas molecular radius calculated as half of the collisional diameter, following Ref. [25]. Far from the bubble, the gas concentration is assumed to remain undisturbed at the initial value $c_{\infty}=0.68 \mathrm{~kg} / \mathrm{m}^{3}$, equal to the solubility of air inside PFP [26], and obviously, as a further boundary condition:

$$
\frac{\partial c}{\partial r}(\infty, t)=0
$$

Mass conservation of the gas implies:

$$
\frac{d}{d t}\left(\rho_{G} V\right)=\left.4 \pi R^{2} D_{G} \frac{\partial c}{\partial r}\right|_{R},
$$

where $\rho_{G}$ is the gas density inside the bubble, related to the gas pressure by perfect gas law

$$
\frac{p_{G}}{\rho_{G}}=R_{G} T_{s}
$$


with $R_{G}$ the universal gas constant divided by the molecular weight of the gas. All the properties of PFP were taken from Refs. [26, 27].

For the solution of both the energy equation Eq. (6.8) and the gas diffusion equation Eq. (6.13) we used the method presented in Ref. [23], i.e. a pseudo-spectral collocation method. The reader is referred to that reference for details. We adopted the coordinate transformation [23]

$$
\frac{1}{\xi}=1+\frac{r / R(t)-1}{l},
$$

in order to map the semi-infinite range $R(t) \leqslant r<\infty$ into the finite range $1 \geqslant \xi \geqslant 0$. In (6.17), the constant $l$ is related to the thermal diffusion length in the liquid, as thermal diffusion is the dominating process in the observed phenomenon and it has been taken as [23]

$$
l=\ell \frac{\sqrt{D_{L} / \omega}}{R_{0}},
$$

where $D_{L}=k_{L} / c_{p, L} \rho_{L}$ is the liquid thermal diffusivity and $\ell$ is a constant number. We adopted a value of $\ell=20$, in agreement with Refs. [23]. We reformulated the energy equation and the gas diffusion equation by substituting the new variable $\xi$ inside the Eqs. (6.8) and (6.13), and we expanded the liquid temperature and the gas concentration fields in truncated Chebyshev series:

$$
\begin{aligned}
& \frac{\mathrm{T}(\mathrm{t}, \xi)}{\mathrm{T}_{\infty}} \approx \sum_{\mathrm{k}=0}^{\mathrm{N}} \mathrm{a}_{\mathrm{k}}(\mathrm{t}) \mathrm{T}_{2 \mathrm{k}}(\xi), \\
& \frac{\mathrm{c}(\mathrm{t}, \xi)}{\mathrm{c}_{\infty}} \approx \sum_{\mathrm{k}=0}^{\mathrm{N}} \mathrm{b}_{\mathrm{k}}(\mathrm{t}) \mathrm{T}_{2 \mathrm{k}}(\xi),
\end{aligned}
$$

in which $T_{2 k}(\xi)$ are the even Chebyshev polynomials. The coupled equations for the various $a_{k}(t)$ and $b_{k}(t)$ arising upon substitution of Eqs.(6.19) and (6.20) into Eqs. (6.8) and (6.13) were solved by collocation. Convergence was reached by using 45 terms in the Chebyshev expansions with 45 Gauss-Lobatto collocation points. In the limiting cases of the absence of gas diffusion, we have verified that the code developed for this work gave the same results as those used in Refs. [21] and [28] respectively, which had been both validated against analytical solutions. In the case of pure gas diffusion and no driving an additional validation was performed against experimental data [28]. 


\subsection{Results}

In experiments we measured the external radius of the droplet as a function of time. The statistical error of the measurement of the droplet radii was $150 \mathrm{~nm}$. The error was estimated from a measurement of the resting radius of the droplet (128 data points) before ultrasound was sent and amounts to twice the standard deviation of the droplet radius. Assuming incompressibility for liquid PFP, we extracted the radius $R(t)$ of the bubble, using mass conservation: $\rho_{L}\left(V_{d}(t)-V(t)\right)+\rho_{V} V(t)=\rho_{L} V_{d, 0}$, where $V_{d}(t)$ and $V(t)$ are the volume of the droplet and the volume of the bubble, respectively, $V_{d, 0}$ is the initial volume of the droplet, before the bubble nucleates, and $\rho_{\mathrm{L}}$ is the density of liquid PFP. Therefore

$$
R(t)=\sqrt[3]{\frac{\rho_{L}}{\rho_{L}-\rho_{V}}\left(R_{d}^{3}(t)-R_{d, 0}^{3}\right)}
$$

with $R_{d, 0}$ the initial radius of the droplet before the bubble nucleates and $R_{d}(t)$ the measured radius of the droplet. In Fig. 6.3 we compare the time evolution of the bubble radius extracted from the experimental data with simulations. We chose a case with one well isolated bubble remaining almost spherical and far from the droplet wall (see Fig. 6.2). A $4.5 \mathrm{MPa}$ pressure driving pulse with a frequency of $3.5 \mathrm{MHz}$ for the duration of six cycles was applied. The measurements of the driving pressure amplitude performed with the hydrophone were accurate within an absolute error of up to $200 \mathrm{kPa}$, based on the standard deviation of the acoustic pressure amplitudes required to activate droplets of the same size [29]. We took as initial radius $R_{0}$ for the simulations the lowest value inferred from experiments, which is $1 \mu \mathrm{m}$. The water surrounding the droplet was kept at the constant temperature $\mathrm{T}_{\infty}=44^{\circ} \mathrm{C}$, i.e. with a superheat of $15^{\circ} \mathrm{C}$ with respect to the boiling point of $\mathrm{PFP} \mathrm{T}_{\mathrm{b}}=29^{\circ} \mathrm{C}$. The error of the temperature measurements was estimated to be $1^{\circ} \mathrm{C}$, due to the circulation of water above the heating element. As the nucleation of the vapor bubble is an abrupt phenomenon, ideally we should consider as initial condition a constant temperature field equal to the water temperature, in the whole liquid PFP and the boiling temperature of PFP at the bubble wall. For numerical reasons, we smeared out this step condition and we took instead an initial temperature field $T_{0}(r)$ as

$$
\mathrm{T}_{0}(\mathrm{r})=\mathrm{T}_{\infty}-\left(\mathrm{T}_{\infty}-\mathrm{T}_{\mathrm{b}}\right) \exp \left(-\frac{\mathrm{r}-\mathrm{R}_{0}}{\delta-\mathrm{R}_{0}}\right)
$$


where $\delta-R_{0}$ describes the thickness of the thermal boundary layer. We tried different values for $\delta$, in the range $R_{0}<\delta \leqslant 2 R_{0}$, and we found that, as long as the initial boundary layer is restrained to one radius distance from the bubble wall, its effective thickness does not influence significantly the final result.

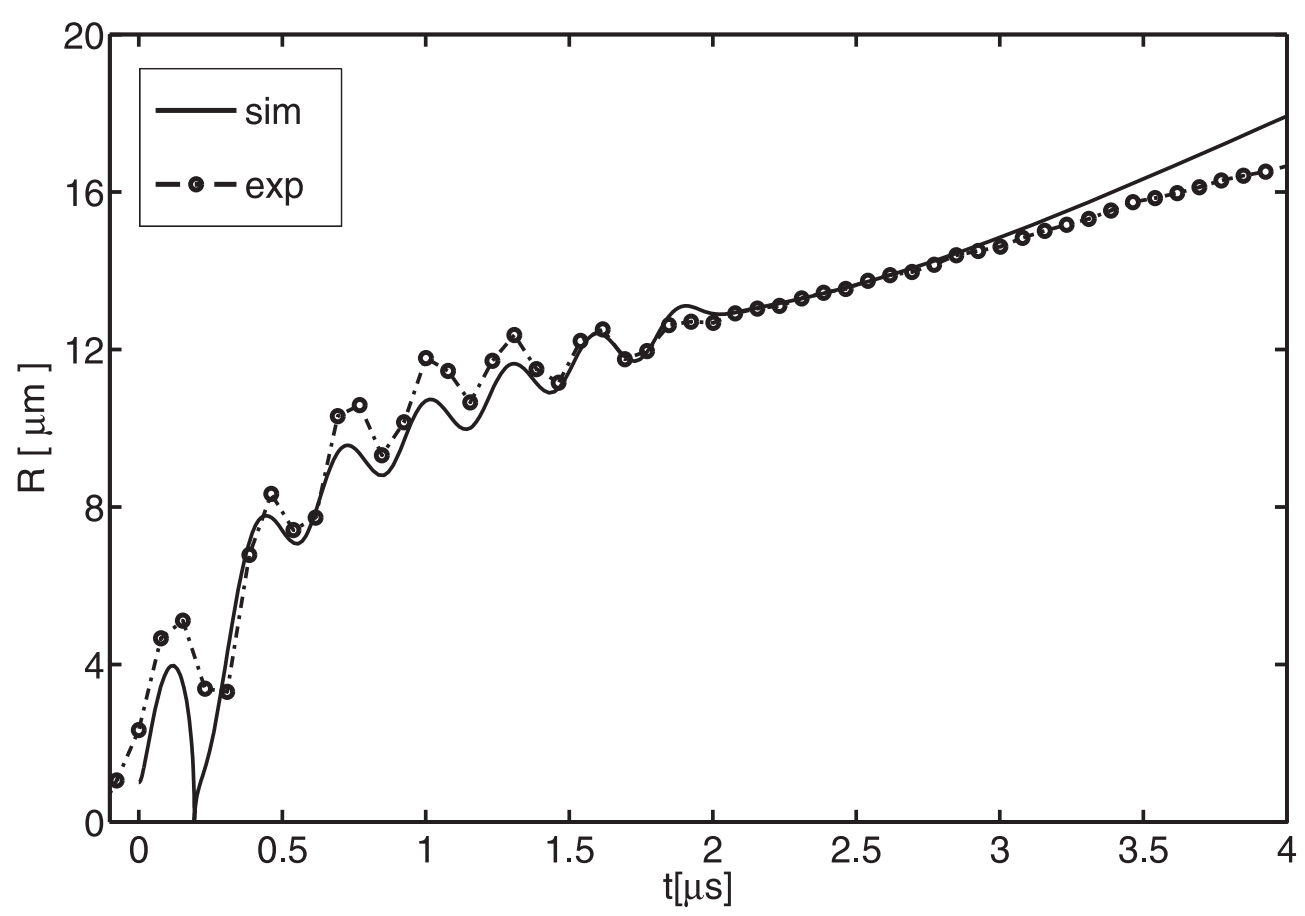

Figure 6.3: Radius-time curve $\mathrm{R}(\mathrm{t})$ of a PFP bubble from experiments (dash-dot) and simulations (solid) at $15^{\circ} \mathrm{C}$ superheating. Driving parameters: frequency $\mathrm{f}=$ $3.5 \mathrm{MHz}$, pressure $\mathrm{Pa}=4.5 \mathrm{MPa}$. After $2 \mu$ s the driving was turned off. The initial radius for simulations $R_{0}=1 \mu \mathrm{m}$ has been taken from the first value detected in experiments during the non-instantaneous nucleation phase.

Similarly, for the gas concentration inside the liquid, we assumed an initial distribution $\mathrm{c}_{0}(\mathrm{r})$, defined as

$$
c_{0}(r)=c_{\infty}-\left(c_{\infty}-c_{b}\right) \exp \left(-\frac{r-R_{0}}{\delta^{\prime}-R_{0}}\right)
$$

where $R_{0}<\delta^{\prime} \leqslant 2 R_{0}$ and $\left(\delta^{\prime}-R_{0}\right)$ describes the thickness of the mass diffusive boundary layer. Here $c_{\infty}=0.6846 \mathrm{~kg} / \mathrm{m}^{3}$ is the concentration of air inside PFP, given by the solubility of air inside PFP at $1 \mathrm{~atm}$, and $\mathrm{c}_{\mathrm{b}}$ is the 
initial concentration of gas at the bubble wall, set by Henry's law. We assumed the initial amount of gas trapped inside the bubble to be zero, therefore $c_{b}=0 \mathrm{~kg} / \mathrm{m}^{3}$. The same considerations reported for the initial temperature field apply also to the initial gas concentration field. At initial stages the discrepancies between experimental data and simulations may be attributed to the shape instabilities of the vapor bubble after the first rebounce. After the first collapse, such discrepancies, at each time instant, lie approximately within the $10 \%$ error of the experimental valuess. At later stages the vapor bubble approaches the boundary of a PFP droplet and the assumption of infinite PFP liquid is no longer valid.

The role of gas diffusion inside the bubble turns out to be of primary importance, provided acoustic forcing is present. Although the ratio of gas to vapor mass $m_{G} / m_{V}$ is of order $10^{-2}$, gas diffusion is necessary to avoid complete condensation of the bubble after the first collapse of the vapor bubble. Fig. 6.4 shows that, without including gas diffusion, the bubble does not survive the first driving cycle. Simulations with different values of $c_{\infty}$, down to $10 \%$ of the solubility of air in PFP, quantitatively showed the same behavior. Below $10 \%$, the gas pressure is not high enough to prevent recondensation of the bubble.

The radial dynamics of the vapor bubble is governed by thermal diffusion. Removing the acoustic forcing (dashed line), on the considered time scale, we retrieve the same growth rate found with the acoustic forcing (see Fig.6.4, dashed line). In this limiting case, including the gas diffusion did not have any significant effect on the bubble growth. With no ultrasound affecting the system, from the classical Plesset-Zwick theory [30], we expect a first phase with low growth rate due to surface tension (before $0.2 \mu \mathrm{s}$ in Fig.6.4), followed by a linear growth phase dominated by inertia and eventually a square-root growth dominated by diffusion. Considering the limiting case of purely inertial growth, with a uniform liquid temperature field equal to $T_{\infty}$, from the Rayleigh-Plesset-type equation, assuming $\ddot{R}=0$, it is possible to estimate the radial inertial expansion as: $R_{\text {in }} \sim t \sqrt{(2 / 3) \Delta p / \rho_{L}}$, where $\Delta p=p_{\infty}-p_{V, s a t}\left(T_{\infty}\right)$ with $p_{V, s a t}$ imposed by the Clausius-Clapeyron theorem as the pressure of the saturated vapor at $\mathrm{T}_{\infty}$. Considering the other limiting case of purely thermal diffusive growth with a uniform pressure field in the liquid equal to $p_{\infty}$, it is possible to estimate from the energy equation the radial thermal expansion as follows: $R_{t h} \sim k_{L} \Delta T \sqrt{t} /\left(L \rho_{V} \sqrt{D_{L}}\right)$ where $\Delta T=$ $\mathrm{T}_{\infty}-\mathrm{T}_{V, \text { sat }}\left(\mathrm{p}_{\infty}\right)$ with $\mathrm{T}_{V, \text { sat }}\left(\mathrm{p}_{\infty}\right)$ again imposed by the Clausius-Clapeyron theorem as the temperature of the saturated vapor at $p_{\infty}$ [30]. At a specific 


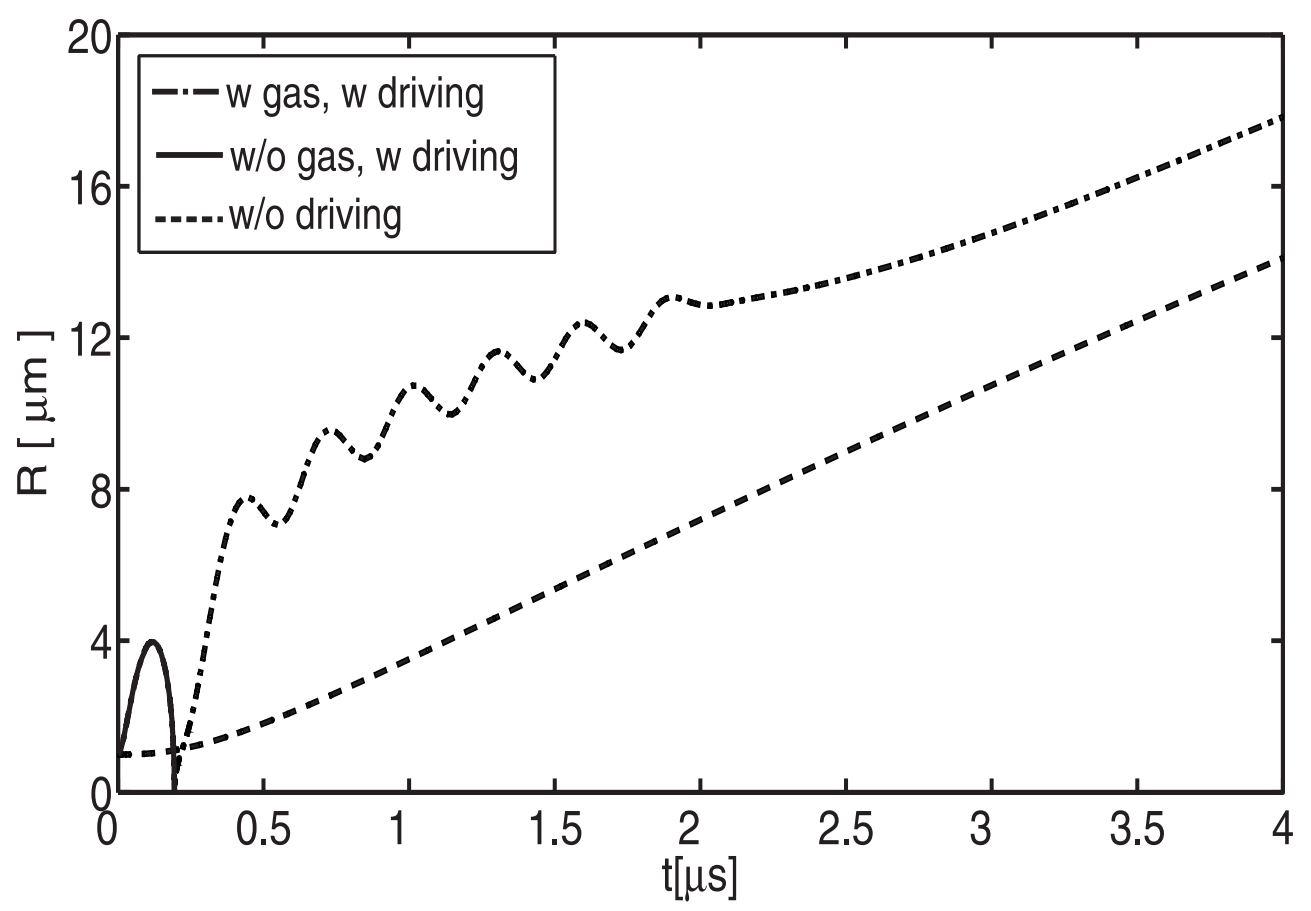

Figure 6.4: Radius time curve $\mathrm{R}(\mathrm{t})$ from simulations for a $1 \mu \mathrm{m}$ bubble in the same conditions of Fig.6.3 without (dash) and with driving, both with (dash-dot) and without (solid) gas diffusion. When the bubble is forced and without gas diffusion the bubble undergoes complete condensation after the first collapse. When the bubble is unforced, gas diffusion has no effect on the bubble dynamics. 
instant $\bar{t}$, the smallest value of either $R_{\text {th }}$ or $R_{\text {in }}$ is the one that governs the dynamics. At the specific time which we are considering $(\bar{t}=4 \mu \mathrm{s}), R_{\text {th }}$ and $R_{\text {in }}$ are of the same order of magnitude, but with $R_{\text {th }} \lesssim R_{\text {in }}$, we are in a transitional regime between linear and square-root-like radial growth.

In Fig. 6.5 we report the mass of gas (solid line) and the mass of vapor (dash-dotted line) inside the bubble during the bubble growth. The ratio between the two $\mathrm{m}_{\mathrm{G}} / \mathrm{m}_{\mathrm{V}}$ is of order $10^{-2}$, thus justifying the assumptions of the model. The difference between the thermal diffusivity and the mass diffusivity constants, as well as the difference between the temperature gradient and the concentration gradient driving the two diffusive phenomena, leads to different time scales and therefore to different shapes of the curves $m_{G}(t)$ and $m_{V}(t)$. We verified that the assumption of low Mach number regime holds during the whole bubble growth and collapse phase, except for the last $10 \mathrm{~ns}$ prior to collapse when the radial velocity exceeds $100 \mathrm{~m} / \mathrm{s}$, as calculated from simulations. Therefore the bubble wall velocity stays well below the critical limit of $\mathrm{Ma} \sim 0.3$ for liquid compressibility effects during the major time interval of the collapse phase.

In order to verify the robustness of predictions with respect to the initial radius chosen, we performed several calculations, varying $R_{0}$ (see Fig. 6.6). A change of this parameter by $50 \%$ in either way relative to the adopted value of $1 \mu \mathrm{m}$, inferred from the first available data point, resulted only into a small change of the growth rate of the bubble: after the first collapse it is between $+8 \%$ and $-20 \%$. The amplitude of the bubble oscillations was left unperturbed. Therefore we can conclude that the simulations are robust with respect to the choice of the initial radius.

We also explored the effects of the driving frequency on the bubble dynamics (see Fig. 6.7). At the relevant time scales, lower frequencies corresponded both to larger oscillations and larger average values of the radius. Larger oscillations at low frequencies are due to the fact that the bubble has more time to expand. Enhancement of rectified heat transfer [31-33] due to this large oscillation amplitude magnification can explain the faster growth rates at the initial stages (after $3 \mu \mathrm{s}$ ) at lower frequencies. However, the associated growth rates reverse their frequency dependence at later stages (after three microseconds). 


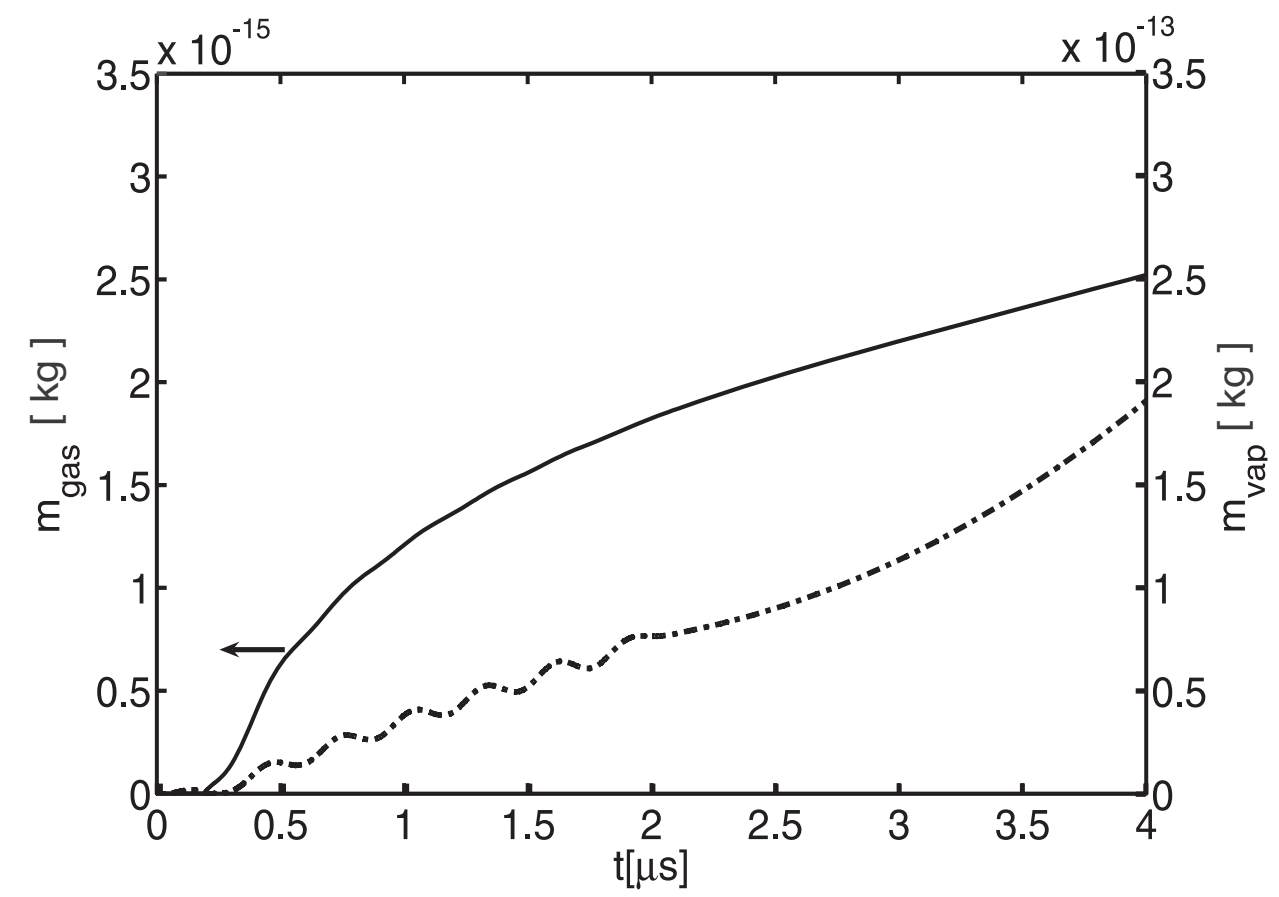

Figure 6.5: Mass of gas (solid) and vapor (dash-dot) inside a PFP bubble versus time, from simulations under the same conditions as in Fig. 6.3. Note the two order of magnitude difference between the vertical scales. 


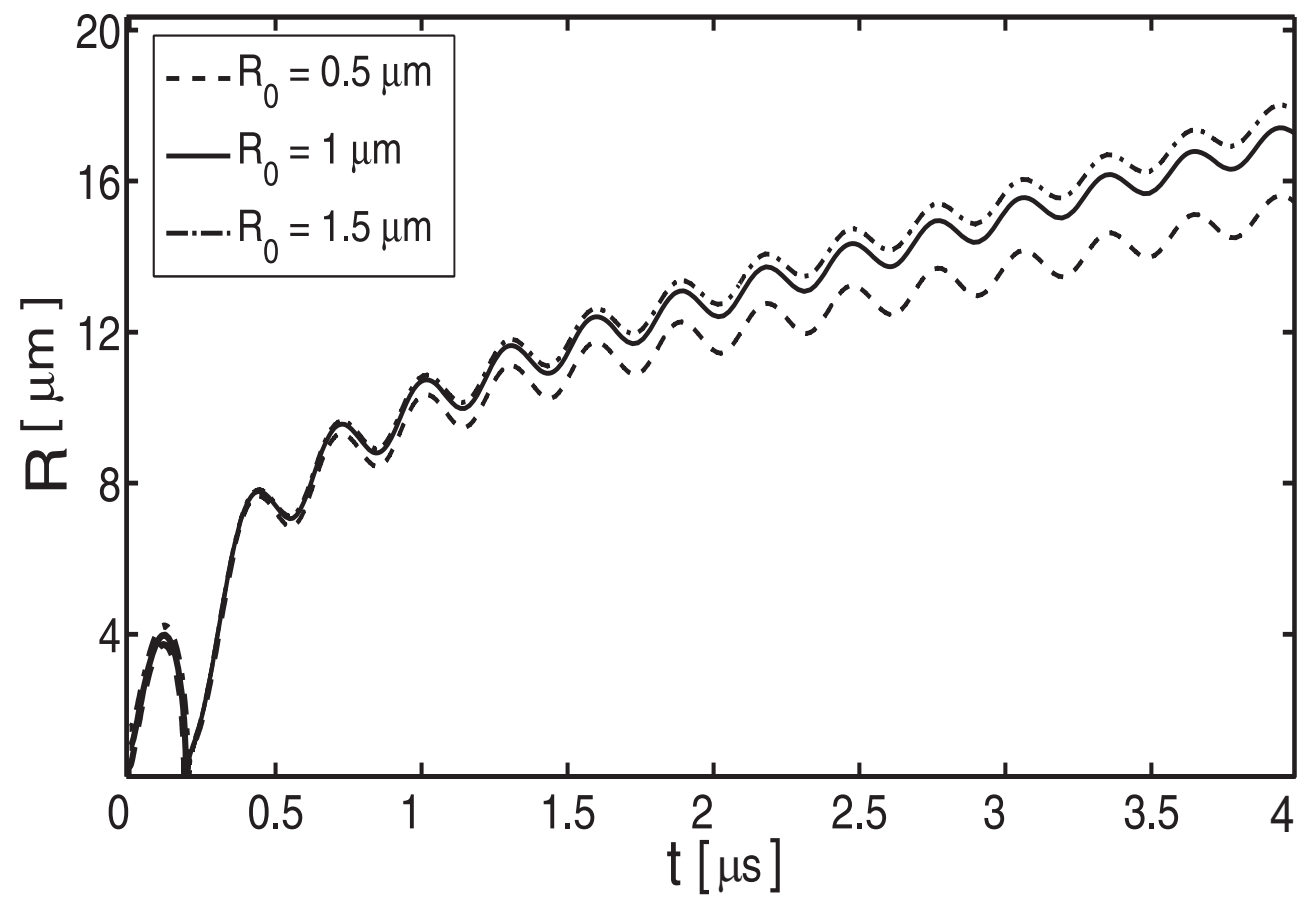

Figure 6.6: Radius-time curve $\mathrm{R}(\mathrm{t})$ from simulations for a PFP bubble for different initial radii $\mathrm{R}_{0}$, at $15^{\circ} \mathrm{C}$ superheat, driven at frequency $\mathrm{f}=3.5 \mathrm{MHz}$, pressure $\mathrm{P}_{\mathrm{a}}=$ 4.5 MPa. 


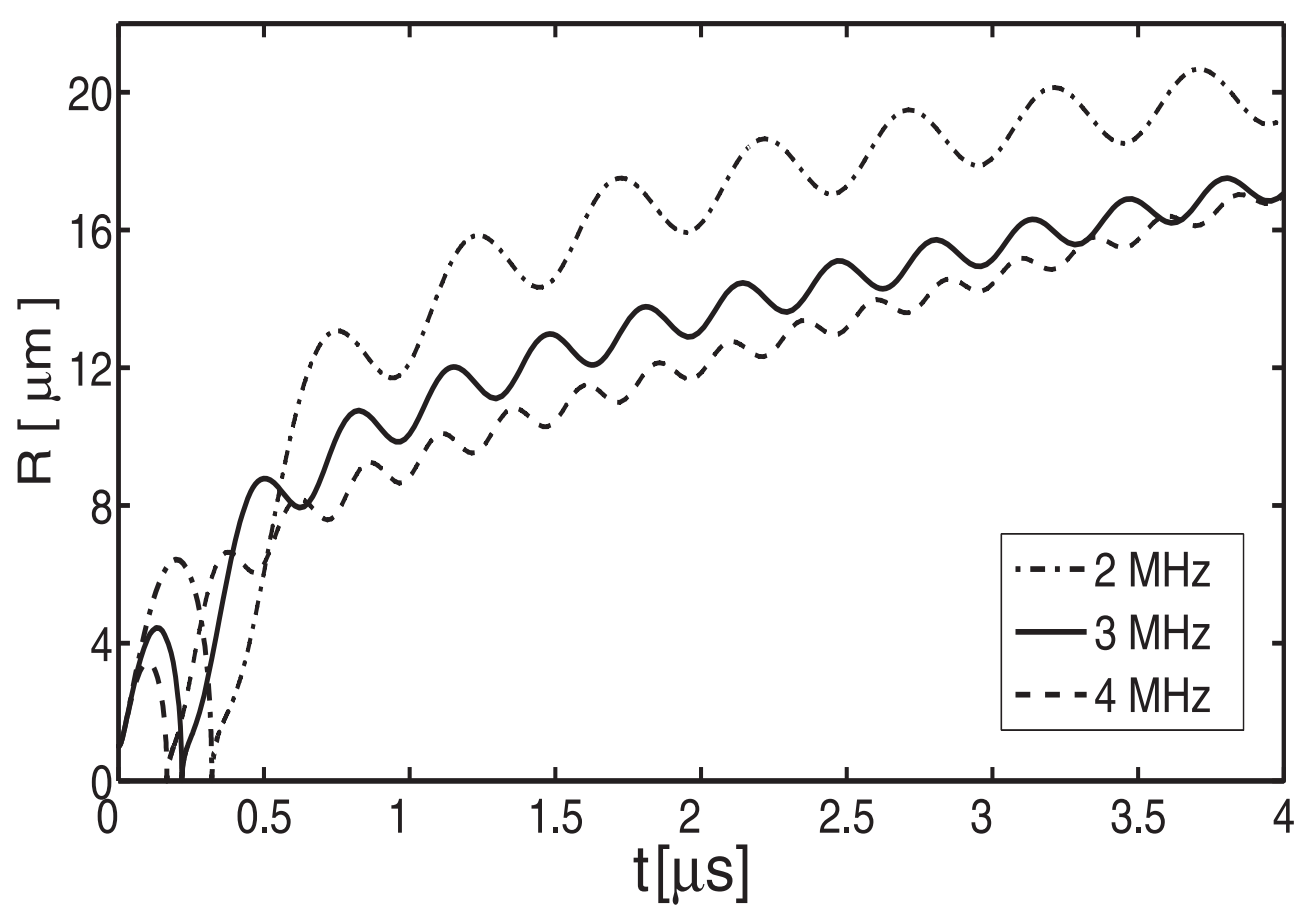

Figure 6.7: Radius-time curve $\mathrm{R}(\mathrm{t})$ from simulations for a $1 \mu \mathrm{m}$ PFP bubble driven at different frequencies with pressure $\mathrm{P}_{\mathrm{a}}=4.5 \mathrm{MPa}$, at $15^{\circ} \mathrm{C}$ superheat. 


\subsection{Summary and conclusions}

In the presented work we follow the growth dynamics of a PFP vapor bubble nucleated and driven with ultrasound, inside a PFP droplet, contained in a water medium superheated with respect to the PFP boiling point. The model we introduced here, describing the evolution of a ultrasound driven vapor bubble inside a superheated infinite medium of the same substance, succeeded to reproduce the dynamics of such a bubble within a $10 \%$ error. It is important to note that our model does not contain any free fitting parameters. It included both thermal diffusion and mass diffusion of gas (air) inside the liquid. Simulations underlined the fundamental role of gas diffusion during acoustic forcing. Though the bubble dynamics is entirely governed by evaporation and condensation processes, as the ratio between gas and vapor mass is $\mathrm{m}_{\mathrm{G}} / \mathrm{m}_{\mathrm{V}} \sim 10^{-2}$, a minimum amount of gas $(\sim 10 \%$ of the air solubility in PFP) is necessary in order to allow the bubble to survive the first collapse. When no driving is present, including or neglecting gas diffusion has no influence on the bubble growth.

Given the difficulties of experimentally monitoring the initial nucleation phase of the vapor bubble, we examined the role of the initial nucleation radius on the bubble dynamics by means of simulations. Larger initial nucleation radii lead to larger growth rates, although the oscillation amplitude is left unperturbed. We found that a change of $\pm 50 \%$ in $R_{0}$ induces a change of the bubble growth rate after the first collapse, within $30 \%$ of its original value. We also examined the effect of the driving frequency, and found that lower frequencies lead to larger oscillation amplitudes and larger bubble sizes at the relevant time scales.

\section{References}

[1] N. Y. Rapoport, Z. Gao, and A. Kennedy, "Multifunctional nanoparticles for combining ultrasonic tumor imaging and targeted chemotherapy", J. Natl. Cancer. Inst. 99, 1095-1106 (2007).

[2] P. Mohan and N. Rapoport, "Doxorubicin as a molecular nanotheranostic agent: Effect of doxorubicin encapsulation in micelles or nanoemulsions on the ultrasound-mediated intracellular delivery and nuclear trafficking.", Mol Pharmaceut. 7, 1959-1973 (2010). 
[3] O. D. Kripfgans, C. Orifici, P. Carson, K. A. Ives, O. P. Eldevik, and J. B. Fowlkes, "Acoustic droplet vaporization for temporal and spatial control of tissue occlusion: a kidney study", IEEE 52, 1101-1110 (2005).

[4] M. Zhang, M. L. Fabiilli, K. J. Haworth, F. Padilla, S. D. Swanson, O. D. Kripfgans, P. Carson, and J. Fowlkes, "Acoustic droplet vaporization for enhancement of thermal ablation by high intensity focused ultrasound", Acad Radiol. 18, 1123-1132 (2011).

[5] P. S. Sheeran, V. P. Wong, S. Luois, R. J. McFarland, W. D. Ross, S. Feingold, T. Matsunaga, and P. Dayton, "Decafluorobutane as a phase-change contrast agent for low-energy extravascular ultrasonic imaging.", UMB 37, 1518-1530 (2011).

[6] O. Couture, M. Faivre, N. Pannacci, A. Babataheri, V. Servois, P. Tabeling, and M. Tanter, "Ultrasound internal tattooing", Med. Phys 38, 1116-1123 (2011).

[7] S. Kang and C. Yeh, "Intracellular acoustic droplet vaporization in a single peritoneal macrophage for drug delivery applications", Langmuir 27, 13183-13188 (2011).

[8] N. Reznik, R. Williams, , and P. N. Burns, "Investigation of vaporized submicron perfluorocarbon droplets as an ultrasound contrast agent", UMB 37, 1271-1279 (2011).

[9] Z. Wong, O. Kripfgans, A. Qamar, J. Fowlkes, and J. L. Bull, "Bubble evolution in acoustic droplet vaporization at physiological temperature via ultra-high speed imaging", Soft Matter 7, 4009-4016 (2011).

[10] M. Fabiilli, J. Lee, O. Kripfgans, P. Carson, and J. Fowlkes, "Delivery of water-soluble drugs using acoustically triggered perfluorocarbon double emulsions", Pharm. Res. 27, 2753-2765 (2010).

[11] A. Qamar, Z. Z. Wong, J. B. Fowlkes, and J. L. Bull, "Dynamics of acoustic droplet vaporization in gas embolotherapy", Appl. Phys. Lett. 96, 143702 (2010).

[12] R. I. Nigmatulin and N. S. Khabeev, "Dynamics of vapour-gas bubbles", Izvestiya Akademii Nauk SSSR, Mekhanika Zhidkosti i Gaza 56-61 (1976). 
[13] R. I. Nigmatulin, N. S. Khabeev, and F. B. Nagiev, "Dynamics, heat and mass transfer of vapour-gas bubbles in a liquid", Int. J. Heat Mass Transfer 24, 1033-1044 (1981).

[14] M. Fanelli, A. Prosperetti, and M. Reali, "Radial oscillations of gasvapor bubbles in liquid. pt. 1. mathematical formulation", Acoustica 253-265 (1981).

[15] N. S. Khabeev, "Radial oscillations of vapor-gas bubbles", J. Appl. Mech. Tech. Phys. 6 (1987).

[16] N. S. Khabeev, "Diffusion effects in the oscillation of vapor-gas bubbles in a sound field", Int. J. Heat Mass Tran. 50, 3556-3560 (2007).

[17] G. Dassie and M. Reali, "Dynamics of an oscillating spherical gas/vapor bubble”, J. Acoust. Soc. Am. 100, 3088-3097 (1996).

[18] M. Al-Monnai and N. S. Khabeev, "Radial oscillations of soluble vaporgas bubbles in a liquid”, Fluid. Dyn. 46, 286-290 (2011).

[19] M. Fabiilli, K. Haworth, N. Fakhri, O. Kripfgans, P. Carson, and J. Fowlkes, "The role of inertial cavitation in acoustic droplet vaporization", IEEE Trans. Ultrason. Ferroelec. Freq. Contr. 56, 1006-1017 (2009).

[20] O. Shpak, T. Kokhuis, Y. Luan, D. Lohse, N. de Jong, B. Fowlkes, M. Fabiilli, and M. Versluis, "Ultrafast dynamics of the acoustic vaporization of phase-change microdroplets", J. Acoust. Soc. Am. (submitted, 2013).

[21] Y. Hao and A. Prosperetti, "The dynamics of vapor bubbles in acoustic pressure fields", Phys. Fluids 11, 2008-2019 (1999).

[22] B. Yang and A. Prosperetti, "Vapor bubble collapse in isothermal and non-isothermal liquids", J. Fluid Mech. 601, 253 - 279 (2008).

[23] L. Stricker, A. Prosperetti, and D. Lohse, "Validation of an approximate model for the thermal behavior in acoustically driven bubbles", J. Acoust. Soc. Am. 130, 3243-3251 (2011).

[24] W. Sutherland, "A dynamical theory of diffusion for non-electrolytes and the molecular mass of albumin", Phil. Mag. 9, 781-785 (1905). 
[25] J. Loschmidt, “Zur größe der luftmoleküle”, Sitzungsberichte der kaiserlichen Akademie der Wissenschaften Wien 52, 395-413 (1865).

[26] 3M, "Fluorinert liquids for electronics manufacturing, product information", http://multimedia.3m.com/mws/mediawebserver?mwsId= SSSSSufSevTsZxtUM8tZ18_9evUqevTSevTSevTSeSSSSSS- $-\& f n=$ prodinfo_fluorinert.pdf (2012).

[27] National Institute of Standards and Technology, "NIST repository", http://wtt-pro.nist.gov/wtt-pro/\#perfluoropentane (2012).

[28] L. Stricker, "Acoustic cavitation and sonochemistry", Ph.D. thesis, University of Twente (2012).

[29] T. D. Martz, P. S. Sheeran, D. Bardin, A. P. Lee, and P. A. Dayton, "Precision manufacture of phase-change perfluorocarbon droplets using microfluidics", Ultrasound Med. Biol. 37, 1952-1957 (2011).

[30] M. S. Plesset and S. A. Zwick, "The growth of vapor bubbles in superheated liquids", J. Appl. Phys. 25, 493-499 (1954).

[31] G. Trammell, "Sound waves in water containing vapor bubbles", J. Appl. Phys. 33, 1662-1670 (1962).

[32] R. Finch and E. Neppiras, "Vapor bubble dynamics", J. Acoust. Soc. Am. 53, 1402-1410 (1973).

[33] T. Wang, "Rectified heat transfer", J. Acoust. Soc. Am. 56, 1131-1143 (1974). 


\section{7 \\ Characterization of resulting bubbles ${ }^{\ddagger}$}

This work examines the acoustic characteristics of microbubbles created from vaporized submicron perfluorocarbon droplets with fluorosurfactant coating. Using ultra high-speed optical imaging, the acoustic response of individual microbubbles to low intensity diagnostic ultrasound was observed on clinically relevant timescales of hundreds of milliseconds after vaporization. It was shown that the vaporized droplets oscillate nonlinearly, and exhibit a resonant bubble size shift and increased damping relative to uncoated gas bubbles due to the presence of coating material. Unlike the commercially available lipid-coated ultrasound contrast agents, which may follow a compressiononly behaviour, vaporized droplets may exhibit expansion-dominated oscillations. It was further observed that the nonlinearity of the acoustic response of the bubbles was comparable to that of SonoVue microbubbles. These results suggest that vaporized submicron PFC droplets possess the acoustic char-

${ }_{\ddagger}^{\ddagger}$ Published as: Nikita Reznik, Guillaume Lajoinie, Oleksandr Shpak, Erik C. Gelderblom, Ross Williams, Nico de Jong, Michel Versluis, and Peter N. Burns, "On the acoustic properties of vaporized submicron perfluorocarbon droplets", Ultrasound in Medicine \& Biology 40 (6). 1379-1384 (2014).

*O. Shpak contributed to the analysis, experiments and discussions. 
acteristics necessary for their potential use as ultrasound contrast agents in clinical practice.

\subsection{Introduction}

Contrast enhanced ultrasound is a well-established diagnostic imaging technique. Microbubble ultrasound contrast agents are small, encapsulated spheres of gas, typically 1 to $5 \mu \mathrm{m}$ in diameter. Their size, similar to that of red blood cells, makes them a true blood pool agent, allowing for their use in a number of diagnostic applications, such as cancer detection [1], assessment of blood flow [2] and blood pressure measurements [3]. However, their size is also a disadvantage for some applications, as microbubbles are confined to the blood vessels, and cannot reach extravascular targets.

Submicron droplets of liquid perfluorocarbon (PFC) have been studied as a new generation of extravascular contrast agents for ultrasound. With a size of a few hundreds of nanometers in diameter, these droplets have the ability to extravasate selectively in regions of tumour growth and stay intravascular in healthy tissues, due to the enhanced permeability and retention effect [4], effectively allowing for their passive targeting to tumours. The low-boiling point droplets are acoustically inert, until exposed to a sufficiently high intensity burst of ultrasound, at which point they vaporize to produce echogenic microbubbles [5], stabilized by the coating material used to encapsulate their liquid droplet precursors for timescales of seconds to minutes [6]. Such extravascular contrast agents may be utilized for diagnostic ultrasound imaging to detect regions of tumour growth [7, 8] as well as for therapeutic applications, such as sensitizers for HIFU therapy [9] and vehicles for drug delivery [10].

For their successful application as ultrasound contrast agents (UCAs), upon vaporization the droplets should produce echogenic bubbles that respond to ultrasound in a manner similar to the currently used microbubbles on clinically relevant timescales of hundreds of milliseconds. While a number of studies have focused on the conditions necessary for droplet nucleation [5, 8, 11-14] and the vaporization process itself [15-17], only limited information is available regarding the acoustic properties of these newly created microbubbles.

In this chapter we conduct an initial study of the acoustic properties of individual vaporized PFC droplets. Using ultra high frame rate optical imaging, 
we examine the acoustic response of the fully vaporized microbubbles of different sizes to a $2.5 \mathrm{MHz}$ ultrasound pulse of low intensity (mechanical index, MI, of less than 0.1) at a few hundreds of milliseconds following the phase change. Specifically, we examine the change in bubble oscillation amplitude and non-linearity of oscillation as a function of bubble size. Furthermore, we study the influence of the presence of coating material on bubble oscillations, and compare the non-linear acoustic response of these bubbles to that of commercially available SonoVue microbubbles, in order to assess whether the vaporized droplets possess adequate acoustic properties for their potential use as UCAs.

\subsection{Materials and methods}

\subsubsection{Droplet preparations}

Droplet Preparation PFC droplet emulsions were prepared by combining water, 5\% v/v dodecafluoropentane (PFP, Fluoromed, Round Rock, TX, USA) and $0.8 \%$ v/v negatively charged fluorosurfactant Zonyl FSP (Sigma Aldrich, St. Louis, MO, USA) following a previously established protocol [15]. The samples were coarse emulsified with a vortex mixer (VM-300, Gemmy Industrial, Cherry Hill, NJ, USA) for $30 \mathrm{~s}$. Following the coarse emulsification, the solution was further emulsified with a tip-sonicator (Sonifier 250, Branson, Branson, MO, USA) for $60 \mathrm{~s}$ at $80 \%$ duty cycle. Sizing of the droplets was performed with a Zetasizer nano-sizing system (Malvern, Worcestershire, UK). This method provided droplet samples that were polydispersed in size, ranging from $100 \mathrm{~nm}$ to $1 \mu \mathrm{m}$ in diameter, with mean diameter of approximately $400 \mathrm{~nm}$.

\subsubsection{Droplet vaporization}

A schematic diagram of the experimental setup used for droplet vaporization and bubble characterization studies is shown in Fig. 8.5. Both the highintensity vaporization pulse and the low-intensity characterization pulses were sent from a $5 \mathrm{MHz}$ center frequency Olympus transducer (A308S, Olympus, Quebec City, QC, Canada), driven by an arbitrary waveform generator (8026, Tabor, Tel Hanan, Israel) amplified by an E\&I 350L (E\&I, Rochester, NY, USA) power amplifier. The transducer output was calibrated with a $0.2 \mathrm{~mm}$ 


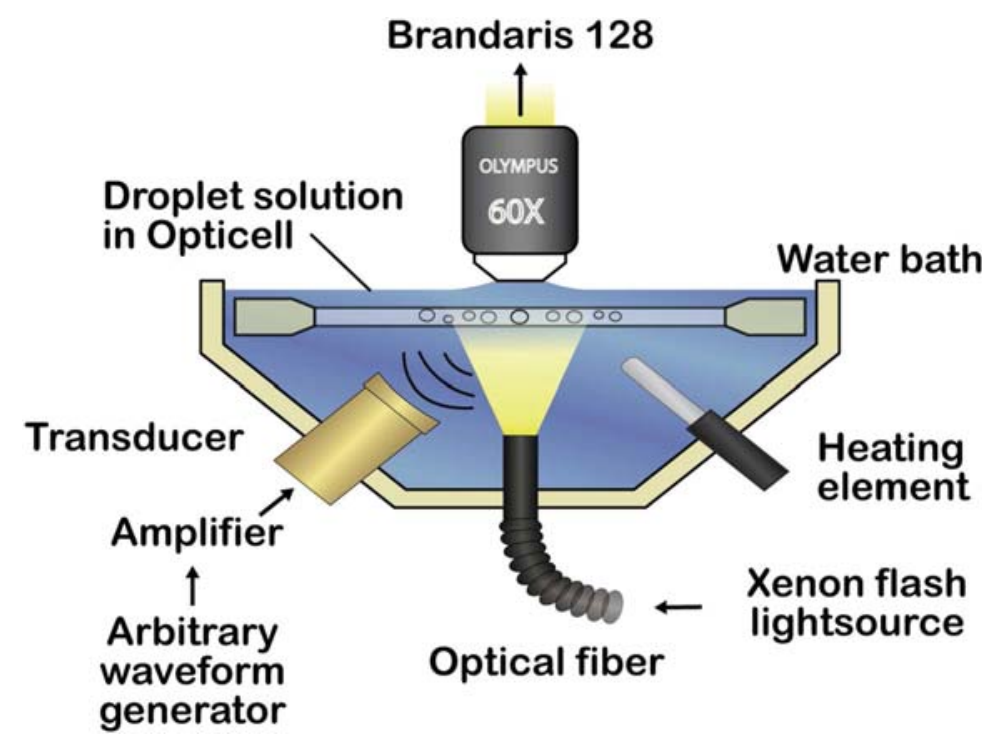

Figure 7.1: Schematic diagram of the experimental setup. Acoustic high intensity vaporization and low intensity characterization pulses were sent from the single element transducer. Detection was accomplished using a $60 \times$ objective of an Olympus microscope.

needle hydrophone probe (Precision Acoustics, Dorchester, UK). The transducer was focused on an Opticell containing the droplet sample. The droplet sample consisted of the originally prepared droplet solution, diluted 1:2000 in distilled water and degassed with a vacuum pump for a time period of at least $1 \mathrm{hr}$. The Opticell was placed under a $60 \times$ water-immersion objective $(\mathrm{NA}=1.00)$ of an Olympus BX-FM microscope, coupled to the Brandaris 128 ultra-high speed imaging system [18], providing a spatial resolution of $160 \mathrm{~nm} /$ pixel. The microscope objective was co-aligned with the transducer focus. The focal point was at $50 \mu \mathrm{m}$ above the wall of the Opticell, such that wall proximity would not have a significant effect on subsequent bubble oscillation [19]. The setup was placed in a tank filled with deionized water kept at a temperature of $37 \pm 1^{\circ} \mathrm{C}$. Droplet samples were vaporized with single ultrasound pulses, 10 cycles in length, and a peak negative pressure (PNP) of $3.5 \mathrm{MPa}$. 


\subsubsection{Vaporized droplet characterization experiment}

Following vaporization, a series of 5 low-intensity acoustic characterization pulses were sent. The pulses were 10 cycles in length, $2.5 \mathrm{MHz}$ center frequency and $116 \mathrm{kPa}$ PNP, corresponding to a mechanical index (MI) of 0.07. The pulses were sent either 80 or $100 \mathrm{~ms}$ apart. As a result, the bubbles were observed at times ranging from 80 to $500 \mathrm{~ms}$ following vaporization, based on the pulse timing interval used for the specific trial. Bubble oscillations in response to the acoustic pulses were recorded optically in a series of movies of 128 frames each, recorded at 15 million frames per second (Mfps). For our analysis, we only considered recordings that depicted bubbles in focus, such that their size and radial oscillations could be resolved with confidence. Furthermore, only images that contained either a single bubble or bubbles sufficiently far away from each other (at least $50 \mu \mathrm{s}$ apart) were considered, in order to minimize the possible coupled oscillation effects [20-22].

\subsubsection{Optical image analysis}

Bubble contours from the recorded optical images were traced using an algorithm based on the minimum cost function [23], implemented in MATLAB (MathWorks, Natick, MA, USA). The bubble radius was calculated as the mean distance from the bubble centre of mass to the edge of the bubble in a radial coordinate system. This approach provided the radius-time (R-t) curves of the bubble response to acoustic characterization pulses. In total, 30 bubble traces were analyzed.

The acoustic pressure scattered due to bubble oscillation has been calculated from the R-t curves using [24]:

$$
\left.P_{s}(r, t)=\frac{\rho}{r}\left(R(t)^{2} R \ddot{(t}\right)+2 R(t) R(t)^{2}\right),
$$

where $P_{S}$ is the scattered acoustic pressure, $\rho$ is the water density, $r$ is the distance from the bubble surface, $R$ is the bubble radius and the overdots denote the derivatives with respect to time.

The scattered pressure traces calculated from Eq. 7.1 in the time domain were Fourier-transformed, and the integrated powers in the frequency bands of $300 \mathrm{kHz}$ around the fundamental $\left(2.5 \mathrm{MHz} ; \mathrm{f}_{1}\right)$ and the second harmonic $\left(5 \mathrm{MHz} ; \mathrm{f}_{2}\right)$ were measured.

The experimental results were compared to numerical simulations for an uncoated bubble using a Rayleigh-Plesset-type equation. Furthermore, the re- 
sults were also compared to those modelled by the de Jong model for coated microbubbles [25] with shell stiffness $S_{p}=0.3 \mathrm{~N} / \mathrm{m}$ and shell friction $S_{f}=$ $3.17 \cdot 10^{-7} \mathrm{~kg} / \mathrm{s}$, identified as best fit parameters based on the oscillation amplitude results from the examined bubble traces.

\subsection{Results}

Vaporization of suspensions of submicron PFC droplets produced microbubbles ranging in size from $500 \mathrm{~nm}$ to $5 \mu \mathrm{m}$ in radius, which were stable on the clinically relevant timescales of hundreds of milliseconds. Oscillation of these newly created bubbles under low intensity diagnostic ultrasound pulses has been successfully recorded by our imaging setup. Bubble traces were examined in both the time and frequency domains, as shown in an example of a $1.7 \mu \mathrm{m}$ bubble oscillation in Fig. 7.2. The experimentally observed trace appears to fit the de Jong model well and exhibits substantially lower oscillation amplitude than that expected for an uncoated bubble (Fig. 7.2a). The power spectrum calculated from the R-t curve (Fig. 7.2b) depicts clear peaks around the fundamental frequency $(2.5 \mathrm{MHz})$ and the $2 \mathrm{nd}$ harmonic $(5 \mathrm{MHz})$, evidence of a significant nonlinear component in the bubble oscillation. None of the analyzed traces contained any observable subharmonic component.

\subsubsection{Amplitude of oscillation}

The total amplitude of bubble oscillations was measured from the maximum compression and expansion phases of the bubble, normalized to the resting radius. The amplitude as a function of bubble size is shown in Fig. 7.3. Although there is a degree of variability, the experimental results appear to correspond to the response simulated using the de Jong model. There was an experimentally observed resting bubble radius of $R_{\text {res }}=1.75 \mu \mathrm{m}$, for which the bubble oscillation amplitude was at maximum. This resting radius is in concordance with that simulated by the de Jong model and significantly different from the one of an uncoated bubble of $R_{0}=1.3 \mu \mathrm{m}$. The measured amplitude of bubble oscillation is also in agreement with the results simulated by the de Jong model, and is substantially lower than that exhibited by the simulated uncoated bubble response.

Furthermore, it was observed that the bubbles did not always oscillate symmetrically. The smaller bubbles, with radii below Rres, preferred expan- 

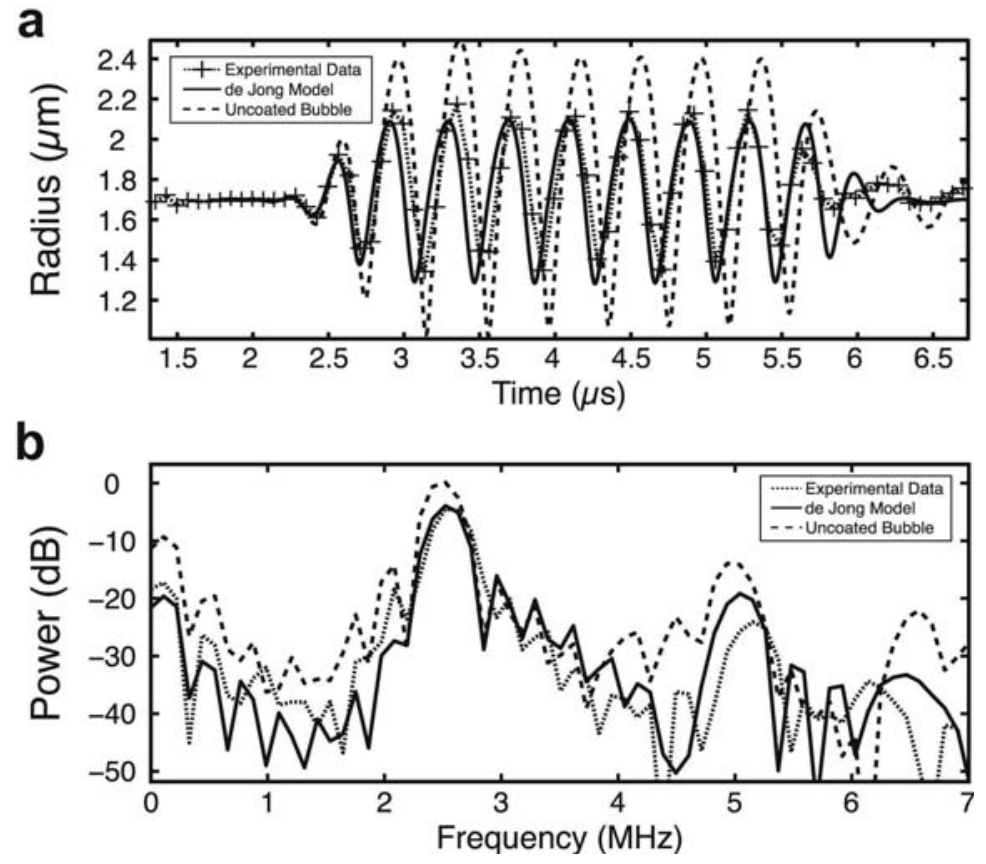

Figure 7.2: (a) Radius-time trace of a bubble with initial radius of $\mathrm{R}_{0}=1.7 \mu \mathrm{m}$, with a fit by the de Jong model and the trace simulated for an uncoated bubble for the same size. (b) Frequency spectrum of the bubble oscillation, as calculated from the observed radius-time curve, together with the de Jong model fit and the spectrum obtained from simulated uncoated bubble oscillation. 


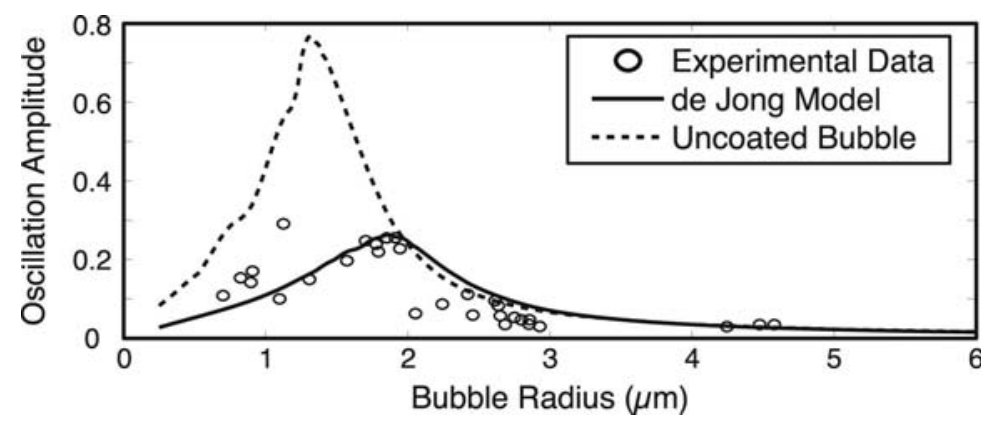

Figure 7.3: Amplitude of bubble oscillation normalized to the bubbles' resting radii, as a function of the bubbles' resting radii. Shown are the experimental data, the best fit of the de Jong model and the simulated response from an uncoated bubble. A decrease in amplitude of oscillation and increase in resonant bubble size for the experimental results compared with the uncoated bubble simulation is apparent.

sion dominated oscillations. At the same time, bubbles bigger than approximately $2 \mu \mathrm{m}$ in radius exhibited largely symmetric oscillations.

\subsubsection{Nonlinearity of emitted pressures}

In order to assess the non-linearity in the emitted acoustic signal from the bubbles, pressure-time curves were calculated from the experimentally observed and the simulated R-t plots, and analyzed in the frequency domain. In order to examine the degree of non-linearity, the integrated powers in the fundamental and 2nd harmonic bands were calculated separately as a function of bubble size, as shown in Fig. 7.4. The de Jong model appears to describe the bubble oscillation in the fundamental band and the general magnitude of the power of the 2 nd harmonic band. The ratio of the power in the 2 nd harmonic band to the power in the fundamental band was on the order of -5 to $-15 \mathrm{~dB}$, depending on bubble size.

\subsection{Discussion}

\subsubsection{Bubble acoustic behaviour}

In this work we look at the acoustic response of microbubbles created from their liquid droplet precursors at times ranging from 80 to $500 \mathrm{~ms}$ following 

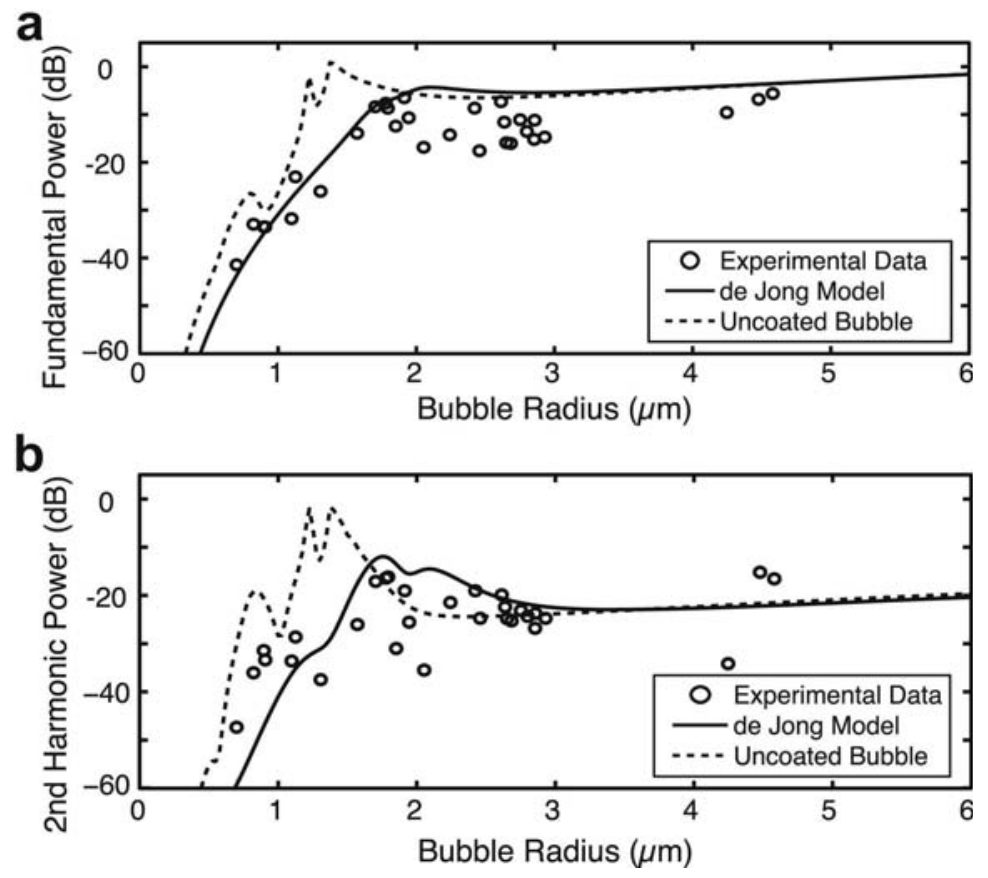

Figure 7.4: Measured power of scattered acoustic pressure in the (a) fundamental and (b) second harmonic bands for experimentally obtained data and the simulated model results. The ratio of power in the second harmonic band to that in the fundamental band ranges from 5 to $15 \mathrm{~dB}$. 
vaporization. It is envisioned that the timescales of hundreds of milliseconds are the most relevant clinically as they are on the same order as the inter-frame times of typical clinical US scanners (operating at tens of $\mathrm{Hz}$ frame rates). The MI of the ultrasound characterization pulse used here $(\mathrm{MI}=0.07)$ is lower than the typical MI of diagnostic ultrasound used in the clinic. However, the lower ultrasound pressure settings minimized the possibility of bubble cavitation or gas loss and other deflation effects, while allowing for the study of associated non-linear oscillation behaviour of the bubbles.

Although the observed acoustic response of the bubbles has a significant degree of variability, general trends which can help assess the acoustic response of the vaporized PFC droplets for their potential application as UCAs are visible. For the characterization pulse used (2.5 MHz, $116 \mathrm{kPa} P N P)$, there is a bubble radius of $R_{\text {res }}=1.75 \mu \mathrm{m}$ associated with maximum bubble oscillation amplitude (Fig. 7.3). For simplicity, we refer to $R_{\text {res }}$ as the resonant size. However, it is important to note that at the pressures investigated here, the bubbles exhibit a non-linear response, and the size of maximum oscillation $R_{\text {res }}$ in this case may be significantly different from the resonant size of the bubble in the linear regime [26]. The bubble resonant size is larger than that expected for an uncoated bubble, while the amplitude of bubble oscillation is lower than that of an uncoated bubble. A shift in $R_{\text {res }}$ and decrease in oscillation amplitude are characteristic of the presence of a viscoelastic shell material. It has been shown previously [6] that following vaporization, the newly created bubbles retain the original shell material stabilizing the droplet precursors. The results of the present study suggest that the surfactant coating originally on the droplets affects the acoustic behaviour of the newly created microbubbles.

Simulated bubble response using the de Jong model describes the experimentally observed trends. The fitted shell elasticity parameter of $S_{p}=$ $0.3 \mathrm{~N} / \mathrm{m}$ is substantially lower than the measured value for clinically used SonoVue microbubbles of $S_{p}=1.1 \mathrm{~N} / \mathrm{m}$, however, the fitted viscosity parameter of $S_{f}=3.17 \cdot 10^{-7} \mathrm{~kg} / \mathrm{s}$ appears to be similar to that of SonoVue [27].

For the ultrasound pulses used here, vaporized PFC droplets do not appear to exhibit the characteristic compression-only behaviour observed in some cases for lipid coated microbubbles [24]. On the contrary, bubbles smaller than the resonant size exhibit an expansion-dominated behaviour, indicative of higher resistance to compression than expansion. 


\subsubsection{Suitability of vaporized PFC droplets for the role of UCAs}

In order to assess the applicability of vaporized PFC droplets as UCAs, it is instructive to compare the observed bubble response to the previously studied acoustic responses from clinically used microbubbles, such as SonoVue. It has been shown that smaller SonoVue microbubbles favour compressiondominated oscillations or even compression-only behaviour due to the buckling of the lipid bubble shells $[28,29]$. This comes in sharp contrast with the observed behaviour of bubbles under consideration in this study that show a preference for expansion-dominated behaviour. The lack of compressiondominated oscillations and no detectable subharmonic signal suggests that, unlike the commercially available lipid-coated UCAs, vaporized fluorosurfactant coated droplets under study do not exhibit shell buckling behaviour, even at the relatively high oscillation amplitudes of up to $25 \%$ of the bubble radius.

At the same time, for the purposes of their application as UCAs, the nonlinearity of the scattered pressure due to bubble oscillation is of paramount importance, as nonlinear scattering serves as the basis for contrast-specific US imaging techniques, such as pulse-inversion imaging [30]. From the experimentally observed data, the ratio of scattered acoustic signal powers in the $2 \mathrm{nd}$ harmonic band to fundamental band is on the order of -5 to $-15 \mathrm{~dB}$ (Fig. 7.4) for US at a frequency of $2.5 \mathrm{MHz}$ and $\mathrm{MI}$ of 0.07 . These values are similar to the value of approximately $-12 \mathrm{~dB}$ observed for SonoVue microbubbles populations [31] at the same MI for a $2.25 \mathrm{MHz}$ excitation frequency. It should be noted, however, that the results of this study for vaporized droplets were obtained by optical observation of single bubbles, while the characterization results for SonoVue in the referenced works [27, 31] were obtained by an acoustic interrogation of bubble populations. Thus, only a qualitative comparison can be done. While there is similarity in the acoustic response of the two types of bubbles, more detailed and controlled examinations are necessary to quantitatively compare the two. The results presented here show that, on clinically relevant timescales, the acoustic response of vaporized droplets is similar to that of clinically used microbubble agents and supports their future utilization as UCAs. 


\subsection{Conclusion}

This work investigated the acoustic characteristics of vaporized submicron droplets on clinically relevant timescales. It was shown that following vaporization with ultrasound, the newly created microbubbles oscillate nonlinearly and exhibit coating material-induced effects on oscillation that can be described by the de Jong coated bubble dynamics model. The coating material on the surface of the bubbles induces a shift in the resonance frequency and a substantial amount of damping due to shell viscosity.

Unlike lipid-coated UCAs, the fluorosurfactant coated bubbles used in this study do not exhibit buckling behaviour, and tend to feature expansiondominated oscillations for smaller bubbles. At the same time, it was shown that vaporized PFC droplets scatter ultrasound in a manner similar to the currently clinically used microbubble contrast agents. These results suggest that stable vaporized PFC droplets possess the adequate acoustic characteristics to support their potential use as UCAs in the clinic.

\section{References}

[1] P. Burns and S. Wilson, "Focal liver masses: Enhancement patterns on contrast-enhanced imagesconcordance of us scans with ct scans and $\mathrm{mr}$ images", Radiology 242, 162-174 (2007).

[2] J. M. Hudson, R. Williams, B. Lloyd, M. Atri, T. K. Kim, G. A. Bjarnason, and P. N. Burns, "Improved flow measurement using microbubble contrast agents and disruption-replenishment: clinical application to tumour monitoring", Ultrasound Med. Biol. 37, 1210-1221 (2011).

[3] C. Tremblay-Darveau, R. Williams, and P. Burns, "Measuring absolute blood pressure using microbubbles", Ultrasound Med. Biol. 40, 775787 (2014).

[4] H. Maeda, J. Wu, T. Sawa, Y. Matsumura, and K. Hori, "Tumor vascular permeability and the epr effect in macromolecular therapeutics: A review", J. Control Release 165, 271-284 (2000).

[5] Reznik, R. Williams, and P. Burns, "Investigation of vaporized submicron perfluorocarbon droplets as an ultrasound contrast agent", Ultrasound Med. Biol. 37, 1271-1279 (2011). 
[6] N. Reznik, M. Seo, R. Williams, E. Bolewska-Pedyczak, M. Lee, N. Matsuura, J. Gariepy, F. Foster, and P. Burns, "Optical studies of vaporization and stability of fluorescently labelled perfluorocarbon droplets", Phys. Med. Biol. 57, 7205-7217 (2012).

[7] T. Matsunaga, P. Sheeran, S. Luois, J. Streeter, L. Mullin, B. Banerjee, and P. Dayton, "Phase-change nanoparticles using highly volatile per-fluorocarbons: Toward a platform for extravascular ultrasound imaging", Theranostics 2, 1185-1198 (2012).

[8] R. Williams, C. Wright, E. Cherin, N. Reznik, M. Lee, I. Gorelikov, F. Foster, N. Matsuura, and P. Burns, "Characterization of submicron phase-change perfluorocarbon droplets for extravascular ultrasound imaging of cancer", Ultrasound Med. Biol. 39, 475-489 (2013).

[9] L. Phillips, C. Puett, P. Sheeran, P. Dayton, G. Miller, and T. Matsunaga, "Phase-shift perfluorocarbon agents enhance high intensity focused ultrasound thermal delivery with reduced near-field heating", J. Acoust. Soc. Am. 134, 1473-1482 (2013).

[10] N. Rapoport, "Phase-shift, stimuli-responsive perfluorocarbon nanodroplets for drug delivery to cancer", Wiley Interdiscip. Rev. Nanomed. Nanobiotechnol. 4, 492-510 (2012).

[11] O. Couture, A. Urban, A. Bretagne, L. Martinez, M. Tanter, and P. Tabeling, "In vivo targeted delivery of large payloads with an ultrasound clinical scanner", Med. Phys. 39, 5229-5237 (2012).

[12] O. Kripfgans, J. Fowlkes, D. Miller, O. Eldevik, and P. Carson, “Acoustic droplet vaporization for therapeutic and diagnostic applications", Ultrasound Med. Biol. 114, 1177-1189 (2000).

[13] P. Sheeran, V. Wong, S. Luois, R. McFarland, W. Ross, S. Feingold, T. Matsunaga, and P. Dayton, "Decafluorobutane as a phase-change contrast agent for low-energy extravascular ultrasonic imaging", Ultrasound Med. Biol. 37, 1518-1530 (2011).

[14] O. Shpak, L. Stricker, M. Versluis, and D. Lohse, "The role of gas in ultrasonically driven vapor bubble growth", Phys. Med. Biol. 58, 25232535 (2013). 
[15] N. Reznik, O. Shpak, E. Gelderblom, R. Williams, N. de Jong, M. Versluis, and P. Burns, "The efficiency and stability of bubble formation by acoustic vaporization of submicron perfluorocarbon droplets", Ultrasonics 53, 1368-1376 (2013).

[16] P. Sheeran, T. Matsunaga, and P. Dayton, "Phase-transition thresholds and vaporization phenomena for ultrasound phase-change nanoemulsions assessed via high-speed optical microscopy”, Phys. Med. Biol. 58, 4513-4534 (2013).

[17] O. Shpak, T. Kokhuis, Y. Luan, D. Lohse, N. de Jong, J. Fowlkes, M. Fabiilli, and M. Versluis, "Ultrafast dynamics of the acoustic vaporization of phase-change microdroplets", J. Acoust. Soc. Am. 134, 1610-1621 (2013).

[18] C. Chin, C. Lance, J. Borsboom, F. Mastik, M. Frijlink, N. de Jong, M. Versluis, and D. Lohse, "Brandaris 128: A digital 25 million frames per second camera with 128 highly sensitive frames", Rev. Sci. Instrum. 74, 5026-5034 (2003).

[19] V. Garbin, D. Cojoc, E. Ferrari, E. D. Fabrizio, M. Overvelde, S. van der Meer, N. de Jong, D. Lohse, and M. Versluis, "Changes in microbubble dynamics near a boundary revealed by combined optical micromanipulation and high-speed imaging", Appl. Phys. Lett. 90, 114-103 (2007).

[20] J. Allen, D. Kruse, P. Dayton, and K. Ferrara, "Effect of coupled oscillations on microbubble behavior", J. Acoust. Soc. Am. 114, 1678-1690 (2003).

[21] V. Garbin, B. Dollet, M. Overvelde, D. Cojoc, E. D. Fabrizio, L. van Wijngaarden, A. Prosperetti, N. de Jong, D. Lohse, , and M. Versluis, "History force on coated microbubbles propelled by ultrasound", Phys. Fluids. 21, 092003 (2009).

[22] T. Kokhuis, V. Garbin, K. Kooiman, B. Naaijkens, L. Juffermans, O. Kamp, A. van der Steen, M. Versluis, and N. de Jong, "Secondary bjerknes forces deform targeted microbubbles", Ultrasound Med. Biol. 39, 490-506 (2013).

[23] S. V. der Meer, B. Dollet, M. Voormolen, C. Chin, A. Bouakaz, N. de Jong, M. Versluis, and D. Lohse, "Microbubble spectroscopy of ultrasound contrast agents", J. Acoust. Soc. Am. 39, 648-656 (2007). 
[24] J. Sijl, M. Overvelde, B. Dollet, V. Garbin, N. de Jong, D. Lohse, and M. Versluis, "compression-only behavior: A second-order nonlinear response of ultrasound contrast agent microbubbles", J. Acoust. Soc. Am. 129, 1729-1739 (2011).

[25] N. D. Jong, R. Cornet, and C. Lancee, "Higher harmonics of vibrating gas-filled microspheres: 1”, Ultrasonics 32, 1678-1690 (1994).

[26] M. Overvelde, V. Garbin, J. Sijl, B. Dollet, N. de Jong, D. Lohse, and M. Versluis, "Nonlinear shell behavior of phospholipid-coated microbubbles”, Ultrasound Med. Biol. 36, 2080-2092 (2010).

[27] J. Gorce, M. Arditi, and M. Schneider, "Influence of bubble size distribution on the echogenicity of ultrasound contrast agents: A study of sonovue", Invest. Radiol. 35, 661-671 (2000).

[28] N. D. Jong, M. Emmer, C. Chin, A. Bouakaz, F. Mastik, D. Lohse, and M. Versluis, "compression-only behavior of phospholipid-coated contrast bubbles", Ultrasound Med. Biol. 33, 653-656 (2007).

[29] M. Emmer, A. van Wamel, D. Goertz, and N. de Jong, "The onset of microbubble vibration", Ultrasound Med. Biol. 33, 941-949 (2007).

[30] D. Simpson, C. Chin, and P. Burns, "Pulse inversion doppler: A new method for detecting nonlinear echoes from microbubble contrast agents", IEEE Trans. Ultrason. Ferroelec. Freq. Contr. 130, 372-382 (1999).

[31] M. Schneider, "Characteristics of sonovue", Echocardiogr-J Card 16, 743-746 (1999). 


\section{8 Monodisperse droplets for ADV}

Droplet vaporization dynamics as well as its nucleation strongly depends on the droplet size. Thus, to understand the other driving parameters it is important to fabricate monodisperse droplets in order to have a high degree of repeatability and control over the vaporization process. The aim of this chapter is to study the production of monosized PFC droplets and to investigate the nucleation and growth of monodisperse droplets at a nanoseconds time scale. We fabricate glass chips with a step geometry, which allow us to produce monodisperse submicron droplets. We show that the vaporization of monodisperse droplets have high degree of symmetry and that the activation threshold variations are as small as $70 \mathrm{kPa}$ at $3.5 \mathrm{MHz}$ frequency.

\subsection{Introduction}

Liquid emulsion droplets of a radius of $0.2-2 \mu \mathrm{m}$ composed of perfluoropentane (PFP) and a drug (Doxorubicin) are currently being studied as a potential system for tumor imaging, embolotherapy and for local drug delivery [1]. The

\footnotetext{
$\ddagger$ To be submitted as: Oleksandr Shpak, Tim Segers, Lingling Shui, Guillaume Lajoinie, Jan Eijkel, Nico de Jong, Detlef Lohse, and Michel Versluis, "Monodisperse droplets for ADV'.
} 
droplets have the ability to extravasate through hyperpermeable tumor blood vessel walls, and to accumulate in interstitial tissue. PFP has a boiling temperature of $29^{\circ} \mathrm{C}$, however surrounded by medium of up to $50^{\circ} \mathrm{C}$ droplets stay in the liquid phase until they are triggered and nucleated using focused ultrasound. The nucleation event converts the droplets into gas bubbles, while the contained drugs are released during the subsequent explosive vaporization of the droplet.

In a previous study [1-3] it was shown that droplets, prior to vaporization, undergo deformation and oscillatory motion driven by the compressibility of the surrounding fluid at the ultrasound frequency with an amplitude of 200$400 \mathrm{~nm}$. The growth of the vapor nucleus was observed to have speed up to $40 \mathrm{~m} / \mathrm{s}$ [4]. The vapor bubble growth on later stages was demonstrated to be limited by heat transfer $[4,5]$. Nucleation sites inside the droplets experience vaporization/condensation cycles in phase with the applied ultrasound. However the initiation of the vaporization has a strong dependence on the droplet size [6]. The resulting vapor bubble sizes after complete vaporization also strongly depends on the initial droplet size [7]. For both therapy [8] and imaging [9] purposes it is important to employ monodisperse droplets which all have the same properties with the same nucleation threshold and the same excitation bubble resonance frequency which governed by the resulting bubble size.

The aim of this study is to produce monosized PFP droplets and to record the nucleation and growth at a nanoseconds time scale. We demonstrate that with proposed nano-microchannel interface chip structure droplets can be produced probably as small as $260 \mathrm{~nm}$ in radius, which are one of the smaller monodisperse PFP droplets reported to date [10-14].

Previous studies have reported the activation of perfluorocarbon droplets below their boiling point and the consecutive release of a drug [8]. In this study we also aim to visualize how perfluoropentane droplets below boiling point react to the exposure to focused ultrasound.

\subsection{Materials and methods}

\subsubsection{Nano-microchannel chip fabrication}

The microfluidic chips were made of $500 \mu \mathrm{m}$ thick Borofloat glass (Schott Technical Glasses, Germany). The fabrication process is shown in Fig. 8.1 


\section{Borofloat Glass}

(1)

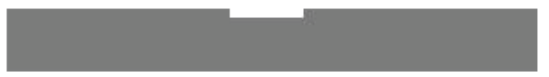

(2)

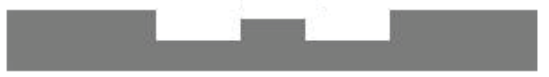

(3)

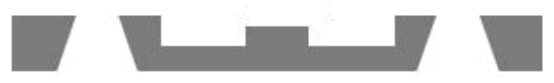

(4)

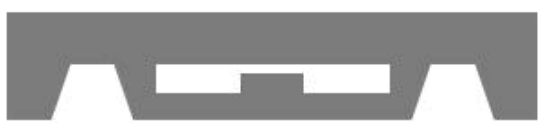

Figure 8.1: Schematic of the microfluidic chip fabrication process. For more details [15].

1) Borofloat wafer was first cleaned, photolithographed and wet-etched by a BHF mixture solution to create nanochannels of $80 \mathrm{~nm}, 180 \mathrm{~nm}, 290 \mathrm{~nm}$, $390 \mathrm{~nm}$ and $1150 \mathrm{~nm}$ in height.

2) A thin layer gold/chromium $(\mathrm{Cr} / \mathrm{Au})$ was sputtered onto the nano-patterned substrate with a thickness of $15 / 150 \mathrm{~nm}$ (Cr is used as an adhesive layer for $\mathrm{Au}$ onto the glass substrate); then the $\mathrm{Au} / \mathrm{Cr}$ layer was patterned by photolithography and wet-etched in $\mathrm{Au}$ and $\mathrm{Cr}$ etchants (Merck); the patterned glass substrate was then wet-etched in $25 \% \mathrm{HF}$ solution to create microchannels prior to $\mathrm{Cr} / \mathrm{Au}$ removal.

3) Access holes were made from the backside of the glass wafer by powderblasting

4) The patterned wafer was thermally bonded to a bare glass wafer at $650^{\circ} \mathrm{C}$.

The thermally bonded glass wafers were diced to obtain individual glass chips of $10 \mathrm{~mm} \times 20 \mathrm{~mm}$ in size. Chips were fixed in a custom-built chip holder and connected to syringes via capillary tubing (OD 1/16 inch, ID $100 \mu \mathrm{m}$ ) and Nanoport connectors including a filter (INACOM INSTRUMENTS BV, Upchurch Scientific, the Netherlands). 


\subsubsection{PFP droplet production}

The formation of a liquid filament is in most cases a prerequisite step for producing a droplet. In a certain range of physical parameters and geometrical configurations, the liquid filament size, and therefore the droplet size, is determined by the nanochannel geometry. It was found in earlier work that it is mainly its height that determines the Laplace pressure [15]. PFC droplet emulsions up to $3.8 \mu \mathrm{m}$ radius were produced using different sizes of nanochannels (Fig. 8.3) . The microchannel, see Fig. 8.2 supplied the 99\% $\mathrm{v} / \mathrm{v}$ water and $1 \%$ v/v negatively charged fluorosurfactant Zonyl FSP (Sigma Aldrich, St. Louis, MO, USA) flow. The nanochannel supplied the dodecafluoropentane (PFP, Fluoromed, Round Rock, TX, USA) flow. The nanochannel to microchannel flow rate ratio was approximately as 1:20. In order to prevent the perfluoropentane from vaporization due to the temperature rise from the fiber illumination the ambient temperature was set to $16^{\circ} \mathrm{C}$. The droplets collected by the $1150 \mathrm{~nm}$ height chip were sized using optical images recorded by the Brandaris camera [16]. Their mean radius was determined to be $3.8 \mu \mathrm{m}$ with 17\% FWHM size distribution. The optical resolution of the images of the droplets collected with the $80 \mathrm{~nm}, 180 \mathrm{~nm}, 290 \mathrm{~nm}$ and $390 \mathrm{~nm}$ nanochannels was not sufficient to determine the droplet size accurately. The ratio $\frac{R_{d}}{h}=3.3$, obtained for the $R_{d}=3.8 \mu \mathrm{m}$ radius droplets, where $R_{d}$ is the droplet radius and $h$ is the height of nanochannel, was linearly extrapolated to determine the the droplet sizes collected with the $80 \mathrm{~nm}, 180 \mathrm{~nm}, 290 \mathrm{~nm}$ and $390 \mathrm{~nm}$ nanochannels following the scaling arguments given in [15]. The radius of the droplets collected with the smallest $(h=80 \mathrm{~nm})$ nanochannel was therefore estimated to be $R_{d}=260 \mathrm{~nm}$.

The droplets of $7.4 \mu \mathrm{m}$ and $13 \mu \mathrm{m}$ radius were produced by the methods desribed by [8] and [9] respectively, and sized using optical images recorded by the Brandaris camera [16]. The FWHM of their size distribution was $17 \%$ and $14 \%$ respectively.

The flow rate is controlled by a high-precision syringe pump (Harvard Apparatus, PHD 2000, Holliston, MA, USA). Both water and perfluorocarbon flows were filtered by an in-line syringe filter to prevent channel clogging by dust particles.

The production of the droplets was imaged using a high-speed camera (Photron SA1.1) connected to a microscope (Olympus BX-FM modular system) equipped with a water-immersed objective (Olympus, LUMPlanFL). The imaging resolution was $0.5 \mu \mathrm{m}$ per pixel. The system was illuminated in 


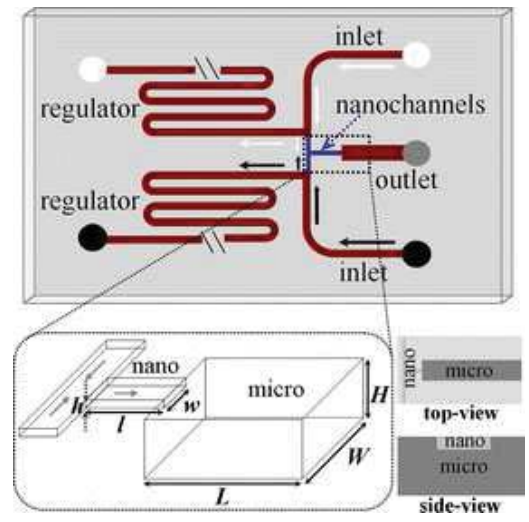

Figure 8.2: Sketch of the nano-micro fluidic chip design. When two immiscible liquids (oil inner liquid and oil outer liquid) step from the nanochannel to the microchannel monodisperse droplets form at the nano-microchannel interface.

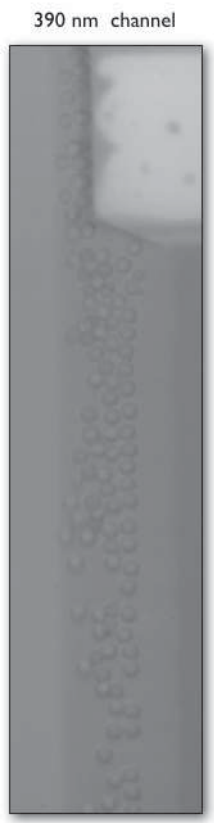

$1.3 \mu \mathrm{m}$ radius

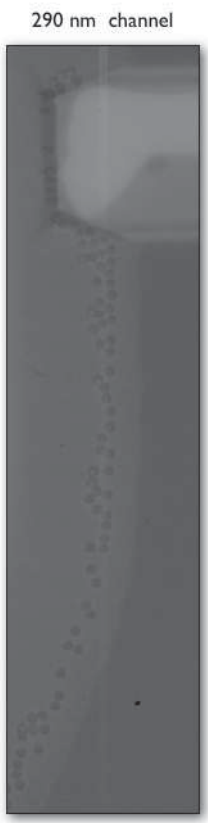

$0.94 \mu \mathrm{m}$ radius

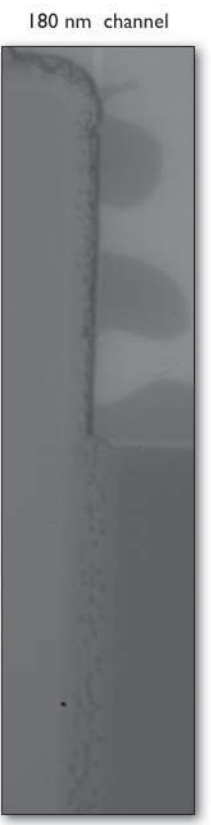

$0.59 \mu \mathrm{m}$ radius

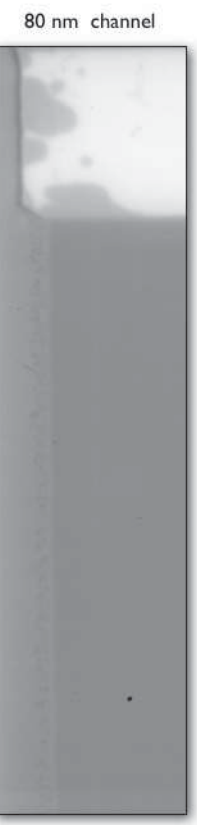

$0.26 \mu \mathrm{m}$ radius

Figure 8.3: The droplet production process with $390 \mathrm{~nm}, 290 \mathrm{~nm}, 180 \mathrm{~nm}$ and $80 \mathrm{~nm}$ nanochannel heights. 


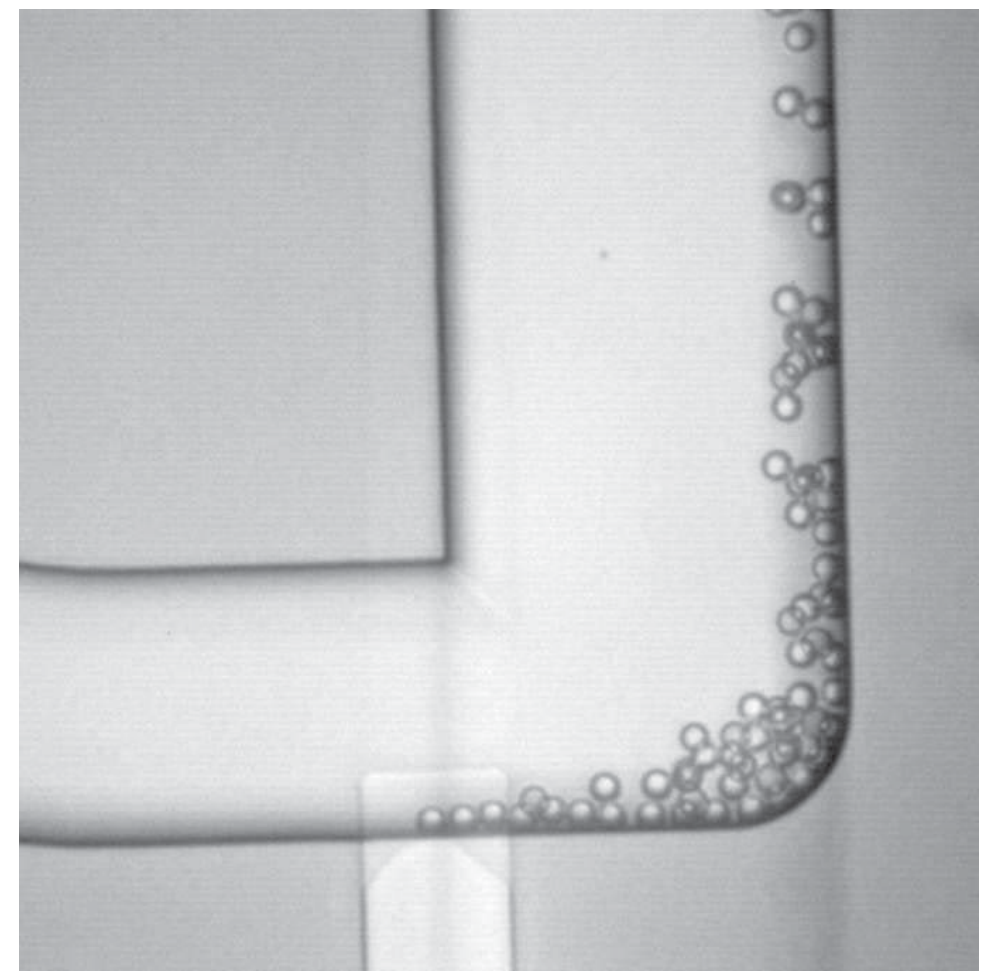

Figure 8.4: Snapshots of the formation process of droplets with a radius of $3.8 \mu \mathrm{m}$ by the $1150 \mathrm{~nm}$ height nanochannel

transmitted light mode using fiber illumination (Olympus ILP-1) connected to a collimation objective $(10 \times$ Olympus Plan Achromat NA $=0.25)$ positioned below the microfluidic chip to maximize the light intensity at the imaging position. All high-speed recordings were captured at 3000 frames per second (333 $\mu$ s interframe time) and the exposure time was set to $16 \mu$ s to minimize motion blur (Fig. 8.4). The generated droplets were found to be stable for at least three weeks.

\subsubsection{Droplet vaporization}

A schematic diagram of the experimental setup used for the droplet vaporization and the bubble characterization studies is shown in Fig. 8.5. The ultrasound pulses were sent from 3.5 MHz and 5.0 MHz center frequency transducers (A308S, Olympus, Antwerpen, Belgium), driven by an arbitrary 
waveform generator (8026, Tabor, Tel Hanan, Israel) amplified by an ENI 2000L (ENI, Rochester, NY, USA) power amplifier. The transducer output was calibrated with a $0.2 \mathrm{~mm}$ needle hydrophone probe (Precision Acoustics, Dorchester, UK). The ultrasound was focused in an Opticell containing the monodisperse droplet sample. The droplet sample consisted of the originally prepared droplet solution diluted to 1:5000 in distilled water. The Opticell was placed under a $40 \times$ water-immersion objective $(\mathrm{NA}=1.00)$ of an Olympus BX-FM microscope, coupled to the Brandaris 128 ultra-high speed imaging system [16], providing a spatial resolution of $163 \mathrm{~nm}$ per pixel. The microscope objective was co-aligned with the transducer focus. The setup was placed in a tank filled with deionized water, with temperature control between a $11.8-41.8^{\circ} \mathrm{C}$ interval. The droplet samples were vaporized with a single ultrasound pulse composed of 4-10 cycles in length, and a peak negative pressure (PNP) of 3.0, 3.7 and 4.6 MPa. The measurement protocol was as follows: the activation of monodisperse perfluorocarbon droplets was imaged at a nanoseconds time scale by ultra-high speed imaging facility. For each individual ambient temperature we repeat the experiment 3-5 times in order to verify the repeatability of the process.

\subsection{Results and discussion}

\subsubsection{Nucleation and scattering}

Monodisperse droplets have nucleation spots at the same position as was previously shown in Chapter 4. However, clusters of droplets can have different nucleation patterns than the single ones. This can be explained by the strong scattering of the ultrasound wave by the droplet spheres. Not only the position of nuclei initiation can change, but also their activation thresholds. It was observed that clusters of droplets are much easier to vaporize, than the single ones.

Collections of monodisperse droplets arranged into symmetric order can for the ultrasound wave serve as an analogy to a crystal lattice structure for Bragg diffraction in solid state physics. Moreover, clusters of monodisperse droplets can form symmetric nucleation patterns. For example Fig. 8.6 shows a snapshot at $t=0 \mu$ s of the arrangement of monodisperse droplets with a radius of $13.7 \mu \mathrm{m}$. The snapshot at $t=0.4 \mu$ s captures the moment of the first nucleation. The nucleus appears at the center of the droplet sitting in the middle of the droplet cluster. At $t=0.9 \mu$ s, after the vapor bubble of the 


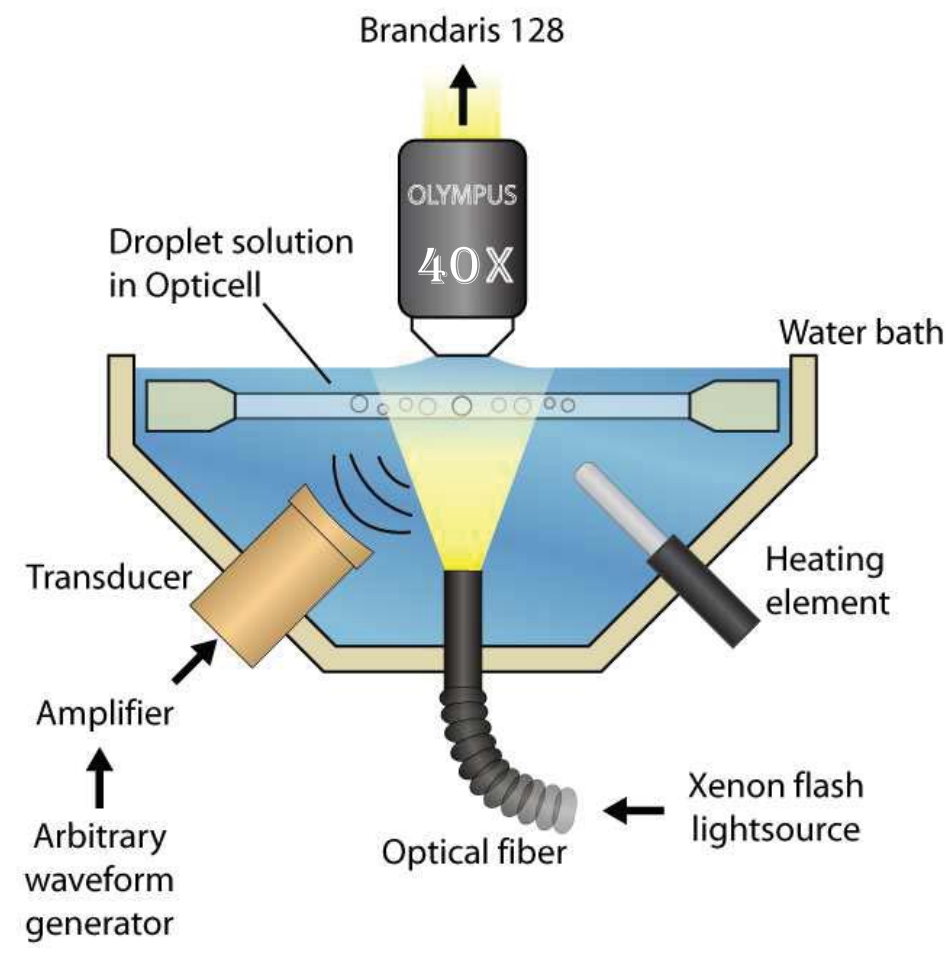

Figure 8.5: Schematic diagram of the experimental setup. Acoustic high intensity vaporization and low intensity characterization pulses were sent from the single element transducer. 
initial nucleation has expanded, secondary nucleation spots appear in the three nearby droplets. Two nuclei on each droplet initiate almost at the same time. The line passing through the two secondary nuclei of each droplet passes through the middle droplet as well. At $t=1.2 \mu$ s we see the further evolution of the system, the conversion of perfluoropentane liquid into vapor. It is also remarkable, that the three upper droplets undergo the secondary nucleation, but not the three lower ones. This can be attributed to the fact that the upper droplets have more neighboring droplets. Particularly, the upper droplet on a left has more neighbors than the upper droplet on the right. Such neighboring can be responsible for more violent vaporization of upper left droplet.

For single monodisperse droplets the vaporization thresholds for 1.3, 3.8 and $13.7 \mu \mathrm{m}$ radii were found to be $5.3 \pm 0.2,3.8 \pm 0.3$ and $3.08 \pm 0.07 \mathrm{MPa}$, respectively $\left(\mathrm{T}=33^{\circ} \mathrm{C}, \mathrm{N}=10\right.$ cycles, $\left.\mathrm{f}=3.5 \mathrm{MHz}\right)$. The error bar in determining the nucleation thresholds for monodisperse droplets is much smaller compared to polydisperse droplets [3]

\subsubsection{Symmetry of vaporization dynamics}

As previously observed in Chapter 3, vaporizing droplets can merge and fragment. Both the merging and the fragmentation of vaporizing monodisperse droplets have remarkable degree of symmetry. Fig. 8.7 shows the merging of two vaporizing monodisperse droplets with a radius of $3.8 \mu \mathrm{m}$. At $t=4.02 \mu \mathrm{s}$ the two vapor bubbles are merged into a single one. It subsequently fragmenting into 4 small attaching satellite vapor bubbles arranged symmetrically with respect to the line initially connecting the two droplets at the time $t=0 \mu \mathrm{s}$. It was also repeatedly observed that if one vaporizing monodisperse droplet fragments into multiple vapor bubbles, the nearby droplet has identical behavior. Whether droplets merge or not depends both on their size and initial separation (See Figs. 8.8 and 8.9). Fig. 8.8 shows the vaporization of the monodisperse droplets of the different sizes. Smaller droplets $\left(R_{d}=1.3 \mu \mathrm{m}\right)$ are attracted to each other as a result of secondary Bjerknes forces between the vapor bubbles [17] contained in them. Larger droplets $\left(R_{d}=13 \mu \mathrm{m}\right)$, on the other hand, stay in place due to drag. Fig. 8.9 shows the vaporization of the monodisperse droplets at different initial separation distances. Please note the order of magnitude difference between the interframe time intervals. The first couple, which have a larger initial separation distance, are not yet merged at $0.98 \mu \mathrm{s}$, while the ultrasound is still forcing them. They are merged only a few microseconds later due to their radial expansion. However the second 

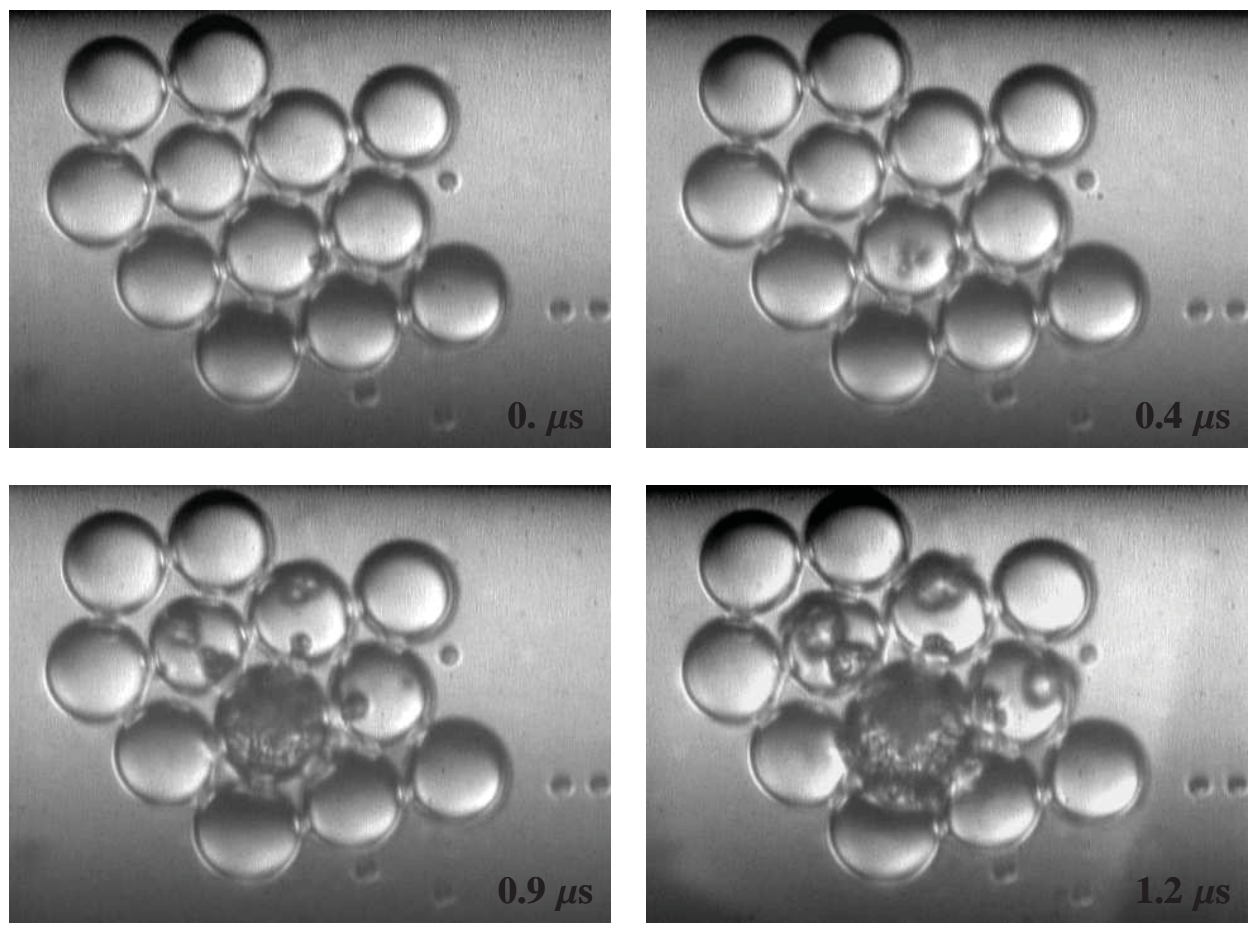

Figure 8.6: Vaporization of clusters of monodisperse droplets with a radii of $13.7 \mu \mathrm{m}$. The vaporization is triggered at $3.5 \mathrm{MHz}$ ultrasound with 10 cycles of $3.7 \mathrm{MPa}$ peak negative pressure amplitude. 


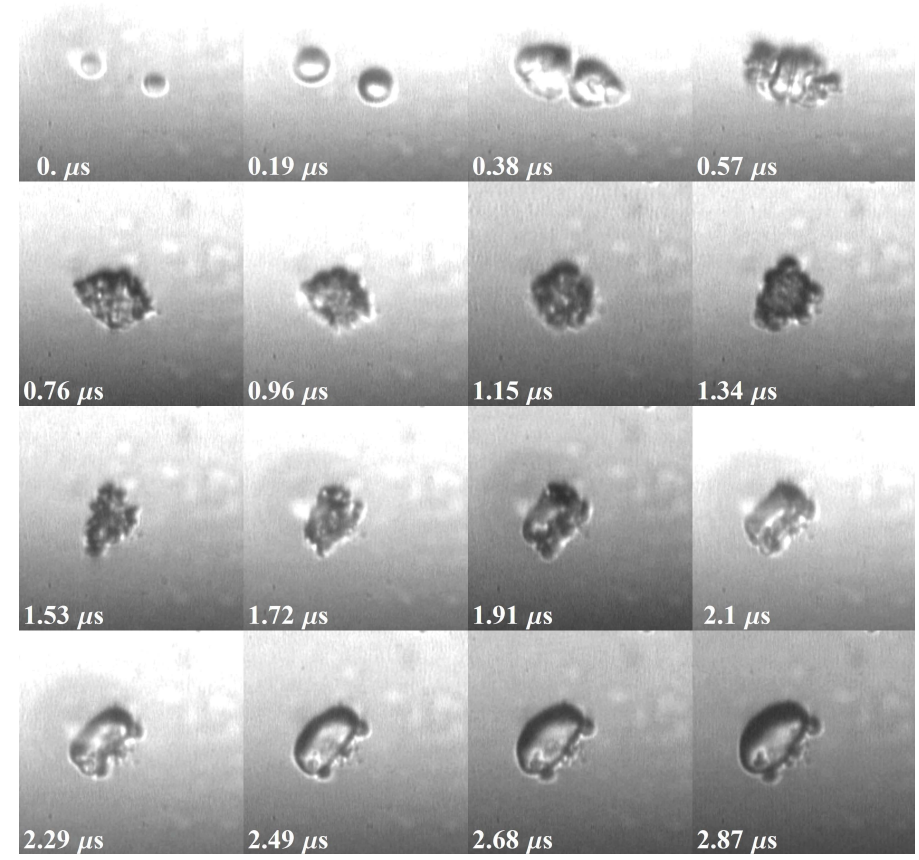

Figure 8.7: Consecutive images of vaporization of $3.8 \mu \mathrm{m}$ radius droplets. Vaporization dynamics and fragmentation of resulting bubbles is symmetric with respect to the line, connecting the initial position of the two droplets.

couple is merged already at $0.48 \mu \mathrm{s}$. Note also the symmetry of merging of the first monodisperse droplet couple. The conclusion of this set of experiments is that the ultrasound forcing brings a higher degree of randomness into the vaporization dynamics, while phase conversion without ultrasound forcing is predictable and smooth.

\subsubsection{Activation below boiling point}

In this section we aim to visualize how perfluoropentane droplets below their boiling point react to the exposure to focused ultrasound. For this purpose we perform a series of experiments at different ambient temperatures $T_{\infty}$, namely at $11.8,15.9,19.0,23.8,29.5,33.4,37.2$ and $41.8^{\circ} \mathrm{C}$. The boiling temperature of perfluoropentane is $T_{b}=29^{\circ} \mathrm{C}$. Fig. 8.10 shows the radial dynamics of the vapor bubble growth with time for different ambient temperatures above and 


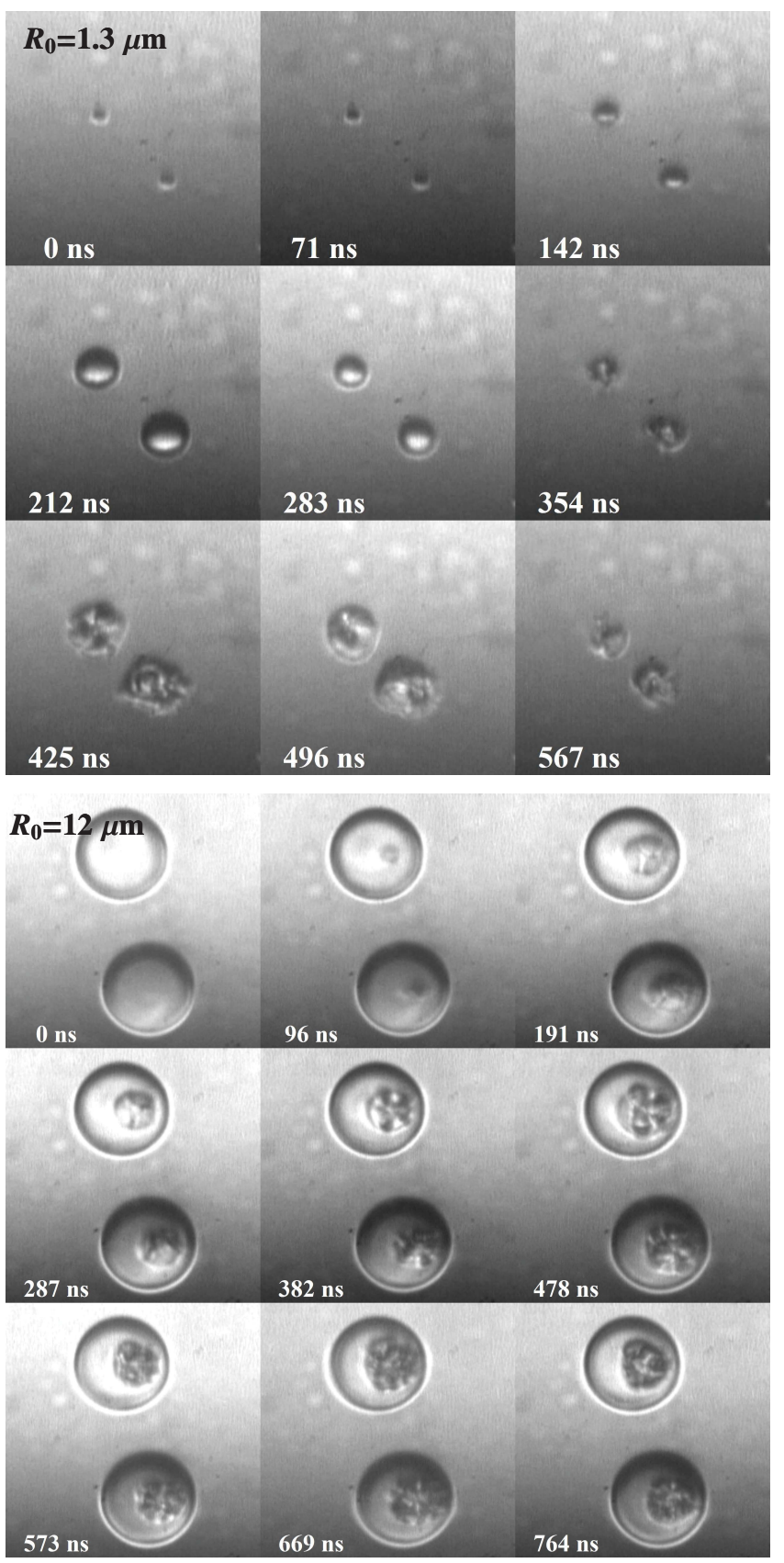

Figure 8.8: Set of consecutive images of vaporization of monodisperse droplets of sizes of 1.3 and $12 \mu \mathrm{m}$, triggered at $3.5 \mathrm{MHz}$ ultrasound with 10 cycles of 4.6 and 3.0 MPa peak negative pressure amplitudes, respectively. 

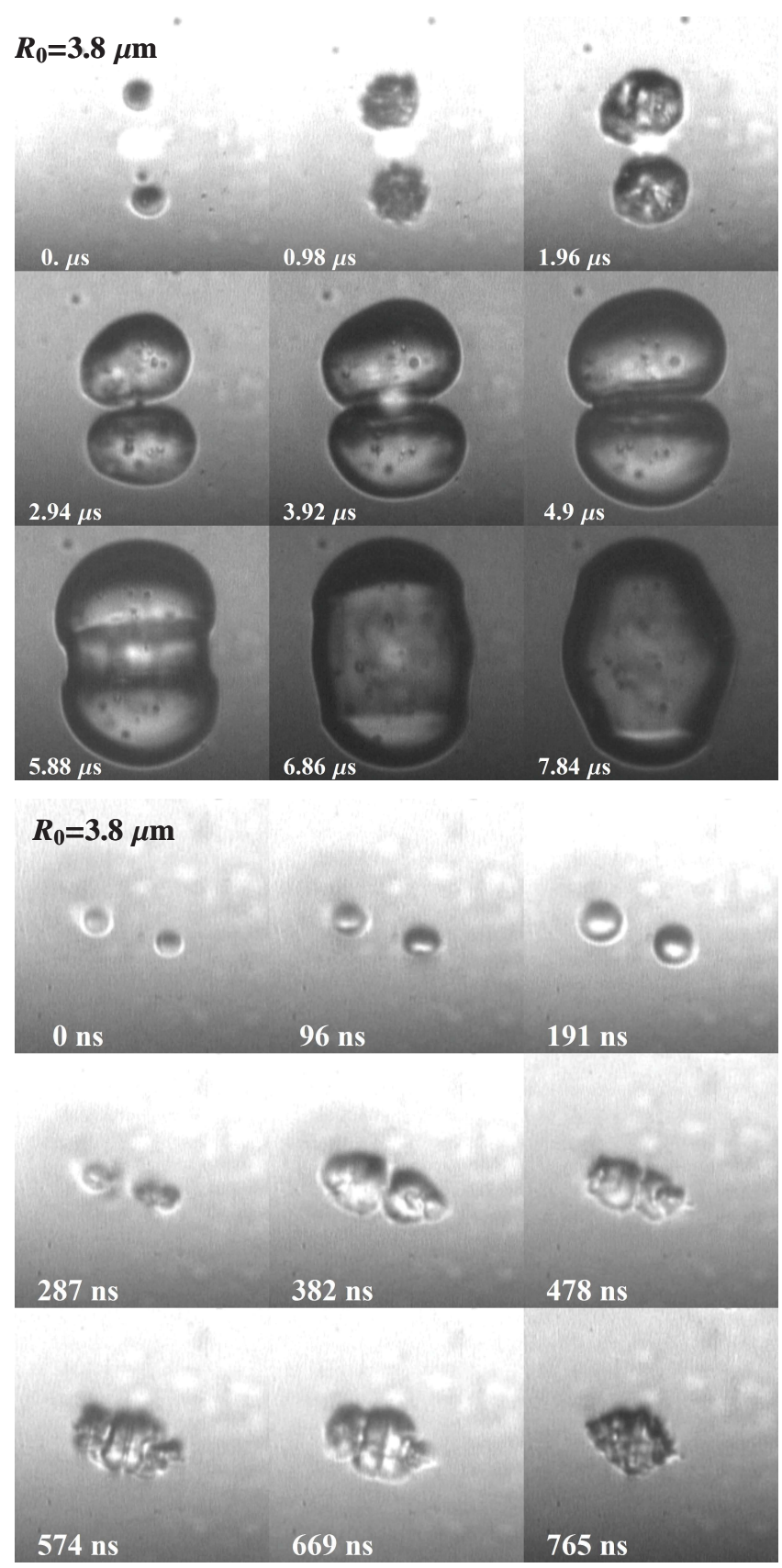

Figure 8.9: Set of consecutive images of vaporization of monodisperse droplets of sizes of $3.8 \mu \mathrm{m}$ triggered at a frequency of $3.5 \mathrm{MHz}$ with 10 cycles of $3.7 \mathrm{MPa}$ peak negative pressure amplitude at different initial separation distances. Note the order of magnitude difference between the time scales. 
below boiling point. Each black line correspond to an effective radius of vapor bubble, determined by the outer droplet radius using the mass conversion principle. The black lines represent data collected from different droplets of the same size. The red lines show the theory of heat transfer growth enhanced by the ultrasound forcing discussed in Chapter 5 as expressed by Eq. 5.8. The theory cannot be applied in the case of bubble growth below boiling point. The radius-time growth curve at $T_{\infty}-T_{b}=12.8 \mathrm{~K}$ has the highest level of repeatability. However, decreasing the temperature makes the vaporization process slower. Smaller bubbles are more susceptible to more pronounced interaction with the forcing ultrasound, thus the vaporization at lower temperatures has higher degree of randomness. Starting at $T_{\infty}-T_{b}=-5.2 \mathrm{~K}$ growing bubble can even fragment into 3-4 smaller bubbles. Due to the slow pace of bubble growth below the boiling point the second appearing nuclei on the distal side of the droplet (more on secondary nucleation see Chapter 4) may not merge with the primary nucleus at the proximal side (Fig. 8.11). Such a system of two bubbles interact with ultrasound in a complex way. Thus, these effects make the radial time dynamics below the boiling point poorly repeatable (Fig. 8.10 at $\mathrm{T}_{\infty}-\mathrm{T}_{\mathrm{b}}=-5.2,-10.0,-13.1,-17.2 \mathrm{~K}$ ).

To quantify the dependence on the radial dynamics of the bubble expansion on temperature and to minimize the influence of randomness, sets of data taken at the same temperature were averaged. Fig. 8.12 shows the average vapor bubble radius as a function of ambient temperature at 3,5 and $7 \mu \mathrm{m}$ after the initiation of nucleation. One can see the clear difference of behavior of bubble expansion dynamics as a function of temperature. Below the boiling point the bubble grows slowly on time after elapsing of $3 \mu \mathrm{s}$. Most of bubble growth happens within the first microsecond. Please, also note, that below boiling point the radius of bubble at 3,5 and $7 \mu \mathrm{m}$ after the initiation of nucleation does not depend on the ambient temperature. Above boiling point after $t=3 \mu$ s the bubble is growing further on time and is linearly faster when the ambient temperature temperature is larger.

In order to further investigate the origin of the bubble growth dynamics below boiling point we insonified bubbles with the same 4 cycles ultrasound pulse 5 times after each $100 \mathrm{~ms}$. The example of the first insonification with 10 cycles we already saw in Fig. 8.11. Fig. 8.13 shows the result of the second insonification $100 \mathrm{~ms}$ later. We see that after $100 \mathrm{~ms}$ the bubble inside the droplet is still persistent. It weakly interacts with the ultrasound wave due to its relatively large off-resonant size. Fig. 8.14 shows the radius time dynamics of the bubble during the second (indicated as $t_{0}=0.1 \mathrm{~s}$ ), third (indicated as 

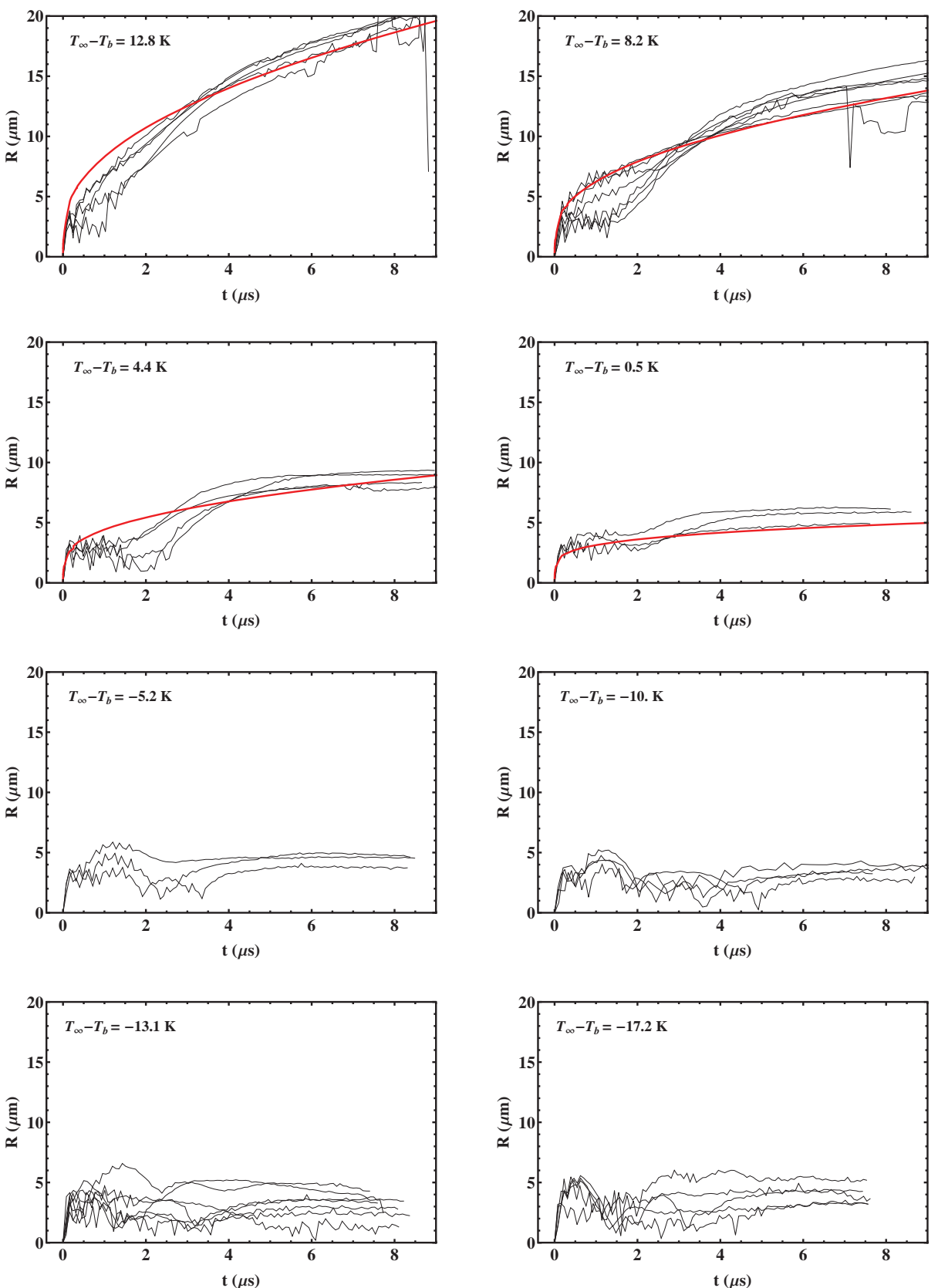

Figure 8.10: Radius time dynamics of the vapor bubble recordered at different ambient temperatures $\mathrm{T}_{\infty}$ Perfluoropentane boiling temperature $\mathrm{T}_{\mathrm{b}}=29^{\circ} \mathrm{C}$. The vaporization is triggered at a frequency of $5.0 \mathrm{MHz}$ with 4 cycles of $3.2 \mathrm{MPa}$ peak negative pressure amplitude. Red lines are the theory of heat transfer growth enhanced by the ultrasound forcing discussed in Chapter 5 expressed as Eq. 5.8. The black lines represent the experimental data taken with droplets of the same radius, $R_{d}=7.4 \mu \mathrm{m}$. 


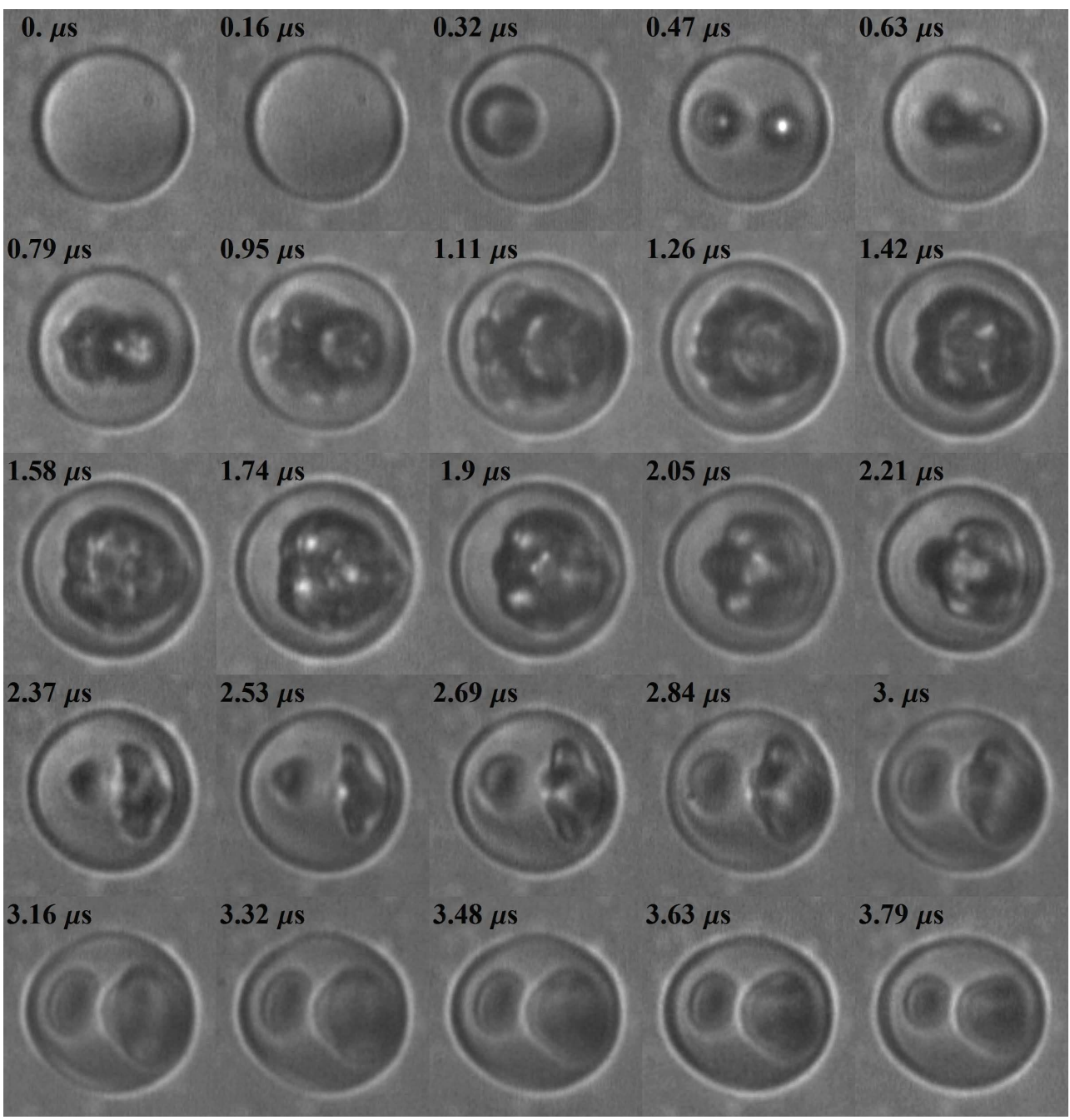

Figure 8.11: Vaporization dynamics of $\mathrm{R}_{\mathrm{d}}=7.4 \mu \mathrm{m}$ droplet below its boiling point at $\mathrm{T}_{\infty}-\mathrm{T}_{\mathrm{b}}=-13.1 \mathrm{~K}$. The vaporization is triggered at a frequency of $5.0 \mathrm{MHz}$ with 4 cycles of $3.2 \mathrm{MPa}$ peak negative pressure amplitude. 


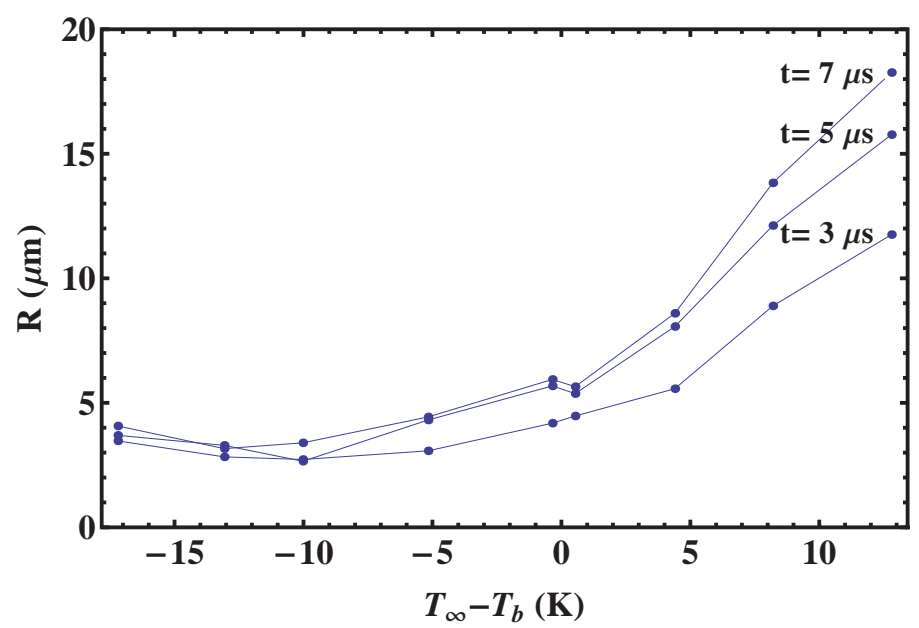

Figure 8.12: Averaged vapor bubble radius as a function of the temperature relative to the boiling point at 3, 5 and $7 \mu$ s after the initiation of nucleation. Initial droplet radius $\mathrm{R}_{\mathrm{d}}=7.4 \mu \mathrm{m}$.

$t_{0}=0.2 \mathrm{~s}$ ), fourth (indicated as $t_{0}=0.3 \mathrm{~s}$ ) and fifth (indicated as $t_{0}=0.4 \mathrm{~s}$ ) insonification. After each $100 \mathrm{~ms}$ the initial bubble radius is steadily increasing due to the rectified gas diffusion phenomenon when ultrasound forces the system. Oscillations during $2 \mu<t-t_{0}<3 \mu$ s are due to ultrasound forcing at a frequency of $5 \mathrm{MHz}$. This forcing makes the bubble to temporarily shrink, however the net amount of gas inside the bubble increases due to the rectified gas diffusion phenomenon, which then leads to oscillations at bubble eigenfrequency when ultrasound is turned OFF.

The fact that the bubble is still persistent after $100 \mathrm{~ms}$ while being below boiling point and with a volume similar to the volume of the initial droplet suggests that the bubble contains a large amount of diffused gas from the droplet medium which prevents the vapor from recondensing.

\subsection{Conclusion}

In this study we show that the vaporization dynamics has an exceptional degree of repeatability with monodisperse droplets compared to experiments using polydisperse ones. We found that the vaporization thresholds for 13.7, 3.8 and $1.3 \mu \mathrm{m}$ droplets were $3.08 \pm 0.07,3.8 \pm 0.3$ and $5.3 \pm 0.2 \mathrm{MPa}$ re- 


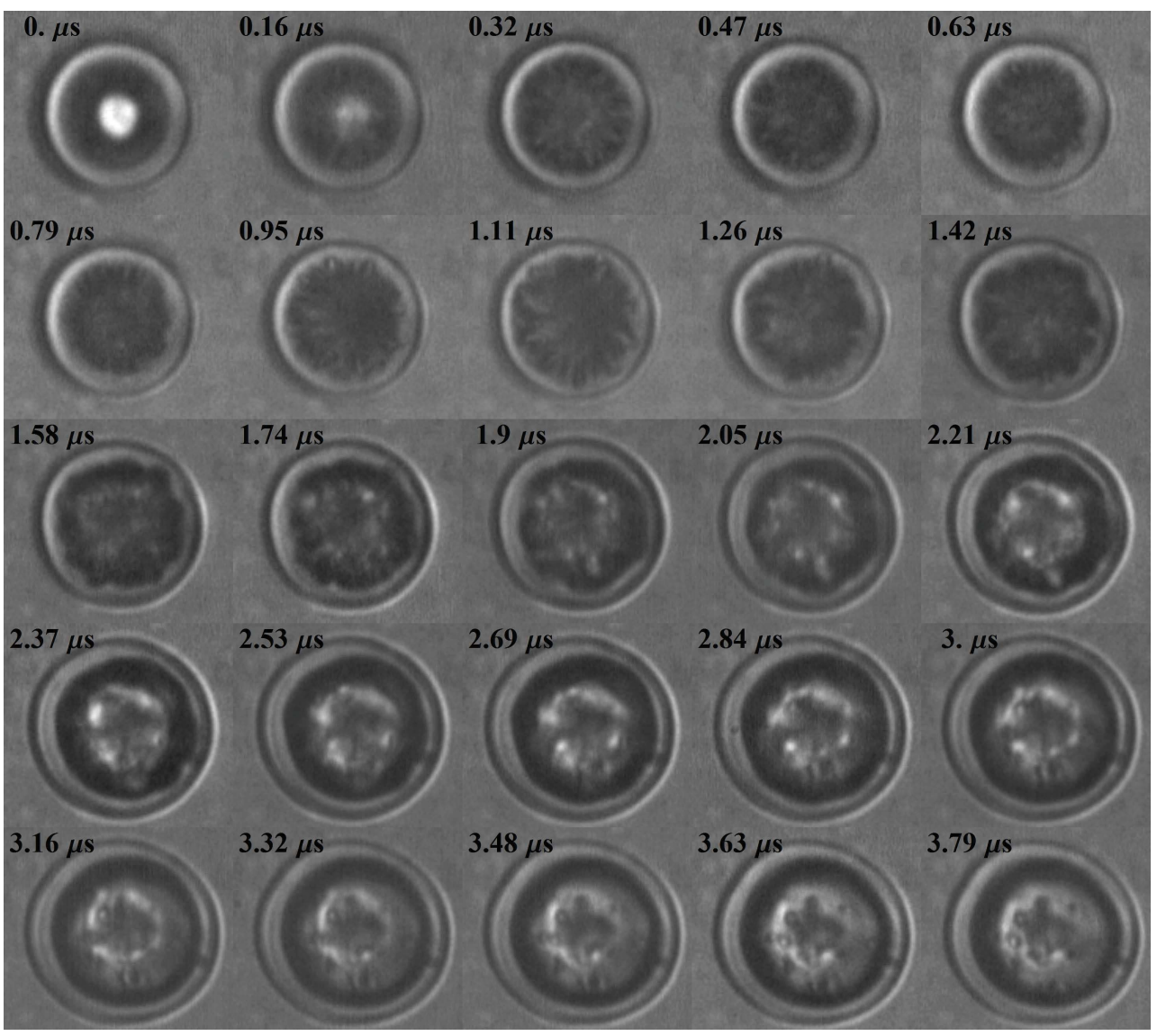

Figure 8.13: Interaction of created droplet-bubble system with ultrasound during the second insonification $100 \mathrm{~ms}$ after the first insonification. $\mathrm{T}_{\infty}-\mathrm{T}_{\mathrm{b}}=-13.1 \mathrm{~K}$. The droplet-bubble system is forced at a frequency of $5.0 \mathrm{MHz}$ with 4 cycles of $3.2 \mathrm{MPa}$ peak negative pressure amplitude. 


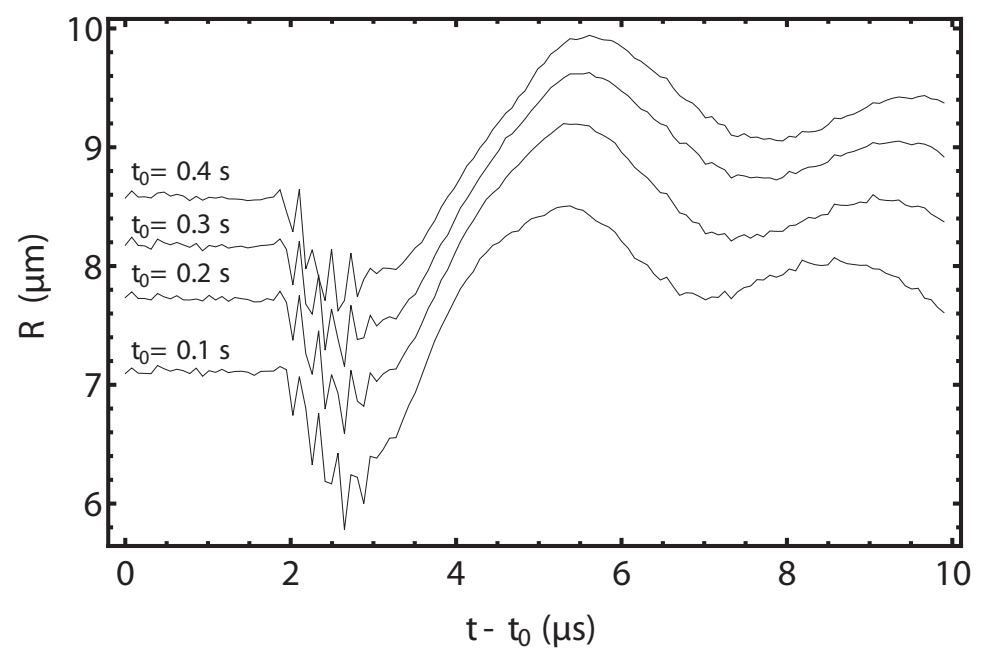

Figure 8.14: The radius time dynamics of bubble during the second (indicated as $\mathrm{t}_{0}=0.1 \mathrm{~s}$ ), third (indicated as $\mathrm{t}_{0}=0.2 \mathrm{~s}$ ), fourth (indicated as $\mathrm{t}_{0}=0.3 \mathrm{~s}$ ) and fifth (indicated as $\mathrm{t}_{0}=0.4 \mathrm{~s}$ ) insonification. The droplet-bubble system is forced at a frequency of $5.0 \mathrm{MHz}$ with 4 cycles of $3.2 \mathrm{MPa}$ peak negative pressure amplitude.

spectively at $33^{\circ} \mathrm{C}$ ambient temperature using a 10 cycles excitation burst at 3.5 $\mathrm{MHz}$ central transducer frequency. The nucleation and radial expansion dynamics is symmetric, controllable, and reproducible and the vaporization threshold was found to lie within a pressure range as low as $70 \mathrm{kPa}$, while the standard deviation of the step response agrees very well with that of the droplet size. Such a fine-tuned step-response is highly desirable for clinical applications. It was demonstrated that with the current nanochannel design droplets can be probably produced as small as $260 \mathrm{~nm}$, which are one of the smallest monodisperse PFP droplets reported to date. The initiation of vaporization was imaged at a range of temperatures below its bolinig point. It was shown that below its boiling point the droplet-bubble complex is persistent for more than $500 \mathrm{~ms}$ and that the radius of the inner bubble can be controlled by repetitive insonifications due to rectified gas diffusion phenomena.

\section{References}

[1] N. Reznik, R. Williams, and P. Burns, "Investigation of vaporized submicron perfluorocarbon droplets as an ultrasound contrast agent", Ultra- 
sound Med. Biol. 37, 1271-1279 (2011).

[2] N. Reznik, M. Seo, R. Williams, E. Bolewska-Pedyczak, M. Lee, N. Matsuura, J. Gariepy, F. Foster, and P. Burns, "Optical studies of vaporization and stability of fluorescently labelled perfluorocarbon droplets", Phys. Med. Biol. 57, 7205-7217 (2012).

[3] N. Reznik, O. Shpak, E. C. Gelderblom, R. Williams, N. de Jong, M. Versluis, and P. N. Burns, "The efficiency and stability of bubble formation by acoustic vaporization of submicron perfluorocarbon droplets", Ultrasonics 53, 1368-1376 (2013).

[4] O. Shpak, T. Kokhuis, Y. Luan, D. Lohse, N. de Jong, B. Fowlkes, M. Fabiilli, and M. Versluis, "Ultrafast dynamics of the acoustic vaporization of phase-change microdroplets", J. Acoust. Soc. Am. 134, 1610-1621 (2013).

[5] O. Shpak, L. Stricker, M. Versluis, and D. Lohse, "The role of gas in ultrasonically driven vapor bubble growth", Phys. Med. Biol. 58, 2523 2535 (2013).

[6] O. Shpak, M. Verweij, R. Vos, N. de Jong, D. Lohse, and M. Versluis, "Acoustic droplet vaporization is initiated by superharmonic focusing", Proc. Natl. Acad. Sci. 111, 1697-1702 (2014).

[7] O. D. Kripfgans, J. B. Fowlkes, D. L. Miller, O. P. Eldevik, and P. L. Carson, "Acoustic droplet vaporization for therapeutic and diagnostic applications”, Ultrasound Med. Biol. 26, 1177-1189 (2000).

[8] O. Couture, M. Faivre, N. Pannacci, A. Babataheri, V. Servois, P. Tabeling, and M. Tanter, "Ultrasound internal tattooing", Med. Phys. 38, 1116-1123 (2011).

[9] T. Segers and M. Versluis, "Acoustic bubble sorting for ultrasound contrast agent enrichment", Lab on a Chip 14, 1705-1714 (2014).

[10] P. S. Sheeran, V. P. Wong, S. Luois, R. J. McFarland, W. D. Ross, S. Feingold, T. Matsunaga, and P. Dayton, "Decafluorobutane as a phase-change contrast agent for low-energy extravascular ultrasonic imaging.", Ultrasound Med. Biol. 37, 1518-1530 (2011). 
[11] P. S. Sheeran, S. Luois, P. A. Dayton, and T. O. Matsunaga, "Formulation and acoustic studies of a new phase-shift agent for diagnostic and therapeutic ultrasound", Langmuir 27, 10412-10420 (2011).

[12] P. S. Sheeran, S. H. Luois, L. B. Mullin, T. O. Matsunaga, and P. A. Dayton, "Design of ultrasonically-activatable nanoparticles using low boiling point perfluorocarbons", Biomaterials 33, 3262-3269 (2012).

[13] T. D. Martz, P. S. Sheeran, D. Bardin, A. P. Lee, and P. A. Dayton, "Precision manufacture of phase-change perfluorocarbon droplets using microfluidics", Ultrasound Med. Biol. 37, 1952-1957 (2011).

[14] M. Seo and N. Matsuura, "Monodisperse, submicrometer droplets via condensation of microfluidic-generated gas bubbles", Small 8, 27042714 (2012).

[15] L. Shui, A. van den Berg, and J. C. T. Eijkel, "Scalable attoliter monodisperse droplet formation using multiphase nano-microfluidics", Microfluid. and Nanofluid. 11, 87-92 (2011).

[16] C. Chin, C. Lance, J. Borsboom, F. Mastik, M. Frijlink, N. de Jong, M. Versluis, and D. Lohse, "Brandaris 128: A digital 25 million frames per second camera with 128 highly sensitive frames", Rev. Sci. Instrum. 74, 5026-5034 (2003).

[17] V. F. K. Bjerknes, Fields of Force (Columbia University Press) (1906). 


\section{9 \\ Conclusions and Outlook}

This thesis describes the physical properties of the dynamics of droplet vaporization under the influence of ultrasound. Here, we list the major findings from our studies.

In Chapter 2 of this thesis we explain the basics of the bubble dynamics, described with the Rayleigh-Plesset equation, its resonance frequency, damping and quality factor. We show the elegant calculation of the above characteristics in case of small amplitude oscillations by linearizing the equations. In addition the ultrasound propagation is introduced. We explain the speed of sound, the nonlinearity and attenuation terms. We discuss the bubble ultrasound scattering and how it depends on the wave-shape of the incident wave. Finally we introduce the droplets interaction with ultrasound. We explain the ultrasound focusing concept on a droplets sphere, droplet shaking due to media compressibility, and droplet phase-conversion dynamics.

In Chapter 3 we observe the vaporization of submicron PFC droplet for the first time at a nanoseconds timescale. This examination of the droplets revealed phenomena that could not be detected at lower imaging frame rates. The results have shown effects of shell material retention by the bubbles after vaporization. We estimated that the efficiency of conversion of droplets into bubbles when exposed to an acoustic pulse within the diagnostic range is at least $10 \%$. In this chapter we also show that submicron droplets may undergo 
coalescence or fragmentation during the initial oscillations following droplet vaporization. Bubble coalescence, effectively increasing the shell material concentration on the bubble surface, increases the chances of bubble survival. An increase of the initial droplet concentration, leading to a reduced interdroplet separation distances, hence, is beneficial for the production of stable microbubbles. Thus, the efficiency of initial droplet vaporization, and the efficiency of stable bubble production may be very different, depending on the excitation conditions.

In Chapter 4 is presented a study into the origin of acoustic droplet vaporization mechanism. The Brandaris ultra high-speed camera is used to investigate nucleation within the droplets in the parameter space of the droplet size and acoustic parameters such as pressure amplitude and frequency. We showed that ADV is initiated by the focusing of the superharmonics of a nonlinearly distorted acoustic wave within the droplet. In the parameter range considered, the focusing leads to up to a ten-fold increase of the incident peak negative pressure in a well-defined focusing spot. The location of the focusing spot as well as the focusing strength depends on the frequency of the incident wave and on the size of the droplet. A higher frequency and a larger droplet radius, i.e. a smaller wavelength compared to the droplet size, leads to a more pronounced focusing effect and consequently leads to smaller pressure amplitudes required for activation. Nonlinear propagation is shown to have a crucial role in ADV. It causes distortion of the pressure waveform and the resulting superharmonics are highly focused within the spherical droplet. Thus, the transducer geometry, as well as the parameters for nonlinearity of the propagating medium have an effect on the focusing strength and the position of focusing.

In Chapter 5 the Brandaris ultrahigh-speed camera is used to investigate the radial dynamics of acoustic droplet vaporization. Acoustic microdroplet vaporization was imaged for the first time at a nanoseconds time scale with a sampling rate of 3-5 images per cycle, being able to capture prenucleation, nucleation, during ultrasound and post ultrasound, all for a single vaporization event. With the proposed method of pressure restoration it was shown that the inception of nucleation occurs during the peak negative half cycle of ultrasound and slightly shifted towards the end of it. Following nucleation, the physics of the vapor bubble expansion is shown to be heat transfer limited. The vapor bubble expansion was observed to have two components, one monotonic with a typical velocity of expansion of 5-10 m/s and a second oscillatory component with the typical amplitude of $1.2 \mu \mathrm{m}$ associated with the 
applied ultrasound. The vapor bubble growth is increased by acoustic activation and through a simple physical model it was shown to be the result of rectified heat transfer when the ultrasound is forcing the vapor bubble.

In Chapter 6 we develop a model, describing the evolution of an ultrasound driven vapor bubble, which includes both thermal diffusion and mass diffusion of gas (i.e. air) inside the liquid. Simulations underlined the fundamental role of gas diffusion during acoustic forcing. Though the bubble dynamics is entirely governed by evaporation and condensation processes, as the ratio between gas and vapor mass is $\mathrm{m}_{\mathrm{G}} / \mathrm{m}_{\mathrm{V}} \sim 10^{-2}$, a minimum amount of gas ( $\sim 10 \%$ of the air solubility in PFP) is necessary in order to allow the bubble to survive the first collapse. When no driving is present, inclusion of the gas diffusion has no influence on the bubble growth.

In Chapter 7 we investigated the acoustic characteristics of vaporized submicron droplets at clinically relevant timescales. It was shown that following vaporization with ultrasound, the newly created microbubbles oscillate nonlinearly and exhibit coating material-induced effects on their oscillations that can be described by the de Jong coated bubble dynamics model. The coating material on the surface of the bubbles induces a shift in the resonance frequency and a substantial amount of damping due to shell viscosity. Unlike lipid-coated ultrasound contrast agents, the fluorosurfactant coated bubbles used in this study do not exhibit buckling behavior, and tend to feature expansion-dominated oscillations for smaller bubbles. At the same time, it was shown that vaporized PFC droplets scatter ultrasound in a manner similar to the currently clinically used microbubble contrast agents. These results suggest that stable vaporized perfluorocarbon droplets possess the adequate acoustic characteristics to support their potential use as clinical ultrasound contrast agents.

In the final Chapter 8 we fabricate monodisperse droplets using microfluidic chip technology. We show that the vaporization dynamics has an exceptional degree of repeatability for monodisperse droplets as compared to polydisperse droplets. The nucleation and radial expansion dynamics is symmetric, controllable, and reproducible. The vaporization threshold was found to lie within a pressure range as low as $70 \mathrm{kPa}$, while the standard deviation of the step response agrees very well with that of the droplet size. Such a fine-tuned step-response is highly desirable for clinical applications. It was demonstrated that with the current micro-nanochannel chip design droplets can be produced as small as $260 \mathrm{~nm}$ in radius, which are one of the smallest monodisperse PFP droplets reported to date. The initiation of vaporization 
was imaged at a range of temperatures below and above the boiling point. It was show that below boiling point the droplet-bubble complex is persistent for more than $500 \mathrm{~ms}$ and that the radius of the inner bubble can be controlled by the secondary insonifications deploying a rectified gas diffusion phenomena.

The work presented here has answered a number of important questions in regards to physical mechanism of droplet vaporization, accounting for both thermodynamic and acoustic parameters. We have the confidence that the knowledge that was gained within the present work could improve the efficiency of acoustic droplet vaporization for both imaging and therapeutic applications. We opened up the ways of minimization of the vaporization threshold in order to maximize bubble production with ultrasound. However, a considerable amount of not addressed issues remains. A large amount of work is yet to be done in order to develop the droplets as clinically acceptable therapeutic and imaging agents. Specifically, directions for further work should be focused on a number of aspects:

- Droplet vaporization close to cells - the process of ADV mechanically releases the drug phase encapsulated within a double emulsion, a process which is poorly understood. Further work is necessary in order to quantify the shear stresses on the drug phase during the release process as well as determine the extent of nebulization using high-speed imaging. ADV not only releases drugs, it can also transiently permeabilizes the cell membrane to enhance drug uptake. Fluorescent highspeed imaging of cells adjacent to vaporizing droplets can illustrate the bioeffects of ADV as well as the real-time efficiency of drug penetration into the cell.

- Theory of acoustic droplet vaporization below boiling point - even though the important role of gas diffusions is evident in acoustic droplet vaporization below its boiling point, the theoretical modeling and numerical simulations are required.

- Design of dual or multiple frequency transducer - using dual or multiple frequency transducers, the amplitudes and phases of the transmit waves can be optimized to have maximal constructive interference within the droplets to maximize the focusing strength at any given acoustic input pressure.

- Droplet stability and biocompatibilty - different droplet preparation protocols are to be investigated in order to create droplet emulsions that 
have the ability to both produce stable bubbles and also limit bubble growth following vaporization. This includes the design of droplets by mixing different liquids with different physical properties which allows to vary the acoustic impedance by a change of the density and speed of sound to maximize the focusing effect. Droplet emulsion biocompatibility is to be studied for future safe application of droplets in the clinical settings in regard of their safety.

The use of vaporized PFC droplets as contrast agents is a relatively new approach. Vaporizable PFC droplets have the potential advantages compared to the currently available microbubble contrast agents. In this thesis we have established the required physical aspects of droplet vaporization necessary to make them efficient imaging and therapeutic agent. We believe that with the rest of the necessary conditions met, the droplets have the potential to become a principally new tool for cancer detection and treatment with ultrasound. They would significantly increase the potential of medical ultrasound to successfully advance the medicine. 


\section{Samenvatting}

Dit proefschrift beschrijft de fysica van de verdamping door ultrageluid van metastabiele perfluorokoolwaterstof microdruppels met een kookpunt lager dan hun omgevingstemperatuur.

$\mathrm{Na}$ een korte inleiding over druppels, bellen en ultrageluid beschrijven we in hoofdstuk 2 de fysische interacties van druppels, bellen en ultrageluid. We beginnen met de fysica van een oscillerende gasbel in termen van de veelgebruikte Rayleigh-Plesset vergelijking. Een linearisatie van deze vergelijking, voor kleine trillingen van de bel, laat op een elegante manier is hoe de resonantiefrequentie en de demping van het systeem te vinden is in hoofdstuk 2 wordt ook de propagatie van ultrageluid beschreven. Het omvat een beschrijving van de geluidssnelheid, van de niet-lineaire propagatie en de vorming van een schokgolf en de verzwakking. Vervolgens wordt besproken hoe ultrageluid verstrooit aan een bel en hoe dit afhangt van de vorm van de golf. Tot slot beschrijven we in detail de interactie van een geluidsgolf en een druppel, met inbegrip van de mogelijkheid tot het focusseren van de golf in de druppel, het oscilleren van een druppel onder invloed van het ultrageluid als ook de door het ultrageluid genduceerde verdamping van de druppels.

In hoofdstuk 3 laten we voor het eerst op nanoseconden tijdschaal zien hoe het verdampingsproces van druppels precies plaats vindt. De opnamen laten zien dat het schilmateriaal van de druppel achter kan blijven op de dampbel nadat de druppel verdampt is. Een afschatting van de conversie van druppels naar bellen leert dat die ongeveer tien procent is bij diagnostische akoestische drukken. Verder laten we zien dat submicrometer druppels kunnen coalesceren en fragmenteren tijdens de eerste oscillaties na de vorming van de dampbel. De coalescentie van bellen verhoogt de effectieve oppervlakteconcentratie van het schilmateriaal en daarmee de levensduur van de bel. Het is daarom zeer waarschijnlijk dat een hoge druppelconcentratie voordelig is voor de vorming van stabiele microbellen. Echter, de verdamping van druppels die zich op een kleine afstand van elkaar bevinden wordt bemoeilijkt 
door het afschermen van de geluidsgolf door de naburige druppels. Dus, de efficintie waarmee druppels verdampt kunnen worden en die van het mechanisme waarmee stabiele bellen gevormd kunnen worden is waarschijnlijk zeer verschillend.

In hoofdstuk 4 presenteren we een studie naar de onderliggende fysische mechanismen van de initiatie van de verdamping van druppels door ultrageluid. De Brandaris hogesnelheidscamera is gebruikt om de positie van nucleatiekernen in de druppel zichtbaar te maken als functie van de druppelgrootte en de akoestische parameters, zoals die van de druk en de frequentie. We hebben kunnen laten zien dat de druppelverdamping wordt genitieerd door het focusseren van hogere harmonischen van de inkomende ultrageluidsgolf door niet-lineair vervorming op weg naar de druppel. Het focusseren van de energie leidt tot een akoestische negatieve druk in het focus dat wel tien keer hoger is dan de amplitude van de inkomende geluidsgolf. De positie van het focus alsmede de druk in het focus hangen sterk af van de frequentie van de akoestische golf en de grootte van de druppel. Naarmate de golflengte van het geluid kleiner wordt, ten opzichte van de afmeting van de druppel, treed er een hogere mate van focussering op wat leidt tot het gunstige effect dat er een lagere druk gebruikt kan worden om de druppel te verdampen. De nietlineaire propagatie van het ultrageluid is daarbij essentieel in de verdamping van druppels omdat de gevormde hogere harmonischen een golflengte hebben van een grootte als die van de druppels en dus efficint in de druppel kunnen worden afgebogen door het druppeloppervlak. De transducergeometrie alsmede de niet-lineare propagatie parameters van het medium rondom de druppel hebben een belangrijk effect op de amplitude en de positie van het focus in de druppel.

Hoofdstuk 5 laat de resultaten zien van het onderzoek dat we deden met onze supersnelle camera naar druppelverdamping. Het visualiseren van de druppelverdamping op nanosecondenprecisie maakte het mogelijk het gehele nucleatieproces in beeld te brengen. Vanuit de hogesnelheidsbeelden wordt de fase van de inkomende akoestische golf berekend en daarmee is bepaald dat het verdampingsproces geiniteerd kan worden tijdens of vlak na het passeren van de laagste druk van het ultrageluid. De mate waarin de gevormde dampbel na nucleatie groeit blijkt te worden gelimiteerd door de warmtediffusie. De groeisnelheid van de dampbel heeft typisch twee componenten waarvan de eerste monotoon stijgt met een snelheid van 5 tot $10 \mathrm{~m} / \mathrm{s}$. De tweede component oscilleert met een typische amplitude van 1 micrometer en is aangedreven door het inkomende ultrageluid. De groeisnelheid van de dampbel als 
geheel blijkt groter te worden als deze akoestisch wordt aangedreven. Een simpel model laat zien dat dit veroorzaakt wordt door verschillende cycli van warmtediffusie.

In hoofdstuk 6 presenteren we een model voor de groei van een door ultrageluid aangedreven microbel, waarin de effecten van zowel thermische diffusie, alsmede diffusie van het gas in de vloeistof opgenomen zijn. Simulaties gebaseerd op dit model onderstrepen de belangrijke rol van gasdiffusie tijdens het akoestisch aandrijven van de bel. Ondanks het feit dat de dynamica van de bel volledig wordt beheerst door verdamping en condensatie, is er een minimale hoeveelheid gas nodig (ca. 10\% van de totale opgeloste hoeveelheid lucht in het perfluoropentaan) om de bel de eerste compressiefase te laten overleven, vanwege de wetmatige verhouding tussen de hoeveelheid gas en damp. Zonder akoestische aandrijving van de bellen heeft de gasdiffusie geen enkele invloed op de groei van de bellen.

In hoofdstuk 7 beschrijven we de akoestische eigenschappen van verdampte submicron druppels op klinisch relevante tijdschalen. We laten zien dat de dampbellen, die ontstaan na het verdampen van de druppels door het ultrageluid, niet-lineair oscilleren, en dat het gedrag bepaald wordt door de omhullende schil. Het schilmateriaal op het oppervlak van de bellen veroorzaakt bovendien een verschuiving van de resonantiefrequentie van de bellen, alsmede een flinke demping door de viscositeit van het schilmateriaal. In tegenstelling tot de gebruikelijke microbellen die gecoat zijn met lipiden, zijn de bellen uit deze studie omgeven door fluorhoudende oppervlakte-actieve stoffen. Daardoor vertonen ze niet het typische gedrag waarbij de schil kreukelt bij compressie van de bel, maar worden de oscillaties van de bellen voornamelijk gedomineerd door expansie. Ondanks dit verschil in gedrag, laten we zien dat de verdampte PFC druppels het ultrageluid op dezelfde manier verstrooien als de klinisch gebruikte contrastmiddelen voor ultrageluid. Deze resultaten laten dus zien dat stabiele microbellen, geproduceerd door verdamping van PFC microdruppels met ultrageluid, de juiste akoestische eigenschappen bezitten voor het gebruik als contrastmiddel voor ultrageluid.

In het laatste hoofdstuk, hoofdstuk 8 , beschrijven we het maken van monodisperse druppels met behulp van microfludische chips. We laten zien dat de reproduceerbaarheid van de wijze van verdamping van monodisperse druppels veel hoger is dan die van polydisperse druppels. De nucleatie en radile expansie zijn symmetrisch om de lijn van ultrageluid propagatie en bovendien zijn ze controleerbaar en reproduceerbaar. De drempelwaarde voor verdamping ligt onder de $70 \mathrm{kPa}$, terwijl de foutenmarge van de staprespons marginaal 
klein is en ongeveer overeenkomt met de spreiding in de grootte van de druppels, wat zeer wenselijk is voor klinische toepassingen. Met de microfluidische chips kunnen we druppels produceren met een straal van ongeveer 250 nm. Met behulp van hogesnelheidscamera's hebben we de initiatie van de druppelverdamping kunnen vastleggen bij verschillende temperaturen, zowel onder als boven het kookpunt van de PFC. Onder het kookpunt bleef het druppel-belcomplex meer dan $500 \mathrm{~ms}$ stabiel doordat de dampbel in de druppel gestabiliseerd kon worden door de druppel na verdamping nogmaals aan te stralen.

Het werk in dit proefschrift beantwoord belangrijke vragen met betrekking tot thermodynamische en akoestische parameters die belangrijk zijn bij de verdamping van druppels met een laag kookpunt. Wij hebben er vertrouwen in dat het gepresenteerde werk de efficintie van de verdamping van druppels met akoestische golven verhoogt met het oog op medische beeldvormende technieken en therapeutische toepassingen. We hebben nieuwe wegen geopend om de benodigde druk voor verdamping van de druppels te verlagen, waarmee automatisch een winst in efficintie werd bereikt. Niettemin blijven er vele open vragen bestaan die beantwoord zullen moeten worden voordat de druppels klinisch toegepast kunnen worden. Het vervolgonderzoek zou zich kunnen richten op de volgende aspecten:

- Het verdampen van druppels in de nabijheid van cellen - het verdampingsproces van druppels met daarin een emulsie van nanodruppels gevuld met een medicijn voor gerichte en gecontroleerde medicijnafgifte wordt tot op heden nog niet volledig begrepen. In de toekomst zou men zich kunnen richten op het kwantificeren van de krachten uitgeoefend op cellen tijdens de medicijnafgifte en het bestuderen van het afgifteproces met hogesnelheidsopnamen. ADV kan naast het afgeven van medicijnen ook zorgen voor een tijdelijke verhoging van de permeabiliteit van het celmembraan, waardoor de opname van medicijnen door de cellen verhoogd kan worden. Hogesnelheidsopnamen van de verdampende druppels dichtbij cellen, waarbij de druppels bovendien gevuld zijn met fluorescerend materiaal, kan de bio-effecten van ADV in kaart brengen en kan tevens inzicht geven in de opname van medicijnen door cellen.

- De fysica van de verdamping van druppels onder het kookpunt - De rol van gas diffusie bij de verdamping van druppels die zich in een omgeving bevinden met een temperatuur lager dan het kookpunt van de drup- 
pels is belangrijk. Theoretische modellen en numerieke simulaties zijn nodig om het principe hiervan beter te kunnen begrijpen.

- Ontwikkeling van transducers die meerdere frequenties kunnen uitzenden - met behulp van een transducer die meerdere ultrageluid frequenties uit kan zenden kunnen de amplitude en fase van het uitgezonden ultrageluid worden aangepast, om de mate van positieve interferentie te maximaliseren, voor een optimale verdamping bij elke gewenste druk.

- Druppelstabilitieit en biocompabiliteit - verschillende recepten voor de microdruppels dienen te worden gevalueerd, om een druppelemulsie te kunnen produceren die niet alleen stabiele bellen produceert, maar waarvan we ook de groei van deze bellen na verdamping kunnen controleren. Hieronder valt onder andere het ontwerpen van druppels bestaande uit meerdere vloeistoffen met verschillende akoestische eigenschappen, om zo bijv. de akoestische impedantie te variren via de dichtheid en de geluidssnelheid, teneinde het focusserende effect te maximaliseren. Om toekomstig klinisch gebruik van deze technieken mogelijk te maken, is onderzoek naar de biocompatibiliteit van de druppelemulsies van groot belang.

Het gebruik van PFC druppels als contrastmiddel voor ultrageluid is vrij nieuw, en heeft mogelijkerwijs voordelen ten opzichte van de bestaande contrastmiddelen. In dit proefschrift hebben wij de fysische aspecten van het verdampen van druppels beschreven, die nodig zijn voor het om ze succesvol toe te passen voor beeldvormende technieken en voor therapeutische doeleinden. Wij geloven daarom dat deze innovatieve technieken en nieuwe inzichten een potentieel nieuw wapen kunnen vormen in de strijd tegen kanker door een betere detectie en uiteindelijk een betere behandeling met behulp van ultrageluid. 


\section{Scientific output}

\section{Publications}

\section{Book chapters}

1. Oleksandr Shpak, Martin Verweij, Nico de Jong, and Michel Versluis, "Droplets, Bubble \& Ultrasound interaction", "Therapeutic Ultrasound" book to Springer's Editions, Editor: Jean-Michel Escoffre. Submitted on 19th of July 2014 as a book chapter.

\section{Peer-reviewed journals}

2. Nikita Reznika, Oleksandr Shpak, Erik C. Gelderblom, Ross Williams, Nico de Jong, Michel Versluis, and Peter N. Burns, ”The efficiency and stability of bubble formation by acoustic vaporization of submicron perfluorocarbon droplets”, Ultrasonics 53 (7). 1368-1376 (2013).

3. Oleksandr Shpak, Martin Verweij, Rik Vos, Nico de Jong, Detlef Lohse, and Michel Versluis, "Acoustic droplet vaporization is initiated by superharmonic focusing", Proceedings of the National Academy of Sciences of the United States of America 111 (5). 1697-1702 (2014).

4. Oleksandr Shpak, Tom Kokhuis, Ying Luan, Detlef Lohse, Nico de Jong, Brian Fowlkes, Mario Fabiilli, and Michel Versluis, "Ultrafast dynamics of the acoustic vaporization of phase-change microdroplets", Journal of the Acoustical Society of America 134 (2). 1610-1621 (2013).

5. Oleksandr Shpak, Laura Stricker, Michel Versluis, and Detlef Lohse, "The role of gas in ultrasonically driven vapor bubble growth", Physics in Medicine and Biology 58 (8). 2523-2535 (2013). 
6. Nikita Reznik, Guillaume Lajoinie, Oleksandr Shpak, Erik C. Gelderblom, Ross Williams, Nico de Jong, Michel Versluis, and Peter N. Burns, "On the acoustic properties of vaporized submicron perfluorocarbon droplets", Ultrasound in Medicine \& Biology 40 (6). 1379-1384 (2014).

7. Oleksandr Shpak, Tim Segers, Lingling Shui, Guillaume Lajoinie, Jan Eijkel, Nico de Jong, Detlef Lohse, and Michel Versluis, "Monodisperse droplets for ADV". To be submitted. 


\section{Acknowledgements}

First of all I'm grateful to Michel Versluis, Detlef Lohse and Nico de Jong for giving me the opportunity to work in the group. Thank you for having faith in my abilities and inspiring me for greater achievements. Thank you for all the support and the encouragements regarding the $\mathrm{PhD}$ research.

In particular, I would like to thank to my supervisor, Michel Versluis. I would have never been able to achieve my scientific results without his excellent guidance. Michel has a very good feeling of subjects which would attract a big attention from the scientific community. I always had many projects and ideas in my head, however Michel pointed out the best of them, and once being completed, drew substantial recognition in the scientific community. Every time I proclaimed that I explained observed phenomena, each time Michel was overcritical, suggesting and thinking about other tests and parameters that would otherwise prove my claims. This greatly helped to communicate with publication referees, as our arguments were always very convincing. Michel is a very good teacher in terms that he can explain complicated things in a very efficient and easy way. Michel also has a very strong vision regarding future directions and projects, so each time we had a meeting, listening to what I was telling him, he could come up with much better prospective of where my idea can be applied or he could even see the entirely new research directions for future research proposals. Besides scientific matters, Michel greatly helped with my oral presentation skills and English writing. He spent enormous amount of time on giving me feedback about articles and presentations structure, statements, formulas and figures, helping to make them into an easy to understand, and pleasant to read form.

I would like to thank Nico de Jong. Nico helped to organize a couple of very costly and difficult projects with scientific groups overseas. Nico gave me his enthusiasm and support for ideas no matter how difficult they were in terms of both human and equipment resources. To give examples, not so far after starting my $\mathrm{PhD}$ project Nico sent me to an international conference 
in the USA, so that I could absorb knowledge and learn what clinical people are doing, even though at that time I had not a single result to show. Nico always supported me with additional experiments and by arranging them in the Rotterdam labs. I very much appreciate such support, Nico, because it allowed me to keep up with the fast pace and made my scientific output within the allocated time.

I would also like to thank Detlef Lohse for sharing his experience and being a source of inspiration. I'm very grateful for our fruitful brainstorming discussions, were Detlef could insightfully point out all the crucial features, so that it could save me an enormous amount of time discovering it on my own. An overwhelming number of ideas, implemented in this thesis, were born during such meetings, where Detlef, Michel and Andrea were discussing together with me what I was showing to them. I very much appreciated that Detlef immediately reacted and substantially helped when I said that I was looking for a future job position. Detlef is aware and remembers what happens outside the scientific life of people in his group and is always willing to help.

I would like to thank Martin Verweij. Martin spent an enormous amount of energy on our joint project on acoustic droplet vaporization focusing effect. He developed the tools to overcome computational limitations and he was very responsive to all my questions, no matter when I asked them, be it on weekends or after midnight. Martin was very careful with every little detail and was always giving me very useful feedback on how I should formulate my statements. Thank you for agreeing to be on my $\mathrm{PhD}$ defense committee.

I would like to thank Andrea Prosperetti for his experience and wisdom. I have benefited tremendously from interactions with him. After following his suggestions I could immediately solve problems on which I was sometimes struggling for almost a year.

Many things went smooth due to the support of Joanita Leferink, GertWim Bruggert, Martin Bos and Bas Benschop. Thank you for your help and for solving all the tasks with great kindness, efficiently and in a pleasant and easy way.

A substantial part of the scientific support was thanks to my collaborators and coauthors: Erik Gelderblom, Laura Stricker, Tim Segers, Guillaume Lajoinie, Nikita Reznik, Lingling Shui, Jan Eijkel, Claudia Errico, Olivier Couture, Tom Kokhuis, Ying Luan, Mario Fabiilli, Brian Fowlkes, Michelle Adan and Oliver Kripfgans.

I've enjoyed the company of my colleagues Vivek, Siddartha, Federico, Minori, Michiel, Ilya, Pascal, Bram, Wilco, Wim, Maarten, Sander H., Mark- 
Jan, Rodolfo, Leen, Jacco, Devaraj, Chao, Martin, Oscar, Erik-Jan, Sander W., Koen, Joost, Claas-Willem, Tuan, Daniela, Yoshi, Tak, Ceyda, Enrique, Ivo, Marlies, Julian, Erik, Rianne, Henri, Rajaram, Stefan, Dennis ,Theo, Antonin, Tess and Alvaro.

A very special thanks to my parents. I'm happy that they will travel all the way to be here with me at my $\mathrm{PhD}$ defense. Finally, I thank my wife Kseniia, you are my greatest support.

This work is part of the research program of the Foundation for Fundamental Research on Matter (FOM), which is part of the Netherlands Organization for Scientific Research (NWO). 


\section{About the author}

Oleksandr Shpak was born on 26th of June, 1987 in Vinnycya, Ukraine. He graduated from the Ukrainian Lyceum of Physics and Mathematics in 2004. Soon after his graduation he was awarded the Gold Medal in the 35th International Physics Olympiad in Pohang, South Korea.

In 2004, for his undergraduate degree, Oleksandr enrolled in Taras Shevchenko National University of Kyiv to study physics.

In 2008, he was awarded a scholarship for the Top Master Program at the University of Groningen and moved to the Netherlands. He obtained his Master degree in Nanoscience with the subject Switching Casimir Forces with Phase Change Materials at the Nanostructured Materials and Interfaces research group under the supervision of Prof. George Palasantzas.

In 2010, Oleksandr joined the Physics of Fluids Group at the University of Twente as a PhD candidate under the guidance of Prof. Michel Versluis, Prof. Nico de Jong and Prof. Detlef Lohse to study acoustic droplet vaporization, the results of which are presented in this thesis.

Oleksandr will continue his career at ASML in Veldhoven, the Netherlands as a Performance Analyst. ASML is currently the largest supplier in the world of photolithography systems for the semiconductor industry. 